\title{
Cutaneous Leishmaniasis in British Troops from Belize.
}

Neill Christopher Hepburn

Submitted for the degree of

\author{
Doctor of Medicine \\ of the \\ University of Edinburgh \\ 1994
}

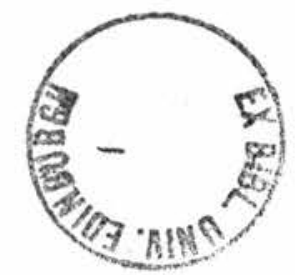




\section{Contents}

Page

Declaration

4

Abstract

5

Publications and Presentations

6

Acknowlegements

8

Chapter 1: Introduction and literature review.

Aims of the study

General review of cutaneous leishmaniasis

Cutaneous leishmaniasis and the British army

garrison in Belize

Chapter 2: A retrospective study of Belizian cutaneous

leishmaniasis in British troops from 1978 to mid-1990.

Chapter 3: Hepatotoxicity of sodium stibogluconate therapy.

Chapter 4: Cardiotoxicity of sodium stibogluconate therapy.

Chapter 5: Aminosidine (paromomycin) versus sodium leishmaniasis from Belize.

Chapter 6: A study of the diagnostic pathology.

Chapter 7: Conclusions and management guidelines.

References

Appendices:1. Data for chapter 2. 
2. Data for chapter 3.

179

3. Data for chapter 4.

182

4. Consent form and data for chapter $5 \quad 211$

5. Publications 


\section{Declaration}

I declare that I have written this thesis myself. The studies described represent my own ideas and approach to improving the clinical management of soldiers who contract cutaneous leishmaniasis in Belize. I obtained permission and funding from the Ministry of Defence to study this problem in Edinburgh. I designed and performed the studies, analysed the results, and prepared them for publication in this thesis and as papers.

I received technical advice and assistance with several of the studies. Dr $P$ Hayes, of the Department of Medicine, and Dr AF Howie, of the Department of Clinical Biochemistry, assisted me with the technical aspects of the hepatotoxicity study. Professor KAA Fox, and Dr J Nolan of the Cardiovascular Research Unit and Dr JMM Neilson, of the Department of Medical Physics and Medical Engineering assisted me with the technical aspects of the cardiotoxicity study. Dr. L Biddlestone assisted me with the pathological study. Dr D Evans performed the cultures and typing of the leishmania.

I started the retrospective case-note survey described in chapter 2 whilst working at the Cambridge Military Hospital. However most of that study and all the other studies have been undertaken during my appointment as an Honorary Senior Registrar in the University Department of Dermatology in The Royal Infirmary of Edinburgh from May 1991 until March 1994. 


\section{Papers and Presentations}

As a result of this study several papers, reviews and presentations have been prepared.

\section{Papers}

Hepburn NC, Tidman MJ, Hunter JAA (1993): Cutaneous leishmaniasis in British troops from Belize.

British Journal of Dermatology $128 ; 63-68$.

Hepburn NC, Siddique I, Howie AF, Beckett GJ, Hayes PC (1993):

Hepatotoxicity of sodium stibogluconate in leishmaniasis.

Lancet 342; 238-239 (letter).

Hepburn NC, Siddique I, Howie AF, Beckett GJ, Hayes PC (1994):

Hepatotoxicity of sodium stibogluconate therapy in American

cutaneous leishmaniasis.

Transactions of the Royal Society of Tropical Medicine and Hygiene (in press).

Hepburn NC (1993): Thrombocytopenia complicating sodium stibogluconate therapy for cutaneous leishmaniasis.

Transactions of the Royal Society of Tropical Medicine and Hygiene 87; 691.

Biddlestone LR, Hepburn NC, McLaren KM: A clinico-pathological study of American cutaneous leishmaniasis.

Transactions of the Royal Society of Tropical Medicine and Hygiene (in press).

Hepburn NC, Tidman MJ, Hunter JAA: Aminosidine versus sodium stibogluconate therapy for cutaneous leishmaniasis from Belize.

Transactions of the Royal Society of Tropical Medicine and Hygiene (in press).

Hepburn NC, Nolan J, Fenn L, Herd RM, Neilsen JMN, Sutherland GR, Fox KAA: Cardiac effects of sodium stibogluconate: an electrocardiographic and echocardiographic study. (under submission).

\section{Reviews}

Hepburn NC (1992): The management of American cutaneous leishmaniasis. Journal of Dermatological Treatment 3; 137-138.

Hepburn NC (1993) Cutaneous leishmaniasis. Part i, clinical features. Proceedings of the Royal College of Physicians of Edinburgh 22; 6368. 
Hepburn NC (1993) Cutaneous leishmaniasis. Part ii, historical aspects, epidemiology and prevention.

Proceedings of the Royal College of Physicians of Edinburgh 22; 140-150.

\section{Papers presented at meetings}

Hepburn NC, Tidman MJ, Hunter JAA: Cutaneous leishmanisis in British Troops from Belize.

18th World Congress of Dermatology, New York, June 1992.

Hepburn NC, Tidman MJ, Hunter JAA: An open randomised prospective study of aminosidine versus sodium stibogluconate in the treatment of American cutaneous leishmaniasis - preliminary results.

Dermatology 2000, Vienna, May 1993.

Hepburn NC, Tidman MJ, Hunter JAA: Aminosidine (paromomycin) versus sodium stibogluconate in American cutaneous leishmaniasis.

British Association of Dermatologists, Oxford, July 1993. 


\section{Acknowledgements}

My interest in cutaneous leishmaniasis began in 1987 when, as an $\mathrm{SHO}$ in dermatology, I treated many soldiers who has contracted the disease whilst jungle training in Belize. This interest was encouraged by my supervising consultants, Colonel D Sim-Davis and Lt Col K Freeman, and also by Colonel DS Jolliffe.

Dr ADM Bryceson and Dr D Evans kindled my enthusiasm and have given me invaluable advice from time to time.

I was further encouraged to continue my investigations by Professor JAA Hunter and Dr MJ Tidman when I arrived to work in the University Department of Dermatology in Edinburgh. They gave me patient and pertinent advice on many aspects of medical research ranging from study design to the analysis and presentation of results.

I was fortunate to have had the support of Brigadier GO Cowan who, as the Professor of Military Medicine, sponsored my investigation into the efficacy of aminosidine and helped me obtain the necessary approval from the Ministry of Defence. I am indebted to the Commanders Medical in Scotland, Colonel WSP McGregor and Lt Col M Staunton, who allowed me to treat soldiers in the Medical Reception Station in Edinburgh. I am grateful to Dr M Paterson who allocated me several of his beds. I am particularly grateful to the Matron, Major J Thornton, for her unflagging support and encouragement throughout all the difficulties we encountered. The army nurses and medical assistants not only took an interest in the study but also great pride in ensuring that everything went according to plan.

I have been fortunate to collaborate with other departments within the Royal Infirmary of Edinburgh during several of the studies. They have given me helpful advice and instruction in their specialist areas without which those studies would have been impossible. They are Professor KAA Fox, Dr J Nolan, Dr G Sutherland and Mrs L Fenn of the Cardiovascular research Unit; Dr P Hayes, Dr I Siddique of the Department of Medicine, DR F Howie and Dr 
G Beckett of the Department of Clinical Biochemistry; Dr L Biddlestone and Dr K M McLaren of the Department of Pathology.

Throughout my time in Edinburgh I have received superb administrative and secretarial assistance from Mrs M Gray and Miss $\mathrm{J}$ Liston; without their help writing the papers and this thesis would have been much more difficult.

Finally I must thank my wife, Julia, and my children, Amy and William, whose time it took to perform these studies and write this thesis. This has been a selfish activity on my part and their forbearance has been remarkable. 


\section{Abbreviations}

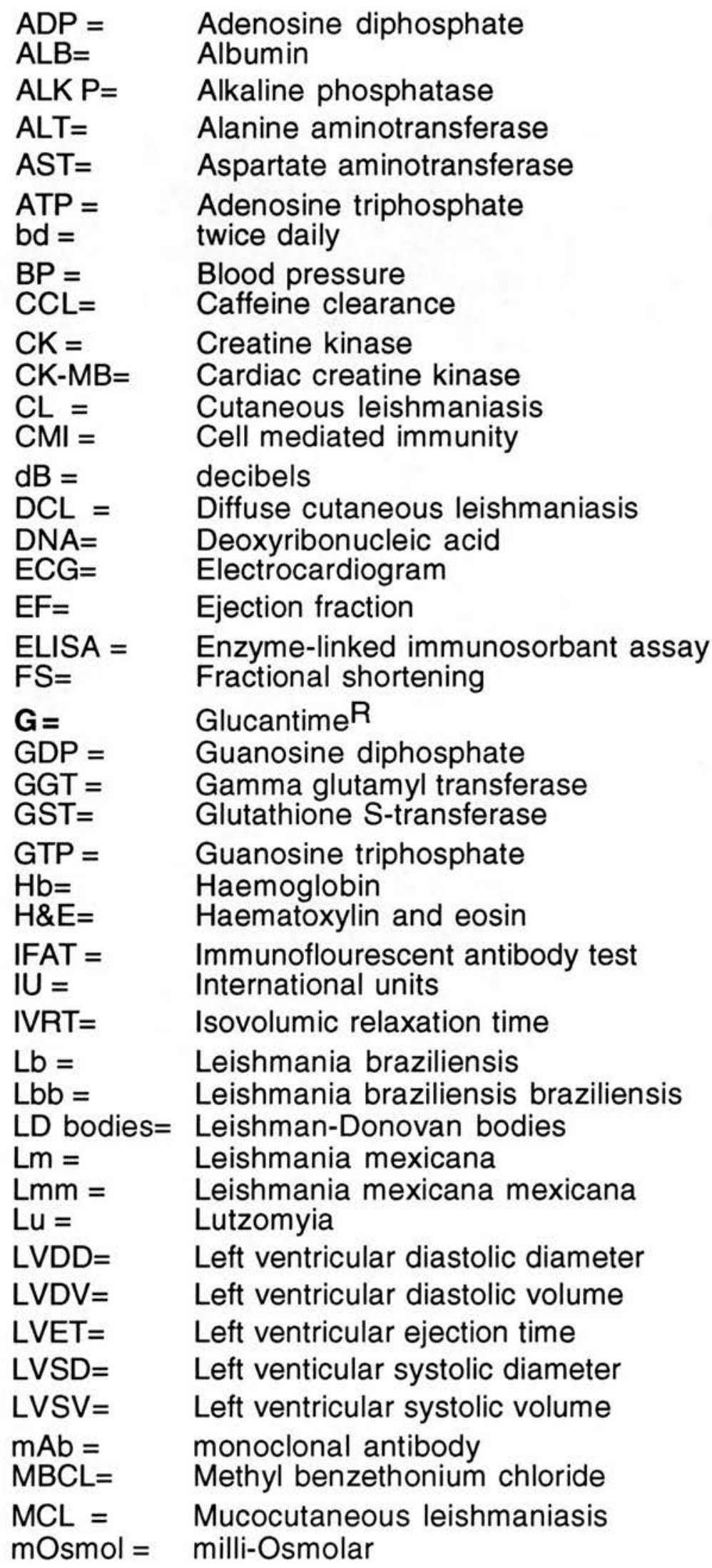




$\begin{array}{ll}n= & \text { Number } \\ \text { NA }= & \text { Not available } \\ \text { NS }= & \text { Not stated } \\ \text { od }= & \text { once daily } \\ \mathrm{P}= & \text { Phlebotomus } \\ \mathrm{p}= & \text { Probability of no difference } \\ \mathrm{P}= & \text { Pentostam } \mathrm{R} \\ \mathrm{PEP}= & \text { Pre-ejection period } \\ \mathrm{PKDL}= & \text { Post Kala-azar dermal leishmaniasis } \\ \mathrm{PT}= & \text { Prothrombin time } \\ \mathrm{sd}= & \text { standard deviation } \\ \mathrm{se}= & \text { standard error of the mean } \\ \mathrm{UK}= & \text { United Kingdom } \\ \text { USA }= & \text { United States of America } \\ \text { VL }= & \text { Visceral leishmaniasis } \\ \text { WHO }= & \text { World Health Organisation }\end{array}$




\section{Chapter 1}

Introduction and literature review

1.1 Aims of the study

1.2 General review of cutaneous leishmaniasis

1.2.1 Historical overview.

1.2.2 Epidemiology.

1.2.3 Pathology, pathogenesis and immunology.

1.2.4 Clinical features.

1.2.5 Diagnosis.

1.2.6 Treatment.

1.3 Cutaneous leishmaniasis and the British army garrison in Belize

1.3.1 The history and rôle of the British garrison.

1.3.2 The cutaneous leishmaniasis problem. 


\subsection{Aims of the study}

My overall aim in the various components of this study has been to improve the management of cutaneous leishmaniasis contracted by British troops during operational tours of duty in Belize. I started this study in March 1990 whilst working in the Dermatology Department of the Cambridge Military Hospital in Aldershot. It was interrupted for six months whilst I was on active service in Saudi Arabia during the Gulf War, but restarted when I came to work in the University Department of Dermatology in Edinburgh. Inevitably knowledge of cutaneous leishmaniasis has increased, facilities have changed and my own understanding of the problem has become greater as the study has progressed. I have therefore explored aspects of the problem which I had not originally considered. However, my aims during this study can be summarised as follows:

To determine the clinical features, types and response to therapy of cutaneous leishmaniasis from Belize.

To assess the hepatotoxicity of sodium stibogluconate in the treatment of cutaneous leishmaniasis.

To assess the cardiotoxicity of sodium stibogluconate in the treatment of cutaneous leishmaniasis.

To compare the efficacy and toxicity of aminosidine (paromomycin) with sodium stibogluconate for treatment of cutaneous leishmaniasis from Belize.

To evaluate the diagnostic pathology. 


\subsection{General Review of Cutaneous Leishmaniasis}

\subsubsection{Historical Overview}

The leishmaniases are ancient afflictions of man. In the Old World, descriptions of Balk Sore (named after Balk a town in the north of Afghanistan near the Russian border) date back to the first century AD. The disease has been traced back through the mediaeval literature of the Middle East by Elgood (1934) who speculated that it was taken from Balk to Bagdad with the invading Mongols in $1215 \mathrm{AD}$ and that it subsequently spread down to the Persian Gulf and, later, back along the trade routes into the interior of Iran reaching Tehran in 1840. The disease has many synonyms, mostly derived from place names such as Delhi boil, Aleppo boil, Armenian sore, Sart sore and the still commonly used Oriental sore (Gardiner 1977).

In the New World early records of cutaneous leishmaniasis (CL) are more difficult to identify because other endemic conditions, such as syphilis and blastomycosis, cause similar lesions. However mucocutaneous leishmaniasis (espundia) can be traced back to pre-Columban times through anthropomorphic vessels or 'huacos' which have been found amongst the ancient Peruvian mochica pottery obtained from Inca tombs (circa $400-900$ AD). Some of these figures depict the typical facial lesions, as do terracotta figures from Northern Equador. Shortly after the Spanish conquest historians, such as Pizarro, noted a rare, but decimating, disease amongst the Peruvian Indians which primarily affected the nasal cavities and occurred in the same locations as espundia today:

'Indians are taken from the highlands to work in the coca fields in the 'Andes of Cuzco' where they get an infection in the nostrils which also makes their nose trunk-like and red, from which some die.'

'.......and the Indians get sick with an incurable disease called 'of the Andes' which is worse than syphilis......it attacks most commonly the nostrils, lips and uvula of the throat... ..others get it in the mouth, making it a sight one cannot look at without great horror and fright at such ugliness.' 
In addition, Spaniards who explored the high Andean valleys, where sandflies were common, occasionally developed mutilating lesions of the nares similar to those depicted on the huacos. The disease was known as 'anti-onccoy' or 'the disease of the Andes' but only comparatively recently has the name of the long suspected cause - a small biting fly or 'uta' - been applied to the disease itself (Gardiner 1977). Names for the disease in its various forms are also profuse in the New World and include uta, espundia, mucocutaneous leishmaniasis, ulcera des los chicleros, boton de Baura, bouba, bay sore, forest yaws, pain bois and leishmaniose tegumentar americana.

The scientific study of leishmaniasis began with the publication in 1885 in Scientific Memoirs by Medical Officers of the Army of India by Surgeon-Major D.D.Cunningham (1843 - 1914) of a description of amastigotes in sections of human skin from a patient with Dehli boil. He described some 'deeply staining bodies in certain cells larger than lymphocytes' and attributed them to the spores of a slime mould. These observations were confirmed six years later by Firth (1891).

In 1898 Borovsky, a Russian military surgeon studying Sart sore in Tashkent, described 'small spherical, oval or fungal-form corpuscles' measuring 1.5-2.0 um diameter with a nucleus and a 'process running to the periphery'. Unlike Cunningham, he realised they were protozoa and the cause of the disease. Unfortunately his observations were published in an obscure Russian journal 'Voenno-Medicinskij-Zürnal' and the significance of his discovery was not recognised for many years (Gardiner 1977).

In 1903 the pace of discovery quickened, Leishman (1865-1926) a British army pathologist (figure 1.1), reported the similarity of degenerating trypanosomes from the spleen of a laboratory rat, which had died after an experimental infection with African trypanosomes 48 hours earlier, and bodies he had observed 3 years previously during a post-mortem examination of the splenic pulp of a soldier 36 hours after death from a tropical cachexia. The soldier had contracted this illness during service at Dum-Dum 7 miles from Calcutta (Leishman 1903). Leishman concluded that these organisms had been degenerating trypanosomes which, although incorrect, this was the first 
Figure 1.1: Lieutenant-General Sir William Boog Leishman (1865-1926).

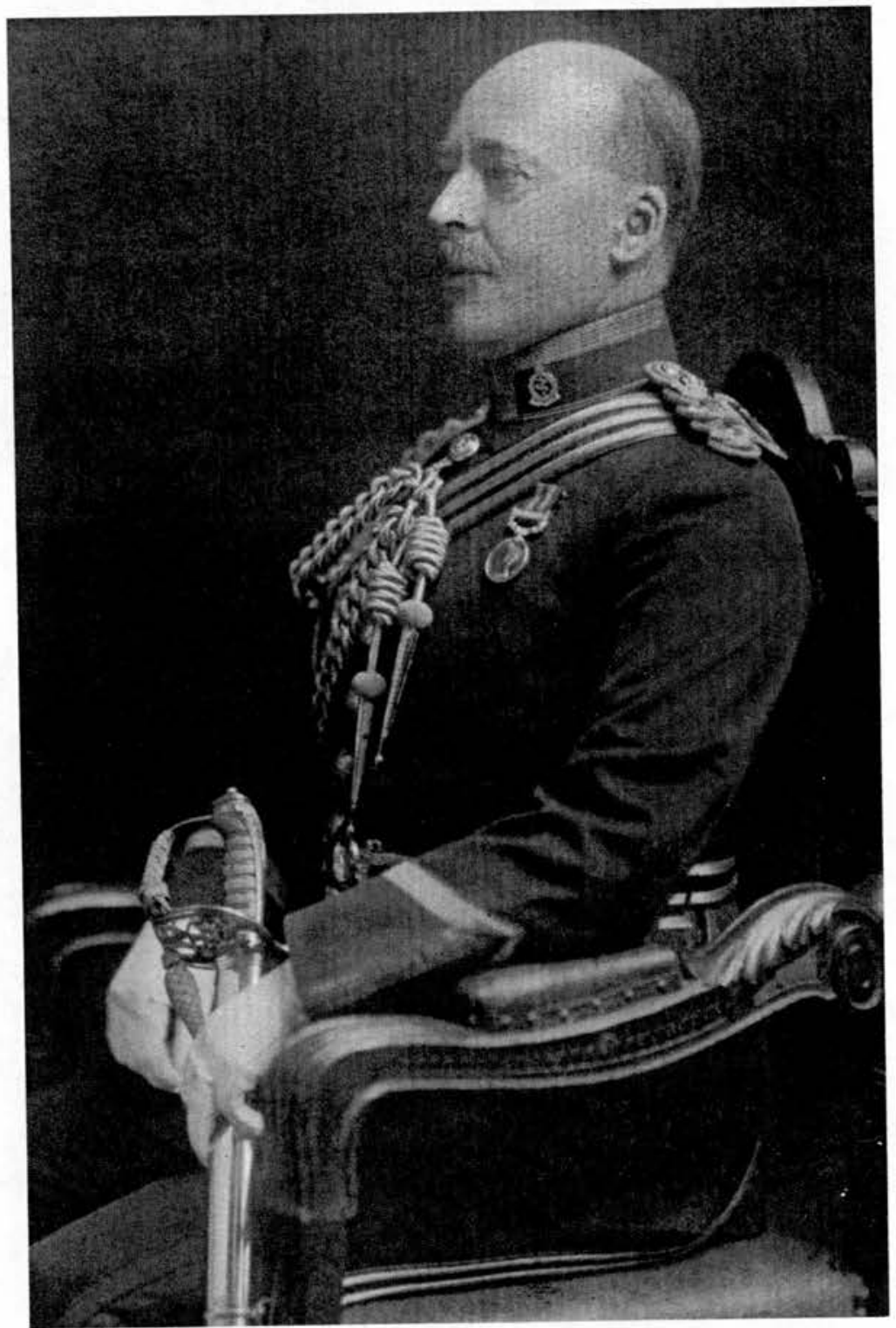

description of the aetiological agent of visceral leishmaniasis. Subsequently Donovan, a Captain in the Indian Medical Service at Madras, described similar organisms in a fresh splenic biopsy thereby demonstrating it was a distinct organism rather than a degenerate trypanosome (Donovan 1903). Later the same year Wright, whilst working in Boston, USA, and using a greatly improved Romanowsky stain, first accurately described and photographed the cutaneous Old World parasite which he obtained from the cheek of a nine year old girl who had developed the lesion 2 or 3 months earlier whilst in Armenia (Wright 1903). 
In 1904 Rogers successfully cultured the visceral parasite by incubating cultures at $22^{\circ} \mathrm{C}$ and in 1908 Nicolle cultured the cutaneous organism using a modification of the rabbit blood nutrient agar medium of Novy and McNeal known as NNN medium (Gardiner 1977).

The first report of the New World organism was made by Lindenburg in 1909. $\mathrm{He}$ had been examining ulcers on the face and forearms of railway workers in Sao Paulo. Two years later, the clinical features of mucocutaneous leishmaniasis (espundia) were described by Carini whilst working at the Pasteur Institute in Sao Paulo. In the same year Vianna described parasites he had observed in ulcers contracted in Minas Gerias, Brazil - he was able to discern a nucleus, kinetoplast and a 'rudimentary flagellum' and proposed the name Leishmania braziliensis to distinguish it as the cause of espundia (Vianna 1911). In 1913 an expedition from Harvard investigated and succeeded in discovering, independently, the aetiology of 'uta' in Peruvian mountain towns. They described and illustrated the parasites together with the clinical features including erosion of the palate. They were also able to culture the organism on blood agar and believed that it was transmitted by the genus Phlebotomus (Gardiner 1977).

The sandfly has been implicated as the vector of cutaneous leishmaniasis as far back as 1764 (Herrer and Christensen 1975). However the cycle of infection was not worked out until this century. In 1904 Rogers observed the conversion of amastigotes to promastigotes in culture (Rogers 1904) and then promastigotes were found in sandflies (Alder and Theodor 1925). In 1921 a volunteer developed a cutaneous lesion following inoculation with a suspension of caught phlebotomus (Sergent et al 1921) but not until 1941 was L.tropica transmitted to volunteers by the bite of experimentally infected Phlebotomus papatasii thus confirming the cycle (Alder and Ber 1941).

The taxonomy of leishmania has been, and remains, a vexed subject. Early workers assumed that leishmania in geographically widely separated foci, causing different patterns of disease, were due to different species and gave them different names. It then became possible to separate them on the basis of their morphology and differences in their behaviour in the natural sandfly host, the vertebral host and in vitro culture media. In recent years more 
precise identification has become possible through isoenzyme electrophoresis and DNA analysis. In 1961 Pessoa thought that all forms of visceral leishmaniasis were due to a single species, L.donovani. He applied the name L.tropica to the parasite of the Old World and gave leishmania of the New World the names L.braziliensis braziliensis and L.braziliensis mexicana. In the 1970's Lainson and Shaw divided the parasites of the New World into two main groups, the L.mexicana complex and L.braziliensis complex on the basis of their developmental patterns in sandfly vectors, laboratory hamsters, blood agar culture media and buoyant density values for nuclear and kinetoplast DNA. Then, in 1974, Lumsden combined the L.mexicana complex and L.braziliensis complex groups of Lainson and Shaw for the New World organisms with those of the L.tropica complex proposed by Pessoa for the Old World and placed parasites normally associated with human visceral leishmaniasis into an L.donovani complex (Gardiner 1977).

Leishmaniasis was first successfully treated in 1912 by Vianna in Brazil who used the toxic antimony compound potassium antimonyl tartrate ('tartar emetic') for cases of CL and mucocutaneous leishmaniasis (MCL) (Vianna 1912, Vianna 1914). The medicinal use of antimony dates back to the teachings of Paracelsus in the early 16th century who observed that white wine, which was allowed to stand in antimony goblets, acquired emetic properties because the tartaric acid in the wine interacted with the metal to form tartar emetic. Although popular for a time it was subsequently abandoned because of its toxicity and lack of specific medicinal properties. The discovery of its anti-parasitic properties, initially against trypanosomiasis, led to its reintroduction during the early part of this century. It was mainly used for kala-azar but its efficacy remained controversial and its toxicity a major problem as described by Cole:

'Tartar emetic is not a pleasant drug; it produces cough, chest pain, and great depression, just after injection, so that patients have often refused to continue with it.......it is a definite poison with action on the heart, for one case died of sudden heart failure within an hour of injection, and others appeared to be hastened towards heart failure by it'

after 12 of 27 patients treated for kala-azar died he concluded that:

(tartar emetic was) 'not much better than....no treatment at all'.

(Cole 1944) 
Although some aspects of toxicity could be overcome by administering it intravenously the search for better tolerated derivatives continued and in the 1920 's the first pentavalent antimonials, such as urea stibamine and stibosan, became available. Sodium stibogluconate (Pentostam) was first manufactured and used between 1935 and 1945 (Berman, 1988). Since that time many other pharmacological and physical treatments for cutaneous leishmaniasis have been proposed but pentavalent antimonials remain the mainstay of therapy for New World cutaneous and visceral disease.

\section{2..2 Epidemiology}

It has become apparent during the past decade that the leishmaniases - both visceral and cutaneous - are much more prevalent than had been appreciated. In 1990 the World Health Organisation (WHO) estimated that 12 million people were affected in over 80 countries and that 350 million people were at risk of infection (WHO 1990). More specifically it has been estimated that approximately 300,000 cases of cutaneous disease occur each year in 61 countries and that 185 million people are at risk; of those 59,300 occur in the Americas and the population at risk there is 39 million (Ashford et al 1992). The true incidence of cutaneous leishmaniasis tends to be substantially underestimated because of inadequate reporting (Copeland et al 1990), but there is good evidence for a dramatic increase in the incidence of cases in the New World (Vieira et al 1990). The geographical distribution of cutaneous leishmaniasis is shown in figure 1.1 and the epidemiology is summarised in table 1.1. It is important to appreciate, however, that more than half the cases occur in just 8 countries: Afghanistan, Algeria, Brazil, Iran, Iraq, Saudi Arabia, Sudan and Syria (Ashford et al 1992).

The cycle of infection The leishmaniases are complex protozoal infections transmitted by the bite of an infected female sandfly. In most cases these infections exist as zoonoses amongst wild animals and man is only infected as an incidental host (figure 1.2). However such infections are by no means uncommon - in endemic areas up to $9 \%$ of the healthy population may have a positive leishmanin skin test indicative of an earlier, often asymptomatic, 
Figure 1.1: Geographic distribution of CL.

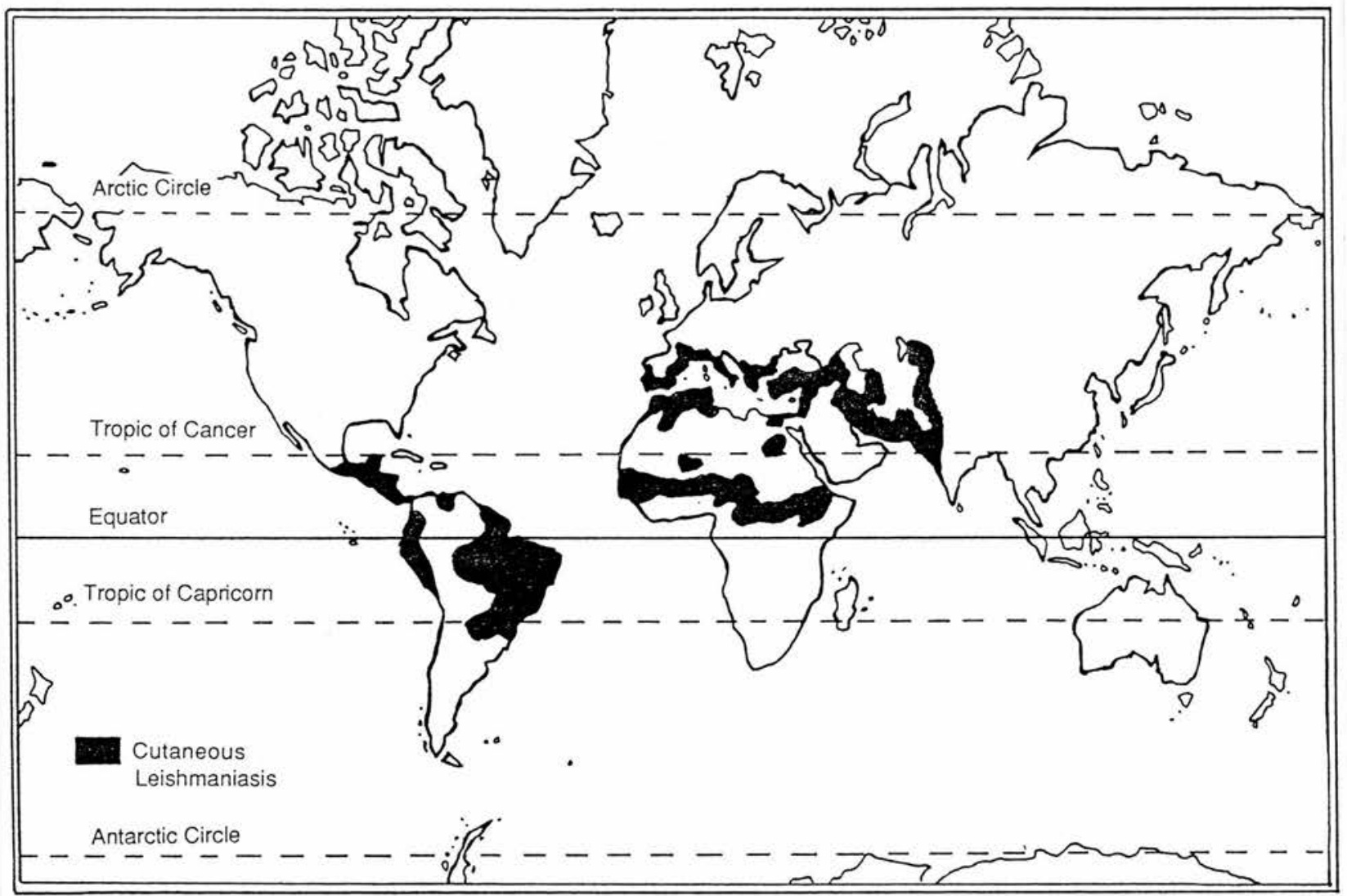

Figure 1.2: The cycle of infection.

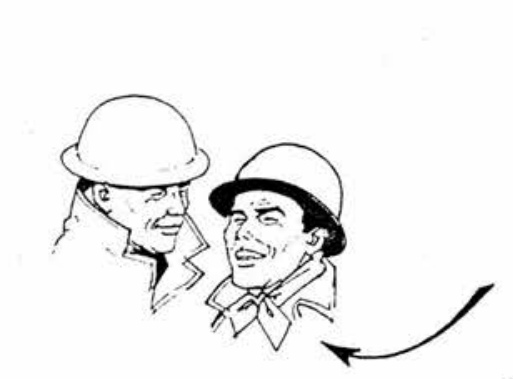

Man (usually accidental host)

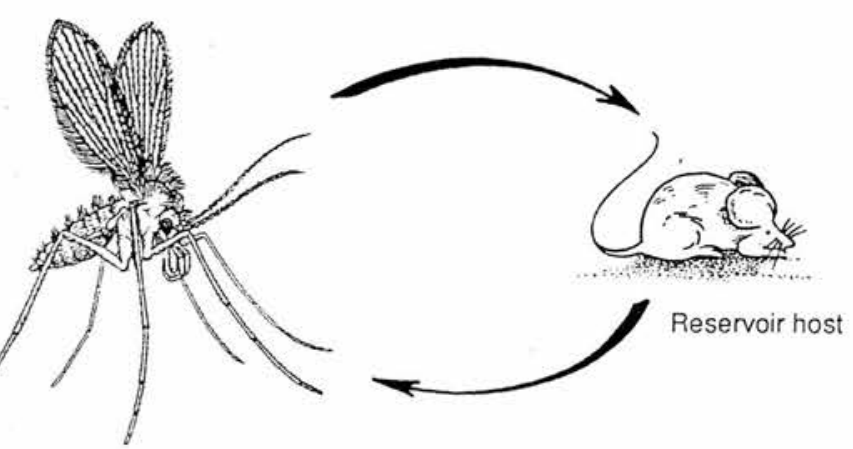

O Sandfly vector 


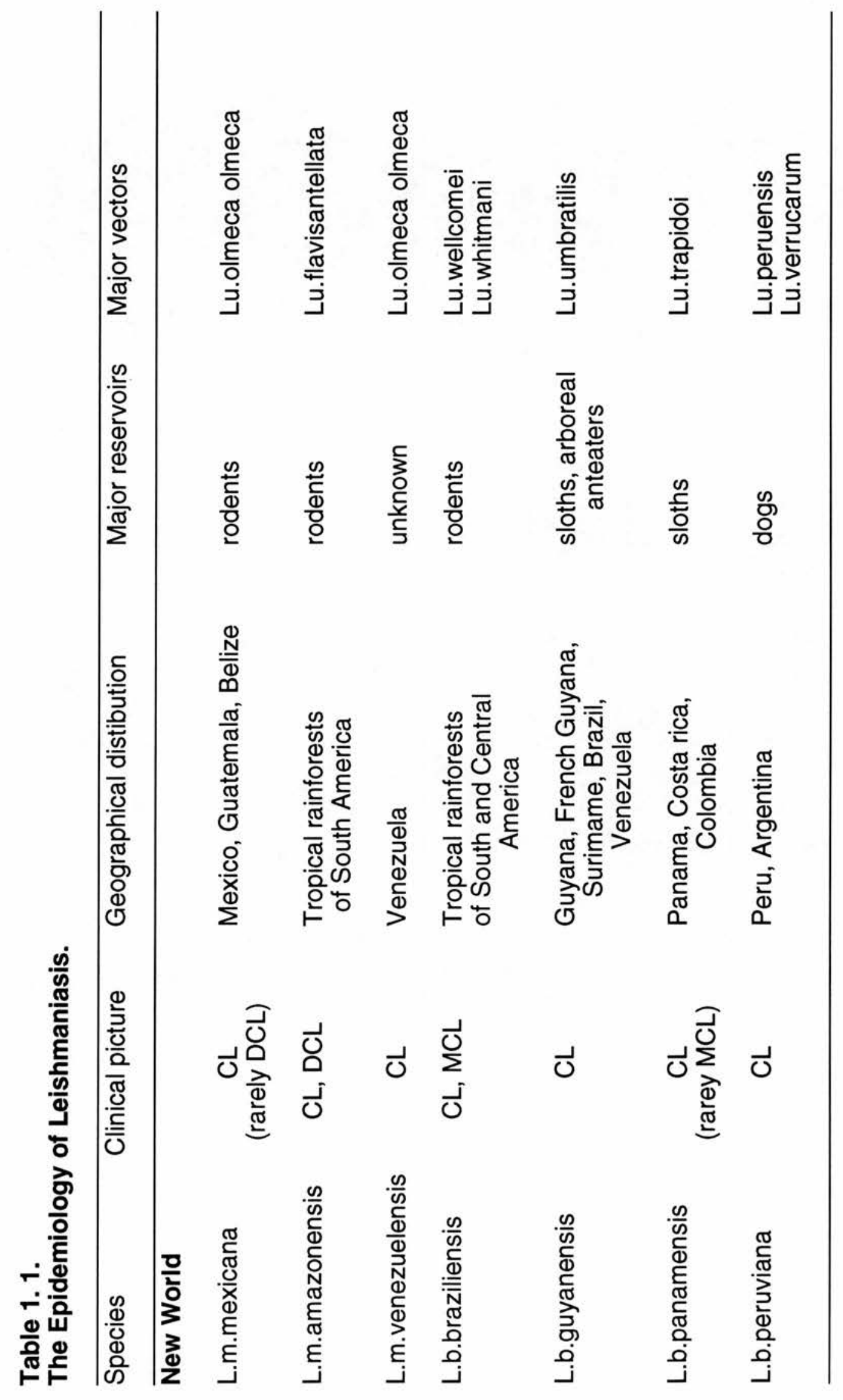




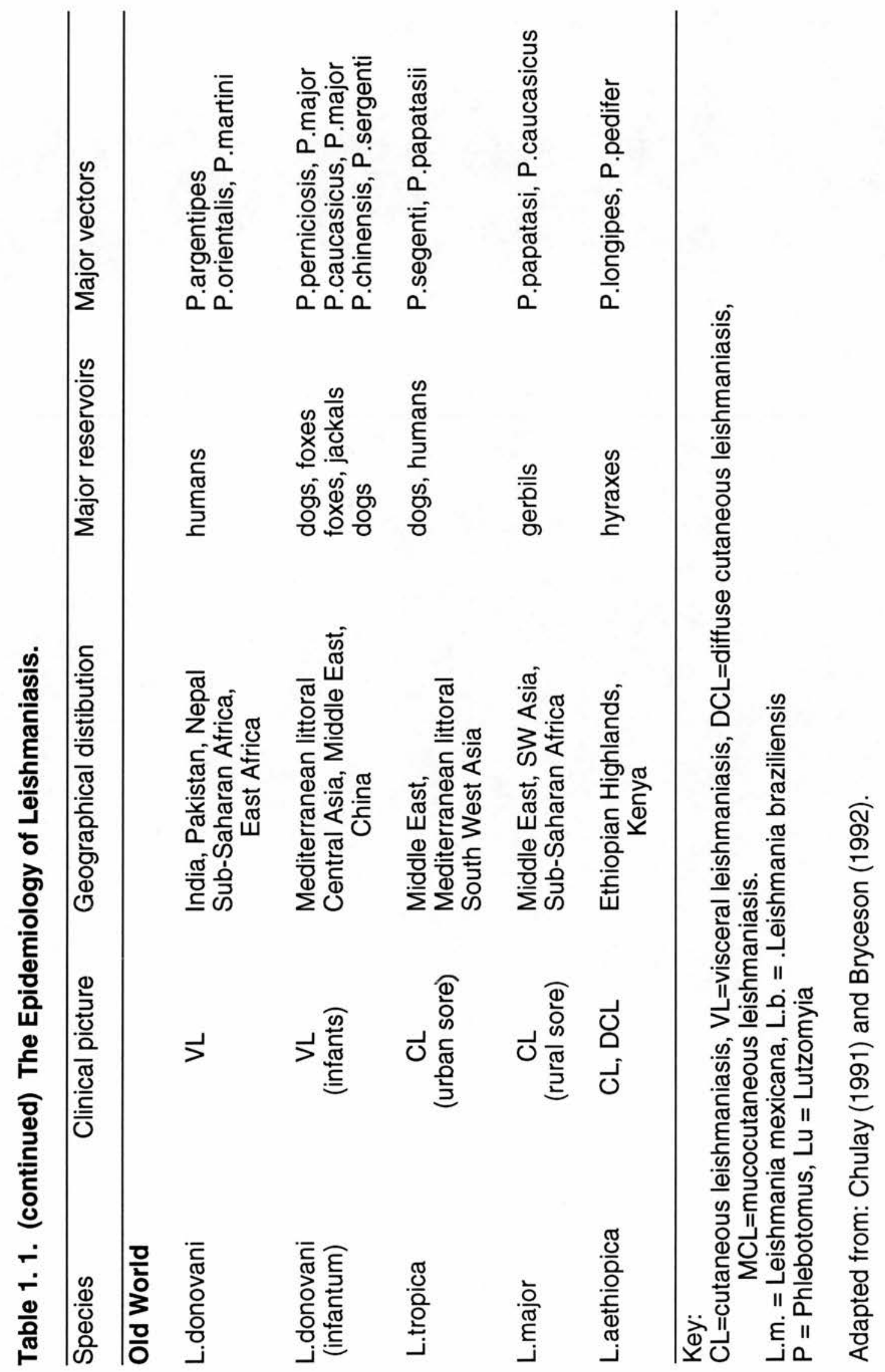


infection (Weigle et al 1991). The vector species, reservoir hosts and environmental requirements tend to be very specific for each leishmanial species and consequently the disease is often localised to small areas ('hot spots') where all three factors exist together.

Vector Biology. Sandflies are extremely small, measuring less than $3.5 \mathrm{~mm}$, they are covered with a sandy yellow or brown coat of hair and have very dark eyes. The male has prominent terminal organs for grasping the female. They live in dark, damp places and make characteristic little hops when disturbed. They are able to fly reasonably well, and although their usual radius is only 50 metres from their breeding sites, ranges of up to $1-2 \mathrm{~km}$ have been recorded (Lewis \& Ward 1987). Only the females are blood sucking - blood is essential for egg development. They bite at dusk, their approach is silent, in contrast to that of most mosquitoes, and the exposed parts are usually attacked. Their minute eggs are laid in cracks and crevices in dark, moist sites. They hatch after 9 to 12 days and go through 4 larval stages followed by a pupal stage before finally developing into a fly. Under favourable conditions the life cycle takes about one month but it may be considerably prolonged under adverse circumstances (Burgess 1981). Temperature is the only factor that correlates well with sandfly distribution throughout the world. Although they are able to survive cold in diapause (a period of suspended development accompanied by greatly reduced metabolism), larval development and adult activity are greatly reduced at temperatures below $20^{\circ} \mathrm{C}$. Therefore, the distribution of sandflies is largely confined to areas which have at least one month with a mean temperature of $20^{\circ} \mathrm{C}$ (WHO 1984).

Leishmaniasis is transmitted by sandflies of the genus Phlebotomus in the Old World and Lutzomyia or Psychodopygus in the New World. They lacerate the skin of an infected host to feed upon a pool of serosanguinous exudate containing amastigotes. The amastigotes develop into promastigotes in the sandfly gut before migrating to the salivary glands from which they can be regurgitated via the proboscis during the next blood meal. Development in the sandfly takes 7 - 10 days. During feeding saliva is introduced into the host and it has been shown that lysates of the salivary gland of Lutzomyia longipalpis greatly enhance the infectivity of L.major in mice. A sandfly injects about $10-100$ parasites during a blood meal. However, if a mouse is 
inoculated with this number none survive unless sandfly saliva is also injected - approximately $10^{5}-10^{6}$ parasites are needed to establish such a laboratory infection (Lerner 1993). In addition salivary gland lysate promotes faster development and growth of lesions - the combination produces a 5-10 fold increase in the size of lesions in murine foot pads and a 5000 fold increase in parasite numbers compared with injected parasites alone (Ribeiro et al 1989). These lysates have been analysed and a very potent vasodilator 'maxadilan', which resembles the mammalian neuropeptide calcitonin gene related peptide (CGRP), has been isolated. This is able to inhibit the production of hydrogen peroxide by macrophages in response to interferon gamma - the mechanism by which hosts destroy intracellular parasites (Theodos L1991).

Reservoir Hosts. Leishmania are maintained indefinitely within an ecological system consisting of one or a small number of sandfly vectors and one, or a few, vertebrate reservoir hosts. There is usually one primary reservoir host for a given species of leishmania within a particular focus, but other animals in the same area may become infected forming secondary hosts (from which leishmania can be transmitted to other mammals) or incidental hosts (from which leishmania are not passed on). These infections are often subclinical with very few amastigotes in the skin or viscera associated with a minimal host immune response. By contrast in dogs, which form the natural reservoir for visceral leishmaniasis, the parasites are abundant in both viscera and skin (from which they are readily picked up by sandfly vectors) and the infection is ultimately fatal. The site of leishmania in the host does not necessarily correspond to the site of the disease in man - for example L.b.guyanensis infects the internal organs of the reservoir sloth with minimal skin involvement, but it produces cutaneous disease in man (WHO 1990).

The leishmaniases can be crudely separated into two groups according to their reservoir hosts. The zoonotic leishmaniases such as L.mexicana mexicana $(\mathrm{Lmm})$ and $\mathrm{L}$. major in which the reservoir hosts are wild animals (e.g. foxes or jackals), commensals (e.g. rodents) or domestic animals (e.g. dogs); and the anthropophilic leishmaniases, such as L. tropica, in which man is the reservoir. However such distinctions are not absolute - L.tropica infection may derive from rodents or dogs and man may constitute a 
secondary reservoir for L.major. The principle reservoir hosts are shown in table 1.1.

Factors Influencing Infection Frequency. Ecological factors are paramount in determining the transmission of leishmaniasis and therefore there is great variation from place to place. Clearly man must be exposed to the bite of an infected sandfly for infection to occur. In the New World where leishmaniasis is primarily zoonotic, maintained by forest rodents, the disease is often an occupational one occurring more often in men, for example, during construction projects, hunting or forestry (Garnham \& Lewis 1959, Lainson \& Stangeways-Dixon 1963, Weigle et al 1993a, Weigle et al 1993b, WHO 1990). In each of these activities workers enter the forests where they encounter infected sandflies at the same time as man-made changes upset the pre-existing zoonotic cycle. The chance of any individual becoming infected is largely due to the length of time he has been exposed - for example during the construction of the trans-Amazonian highway the prevalence of positive leishmanin skin tests amongst workers increased progressively with longer exposure times. Entering the forests at night is a particular risk factor. In addition mini-epidemics may occur if workers enter a 'hot-spot' i.e. a well-delimited microfocus where transmission is high as a result of favourable environmental conditions associated with high densities of both reservoirs and sandflies.

Cutaneous leishmaniasis is also predominantly a disease of the poor who have primitive housing and sanitation. The organic debris associated with piles of bricks and stones constitute potential breeding and resting sites for sandflies. These people frequently live at the edge of towns close to the natural sylvatic cycle. A large number of people sleeping in one room further attracts peridomestic anthropophilic sandflies (WHO 1990).

Periodic fluctuations in the number of cases is well recorded. Although sandflies are present all year round in the tropics, each species tends to have an annual cycle and transmission is greatest towards the end of that cycle when the maximum number of parous sandflies are present. There are also marked year-to-year variations - in a prospective study in Três Braços, Brazil a peak occurred every 8-10 years (Jones et al 1987). 
Cutaneous leishmaniasis is increasingly seen in travellers returning to nonendemic areas, particularly those who have engaged in field work in forested areas (Herwaldt et al 1993). It is a major problem for the military who, by the nature of their task, bring in large numbers of non-immune individuals to endemic areas, where they often live under primitive conditions. The British garrison in Belize has reported 306 cases between 1978 and mid-1990 and the Walter Reed Army Medical centre in Washington treated 70 cases from Panama between 1977 and 1984 (Ballou at al 1987). In both cases the soldiers had been training in the jungle. It is currently a major problem for the Colombian army who are involved in operations against anti-government guerillas and drug barons; they have 2000 new cases of CL each year ( Lt Col Soto, personal communication). During the war between the Islamic Republic of Iran and Iraq thousands of cases occurred amongst soldiers of both sides It was the most common cause of casualty evacuation of Iranian soldiers during the early years of that war (Dowlati, personal communication).

\subsubsection{Pathogenesis, Pathology and Immunology}

Inoculated promastigotes (bearing surface antigens of 116 kdaltons) bind $C_{3 b}$ receptors on the macrophage and are phagocytosed, whilst immature promastigotes are destroyed by complement-mediated cytotoxicity (Kubba \& Al-Gindan 1989). Once inside the macrophage, the promastigotes lose their flagellae and become amastigotes which multiply repeatedly by binary fission. Infected macrophages then burst, releasing their amastigotes which in turn infect other macrophages (figure 1.3). The subsequent fate of the amastigotes depends upon parasite and host factors which are poorly understood. Viscerotrophic species, such as L.donovani, tend to migrate throughout the reticulo-endothelial system, whereas dermotrophic species, such as L.major, usually remain close to the inoculation site. Any spread of dermotrophic species tends to be late and only to adjacent skin (producing satellite lesions) or to lymphatics and regional lymph nodes. It is possible that dermotrophic species migrate as far as viscerotrophic species but are unable to survive in the internal milieu - for example L.donovani amastigotes are able to tolerate higher temperatures and resist complement-mediated cytotoxicity. 


\section{Figure 1.3: The life cycle of leishmania}

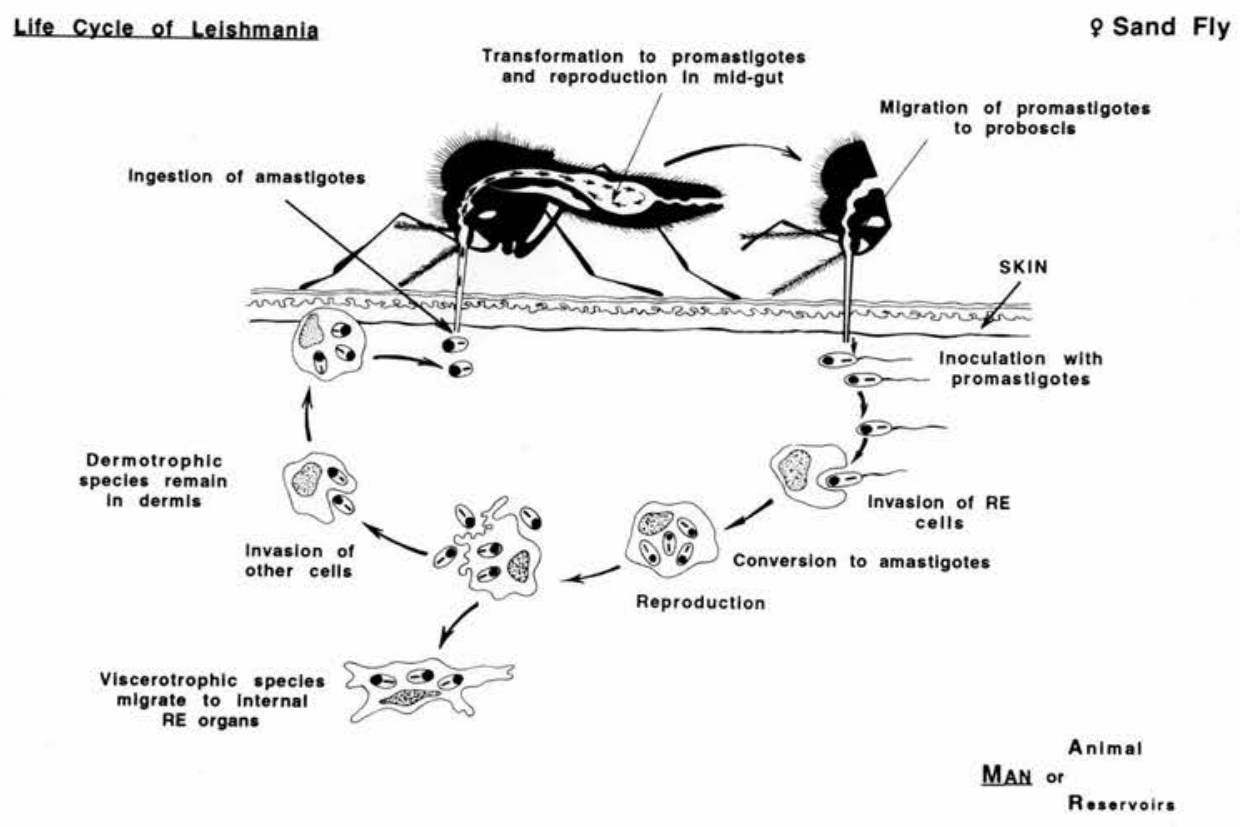

These phenotypic distinctions are not absolute. L.braziliensis braziliensis (Lbb) is able to migrate to the oropharyngeal mucosa where it may remain dormant for many years before reactivating to cause the destructive mucocutaneous form of the disease 'espundia'; there are reports of L.tropica causing visceral leishmaniasis, and in post-kala-azar dermal leishmaniasis (PKDL) the viscerotrophic parasite becomes dermotrophic as a consequence of treatment (Kubba \& Al-Gindan 1989, Magill et al 1993).

The interaction between the cell mediated immune response (CMI) of the infected subject and the amastigote is complex and results in a range of clinical and pathological changes. In all forms of leishmaniasis, however, the presence of amastigotes within the cells of the mononuclear phagocytic system remains the hallmark of the disease, although at times they may be difficult to detect. Infected macrophages measure 20-30 um in diameter whereas amastigotes (known as Leishman-Donovan bodies) are round to oval structures measuring only 2-5um (figure 1.4). They are surrounded by a plasma membrane and contain both a relatively large, deeply basophilic, 
nucleus and a smaller, densely staining, rod-shaped kinetoplast of extranuclear DNA (from the base of the flagellum which is lost in the conversion from promastigote to amastigote). Although haematoxylin and eosin (H\&E) will demonstrate amastigotes, a Giemsa stain is preferred by many - it stains the flagellum bright red (Kalter 1989, Lever 1990).

\section{Figure 1.4: A skin biopsy from Old World CL showing LD bodies.}

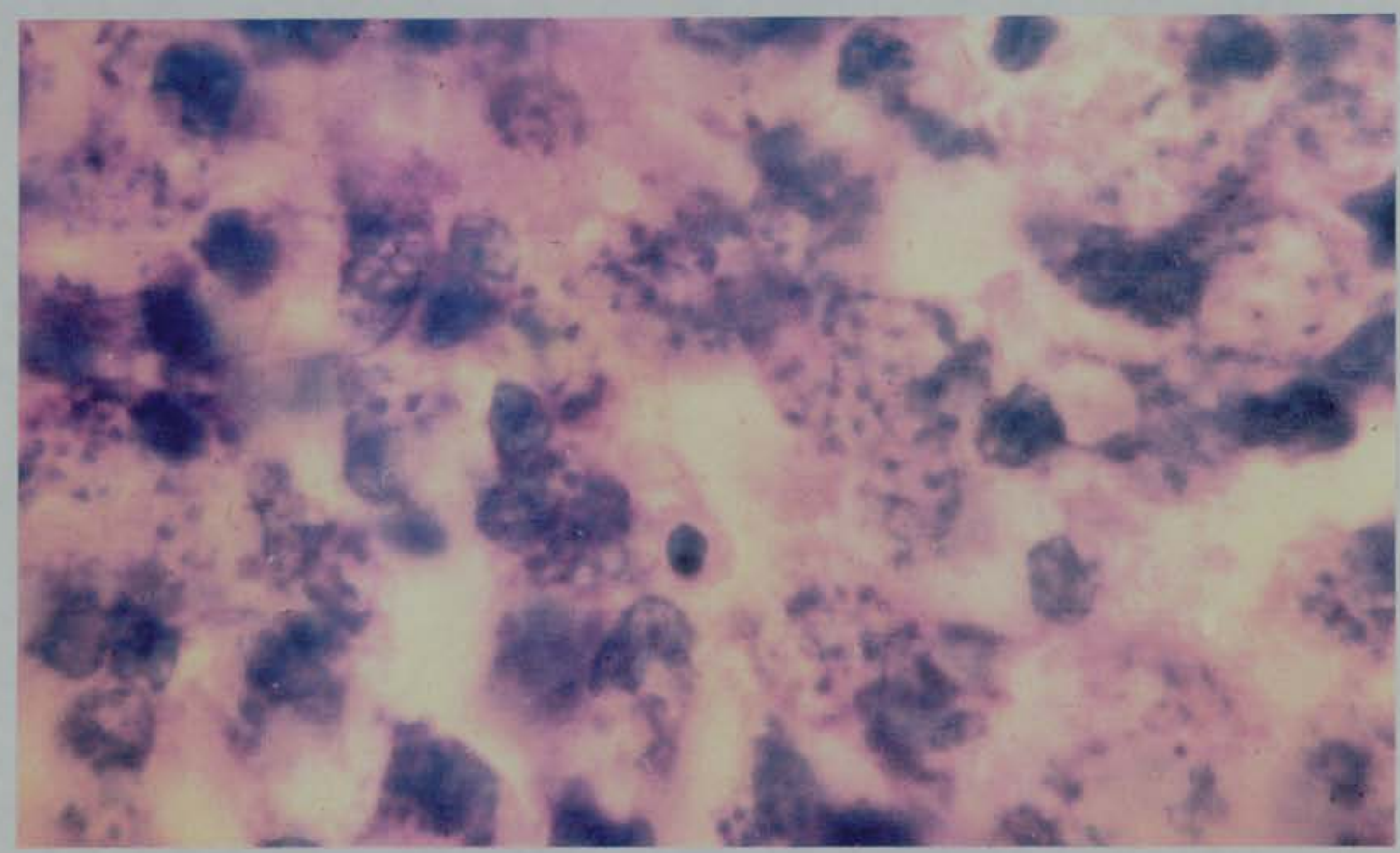

In early cutaneous lesions, the dermal infiltrate consists predominantly of macrophages filled with amastigotes. There are relatively few lymphocytes and plasma cells. As the lesions develop more lymphocytes and plasma cells appear and the superficial dermis becomes oedematous. The overlying epidermis becomes hyperkeratotic and subsequently breaks down to form an ulcer covered with a coagulum of hyperkeratotic debris, dried exudate, dead cells and a mixture of live and dead organisms. Over the following months, there is a gradual decrease in the number of amastigotes and macrophages, leaving a granulomatous infiltrate consisting of lymphocytes, epithelioid cells and multinucleate giant cells. At this stage it may be difficult or impossible to detect organisms in H\&E or Giemsa stained sections. Parasite elimination usually follows destruction of the macrophages, either at the centre of the circumscribed clusters in the dermis, with the release of amastigotes, or in the 
subepidermal zone causing liquefaction of the basal layer and ulceration. Polymorphs are present in the necrotic zone and lymphocytes are numerous at the periphery. Ultimately, either the patient's immune response is able to eliminate the infection and effect a spontaneous cure, or it fails and a chronic form of cutaneous leishmaniasis develops. Resolution occurs by fibrosis (WHO 1990, Kubba \& Al-Gindan 1989, Kalter 1989).

There are two special non-healing forms of $\mathrm{CL}$ which, although rare, are important because they represent two extremes of host cell-mediated immune response to infection with leishmania and have given insights into the pathogenesis of this disease. Leishmaniasis recidivans (or lupoid) is characterised by healing with fibrosis in the centre of the lesion, but failure to heal peripherally where there is a granulomatous infiltrate composed of epithelioid cells, multinucleate giant cells, a variable number of plasma cells but relatively few lymphocytes. Amastigotes are very scanty. The appearances are reminiscent of lupus vulgaris both histologically (although there is no caseation) and clinically (even apple jelly nodules are seen). The lesions usually occur on the face and develop from an acute lesion after an interval of $12-18$ months. There is a central fibrotic scar surrounded by an active edge consisting of papules and nodules. In this type the parasite persists indefinitely despite an exaggerated host $\mathrm{CMI}$ response. It is found mainly in Iran and Iraq. The other extreme is represented by diffuse cutaneous leishmaniasis (DCL) in which there is a specific defect in the host $\mathrm{CMI}$ response to leishmania. Lesions contain many macrophages full of amastigotes but lymphocytes are scarce. Clinically, the initial lesion spreads locally and then the disease disseminates to other areas of the body. There are numerous discrete papules and nodules which coalesce to form plaques scattered over the whole skin surface but they are particularly numerous on the face and the outer aspects of the limbs (figure 1.5). DCL is associated with L.aethiopica in the Old World and L.mexicana complex in the New World - there is an unusual focus in the Dominican Republic where all cases of $\mathrm{CL}$ are manifest as DCL. It is very resistant to treatment (Lever 1990, Ridley 1987, Kubba \& Al Gindan 1989, Griffiths 1987, Bryceson 1992, WHO 1990). 
A histological spectrum has been identified, rather analogous to that seen in leprosy, ranging from anergic forms of infection with heavily parasitized macrophages to hypersensitive forms with scanty organisms and a Figure 1.5: Diffuse cutaneous leishmaniasis.

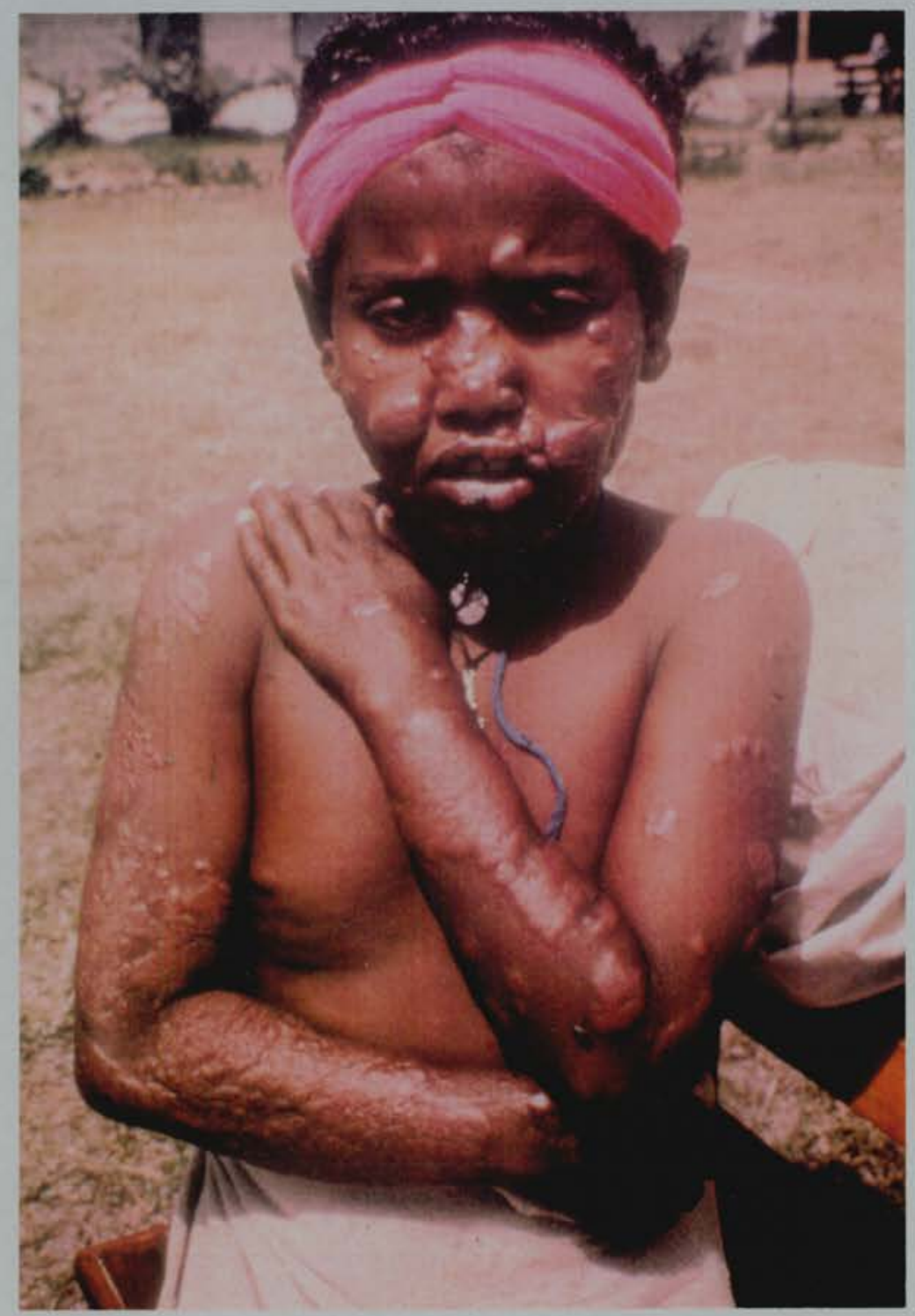


tuberculoid response. This spectrum has been divided up into 5 , broadly distinct, groups by Ridley (table 1.2).

\section{Table 1.2}

Ridley's Classification of the histopathological response in cutaneous leishmaniasis

GROUP 1 Diffuse macrophage granuloma formation. Macrophages are full of amastigotes but do not show signs of activation. Occasional lymphocytes and plasma cells, but no giant cells. Ulceration is rare.

GROUP 2 Focal macrophage granuloma formation, with central necrosis, surrounded by lymphocytes and plasma cells. Initially moderate numbers of organisms are present in surviving macrophages but latter these disappear. Ulceration may occur independently of the necrotic granuloma.

GROUP 3 The most non-specific group. A mixed inflammatory response consisting mainly of lymphocytes and plasma cells but with a few macrophages. Amastigotes scanty but are best seen in the sub-epidermal zone.

GROUP 4 Giant cells of foreign body and Langhans types are present together with a heavy lymphocytic infiltrate. Scanty or absent amastigotes.

GROUP 5 Tuberculoid response. Epithelioid cell granulomas with many giant cells but small numbers of lymphocytes and plasma cells. No necrosis. No amastigotes. 
The 5 groups are arranged in order of decreasing parasite load and include the whole spectrum of CL: DCL represents an extreme of group 1 anergy and recidivans an extreme of group 5 hypersensitivity. These are analogous to lepromatous and tuberculoid leprosy respectively. However, whereas in leprosy there is a straight-line spectrum from anergy to hypersenstivity, in individual cases of $C L$ there are aberrations in which the parasite load is out of step with the host cell response.

Immunology. The development of long-standing resistance to re-infection is the principal immunological feature of classical leishmaniasis. Re-infection amongst residents in regions endemic for L.tropica is uncommon and experimental infections in human volunteers confirm that resistance develops after recovery provided the initial infection runs its full course. However, immunity is not always complete: there are reports of re-infection occurring five or more years after an initial cure or as the result of steroid therapy. In one case re-infection by the same L.major type followed spontaneous healing of the original lesion although there were fewer parasites and the lesion healed more rapidly than the primary infection (Mauel \& Behin 1987).

It is the $\mathrm{CMI}$ response that provides resistance to infection with leishmania (Liew 1989). Although specific antibodies can be detected in the majority of patients with $\mathrm{CL}$ they do not appear to have a role in either the clearance of the intracellular organisms or in the establishment of immunity to re-infection even when present in high titre (Kalter 1989, Locksley \& Louis 1992). The $\mathrm{CMI}$ response can be demonstrated clinically by a delayed hypersensitivity test known as the leishmanin skin test (see diagnosis) which usually becomes positive within a few weeks of infection and then remains so for decades. The significance of this long-lasting skin reactivity is not clear. It may represent genuinely long-lived immunological memory or it may be explained by the existence of an occult infection in recovered individuals. It does not, however, represent a measure of resistance to re-infection. Whereas the reaction generally becomes positive early in the course of the disease, the lesion heals many months later. Re-infection of an individual with both an active sore and a positive skin test leads to the development of a typical ulcer that develops to the same stage as the original sore and they then heal together (Mauel \& Behin 1987). 
This sole dependence upon $\mathrm{CMI}$ for resolution has led to the intensive study of leishmanial infection. Most experiments have utilized in-bred strains of mice infected with L.major. T-helper $\left(\mathrm{CD}^{+}\right)$cells are thought to be important in mediating the spectrum of disease seen pathologically and clinically. Functional subsets of T-helper cells, Th-1 and Th- 2 cells, have been identified on the basis of their cytokine secretion profiles: Th-1 cells are thought to mediate the CMI response, they secrete interferon (IFN)-gamma and IL-2 and are associated with resistance to infection. Th-2 cells in contrast mediate specific antibody production, they secrete IL- 4 and IL- 10 and are associated with susceptibility to infection (Miller \& Scott 1989, Locksley \& Louis 1992). These observations have been followed up by blocking IFN-gamma and IL-4 with monoclonal antibodies (mAbs) in infected mice: strains of mice genetically resistant to infection with leishmania treated with anti-IFN-gamma $\mathrm{mAb}$ developed progressive infections. Treatment of susceptible strains with anti-IL-4 $\mathrm{mAb}$ resulted in attenuated disease and resolution of the lesions in $85 \%$ with the establishment of protective immunity. These findings led to clinical trials of immunotherapy using human recombinant cytokines. In a controlled study, intralesional treatment with recombinant-IFN-gamma produced a reduction in size of 12 out of 13 lesions due to L.b.gyanensis after 3 weeks and some lesions were free of parasites. Similarly 9 out of 13 lesions due to L.tropica resolved completely following 4-8 weeks treatment. Resolution was associated with increased numbers of IL-2+ T-cells indicating that therapy with recombinant-IFN-gamma promoted healing of $C L$ by enhancing the CMI response (Gran \& Modlin 1991, Harms et al 1989).

There are, however, few $\mathrm{T}$ cells in the dermal infiltrate in early lesions so infected macrophages, expressing parasite antigens, are unlikely to meet $\mathrm{T}$ cells with the corresponding specificity required to trigger the $\mathrm{CMI}$ response. Epidermal Langerhans cells express receptors for $\mathrm{C} 3 \mathrm{~b}$ which opsonizes leishmania parasites for macrophages. It has been suggested that epidermal Langerhans cells migrate from the epidermis to the dermis and, like macrophages, take up parasites following which they express parasite antigens in association with MHC class II molecules on the cell surface. A proportion of Langerhans cells may transport parasites from the infected skin to the draining lymph node for presentation to antigen-specific resting $T$ cells. 
As a result, activated $\mathrm{T}$ cells migrate via the blood into the lesion where infected macrophages and Langerhans cells that remain in the dermis regulate their effector activity by several mechanisms including cytokine secretion (Moll 1993).

\subsubsection{Clinical Features}

The clinical features of $C L$ differ between different regions of the world, reflecting differences in the species of the parasite, the sandfly vector and the genetic, immunological and cultural background of the patients (WHO 1990). The principle cutaneous manifestations are summarised in table 1.3. The lesions develop where the infected sandfly bites and are therefore usually on the exposed parts of the body. A cluster of lesions may occur and this has been attributed to repeated probings by the sandfly which has difficulty aspirating blood through its promastigote-laden proboscis. The incubation period is $1-12$ weeks, tending to be shorter with a larger inoculum (Kalter 1989). Although each parasite is classically associated with a distinctive clinical picture, the features overlap and they cannot be reliably distinguished on clinical grounds alone.

\section{Table 1.3}

\section{Cutaneous manifestations of leishmaniasis}

\begin{tabular}{clc}
\hline Acute disease & Chronic disease & Miscellaneous \\
\hline Old World CL & non-healing acute sore & Leishmanioma \\
wet/rural sore & mucocutaneous leishmaniasis & Post Kala-Azar \\
dry/urban sore & leishmaniasis recidivans & leishmaniasis \\
New World acute sore & diffuse cutaneous leishmaniasis & \\
& & \\
\end{tabular}

Old World acute CL (oriental sore). The lesion starts as an erythematous papule, rather like an insect bite with which it is frequently confused. It 
persists and enlarges to form a painless nodule with a surface crust resembling a volcano. In some cases there is a larger subcutaneous component which has given rise to the term 'iceberg nodule'. In most cases the crust separates to expose an ulcer (Kubba et al 1987a) (figure 1.6). Painless sporotricoid lymphatic spread is common and organisms can be found in the regional lymph nodes (Al-Gindan et al 1989, Kubba et al 1987b). Lesions heal by fibrosis leaving a sharply demarcated, irregular, cribriform scar which in coloured races is often hyperpigmented and disfiguring. Lesions, particularly in cases due to L.major, are often multiple and occasionally over 100 lesions occur in a single patient (Kalter 1989).

Figure 1.6: Old World cutaneous leishmaniasis.

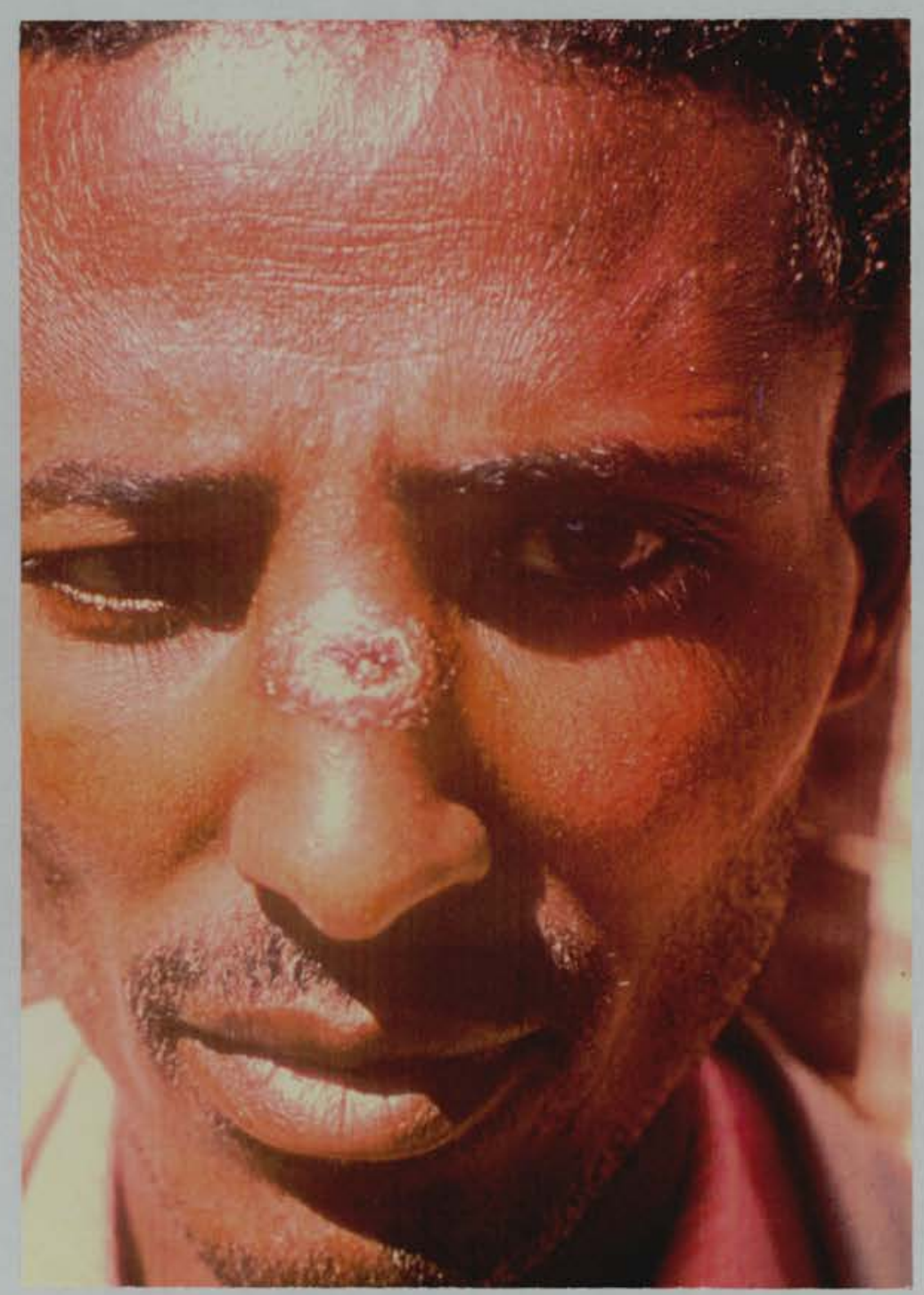


Oriental sores are traditionally divided into wet and dry types. The wet, rural or zoonotic, sore is a more inflammatory lesion which is classically associated with L.major. It is usually acquired in rural areas where the infecting organisms are primarily rodent parasites and are, therefore, poorly adapted to man. After an incubation period of less than two months, an erythematous papule develops and two weeks later a central crust appears which usually falls off revealing a bright red base consisting of slough alternating with papules of proliferating granulation tissue. The ulcer enlarges over the following two to three months reaching a diameter of $3-6 \mathrm{~cm}$. Multiple satellite papules which measure $2-5 \mathrm{~mm}$ and do not contain parasites are much more common in the wet than in the dry type. The lesion heals in 1.5 8 months (mean 3 months).

The dry, urban or anthroponetic, sore is a more quiescent but indolent lesion. It is classically associated with L.tropica and it is more commonly found in urban areas where the parasite is better adapted to man. The lesion starts as a brown nodule which enlarges to form a plaque measuring $1-2 \mathrm{~cm}$ in diameter in about six months. It then ulcerates in the centre and has a closely adherent crust. Healing generally takes 5 - 14 months (mean 10 months) but may take up to two years (Bryceson 1987, Griffiths 1987).

Lesions due to L.aethiopica are usually single and most commonly occur on the central part of the face. Satellite papules amalgamate into a large spreading nodule. Ulceration or crusting is not always a feature. There is relatively little inflammation and healing takes $2-5$ years. It may progress to DCL. L.infantum (which generally causes visceral leishmaniasis in children) usually gives rise to a self-healing sore in adults. Its appearance and evolution are slow compared to lesions caused by L.major (WHO 1990, Kubba et al 1987/, Griffiths 1987, Bryceson 1992).

\footnotetext{
New World acute CL. A typical lesion starts as an inflammatory papule which rapidly evolves to a deep, well circumscribed, ulcer with raised violaceous borders and a friable granulomatous base which may be covered by an eschar (figure 1.7). It can reach several centimetres in size within a month and heals with a characteristic scar (Jones et al 1987, Walton 1987).
} 
In an epidemiological study from Três Braços, an area of Brazil endemic for L.braziliensis, 369 patients were detected (64 with active disease

Figure 1.7: New World CL from Belize.
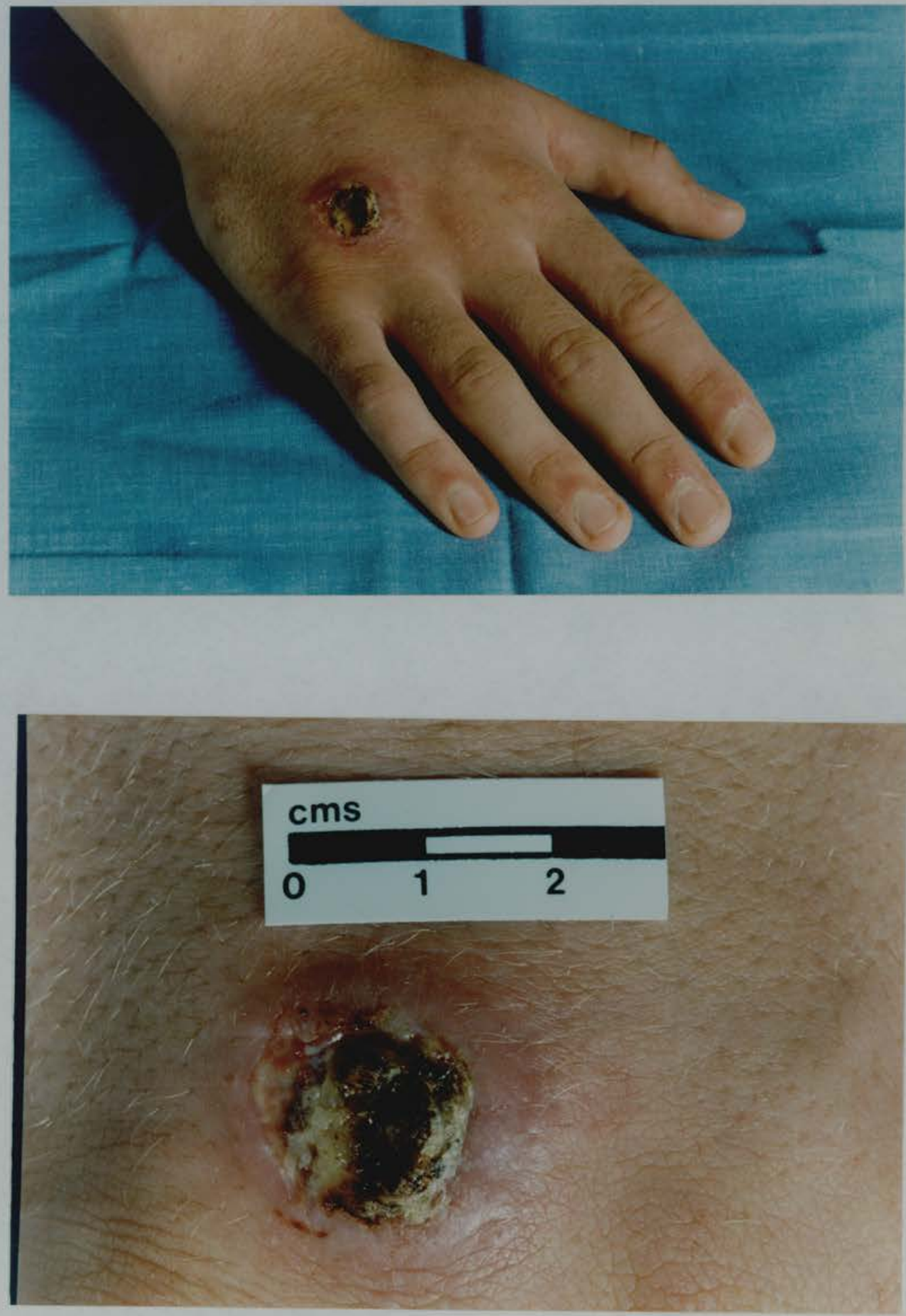
and the remainder with a typical scar indicating previous infection). A single lesion was present in $74 \%$, two lesions were present simultaneously in $17 \%$, three in $5.6 \%$ and four or more in $3.4 \%$. The leg was the most common site ( $73 \%$ of lesions) but lesions were also present on the arms (11\%), trunk $(10 \%)$ and on the head and neck $(6 \%)$. The mean size of the lesions was 33 $\mathrm{mm}$ (range: 5 to $>100 \mathrm{~mm}$ ) but it was not clear whether this referred to the size of the ulcer or to the diameter of the whole lesion. All ages were affected, but it was most prevalent in the 10-15 year age group, there was a male predominance $(1.46: 1)$. A second lesion at a different site was observed 1 to 20 years after the primary infection had healed in $11 \%$ of patients (Jones et al 1987). Spread and localisation by trauma has also been observed (Walton 1987). Involvement of the lymphatics is common in infections caused by L.b.panamensis and L.b.guyanensis - pea-sized subcutaneous nodules or chords ascend the lymphatics (Berger et al 1985). The ulcers are painless unless there is significant secondary bacterial infection (as distinct from colonization which is common).

The natural history of New World ulcers has been thought to be one of gradual healing over months or years (Berman 1988). However, two recent reports cast doubt on this belief, suggesting that spontaneous healing may occur within a few months particularly in cases caused by L.mexicana complex. In a placebo controlled study of sodium stibogluconate versus allopurinol from Equador, involving patients with both $\mathrm{Lmm}$ and $\mathrm{Lbb}$, spontaneous healing (defined as $80 \%$ re-epithelialization after 6 weeks observation) was observed in 9 out of 12 untreated patients (Guderian et al 1991). A subsequent prospective study of 48 patients from Guatemala showed that out of a total of 57 lesions ( 32 due to $\mathrm{Lbb}$ and 25 due to $\mathrm{Lmm}$ ) which had been present for a mean of 4.5 weeks, spontaneous healing (defined as complete re-epithelialization, absence of inflammation and remaining healed during at least 6 months observation) occurred in $68 \%$ of those due to $\mathrm{Lmm}$ but in only $6 \%$ of those due to Lbb. In those that did heal, the median healing time was 14 weeks for Lmm and 13 weeks for Lbb (Herwaldt et al 1992). The natural history of New World CL merits further study. 
Chronic Cutaneous Leishmaniasis. Lesions that have been present for over one year are considered chronic. The most common form of chronic CL is an acute sore that has failed to heal. Leishmaniasis recidivans and diffuse cutaneous leishmaniasis have already been described (see pathogenesis). Mucocutaneous leishmaniasis (MCL) - 'espundia' - is most often observed in the New World (figure 1.8). Although mucous membrane disease can occur

Figure 1.8: Mucocutaneous leishmaniasis.

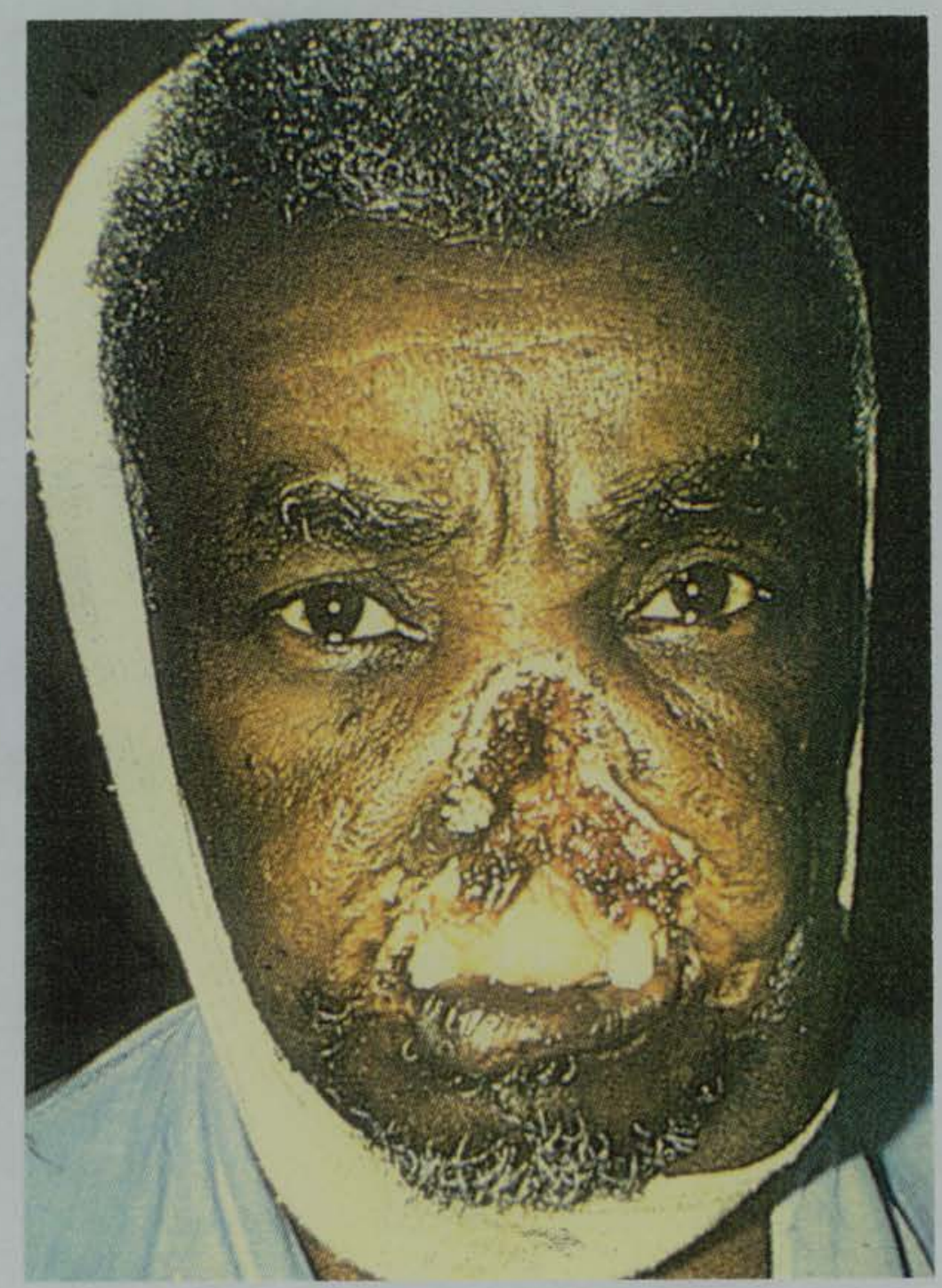


in the Old World, due to contiguous spread of facial infections with L.aethiopica, and oropharyngeal granulomata of L.donovani are seen in the Sudan, neither of these closely resemble espundia clinically. Lbb is the most common cause of MCL but L.b.panamensis and L.m.amazonensis have occasionally been isolated from the lesions (Kalter 1989). The disease usually starts in the nasal mucosa with septal perforation, and it then progresses to destroy the remainder of the nose, upper lip, pharynx and larynx. The rate of progress and the amount of destruction varies from patient to patient. A history of nasal blockage, epistaxis, discharge or nasal ulcers is often obtained but, unlike leprosy or syphilis, which erode the proximal nasal cartilage, MCL has a predilection for the distal cartilaginous portion causing the characteristic 'tapir nose'. The nose and surrounding face may become very swollen. Laryngeal involvement causes hoarseness, stridor and a brassy cough followed by problems with swallowing and respiration. Death usually results from secondary infection, starvation or laryngeal obstruction. Dissemination is thought to be via the blood stream (Kalter 1989). MCL occurred in $2.7 \%$ of individuals infected with Lbb in Brazil. It was more common in males $(2.3: 1)$, in those in whom the primary skin lesions had been either large or multiple, in those whose skin lesions had persisted for over a year and in those who had recovered after inadequate therapy for the primary lesion. The median time from the onset of the primary skin lesion to the recognition of mucosal disease was 6 years (range: $0-16$ years) (Jones et al 1987). It is often difficult to isolate the organisms from the mucosal lesions (Kalter 1989).

\subsubsection{Diagnosis}

The diagnosis of leishmaniasis is often made on the basis of the appearance of the lesion in conjunction with an appropriate history of exposure to the infection. However, because the differential diagnosis is extensive (table 1.4), pathological confirmation should always be obtained (preferably by observing the organism in tissue and/or culturing it). Unfortunately this is not always possible in practice. In a study of 475 cases due to L.major in Saudi Arabia the parasite was demonstrated by smear in $50-80 \%$ (depending on the investigator and the technique used), by skin biopsy in $70 \%$ and by culture in 
Table 1.4

Differential diagnosis of cutaneous leishmaniasis

\section{Acute}

Insect bite

Trauma

Foreign body granuloma

Pyogenic granuloma

Leprosy

Tropical ulcer

Impetigo

Furuncle

Carbuncle

Ecthyma

Anthrax

Orf

Tularaemia

Milker's nodule

Swimming pool granuloma

Syphilitic granuloma

Yaws

Sporotrichosis

Blastomycosis

Kerion

Molluscum contagiosum

Viral warts

Myiasis

Dracunculosis

Keratoacanthoma

Basal cell carcinoma

Squamous cell carcinoma

Lymphoma

Leukaemia

\section{Chronic}

Keloid

Lupus vulgaris

Syphilitic gumma

Sarcoidosis

Granuloma faciale

Discoid lupus erythematosis

Jessner's lymphocytic infiltrate

Lymphoma

Wegener's granulomatosis 
only $50 \%$. If all 3 methods were combined the parasite was still not detected in $10-20 \%$ of cases (Kubba et al 1987a). A similar study from Colombia compared 7 diagnostic methods in a group of 177 patients in whom a clinical diagnosis of CL (165 patients) or MCL (12 patients) had been made. The combined overall detection rate of all 7 methods was $67 \%$ to which each individual method contributed: histopathology $14 \%$, impression smear $19 \%$, dermal scraping $22 \%$, aspirate \& culture $58 \%$, aspirate \& hamster inoculation $38 \%$, biopsy \& culture $50 \%$ and biopsy \& hamster inoculation $52 \%$. Similar results were obtained in a study from Guatemala (Navin et al 1990). It was most difficult to detect parasites in older lesions whichever method was used (Weigle et al 1987). Specimens should be taken from the active edge of the lesion as that is where organisms are most numerous. Diagnostic methods which have been described include:

Skin biopsy. A wedge or punch biopsy, taken under local anaesthetic, can be used for making impression smears, parasite cultures and for routine histological examination. The area should be thoroughly cleaned with $6 \%$ hydrogen peroxide to reduce bacterial contamination before taking the biopsy. Amastigotes (LD bodies) can be identified in routine H\&E sections during the early stages of the disease but they become less numerous with the passage of time and may be impossible to find after a few months. The typical granulomatous histology is a much less specific diagnostic pointer. At that stage it may be possible to detect amastigotes in formalin-fixed specimens using polyclonal antibodies in conjunction with immunoperoxidase staining (Sells \& Burton 1981). Monoclonal antibodies have been reported which, when used in conjunction with immunofluorescent labelling, can demonstrate as few as 50 amastigotes in both smears and biopsies; this technique can confirm the diagnosis in as little as 1-2 hours (Anthony et al 1987). DNA probes have also been developed for many species of leishmania which are able to detect 100 - 200 organisms in a impression smear and are used in some centres in the USA (Wirth 1992).

Smear preparations. Impression smears are made by gently pressing a skin biopsy against a glass microscope slide 2 - 3 times before fixation. Alternatively, smears can be prepared using tissue juice obtained by aspirating the edge of the lesion with a 20 gauge needle or by using a slit-skin 
technique akin to that used in leprosy. In each case the smears should be allowed to dry prior to fixation in $95 \%$ alcohol for 30 seconds. Amastigotes may be detected both intracellularly and extracellularly (Berger et al 1987).

Culture of leishmania. This is often difficult and involves both art and science. Whilst some species, such as $\mathrm{Lmm}$, grow readily others, such as $\mathrm{Lbb}$, are more fastidious. Essentially the tissue biopsy is placed in a biphasic blood agar medium such as a modified NNN, to which antibiotics are added to prevent bacterial overgrowth, and incubated at $22^{\circ} \mathrm{C}$. Promastigotes may be identified in as little as 7 days, although it often takes up to 21 days. The techniques for culture (and also isoenzyme analysis) are described in a handbook by Evans (1989). Animal inoculation is a useful alternative to in vivo culture, particularly when the parasite load is low or if sterile conditions are difficult to achieve. The golden hamster is generally the most suitable as it is sensitive to most strains of leishmania, although the Balb/c mouse is best for L.major. Homogenised tissue containing amastigotes is injected intradermally into the nose, ears or foot pads and the inoculation site is biopsied 7-10 days later to obtain samples for culture or, at a later date, for histological examination (Manson-Bahr 1987).

Identification of Leishmania species. Isoenzyme analysis remains the principle method of typing because it is simple to use, reproducible and well established. It is the standard against which newer methods, such as monoclonal antibodies and DNA probes, are assessed. An aqueous extract containing many enzymes is prepared from promastigotes cultured from the lesion. This extract is analysed by electrophoresis during which the banding pattern, identified by staining for a single enzyme such as glucose-6dehydrogenase or malic dehydrogenase, is compared with that of a known reference strain. Several such zymogrammes, each stained for a different enzyme, are required to identify a given species (Kreutzer \& Christensen 1980, Kreutzer et al 1987, Evans 1989). These reference strains are maintained at several WHO international leishmania reference centres such as that at the London School of Hygiene and Tropical Medicine.

DNA buoyant density has also been used to distinguish between leishmanial strains. This method is based on the observation that the equilibrium buoyant 
densities vary with the guanine and cytosine content of the DNA coupled with theoretical estimates of DNA homologies as revealed by their buoyant densities - for example a buoyant density difference of $0.001 \mathrm{~g} / \mathrm{ml}$ suggests significant differences in $10-25 \%$ of their genes. Buoyant densities range from $1.716-1.720 \mathrm{~g} / \mathrm{ml}$ for nuclear DNA (nDNA) and $1.688-1.707 \mathrm{~g} / \mathrm{ml}$ for kinetoplast DNA (kDNA). The kDNA therefore varies more than the nDNA between species (Janovy 1987). These methods have now been superseded by advances in recombinant DNA technology.

Monoclonal antibodies have been developed which are able to distinguish between different species of leishmanial promastigotes but probes are not yet available for the specific identification of amastigotes. Monoclonal antibodies have the advantage of speed and the available panel is increasing but it still lacks the sensitivity and specificity of isoenzyme analysis (McMahon-Pratt \& David 1981, Hanham 1991).

DNA probes for kDNA are being developed which can identify the species of cultured promastigotes, and in some cases, tissue amastigotes (Barker et al 1986, Wirth \& McMahon-Pratt 1982, Lopez et al 1988). The early techniques used radio-labelling which limited their application to research laboratories. Alternative markers, such as biotin and digoxigenin, are currently under assessment, but in general they appear less sensitive. Chemiluminescent, rather than colourimetric, markers may improve sensitivity (Wilson 1991). Most recently the polymerase chain reaction has been utilised to detect species specific DNA sequences for $\mathrm{Lbb}$ and this holds great promise for the future (de Bruijn \& Barker 1992).

Serological methods. Techniques such as the enzyme-linked immunosorbent assay (ELISA) and the indirect fluorescent antibody test (IFAT) have been widely used in the diagnosis and management of visceral leishmaniasis. However, in cutaneous disease, the antibody titres are much lower and in Old World disease serology is not useful in diagnosis (WHO 1990). More encouraging results have been reported in New World disease with both IFAT (Pappas et al 1983) and ELISA (Guimaraes et al 1990) although both groups reported a false positive rate of $5 \%$. In a group of British soldiers who contracted CL in Belize, an ELISA test was insufficiently 
sensitive and specific to be of use (Evans, personal communication).

Western blotting can detect leishmanial antigens in the serum of patients with multi-lesional disease and future developments in this area can be expected (Bogdan et al 1990). Walton (1980) speculates, that in those who do have a significant antibody titre and in whom the diagnosis is proven, if the high titre persists then that is indicative of a persisting infection. It may, therefore, be an indication for re-treatment.

Leishmanin (Montenegro) skin test. This detects delayed-type hypersensitivity and is analogous to the tuberculin and lepromin tests. A suspension containing $10^{6}$ promastigotes $/ \mathrm{ml}$ in $0.5 \%$ phenol-saline is used; $0.1 \mathrm{ml}$ is injected intradermally into the forearm. An equal volume of $0.5 \%$ phenol saline is injected into a second adjacent site as a control to detect sensitivity to the diluent. An indurated nodule with surrounding erythema develops and the reaction is read at $48-72$ hours. An area of induration measuring $5 \mathrm{~mm}$ or more is positive (Manson-Bahr 1987). Unfortunately, because standardised test antigens are not available, the reagents are produced in the laboratories of tropical disease centres and vary in their sensitivity and specificity (Weigle et al 1991). The test becomes positive once the lesions begin to crust (Even-Paz \& Sagher 1961, Mauel \& Behin 1987) and it remains so indefinitely. It is therefore unable to distinguish between past and present infection. Its main use is in epidemiological studies because in endemic areas up to $9 \%$ of healthy controls are positive, presumably from past, often asymptomatic, infection (Weigle et all 1991). Its value in repeated assessment of the same individual, for example, before and after exposure is not known for each reagent; the test itself sensitise the individual and give a false positive reaction. It is, however, a useful supplementary test in visitors returning with a suspicious lesion.

\subsubsection{Treatment}

There is no single optimal treatment for all forms of CL. Indeed, even neglecting economic factors, the natural diversity of the parasites, together with the wide spectrum of clinical disease that they produce, means one is unlikely to evolve in the forseeable future. The situation is further complicated by the 
self-healing nature of $\mathrm{CL}$. Although this has been appreciated for many years in Old World disease it has only been recognised relatively recently that over half the lesions due to $\mathrm{Lmm}$ heal spontaneously within 4 months (Herwaldt et al 1992). Adequately controlled therapeutic trials are therefore essential to assess the efficacy of any new treatment. Unfortunately the literature abounds with reports of 'effective' treatments based on case reports and uncontrolled, small, series which makes it difficult to assess their efficacy. Selection of the most appropriate therapy can be very difficult. It has become increasingly apparent that each endemic area must establish its own optimum treatment regimen based on efficacy and toxicity, without neglecting the practical difficulties of administration and cost.

Fortunately there are some guiding principles. Lesions due to different species vary in both their severity and response to treatment - New World disease tends to be more severe and longer lasting than Old World disease. Most Old World lesions will heal spontaneously within a few months or at most two years, therefore active treatment is not mandatory. Indeed, for patients living in endemic areas, it may not be appropriate because spontaneous healing is associated with the development of protective immunity. However, patients with lesions on cosmetically or functionally important sites, such as the face or hands, those with associated lymphangitis and those with multiple or more complicated lesions, are best given active treatment. Local treatment is appropriate for early non-inflamed lesions and systemic therapy for those with multiple or more complicated lesions. An exception to these rules are those lesions caused by L.aethiopica which is relatively resistant to antimony and for which local treatments have not been validated. The WHO recommends that they are left to heal spontaneously unless they occur in the nasopharyngeal area, in which case systemic pentamidine should be used (WHO 1990).

In New World disease the first crucial step is to decide whether or not one of the L.braziliensis complex could be the infecting species because, until further information is available, all such cases must be considered capable of causing mucocutaneous disease and therefore adequate systemic therapy is mandatory. Hence, if the patient contracted $\mathrm{CL}$ in any area in which L.braziliensis occurs (i.e. Belize, Brazil, Colombia, Costa Rica, French Guiana, Honduras, Surinam, Panama, Venezuela, the western slopes of the Andes and 
the Argentinian highlands (Lainson \& Shaw 1987)), the parasite must be isolated and identified before any alternative to optimal systemic therapy is considered. If the disease were contracted in other areas, or if L.mexicana complex (except L.m.amazonensis) is isolated, then alternatives, including topical or no therapy at all, may be considered. The WHO recommends that cases due to L.m.amazonensis are treated in the same way as those due to Lbb because a high proportion develop DCL (WHO1990).

Table 1.5

Therapeutic Agents for Leishmaniasis

$\begin{array}{ll}\begin{array}{l}\text { Systemic } \\ \text { sodium stibogluconate } \\ \text { meglumine antimoniate }\end{array} & \begin{array}{l}\text { Topical } \\ \text { paromomycin sulphate15\% \& } \\ \text { amphotericin B }\end{array} \\ \begin{array}{l}\text { ketoconazole } \\ \text { itraconazole }\end{array} & \begin{array}{l}\text { antimony potassium tartrate } \\ \text { pentavalent antimonials }\end{array} \\ \begin{array}{l}\text { pentamidine } \\ \text { rifampicin } \\ \text { dapsone }\end{array} & \begin{array}{l}\text { clotrimazole } \\ \text { chlorpromazine } \\ \text { cryotherapy }\end{array} \\ \begin{array}{l}\text { monomycine methyluracil } \\ \text { cycloguanil pamoate }\end{array} & \begin{array}{l}\text { laser } \\ \text { allopurinol \& allopurinol } \\ \text { local heat }\end{array} \\ \text { ribinoside } & \text { Grenz rays } \\ \quad \text { bovine serum albumin } & \\ \text { liposome therapy with antimonials } & \\ \quad \text { or amphoteracin B } & \\ \text { immunotherapy with Lmm } & \\ \text { promastigotes and BCG } & \\ \text { interferon gamma }+/ \text { - antimonials } \\ \text { aminosidine (paromomycin) }\end{array}$

The literature is full of alternative therapies (table 1.5), some are of proven efficacy (eg pentavalent antimony and pentamidine), some are promising 
anecdotally or in small series (eg ketoconazole) and others are under investigation (eg paromomycin ointment). It is not feasible to describe each one in this thesis so I refer to several recent reviews (Bryceson 1987, Berman 1988, Mukherjee et al 1990, Olliaro \& Bryceson 1993) and I shall consider pentavalent antimonials and aminosidine (paromomycin) in detail.

\section{Pentavalent antimonials}

Pentavalent antimonials remain the mainstay of effective systemic therapy for all forms of leishmaniasis. Many different preparations have been developed over the years (table 1.6) and these vary in their antimony composition (as can Table 1.6

\section{Overview of pentavalent antimonials}

Pentavalent antimonials are derived from stibonic (antimonic)acid and fall into 2 groups:

(1) Derivatives of benzene (or para-aminophenyl) stibonic acid.

Polymerised 4-aminobenzene stibonic acid=stianilic acid.

Salts of stibanilic acid=stibonates.

(a) Sodium salts of stibanilic acid = Stibamine and Stibosan.

(b) Acetyl derivative of stibamine = Stibacetyn, Stibenyl.

(c) Diethylamine derivative of stibacetin $=$ ethyl stibamine $=$

Neostibosan

(d) Urea salt of stibanilic acid = urea stibamine $=$ Urea

Stibosol, Stiburea.

(e) Nitrogen glucoside of Stibamine $=$ Neostam .

(2) Derivatives of stibonic acid in which antimony is joined through

oxygen atoms to the carbon chain(s) of glucose.

(a) Sodium stibogluconate $=$ sodium antimony $(\mathrm{I})$ gluconate $^{*}=$

Pentostam, Solustibosan, Solyusurmin, Stibanate, Dibanate, Stibek.

(b) $\mathrm{N}$-methyl glucamine antimoniate $=$ meglumine antimoniate $=$ Glucantime, Prostib.

${ }^{*} A$ preparation of sodium antimony $(I)$ gluconate containing trivalent antimony was in use for a short time in the late 1930s. 
different sources of the same compound). Urea stibamine, Stibosan and Neostibosan became available in the 1920s. Pentostam was first manufactured between 1935 and 1945 and the concentrated solution (100mg antimony/ml) became available and was first used in 1945 (Berman 1988).

Two preparations are widely available commercially: sodium stibogluconate (PentostamR, Wellcome, UK), which contains $100 \mathrm{mg}$ antimony/ml and is primarily used in English speaking countries, and meglumine antimoniate (Glucantime R, Rhône Poulenc, France), which contains $85 \mathrm{mg}$ antimony $/ \mathrm{ml}$ and is principally used in French and Spanish speaking countries. Although their efficacy has been well established their precise structure and mode of action are not well defined. A commonly drawn structure for Pentostam shows 2 molecules of gluconate complexed via oxygen atoms to 2 molecules of antimony with 3 sodium atoms balancing the 3 negative charges on the rest of the molecule (figure 1.7). In reality it is likely to be a more complex macro-

Figure 1.7

Sodium stibogluconate (Pentostam $\mathbf{R}$ ) (James et al 1985)

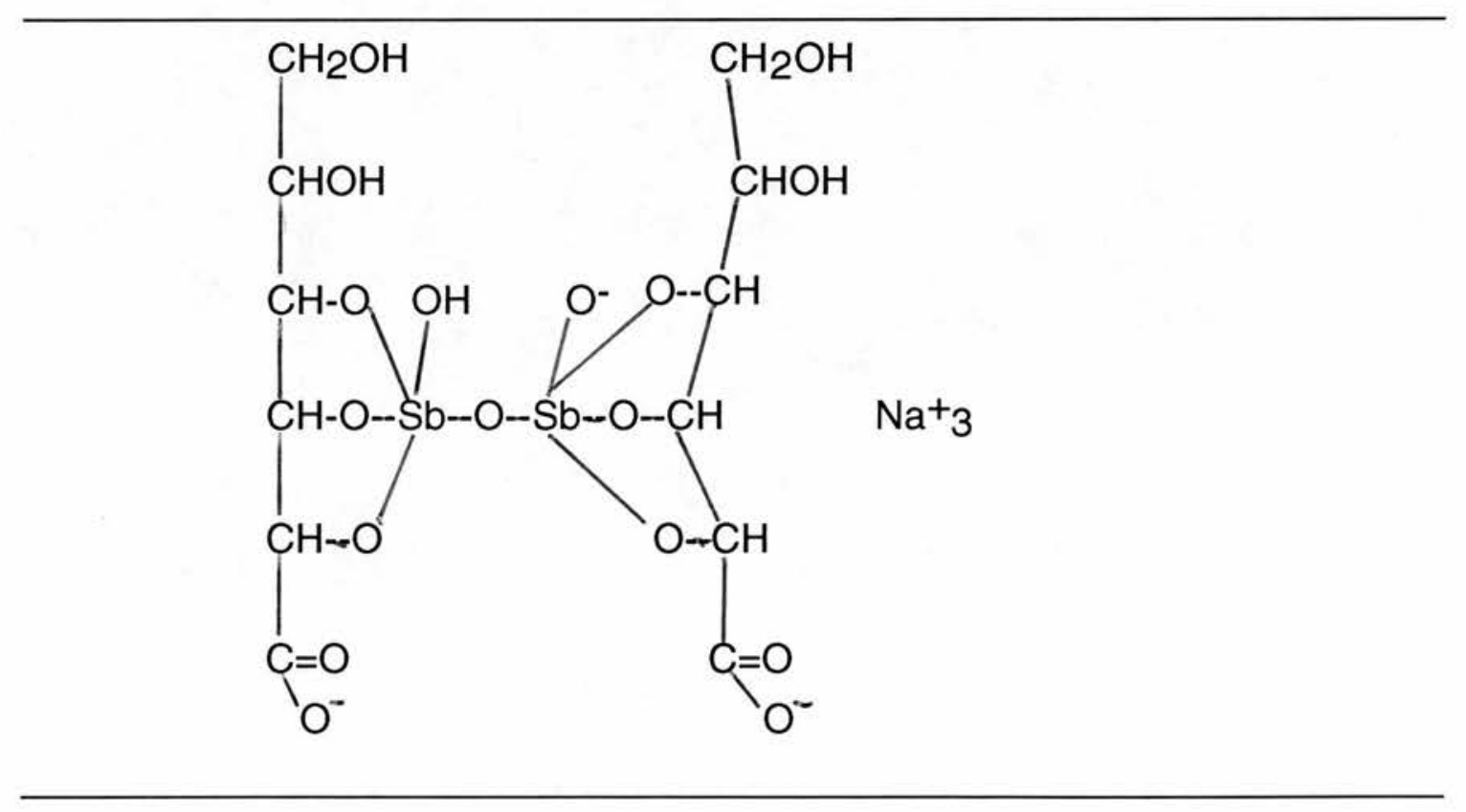

molecular structure because the molecular weight for that structure is 746 daltons and the osmolarity of a solution of $100 \mathrm{mg}$ of Sb/ml would be 1644 mOsmol. In fact the osmolarity of Pentostam is approximately $800 \mathrm{mOsmol}$ 
and it consists of numerous moieties with molecular weights of 100-4000 daltons. Clinical formulations of sodium stibogluconate may, therefore, be described as 'an unknown number of uncharacterised complexes of antimony with carbohydrates derived from gluconic acid' (Berman 1988). It would not be surprising if different sources differed in their composition in important respects, indeed the production of Pentostam $R$ has been described as 'cookery rather than chemistry' (Marsden 1985). In fact the osmolarity of Pentostam $R$ varies from batch to batch and if it is over $1000 \mathrm{mOsmol}$ then it is clinically ineffective (Berman \& Grogl 1988). The situation may be further complicated, at least for Glucantime $e^{R}$, because there is a variable amount (10-18\%) of (toxic) trivalent antimony present (Franco 1992).

These compounds probably have a number of modes of action on leishmanial amastigotes which include inhibition of bioenergetics by (1) the inhibition of glycolytic enzymes resulting in a decrease in ATP and GTP (and a consequent increase in ADP and GDP) but they do not affect the citric acid cycle or the hexosemonophosphate shunt, and, (2) the inhibition of fatty acid oxidation (Berman 1988). More speculatively, it may act as an immunomodulator whereby it in some way injures the amastigote, thereby exposing different antigens which trigger CD4 dependant destruction. In HIV infection where there are few functional CD4 cells sodium stibogluconate is ineffective or only effective at very high doses (Peters et al 1990, Magill personal communication).

It cannot be absorbed orally and therefore parenteral administration (im or iv) is necessary. It is rapidly excreted, largely unchanged, by the kidney - $80 \%$ of a single dose is eliminated within 6 hours (Dollery 1991). Whilst this avoids acute toxicity, about $5 \%$ is excreted as trivalent antimony, which has a much longer half-life, and may account for the observed cumulative toxicity which increases in proportion to the dose and duration of therapy (Bryceson 1987).

Efficacy. The efficacy of pentavalent antimonials in CL has been established beyond doubt provided they are given in adequate doses and for an adequate length of time (table 1.8). Sodium stibogluconate and meglumine antimoniate are of similar efficacy provided their differing concentrations of antimony are taken into account. Only $\mathrm{CL}$ and $\mathrm{DCL}$ due to L.aethiopica are consistently unresponsive (Bryceson 1987). Although there is more extensive data 


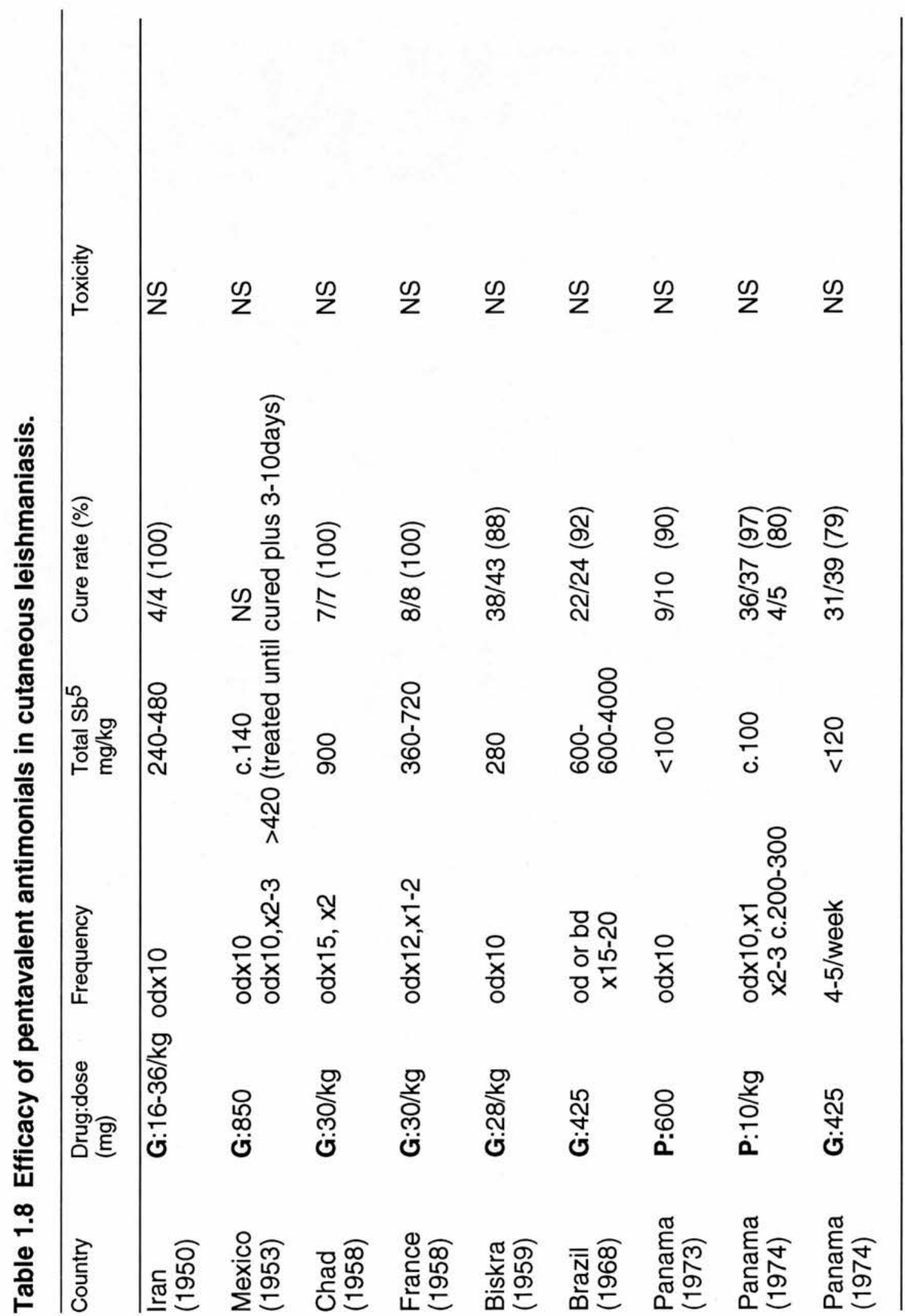




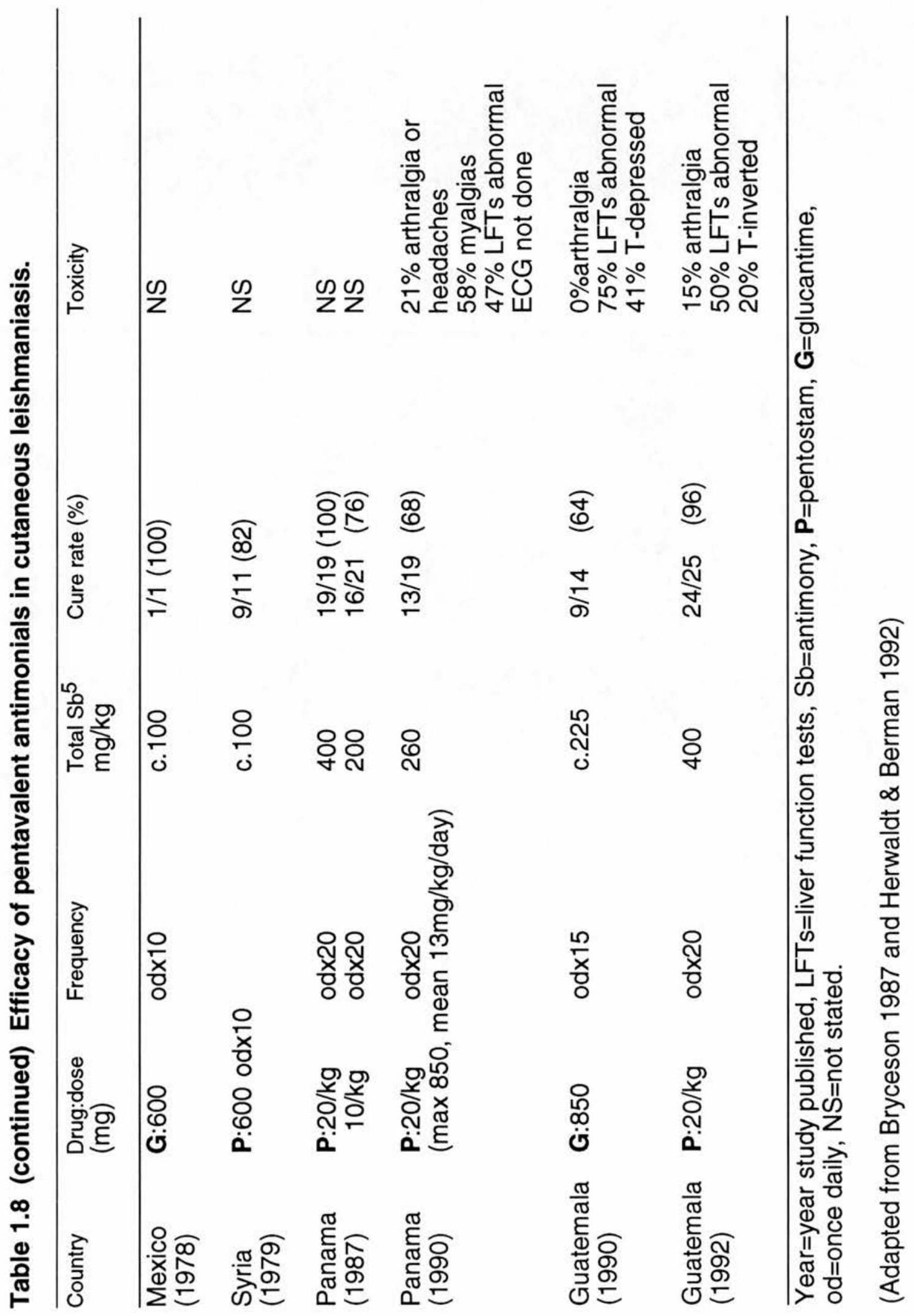


available for its action against visceral leishmaniasis (VL) those results cannot be extrapolated to $C L$ because pentavalent antimonials are preferentially concentrated in the liver and spleen with relatively little reaching the skin. Furthermore, immune responses are less efficient at the cooler temperatures of the skin. This can be illustrated, crudely, by the observation that 40 days treatment cures all patients with VL in Bihar, but 120 days treatment is necessary to cure patients with post kala-azar leishmaniasis (Olliaro \& Bryceson 1993).

Therapeutic regimens. These were developed without access to pharmacokinetic data and with caution resulting from the high toxicity associated with the parent trivalent antimonials. This resulted in the concepts of single daily doses (when bd or tds would be more logical) and of short courses of therapy lasting 6-10 days with repeat courses only after a gap. Indeed, this was still the recommendation in the manufacturer's data sheet for pentostam until recently (Anonymous 1989). This type of regimen has been abandoned by many clinicians in recent years because there is no evidence to support them and, indeed, they may encourage the development of resistant strains (Bryceson 1987). The optimum dosage has not been determined in Old World CL. In New World CL it has been shown that 20 days treatment with sodium stibogluconate $20 \mathrm{mg} / \mathrm{kg} /$ day is more efficacious, and may be no more toxic, than $10 \mathrm{mg} / \mathrm{kg} /$ day for L.panamensis (Ballou et al 1987). The higher-dose regimen will cure virtually all lesions due to L.panamensis in Panama and L.braziliensis in Guatemala (Herwaldt \& Berman 1992). The WHO recommends that when pentavalent antimony is used to treat $\mathrm{CL}$ a dose of 10$20 \mathrm{mg} / \mathrm{kg} /$ day should be given at least until the lesion has healed (WHO 1990). These recent studies indicate that this recommendation should probably be amended in 2 ways. First, $20 \mathrm{mg} / \mathrm{kg} /$ day (sodium stibogluconate) does not appear significantly more toxic, yet it is more effective, than $10 \mathrm{mg} / \mathrm{kg} / \mathrm{day}$. Therefore, unless $10 \mathrm{mg} / \mathrm{kg} /$ day has been reported to be as effective as $20 \mathrm{mg} / \mathrm{kg} /$ day for an endemic region (none so far) CL should be treated with (sodium stibogluconate) $20 \mathrm{mg} / \mathrm{kg} /$ day. Second, both re-epithelialisation of lesions and elimination of parasites continues after antimony therapy has stopped. Treatment until complete clinical and parasitological cure has been achieved therefore constitutes considerable overtreatment. A more logical approach would be to give antimony for a predetermined number of days and 
then examine the patient 4-6 weeks later to see if complete clinical and parasitological cure has occurred. Current data suggests that treatment should last 20 days. If cure rates of $>95 \%$ without relapse are observed for a given area a trial of a lower daily dose or of a shorter duration of antimony treatment could be undertaken (Berman 1988).

The development of antimony resistance has been well documented in visceral leishmanisasis and innate resistance is well recognised for L.aethiopica and, more recently, for L.mexicana (Bryceson 1987, Olliaro \& Bryceson 1993). Parasites causing New World CL and MCL show a wide range of susceptibility to antimonials in vitro. Some isolates from patients unresponsive to antimony are fully resistant and strains of $\mathrm{Lmm}$ and $\mathrm{Lbb}$ from patients who relapse are 10-17 times less susceptible than before treatment. The development of resistance has been linked to discontinuous drug exposure or inadequate dosage. Tests to assess resistance and predict the clinical response to antimony therapy are now under development (Grogl et al 1989, Grogl et al 1992).

Toxicity of the pentavalent antimonials has been, and remains, the subject of intense debate, partly because of the undoubted toxicity of their parent trivalent compounds. Whilst reviewing the available literature Bryceson (1987) concluded that:
'sodium stibogluconate and meglumine antimoniate are amongst the safest drugs in current medical use. They have been used for 40 years, in all races and in most countries in the world, in hundreds of thousands of patients with all forms of leishmaniasis, often malnourished and desperately ill; yet only two deaths have been attributed to their use....... '

However they have a poor reputation because the trivalent antimonials invariably produced cardiac toxicity and reports of ECG abnormalities during sodium stibogluconate therapy appeared relatively early on in the literature (Dempsey 1965, Kaplan et al 1978). A study of 22 British soldiers with CL treated with sodium stibogluconate ( $10 \mathrm{mg} / \mathrm{kg} / \mathrm{day}$ ) failed to find any changes in blood pressure, heart rate, left ventricular function or heart rhythm, although serial ECGs showed a reversible decrease in T-wave amplitude (Henderson \& 
Jolliffe 1985). A study of 65 patients with visceral leishmaniasis treated with sodium stibogluconate (doses ranged from $10-60 \mathrm{mg} / \mathrm{kg} /$ day) serial ECGs showed changes in $54 \%$ of the treatment courses. Furthermore, the proportion of patients with T-wave changes increased with both the daily dose of antimony and the duration of treatment. There was an essentially linear correlation between the proportion of patients with any new ECG abnormality and cumulative dose of antimony administered. The abnormalities observed were T-wave flattening, T-wave inversion and prolongation of the QTc interval - the QTC changes were seen in 13 patients all of which had been treated with more than $20 \mathrm{mg} / \mathrm{kg} /$ day for more than 30 days. These ECG changes were always transient in that they were not present on follow-up. In the few patients who remained in the hospital for initial follow-up, the ECG changes resolved over 13 weeks. Three patients died during treatment: all showed QTc prolongation, ST concavity and T-wave inversion. One patient was presumed to have developed a fatal ventricular arrhythmia and the other two had severe measles infections (Chulay et al 1985). Similar ECG changes have been observed with meglumine antimoniate (Antezana et al 1992). It appears that sodium stibogluconate, in standard doses of up to $20 \mathrm{mg} / \mathrm{kg} /$ day for 20 days, whilst associated with ECG changes is rarely associated with clinical cardiotoxicity (Ballou et al 1987, Dollery 1991) although further studies are needed.

A further cause for concern is the transient increase in liver aminotransferase levels which have been reported during treatment of both visceral (Chulay et al 1983) and cutaneous disease and which may be dose related (Oster et al 1985, Ballou et al 1987). Although these abnormalities settle within 6 weeks of stopping therapy their significance is unknown. A transient fall in the white blood cell count has also been noted during the first few days of therapy but this recovers despite further treatment (Ballou et al 1987). Impairment of renal function during therapy has also been reported ((Viega et al 1983).

Patients frequently complain of loss of appetite, muscle pains and joint stiffness during treatment (table 1.9); these symptoms usually start during the second or third week and may be dose-related. They settle rapidly after the treatment is stopped. Joint effusions can occur during prolonged high dose therapy (Ballou et al 1987, Castro et al 1990). Thrombophlebitis at the site of injection is common. 
Local infiltration of the lesions with either sodium stibogluconate (Kellum 1986) or meglumine antimoniate (Barrios et al 1986, Harms et al 1991) is an alternative to systemic administration for L.tropica, at least, provided there are only a few lesions. However, technique is important and several injections are usually required (WHO 1990).

\section{Aminosidine}

Aminosidine is an aminoglycoside antibiotic produced by Streptomyces chrestomyceticus (Arcamone et al 1959). Its chemical structure is identical is to both paromomycin (which is produced by another streptomyces species) (Schillings \& Schaffner 1961) and monomycin (Reynolds 1993). Like other aminoglycoside antibiotics, aminosidine is poorly absorbed from the gastrointestinal tract. Intramuscular administration leads to prompt and complete absorption with peak levels obtained after one hour of 20 and $40 \mathrm{mg} / \mathrm{L}$ with doses of 0.5 and $1.0 \mathrm{~g}$ respectively. Intravenous administration of the same doses given in saline or $5 \%$ dextrose, over 100 minutes, yields a similar profile. There is relatively little protein binding and it is excreted unchanged through the kidneys. Its pharmacokinetic behaviour fits a multi-compartment model with a dominant elimination half-life of approximately 2.3 hours followed by a slower elimination half-life of about 40 hours (Kahlmetir 1979, Scott et al 1992).

Efficacy. Its anti-leishmanial properties were first reported in the 1960's. Subsequent studies have confirmed these early findings in vitro using hamster peritoneal macrophages (Mattock \& Peters 1975a,b) and in vivo using Balb/c mouse skin lesions (Neal 1968). When compared with sodium stibogluconate it was 6 -times more active against L.mexicana and 600-times more active against L.major. Furthermore it was fully active against antimony resistant strains of L.donovani. It is difficult to interpret in vitro and in vivo experiments, as the assays used and the strain of a given leishmania subspecies affect the result, but it appears that New World cutaneous parasites, particularly Lbb, are less sensitive than Old World and visceral parasites (Neal 1987). There is potentiation between aminosidine and antimonials in vitro against L.mexicana but this effect is less marked in vivo. The mode of action of aminosidine against leishmania is not known (Berman 1991). 


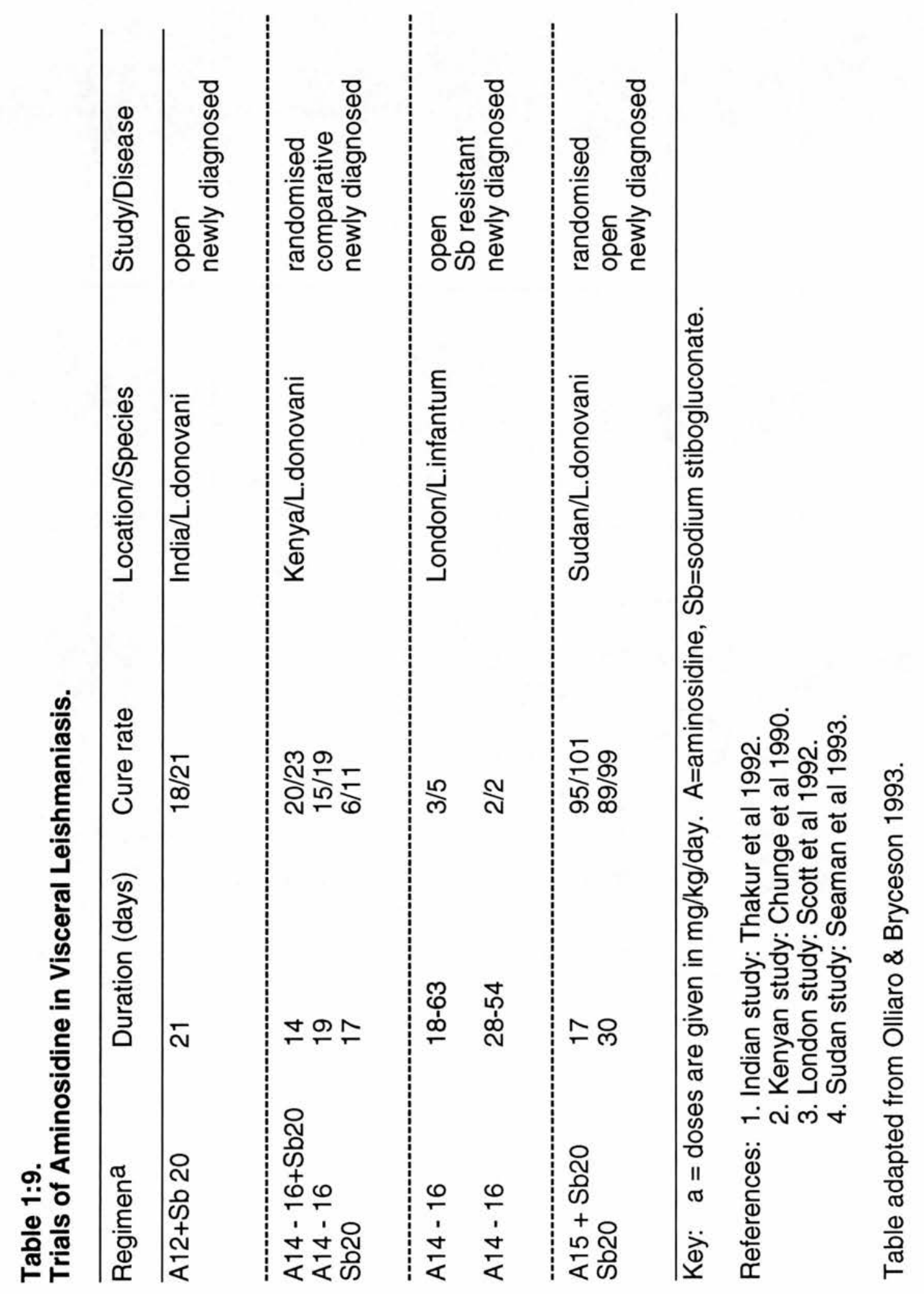


Aminosidine, as monomycin, has been used extensively in Russia to treat CL; when $5000 \mathrm{IU} / \mathrm{kg}$ i.m. was given t.d.s. parasites could not be detected after a mean of 6.9 days and the sores healed in a mean of 13.5 days (Akovbyan \& Mukhamedov 1969, Eršshov 1978). Toxicity was not reported but it may not have been looked for systematically (Bryceson 1987). More recently, aminosidine has been assessed for treating VL in 4 open studies (table 1: 9) which indicate that it may be an effective first-line alternative to pentavalent antimony for the treatment of naive and unresponsive VL. The apparent increase in efficacy of aminosidine when it was combined with sodium stibogluconate noted in the Kenyan study was tested under appalling field conditions in the epidemic of VL in Southern Sudan where it was found that combined therapy could reduce the duration of treatment from 30 to 17 days (Seaman et al 1993). The efficacy of systemic aminosidine for $\mathrm{CL}$ and MCL has yet to be established, although 1 of 2 patients with Old World CL and 1 of 2 with MCL which were resistant to antimony have been successfully treated (Scott et al 1992).

Toxicity. Aminosidine, like all aminoglycosides, is associated with nephrotoxicity and ototoxcity (Ito et al 1968). Toxicity is associated with high plasma concentrations, impaired renal function and concomitant exposure to other nephrotoxic agents (Burkle 1981). Therapeutic doses administered to patients with normal renal and otological function are unlikely to produce toxic reactions, even during prolonged courses of treatment (Donno \& Rimoldi 1974). No clinical or biochemical toxicity was reported in either the Indian or the Kenyan study which is perhaps surprising in view of the well documented elevations in liver enzymes reported during treatment with sodium stibogluconate for $\mathrm{CL}$. The London study was the most closely monitored and patients received higher doses for a longer time; reversible renal impairment occurred in 2 patients and high frequency deafness occurred in one of these patients and another. In the first 3 studies aminosidine was well tolerated and the patients preferred it to the antimonials.

Aminosidine ointment. An ointment containing aminosidine has been pioneered by the group led by Joseph El-On in Jerusalem. Animal studies using Balb/c mice led to the development of a preparation containing $15 \%$ aminosidine and $15 \%$ methyl benzethonium chloride (MBCL) in white soft 
paraffin. Uncontrolled studies in man suggested it would heal lesions due to L.major when applied twice daily for 10 days (El-On et al 1985, El-On et al 1986) and also that it may be effective for lesions due to L.aethiopica (Weinrauch et al 1987) and Lmm (El-On et al 1988). A double blind, placebocontrolled, cross-over trial has recently confirmed its efficacy in L.major infections: $77 \%$ of patients given active treatment were cured within 10 days compared to $27 \%$ in the placebo group (EI-On et al 1992). Although the Israeli group reported inflammation (due to the $\mathrm{MBCL}$ ), other workers have found this to be so severe that treatment could not be continued. When less irritant preparations, containing less $\mathrm{MBCL}$, were used they were less effective (El-Safi et al 1990). A preparation containing urea is currently in Phase 2 to 3 trials in Iran and Tunisia via the WHO. Preliminary evaluation suggests that new formulations may be needed to improve penetration into the lesion (Olliaro \& Bryceson 1993).

In conclusion, treatment of $\mathrm{CL}$ is unsatisfactory. A safe, cheap and either single dose or oral preparation is needed but none is available. The pentavalent antimonials remain the mainstay of therapy but the optimal dose regimen for each geographical area has not been worked out and further assessment of their toxicity is required. Parenteral aminosidine may be effective and safer and, therefore, it should be evaluated in controlled clinical trials. 


\subsection{Cutaneous Leishmaniasis and the British Army in Belize}

\subsubsection{The history and rôle of the British garrison}

Belize is a country the size of Wales lying on the east coast of Central America. It has a population of 150,000 , half of which are under 19 years old. Its main product is sugar. The earliest European settlers were British buccaneers who began arriving in the mid-1 $7^{\text {th }}$ Century making use of the many sheltered anchorages to launch raids on Spanish shipping. Spain mounted repeated attacks against the British settlers but in 1763 granted them rights to fell log wood and eventually recognised British sovereignty in the Treaty of Amiens of 1802. As British Honduras the area had been governed by British administrators from 1786. It became subject to British law in 1840 and was designated a Crown Colony in 1862. The importance of the colony for Britain (which had trading interests all along the Caribbean coast) was primarily economic. It had the best deep-water port facilities in the region, and in the early $19^{\text {th }}$ Century up to $95 \%$ of Central American trade passed through the settlement.

Figure 1.10: Belize.

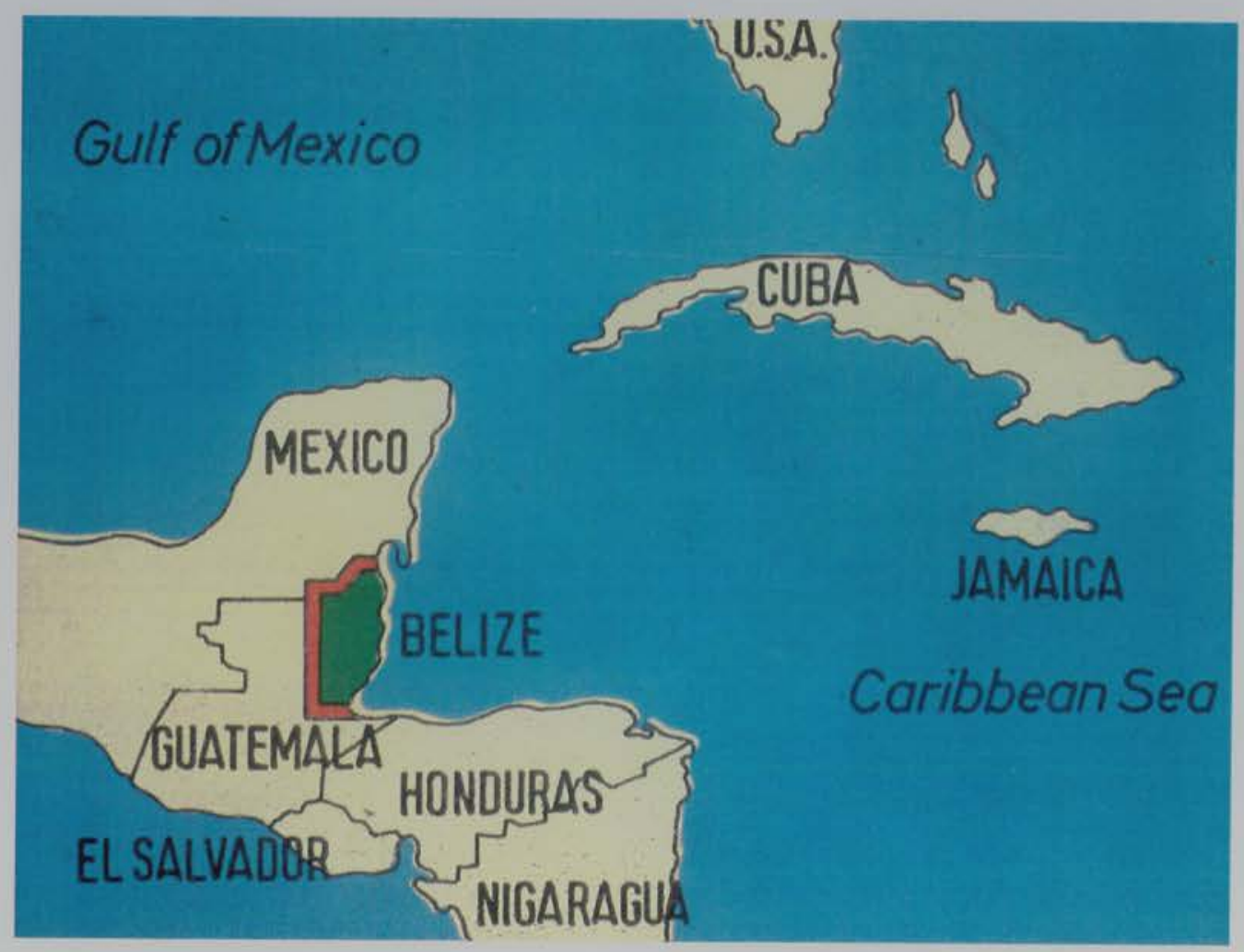


Guatemala gained independence from Spain in 1821 and began to lay claim to Belize (as did Mexico for a time). In 1859 a treaty was concluded between Britain and Guatemala under which Guatemala recognised the colony's boundaries in exchange for a British undertaking to construct a road from Guatemala City to the Caribbean coast. The commercial importance of British Honduras declined markedly with the completion of the Panama railway in 1855, which moved the focus of Central American trade to the Pacific seaboard. Guatemala did not actively pursue Britain's failure to build the road until it abrogated the treaty in 1945 , claiming that Britain had failed to fulfil its obligations (Brooks 1983, Goetz 1991).

The colony was granted internal self-government in 1964, it was renamed Belize in 1973, and granted full independence on $21^{\text {st }}$ September 1981. As a result of the moves towards independence Guatemala renewed its claim to the territory. Since then Anglo-Guatemalan talks on this issue have repeatedly broken down. At the time of independence Belize had a very rudimentary army - the Belizian Defence Force numbering only 500 soldiers; consequently the British government agreed to provide a defensive garrison to deter the large Guatemalan forces across the border. The formal mission of the British Forces is four-fold: (1) to provide assistance to the Belize Defence force in the defence of Belize against any external aggression, (2) to assist the Belizian Defence Force with training, (3) to keep track of what is going on in the area, (4) to provide assistance to the civil power in disaster relief and in the maintenance of emergency services. The garrison consists of approximately 1800 troops and airmen, mostly on 6 month, unaccompanied tours of duty, from many different parts of the Army and the Royal Air Force; many undertake jungle training during their tour (Fursden 1988).

\subsubsection{The cutaneous leishmaniasis problem}

The deployment of troops to Belize in the late 1970's in preparation for independence was soon followed by the appearance of cases of $\mathrm{CL}$; from 1977-1990 a total of 319 soldiers contracted it during or immediately after their tour of duty (figure 1.1i). The US army has had a similar experience in Panama 
(Takafuji et al 1980); from 1977-1984 the Walter Reed Medical Center in Washington DC treated 70 cases from that theatre (Ballou et al 1987). This is not surprising. As described earlier, New World leishmaniasis is essentially a zoonosis maintained by forest rodents. Most cases are occupational and are related to the length of time the non-immune worker spends in the forest in contact with potentially infected sandflies. The incidence is very variable and this is probably related to the presence of "hot-spots" - some units are unfortunate enough to select one of these areas for training.

Figure 1.11: Incidence of CL in British troops from Belize

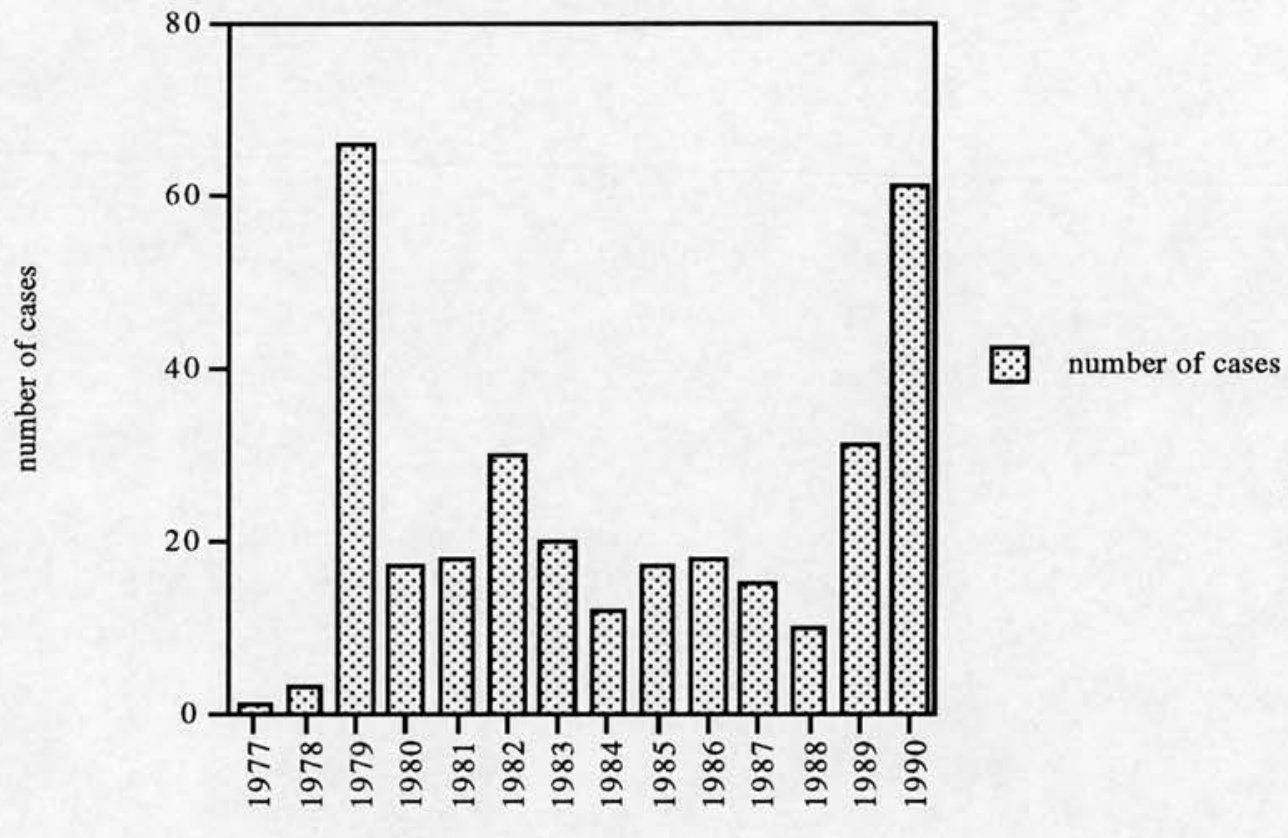

Year

The first cases of CL caused some difficulties in diagnosis and it took time to develop expertise in diagnosis and management. The large number of cases in 1979 (coupled with a headline in the Sun newspaper: 'British Troops Beaten Back By Jungle Flies') prompted a concerted public health programme within the Army but also involving the RAF and the Royal Navy. All suspected cases were sent back to the Dermatology Department of the Cambridge Military Hospital in Aldershot where expertise was concentrated. Soldiers and their regimental medical officers were briefed about $\mathrm{CL}$ prior to departure and they 
were issued with a 'Leishmaniasis Warning Card' upon their return to show to any medical attendant consulted within 2 years. This resembled a credit card and recommended referral to Aldershot if CL was considered a possibility. Preventative measures such as impregnation of mosquito nets with permethrin were also introduced.

However, the very nature of their task means that the troops continue to be bitten and CL has become a major health problem for the British Army. This is likely to continue as long as troops remain in Belize.

Figure 1.12: British troops training in Belize.

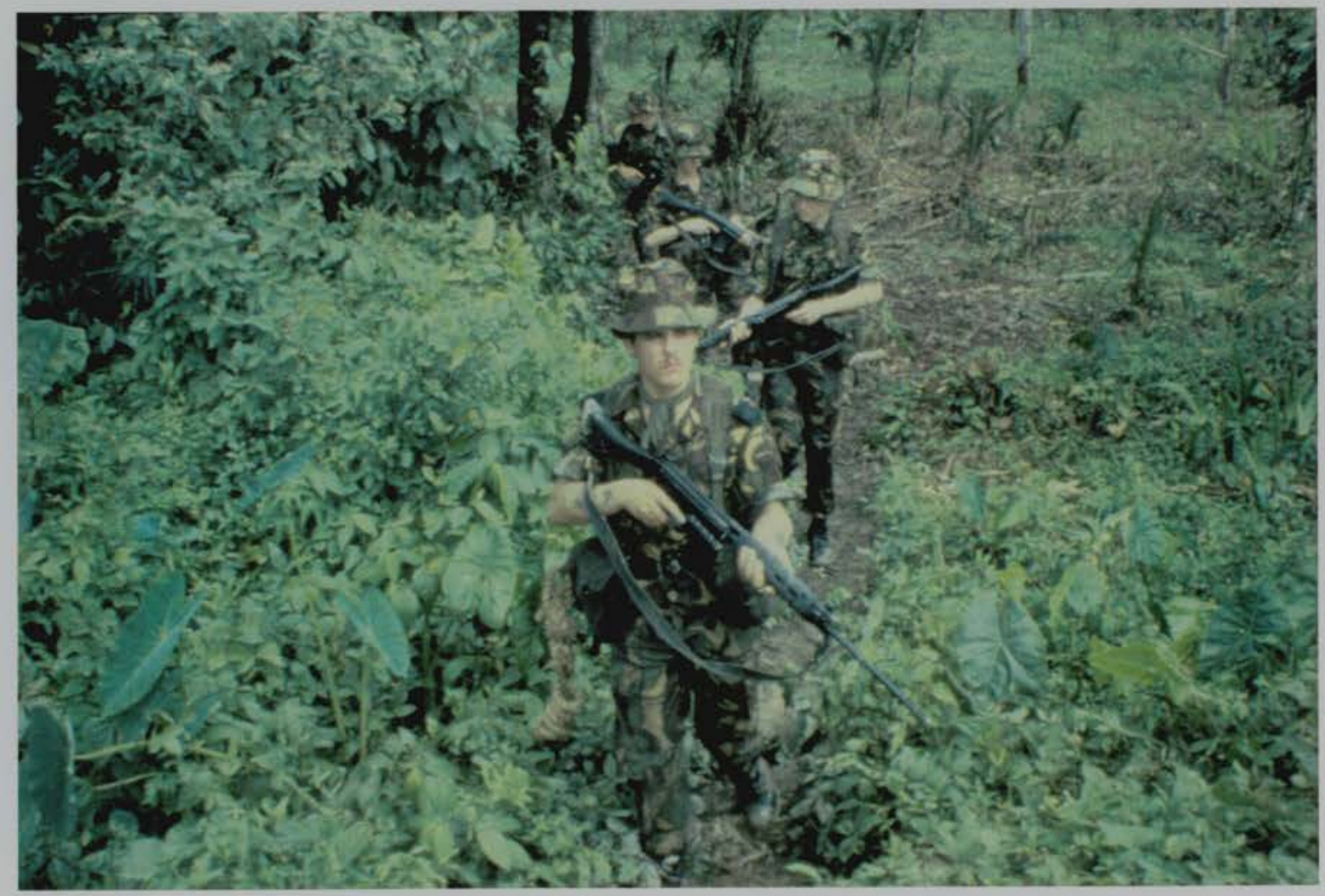




\section{Chapter 2}

\section{A Retrospective Study of Belizian Cutaneous Leishmaniasis in British Troops From 1978 to mid- 1990.}

2.1 Aims of the investigation

2.2 Introduction

2.3 Methods

2.4 Results

2.5 Discussion

2.6 Summary 


\subsection{Aims of the Investigation}

To determine the clinical features of cutaneous leishmaniasis in British troops returning from Belize.

To determine the diagnostic criteria used.

To determine the relative frequency of cases caused by $L \mathrm{~mm}$ and $L b b$.

To identify any clinical differences between lesions due to $\mathrm{Lmm}$ and those due to Lbb.

To identify the types, efficacy and toxicity of therapies used.

\subsection{Introduction}

In the 12 years from 1978 until mid-1990 over 300 soldiers were treated for CL in the Dermatology Department of the Cambridge Military Hospital, Aldershot, the central referral hospital for all cases of $\mathrm{CL}$ in the British Armed Forces. The data from this large series of patients had never been systematically analysed. Before any further studies could be contemplated, it was, therefore, necessary to determine which of the many clinical impressions formed over the years could be substantiated, thereby defining our knowledge of Belizian CL and its management.

As knowledge of $\mathrm{CL}$ increased so management changed. The most important discovery was the isolation of Lbb, with its potential complication of espundia, in some of the returning soldiers (Evans et al 1984). Until that time all cases of Belizian $\mathrm{CL}$ had been assumed to be due to $\mathrm{Lmm}$ following the classical work by Lainson and Strangeways-Dixon (1963). Lesions due to Lmm and Lbb were thought to be clinically identical. Numerous therapeutic modalities had been used on an ad-hoc basis but sodium stibogluconate had been found to be the most useful. Initially short, low-dose courses were used but later longer courses at a higher daily dose had been adopted and found more efficacious. 


\subsection{Methods}

A clinical diagnosis of CL had been made in 306 servicemen between 1978 and mid-1990 following a tour of duty in Belize. Their hospital case notes were scrutinised and the following data extracted: age; dates of Belize tour; date lesion first noticed; number, size and site of the lesions; the results of histological examination of the lesions; culture and isoenzyme typing of the parasite. Detailed records were made of the treatment given including dates, drug, dose, duration, side-effects, and the haematological and biochemical parameters used in monitoring treatment. Resolution of the ulcers, an objective end point that could be assesssed easily and reliably by all physicians, was the standard criterion for cure. Patients were closely supervised by army dermatologists to ensure that cure was attained and maintained for at least 3 months. Thereafter patients were usually followed-up by their regimental medical officer and re-referred if they relapsed.

For the purposes of this thesis, detailed analysis was restricted to the 187 cases in which the clinical diagnosis was confirmed by demonstration of the parasite by culture and/or histological identification of Leishman-Donovan (LD) bodies. In general a single lesion had been sampled and other, clinically similar, lesions were assumed to be of the same aetiology. A comparison of the clinical features was made between those cases in which Lmm and those in which Lbb had been identified.

During the early part of the 12 year period covered by this study the main treatment strategy consisted of an initial 10 day course of intravenous (iv) sodium stibogluconate (Pentostam ${ }^{R}$ ) $600-800 \mathrm{mg}$ daily. If the ulcers were not healing satisfactorily this was either continued, or repeated after a break, as recommended by the manufacturer (Anonymous 1989). Because many patients required repeated courses a higher dose regimen was subsequently adopted. This consisted of an initial 14 day course of iv sodium stibogluconate $600 \mathrm{mg}$ twice daily. Again, if the ulcers were not healing satisfactorily, this was either continued or repeated after a break. The success of these strategies was compared. Haematological and biochemical monitoring parameters prior to treatment, at the end of treatment, and 2 weeks after cessation of treatment, were available for 2 cohorts, one treated with $600 \mathrm{mg}$ daily iv for 10 days to a total of $6 \mathrm{~g}$ (36 cases) and 
a second treated with $600 \mathrm{mg}$ twice daily iv for 14 days to a total of $16.8 \mathrm{~g} \mathrm{(24}$ cases).

Statistical analysis. Pre-treatment lesion size and duration in all the cases, and in the Lmm and Lbb subgroups, were statistically evaluated using an unpaired ttest. Differences in the haematological and biochemical parameters before, during and after treatment, were evaluated using a paired t-test.

\subsection{Results}

A clinical diagnosis of $\mathrm{CL}$ was made in $\mathbf{3 0 6}$ otherwise healthy male soldiers, mean age 23 years (sd 3.9), who had acquired their infection whilst on active service in Belize. Confirmation of the diagnosis by demonstration of the parasite was made in 187 cases (61\%): a positive culture was obtained in 117 cases, and LD bodies were identified in a further 70 cases. Of the 117 culture-positive cases the species was identified in 107: 77 were due to Lbb, 29 to Lmm, and in one case both Lbb and $\mathrm{Lmm}$ were identified from a single lesion (figure $2: 1$ ). Lesions started as small inflammatory papules which gradually enlarged and ulcerated. The ulcer was often covered with an adherent crust (figure 2.2) which subsequently separated to expose a necrotic ulcer base which was frequently secondarily infected with either staphylococci aureus or ß-haemolytic streptococci. The fully developed lesion consisted of a painless ulcer with a necrotic base and an indurated violaceous margin (figure 2.3).

A total of 283 lesions were identified in the 187 parasitologically confirmed cases: a solitary lesion was present in $71 \%$, two lesions in $16 \%$, three lesions in $8 \%$ and four lesions in $4 \%$. One soldier had five lesions and another had 12 lesions.

Cases due to $\mathrm{Lmm}$ and $\mathrm{Lbb}$ had similar numbers of lesions and most occurred on exposed body sites with the head, neck, wrists and hands accounting for $51 \%$ of lesions. Lesions due to $\mathrm{Lmm}$ were more common on the head and neck than those due to Lbb (figure 2.4). The mean diameter (longest axis) of all ulcers was 14.4 $\mathrm{mm}$ (sd 9.3), lesions on the head and neck being smaller than those elsewhere at presentation. The lesions due to Lbb were significantly $(p<0.01)$ larger (mean diameter $17 \mathrm{~mm}$ ) than those due to $\mathrm{Lmm}$ (mean diameter $12.3 \mathrm{~mm}$ ), despite the observation that Lbb lesions had been present for a significantly $(p<0.05)$ shorter 


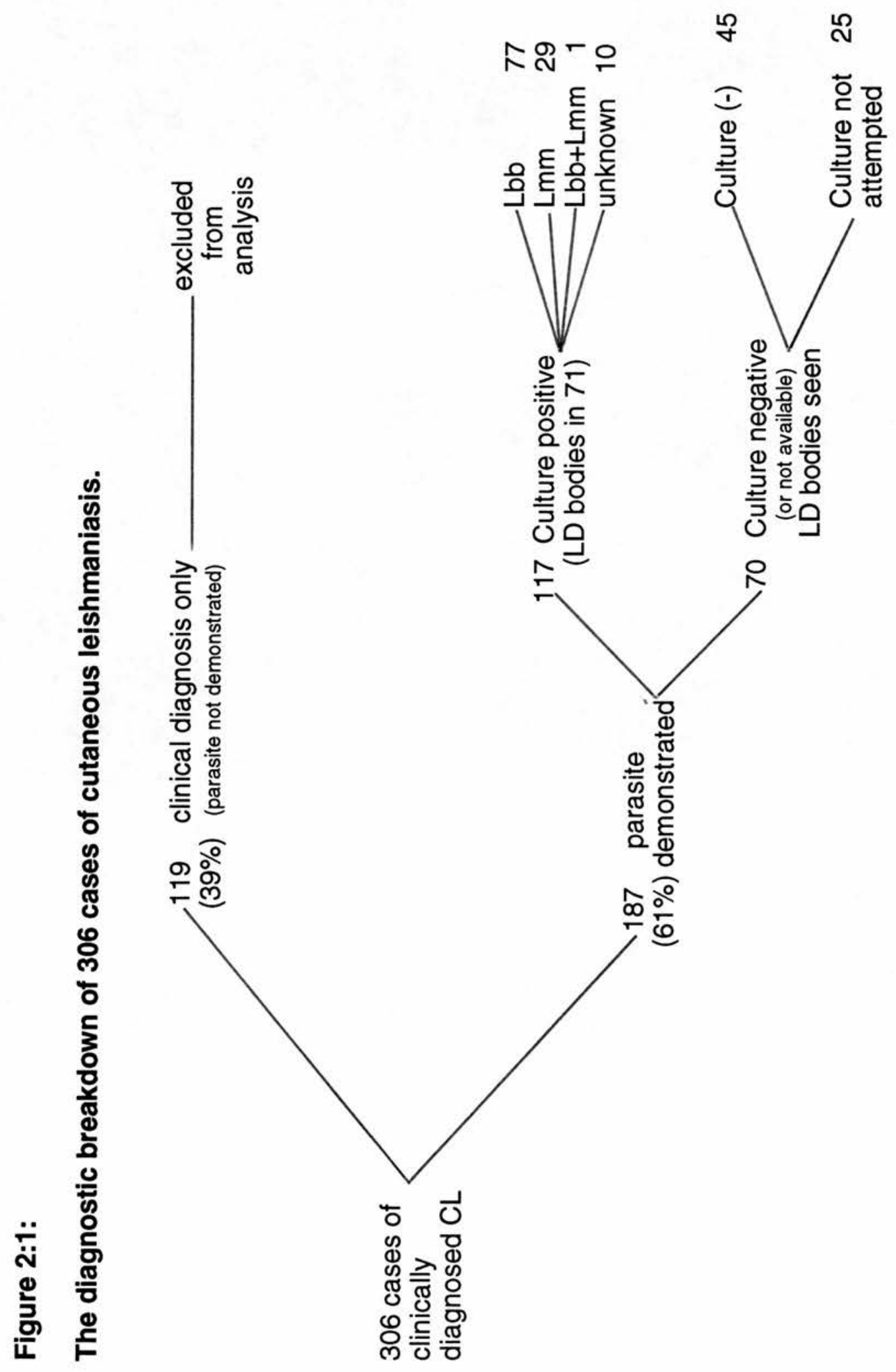


Figure 2.2: Early lesion of cutaneous leishmaniasis from Belize with adherent crust.

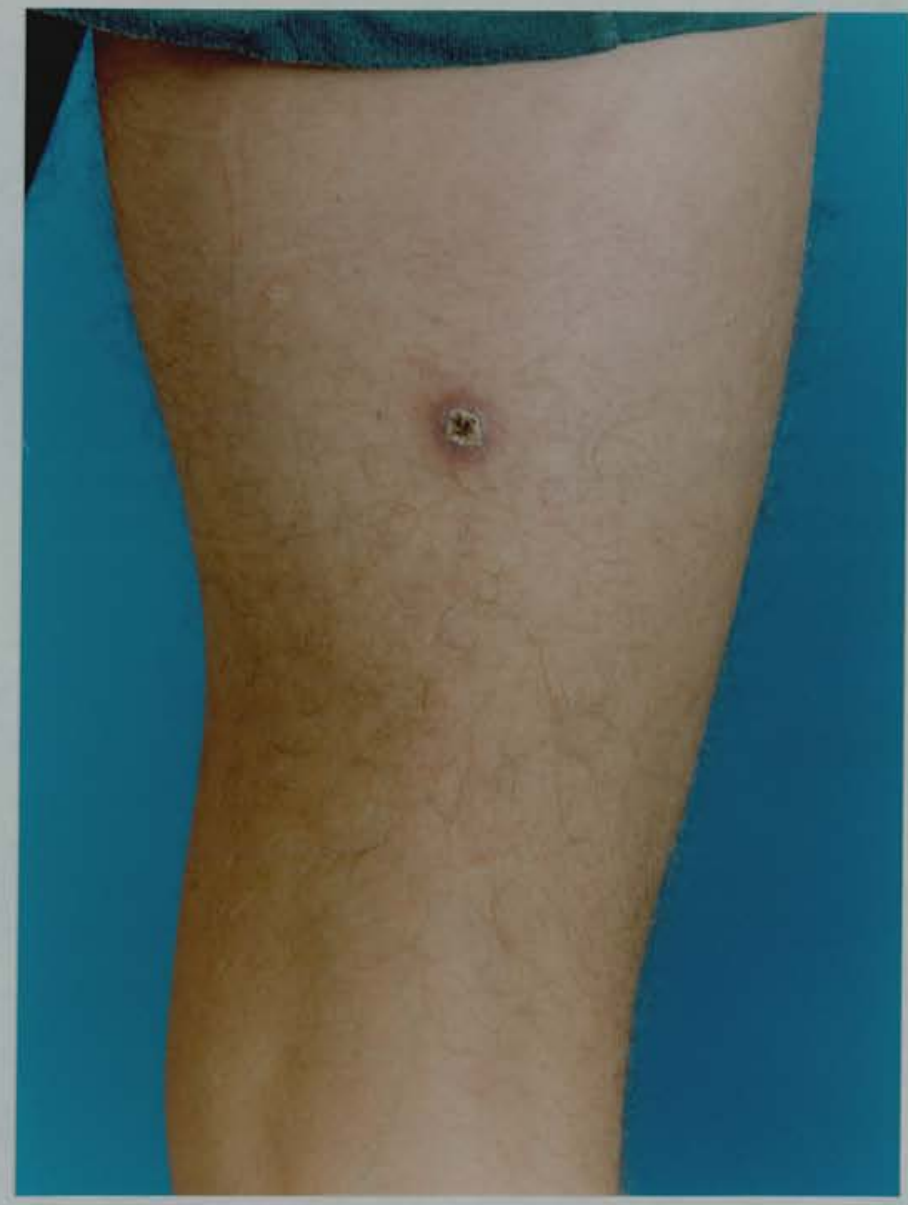

Figure 2.3: Later lesion in which crust has separated to form an ulcer.

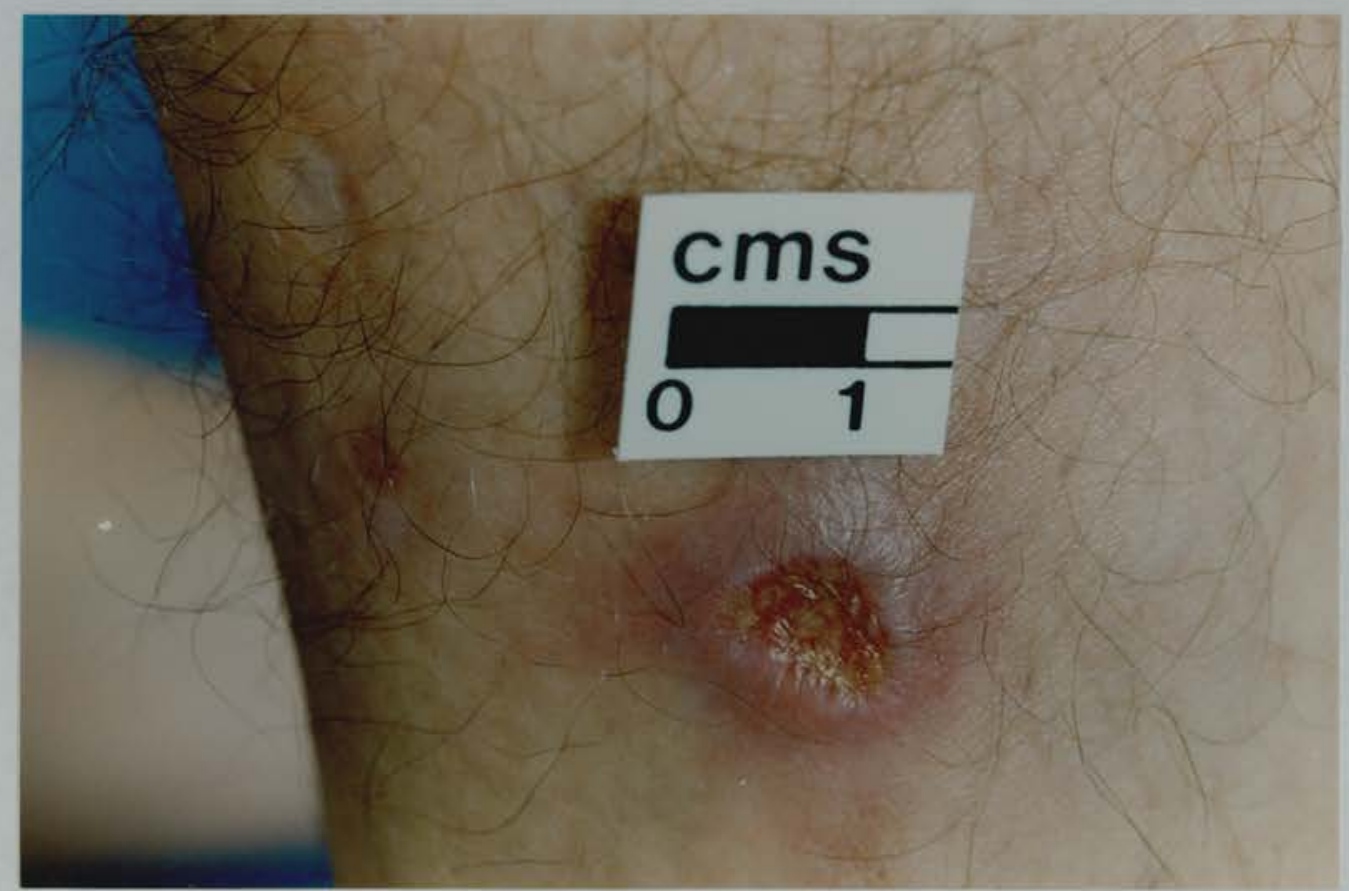


Figure 2.4: Site of ulcers in cases due to Lmm (top) and Lbb (bottom) - Each spot represents $1 \%$ of lesions.
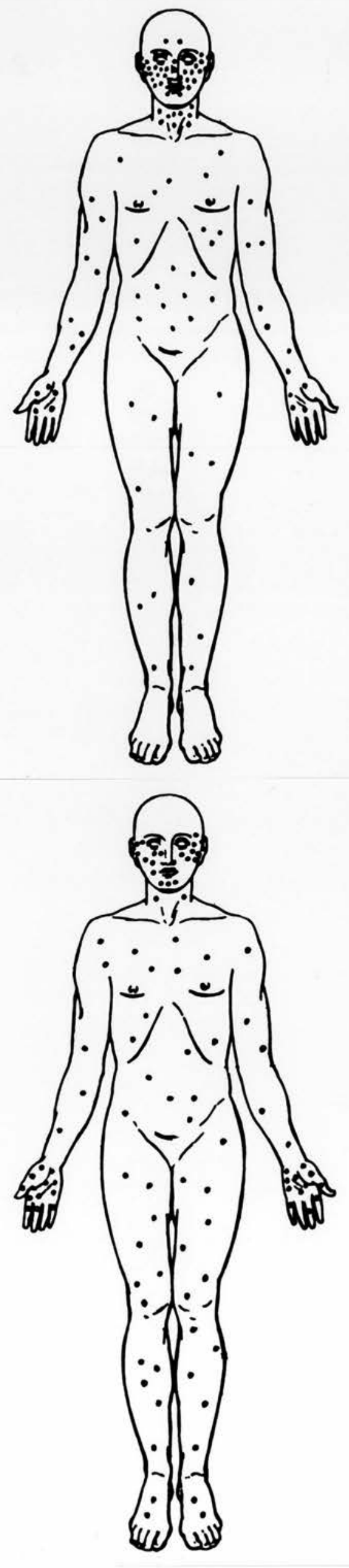
time (mean 8.8 weeks) than Lmm lesions (mean 12.5 weeks). Most soldiers presented either during their Belize tour of duty, or within 4 months of their return. One soldier presented 11 months after his return. The data for these analyses are shown in appendix 1.1. These clinical parameters were very similar in the 187 parasitologically proven cases to the whole group of 306 cases in which a clinical diagnosis of CL had been made (appendix 1.2).

Treatment started after a mean interval of 9.9 weeks (sd 6.7) had elapsed after the lesion was first noticed. Sodium stibogluconate was the mainstay of treatment. The ulcers of 166 soldiers healed with sodium stibogluconate alone, although there was a wide range in the total dose required (mean $13.1 \mathrm{~g}$, sd 7.2). During the early part of the study 101 patients were treated with a low $(600-800 \mathrm{mg})$ daily dose regime. Clinical side-effects were recorded in only one case - that soldier reported aching muscles after receiving $800 \mathrm{mg}$ daily for 11 days. The higher dose regime (600 mg twice daily) which was subsequently adopted was associated with greater morbidity; of the 72 soldiers who received this regime 3 became nauseated and anorexic, 11 developed myalgia, 2 developed chest pain (which was associated with tender costal cartilages), 2 reported non-specific malaise and 2 developed thrombophlebitis at the site of the indwelling iv cannula, associated with pyrexia. One soldier developed cheiropompholyx, although that resolved with appropriate treatment whilst sodium stibogluconate was continued. One soldier developed an acute aseptic arthritis of his knee 2 days after completing a course of $16.8 \mathrm{~g}$ and another developed severe hip pain, accompanied by a serum creatine kinase level of $25,290 \mathrm{lU} / \mathrm{l}, 14$ days after receiving $23.4 \mathrm{~g}$ sodium stibogluconate. Both problems gradually resolved over the following 4 months without specific therapy.

Sequential haematological and biochemical monitoring parameters, assessed prior to treatment, at the end of treatment and 2 weeks after treatment, were available for 2 cohorts. Although laboratory data were available for other soldiers they had not been collected systematically and, therefore, their analyses could have been misleading. The first cohort received the 'standard' low-dose regimen consisting of $600 \mathrm{mg} /$ day to a total of $6 \mathrm{~g}$ ( 36 soldiers) and the other received the 'standard' highdose regimen consisting of $600 \mathrm{mg}$ twice daily to a total of $16.8 \mathrm{~g}$ ( 24 soldiers). A mild, although significant $(P<0.05)$, leukopenia was noted at the end of treatment in both groups, but there was no change in the haemoglobin level. The serum urea did not rise during treatment. Serum aminotransferase levels (alanine 
aminotransferase (ALT) and aspartate aminotransferase (AST)) were both elevated at the end of treatment but had almost returned to pre-treatment levels 2 weeks later. These changes were more marked in the group which received the high-dose regimen. The serum glutamyl transferase (GGT) was only elevated in the high-dose group at the end of treatment and it had also returned to normal 2 weeks later. The alkaline phosphatase (ALK P) level was elevated in the highdose group at the end of treatment and in both groups 2 weeks later. A transient fall in serum creatine kinase at the end of treatment was noted in both groups. There was no significant rise in lactate dehydrogenase. These changes are summarised in table 2.1 and the detailed results are given in appendix 1.3. The clinical features of the soldiers given the low and high dose regimens are shown in table 2.2.

\section{Table 2:1}

Haematological and biochemical changes during treatment with sodium stibogluconate.

White cell count

Alkaline phosphatase

Gamma glutamyl transferase

Alanine aminotransferase

Aspartate aminotransferase

Creatine kinase

\section{Low-dose regimen}

White cell count

Alkaline phosphatase

Gamma glutamyl transferase

Alanine aminotransferase

Aspartate aminotransferase

Creatine kinase

$\begin{array}{lll}\text { Pre-Rx } & \text { Post-Rx } & \text { Recovery } \\ N & \downarrow & N \\ N & N & \uparrow \\ N & N & N \\ N & \uparrow & \uparrow \\ N & \uparrow & N \\ N & \downarrow & N\end{array}$

High-dose regimen

$\begin{array}{lll}\text { Pre-Rx } & \text { Post-Rx } & \text { Recovery } \\ N & \downarrow & N \\ N & \uparrow & \uparrow \\ N & \uparrow & \uparrow \\ N & \uparrow & \uparrow \\ N & \uparrow & \uparrow \\ N & \downarrow & N\end{array}$

Changes are indicated at the end of treatment and 2 weeks after treatment stopped (recovery).

$\mathrm{N}=$ within normal range, arrows indicate significant variation $(\mathrm{P}<0.05)$ from the pretreatment value. 
Table 2.2

Clinical Features of Soldiers Allocated to Low and High Dose Regimens

\begin{tabular}{|c|c|c|}
\hline & $\begin{array}{l}\text { Low-dose } \\
\text { regimen }\end{array}$ & $\begin{array}{l}\text { High-dose } \\
\text { regimen }\end{array}$ \\
\hline Number of patients & 101 & 72 \\
\hline Mean number of lesions & 1.54 & 1.47 \\
\hline $\begin{array}{c}\text { Mean duration of lesions prior } \\
\text { to treatment (weeks) }\end{array}$ & $9.2(\mathrm{sd} 6.9)$ & $8.8(\mathrm{sd} 4.4)$ \\
\hline \multicolumn{3}{|l|}{ Mean lesion size } \\
\hline (longest axis of ulcer, $\mathrm{mm}$ ) & $15.9^{\star}(\mathrm{sd} 9.9)$ & $12.39(\mathrm{sd} 7.7)$ \\
\hline \multicolumn{3}{|l|}{ Lesion site } \\
\hline Head and neck & $39(25 \%)$ & $28(26 \%)$ \\
\hline Trunk & $26(17 \%)$ & $16(15 \%)$ \\
\hline Arms & $16(10 \%)$ & $14(13 \%)$ \\
\hline Hands/wrists & $42(27 \%)$ & $25(24 \%)$ \\
\hline Legs & $31(20 \%)$ & $20(19 \%)$ \\
\hline Not recorded & $2(1 \%)$ & $3(3 \%)$ \\
\hline \multicolumn{3}{|l|}{ Species typing } \\
\hline Lmm & 18 & 5 \\
\hline Lbb & 41 & 31 \\
\hline $\mathrm{Lmm}$ and $\mathrm{Lbb}$ & 0 & 1 \\
\hline Not available & 42 & 35 \\
\hline
\end{tabular}

* significant difference $P<0.05$ 
The low daily dose sodium stibogluconate regime $(600-800 \mathrm{mg}$ daily) was initiated in 101 soldiers. Treatment was given for 10 days in the first instance after which it was either continued or repeated after a break. This initial 10 day course cured 49 $(49 \%)$ of those soldiers and, ultimately, the ulcers of $94(93 \%)$ healed on this regimen. In the remaining 7 cases treatment with sodium stibogluconate was stopped and alternative treatments given because of dissatisfaction with their clinical progress. The ulcers of $90 \%$ of soldiers treated with this low-dose regimen had healed after a cumulative dose of $24 \mathrm{~g}$ (ie 40 days treatment).

The high daily dose sodium stibogluconate regimen, (600 mg twice daily) was initiated in 72 soldiers. Treatment was given for 14 days in the first instance, after which it was either continued, or repeated after a break. The initial 14 day course cured $46(63.9 \%)$ of these soldiers, and ultimately the ulcers of all these soldiers healed with this regimen. The ulcers of $90 \%$ of soldiers treated with this regimen had healed after a cumulative dose of $24 \mathrm{~g}$ sodium stibogluconate (ie 20 days treatment).

Fourteen soldiers were initially given treatments other than sodium stibogluconate. Seven were treated with ketoconazole $800 \mathrm{mg}$ daily for 28 days following which the ulcers of 5 had healed - the remaining 2 healed after a course of $7 \mathrm{~g}$ sodium stibogluconate. Three soldiers, all with infections due to Lbb, were given a one month course of rifampicin $150 \mathrm{mg}$ combined with isoniazid $150 \mathrm{mg}$ twice daily; one healed but the other 2 required further treatment with $7 \mathrm{~g}$ sodium stibogluconate. Three soldiers with ulcers caused by Lmm were treated by local excision. One soldier with an ulcer due to Lbb was successfully treated with pentamidine $4 \mathrm{mg} / \mathrm{kg}$ iv on alternate days for 3 weeks; pentamidine had been chosen because during treatment for $\mathrm{CL}$, type unknown, with sodium stibogluconate several years earlier he had developed an exfoliative dermatitis; when he was subsequently rechallenged with a small dose of sodium stibogluconate he developed cheiropompholyx.

As described above 7 soldiers ( 6 due to $\mathrm{Lmm}$ and 1 culture negative) in whom the low-dose regimen had been started were subsequently given alternative treatment because of dissatisfaction with their progress. Sodium stibogluconate was stopped after various doses: $6 \mathrm{~g}, 12 \mathrm{~g}, 12 \mathrm{~g}, 12 \mathrm{~g}, 18 \mathrm{~g}, 20 \mathrm{~g}$ and $21.5 \mathrm{~g}$. The alternative 
treatments included pentamidine, ketoconazole, itraconazole and paromomycin ointment. All ultimately healed.

The lesions recurred in 7 soldiers following initially successful treatment. In 6 soldiers this occurred between 4 and 7 months after healing and in the remaining soldier after 19 months. Six were successfully re-treated with sodium stibogluconate and in the remaining case the lesion was excised. Mucocutaneous leishmaniasis has not, as yet, developed in any British soldier following service in Belize.

\subsection{Discussion}

There are few reports which document the clinical features of New World CL, the largest is that of Llanos-Cuentas in which the lesions of 182 cases with active disease (predominantly due to $\mathrm{Lbb}$ ) are described (Lainson \& Strangeways-Dixon 1963, Llan os-Cuentas et al 1984, Ballou et al 1987, Jones et al 1987, Walton 1987). One further problem is that interpretation of data from many of these studies is difficult because the diagnosis has not been confirmed by demonstration of the parasite in all cases. In this study, although a clinical diagnosis was made in 306 cases, analysis was restricted to the 187 cases in which the parasite was demonstrated. In each case a single lesion had been biopsied and it was assumed that other, clinically similar, lesions in the same soldier were of the same aetiology. Seventy-one percent of cases presented with a solitary lesion, usually on the exposed extremities, which is similar to other reports. However, in this study a greater proportion of lesions occurred on the hands and wrists compared with the legs. This may be attributable to the fact that natives work in the jungle with bare legs whereas soldiers wear boots and long trousers. A higher proportion of lesions due to Lmm occurred on the head and neck. This has been attributed to the biting habits of Lutzomyia olmeca olmeca, the vector of Lmm, which tends to bite at head level (Walton 1987). The vector of Lbb in Belize has not yet been established. Interestingly, this predominance of head and neck lesions was also noted 20 years ago by Lainson and Strangeways-Dixon (1963), at which time all lesions in Belize were thought to be caused by $\mathrm{Lmm}$. The average diameter of lesions at presentation was smaller than reported in other studies; this is probably the result of earlier presentation. 
Diagnosis may be difficult. In the early stages the lesion resembles an insect bite and later, the presence of secondary infection, may lead to further diagnostic difficulty. Demonstration of the parasite is crucial to confirm the diagnosis. Histological identification of the parasite by staining with Giemsa, and culture, followed by isoenzyme typing, were the main methods employed. However, parasitological confirmation was only obtained in $61 \%$ of these cases. There is a need for more sensitive diagnostic tests - at present most centres rely on a battery of different diagnostic methods including histology, culture, impression smears and serology (Berger et al 1987, Manson-Bahr 1987).

Lbb was isolated from 77 cases, $\mathrm{Lmm}$ from a further 29 , and in one additional case both $\mathrm{Lbb}$ and $\mathrm{Lmm}$ were identified from a single lesion. As it is more difficult to culture Lbb compared with Lmm (which grows readily) (Manson-Bahr 1987), it is likely that $\mathrm{Lbb}$ is the most prevalent of $\mathrm{CL}$ which requires treatment in Belize. It is therefore imperative that all such cases receive effective systemic therapy to prevent the late development of espundia (Bryceson 1987). The identification of Lmm from a single lesion would not appear to remove this obligation. Lesions due to $L b b$ and $L m m$ cannot be differentiated by their clinical features, although there is an indication that those due to Lmm grow more slowly.

This study confirms the relative safety and efficacy of sodium stibogluconate which has an unjustifiably poor reputation because of the toxicity of the earlier trivalent antimonials (Bryceson 1987). The manufacturer of sodium stibogluconate (Pentostam ${ }^{R}$ ) has, until recently, recommended a 10 day course of $600 \mathrm{mg}$ iv daily which may be repeated after a 10 day rest (Anonymous 1989). A single such course cured $48.5 \%$ of our cases, with minimal side effects. A higher dose regimen was subsequently introduced because of dissatisfaction with the high proportion of soldiers requiring further courses of treatment, coupled with increased confidence in the safety profile of the drug. This regimen consisted of an initial 14 day course of $600 \mathrm{mg}$ iv twice daily which was either continued, or repeated after a break, if the ulcers were not healing satisfactorily. The initial 14 day course cured $63.9 \%$ of cases, and all those treated with this regimen healed with sodium stibogluconate alone. It was notable that in both the low and high-dose regimens $90 \%$ of cases were cured when $24 \mathrm{~g}$ of sodium stibogluconate had been administered. This supports the principle that the efficacy of antimonials depends 
upon the total dose that can be administered within a given period of time (Bryceson 1987). It is not possible to determine whether the 7 soldiers withdrawn from the low dose regimen would have ultimately healed if sodium stibogluconate had been continued, or whether there was true resistance to antimony at that dose. It may be significant that 6 of these 7 soldiers had lesions due to $\mathrm{Lmm}$. The development of antimony resistance is a cause for concern and it has been linked to inadequate initial daily doses and treatment duration (Bryceson 1987).

The high-dose regimen was associated with greater morbidity, particularly arthralgia; this has been observed by others (Marsden et al 1985). The fall in white blood cell count and rise in aminotransferase and alkaline phosphatase levels, which are objective evidence of toxicity, although a feature of both regimens, were of greater magnitude in those who had received the higher daily dose. Higher dose regimens have been developed by others (Ballou et al 1987, Oster et al 1985 ) but significant dose-dependant toxicity has not been reported. There was considerable individual variation in these biochemical and haematological changes, which usually developed in the absence of symptoms, and which were reversible after treatment stopped. It is unlikely, however, that factors other than the therapy caused these changes because patients were confined to hospital during treatment. It was reassuring that the serum urea did not rise during treatment as renal tubular dysfunction has been reported during therapy (Veiga et al 1983). The only cardiac effect noted in a group of 22 of these soldiers who had received the low-dose regimen was a reversible flattening of the T-wave (Henderson \& Jolliffe 1985). Data was not available to study the cardiac effects of the high-dose regimen but these effects have been reported to be dose-related by others studying sodium stibogluconate therapy in visceral leishmaniasis (Chulay et al 1985).

These results indicate that sodium stibogluconate $600 \mathrm{mg}$ iv twice daily for 14 days, with an option to extend or repeat the course if necessary, is an appropriate regimen for the treatment of Belizian $\mathrm{CL}$ and it can be expected to cure all cases. The initial 14-day course cured $63.9 \%$ of these cases and, if extended to 20 days a cure rate of $90 \%$ would be anticipated. The higher dose regimen is associated with greater morbidity, but the development of drug resistance is less likely to become a clinical problem if higher dose regimens are given in the first instance for a 
reasonable period of time. Other types of treatment have been tried on an ad-hoc basis, but none has been found as useful and none formally evaluated.

All these soldiers were followed up for at least 3 months by army dermatologists after which follow up was continued by their regimental medical officer. It is unlikely that relapses would have gone unnoticed. No cases of mucocutaneous leishmaniasis have occurred. In the light of the pattern of relapses observed it would seem appropriate to keep patients with Belizian CL under review for at least 2 years. 
2.6 Summary: A retrospective study of cutaneous leishmaniasis in British troops from Belize from 1978 to mid-1990

1. The medical records of 306 British soldiers in whom a clinical diagnosis of $\mathrm{CL}$ had been made following a tour of duty in Belize were analysed. The diagnosis had been confirmed by demonstration of the parasite in $187(61 \%)$ of cases; leishmania were cultured in 117 cases and L-D bodies were identified in a further 70 cases. In the remaining cases only a clinical diagnosis had been made and, although the clinical features of this group did not differ significantly from those in which the parasite was demonstrated, these cases were eliminated from further analysis.

2. Lbb was identified by isoenzyme analysis in 78 cases and Lmm in a further 29 cases. In one case both $\mathrm{Lbb}$ and $\mathrm{Lmm}$ were identified from a single lesion.

3. Seventy-one percent of patients had a single lesion which usually occurred on the exposed extremities. Lesions due to Lmm were more common on the head and neck.

4. The mean diameter of the ulcers was $14.4 \mathrm{~mm}$ and lesions had been present for a mean of 9.9 weeks before treatment started. Those due to Lbb were larger yet they had been present for a shorter time than those due to $\mathrm{Lmm}$, indicating faster growth. There were no other distinguishing features between them.

5. Treatment with sodium stibogluconate was effective. Two regimens were used consisting of either $600-800 \mathrm{mg}$ daily given initially for 10 days, or $600 \mathrm{mg}$ bd given initially for 14 days. Of those allocated to the lower dose regimen $48.5 \%$ were cured after the initial 10 day course and ultimately the ulcers of $93 \%$ healed following more prolonged treatment at that dose. Of those allocated to the higher dose regimen $63.9 \%$ were cured after the initial 14 day course and ultimately the ulcers of all these soldiers healed after more prolonged treatment at that dose. In both regimens $90 \%$ of patients were cured when a total of $24 \mathrm{~g}$ of sodium stibogluconate had been given.

6. Soldiers did not tolerate the higher dose regimen as well as the low dose treatment - reports of myalgia, anorexia and malaise were more frequent in those 
who received the higher dose. A transient leucopenia and a rise in serum aminotransferases was noted during treatment. These changes were dose dependent but appeared to be settling 2 weeks after treatment.

7. No cases of mucocutaneous leishmaniasis were detected. 


\section{CHAPTER 3}

\section{Hepatotoxicity of sodium stibogluconate} therapy.

3.1 Aims of the Investigation

3.2 Introduction

3.3 Methods

3.4 Results

3.5 Discussion

3.6 Summary 


\subsection{Aims of the Investigation}

To determine whether hepatocellular damage occurs during sodium stibogluconate therapy.

To assess the effect of sodium stibogluconate therapy on hepatic metabolic capacity.

\subsection{Introduction}

Sodium stibogluconate is the mainstay of treatment for most forms of leishmaniasis. In recent years higher doses have been administered and courses have lasted longer in order to achieve greater efficacy. A rise in serum aminotransferases, ALT and AST, have been noted during treatment for cutaneous disease. The results of the retrospective study described in chapter 2, and those of Ballou et al (1987), indicate that these changes may be dose-related. Increased serum aminotransferase levels indicate hepatocellular damage but there is little correlation between their absolute level and the degree of damage (Hayes \& Bouchier 1989). It is therefore important, particularly with the higher doses now in vogue, to determine whether significant hepatocellular damage occurs during sodium stibogluconate therapy and whether it is reversible after therapy has been stopped.

Glutathione S-transferase B1 (GST, EC 2.5.1.18) has been shown to be a very sensitive index of hepatocellular damage. Indeed, marked elevations may be observed when other indices of liver damage remain normal (Bass et al 1978, Beckett et al 1985a, Beckett et al 1985b, Beckett \& Kellet et al 1985, Beckett \& Hayes 1993). Furthermore, unlike aminotransferase levels, plasma GST levels correlate with the histological changes observed in patients with chronic liver disease (Sherman et al 1983).

The predominant GST isoenzymes found in human liver are cationic and comprise at least 2 distinct subunits, $B_{1}$ and $B_{2}$ ( Stockman et al 1985). These basic isoenzymes, like all basic cytosolic GST, are dimeric and the $\mathrm{B}_{1}$ 
and $B_{2}$ subunits combine to form dimers. Measurement of either of these subunits is a more sensitive index of hepatocellular damage than serum aminotransferase activity (Beckett et al 1985b, Beckett \& Hayes 1987). This greater sensitivity may be related to their intra-hepatic distribution. The aminotransferases, GGT, and ALK P are predominantly localised to the periportal hepatocytes and provide satisfactory markers of damage to this zone of the liver, they are poor indicators of alcohol and drug-related liver damage that may occur in centrilobular hepatocytes; frank centrilobular necrosis may occur without any abnormalities being evident in the plasma aminotransferases (Rappaport 1980). In contrast, GST $B_{1}$ is found in very high concentrations in hepatic cytosol and it is distributed evenly throughout the entire liver lobule (Redick et al 1982, Hiley et al 1989). A further advantage of GST over aminotransferases is its very short half-life in plasma (less than 1 hour); it is therefore able to detect when hepatocellular damage stops with more precision (Adachi et al 1980, Beckett \& Hayes 1987, Beckett \& Hayes 1993). Assay of plasma GST has been used to detect hepatocellular damage in a variety of diseases and also following toxic damage due to alcohol, halothane and paracetamol (Beckett et al 1985a, Hussey et al 1986, Beckett \& Hayes 1987, Howie et al 1989, Hayes et al 1990).

Although aminotransferases and GST will detect hepatocellular damage, it is more important, clinically, to determine whether or not the metabolic capacity of the liver is impaired. The large metabolic reserve of the liver, particularly in fit young men, means that the commonly measured parameters of hepatic synthetic capacity, such as the prothrombin time and serum albumin, do not become abnormal until the damage is extensive (Preisig 1986). This problem has been solved by the development of clearance tests, analogous to the creatinine and inulin clearance tests used to assess renal function (Preisig 1986, Hayes \& Bouchier 1989, McIntyre \& Rosalki 1991). Hepatic clearance depends upon 2 major factors: (a) hepatic blood flow ( $Q$ ) and, (b) extraction during passage through the liver $(E)$ :

$\begin{aligned} & \text { Hepatic } \\ & \text { clearance }\end{aligned}=\begin{aligned} & \text { Blood } \\ & \text { flow }(\mathrm{Q})\end{aligned} \quad \times \quad \begin{aligned} & \text { Hepatic } \\ & \text { extraction(E) }\end{aligned}$


where

$\begin{aligned} & \text { Hepatic } \\ & \text { extraction } \\ & (E)\end{aligned}=\frac{\begin{array}{c}\text { inflow } \\ \text { concentration }\end{array}-\begin{array}{c}\text { outflow } \\ \text { concentration }\end{array}}{\text { inflow concentration }}$

The tracer used must be excreted only by the liver.

Tracers can be divided into 2 types: (a) flow dependent tracers - those in which clearance varies with hepatic blood flow (i.e. E approaches unity) and, (b) capacity limited - those in which clearance is independent of hepatic blood flow (i.e. $E$ is small). Capacity limited tracers are required to assess metabolic capacity. Caffeine is an ideal tracer: it is readily available, it is well absorbed orally, in the doses required it is completely innocuous, it is solely metabolised in the liver (by cytochrome $\mathrm{P}_{4501 \mathrm{~A} 2}$ ) and it has a low extraction (E) ratio. It has been shown that the clearance rate of caffeine from the blood, following oral administration, directly reflects the amount of functioning liver tissue. Furthermore, there is no overlap in clearance rates between healthy subjects and patients with cirrhosis (Hayes \& Bouchier 1989, Kalow \& Tang 1993, Preisig 1986, Renner et al 1984).

In this study hepatocellular damage and metabolic capacity were prospectively assessed in patients treated for $\mathrm{CL}$ with either sodium stibogluconate or aminosidine.

\subsection{Methods}

Thirteen consecutive British soldiers enrolled into the prospective, randomised trial comparing the efficacy of sodium stibogluconate with aminosidine for the treatment of Belizian CL (fully described in chapter 5) entered this study. Each soldier gave his informed consent. Treatment with either sodium stibogluconate $20 \mathrm{mg} / \mathrm{kg} /$ day (Pentostam ${ }^{R}$, Wellcome,UK) or aminosidine $14 \mathrm{mg} / \mathrm{kg} /$ day (max $1 \mathrm{~g} /$ day) (Gabbromicina, Farmitalia Carlo Erba, Italy). Both drugs were given for 20 days by iv infusion: sodium stibogluconate was given undiluted at $1 \mathrm{ml} /$ minute whereas aminosidine was 
diluted in $400 \mathrm{ml} \mathrm{N}$ saline and given over 3 to 4 hours. Routine clinical and laboratory monitoring was performed as described in chapter 5 . Ulcers were considered to have clinically healed when they had re-epithelialised and the scar was flat and non-indurated.

Routine liver function tests (bilirubin, AST, ALT, GGT, ALK P and albumin) were measured using an automatic analyser (BM/Hitachi 747, Boehringer Mannheim, Mannheim, Germany) and plasma glutathione S-transferase B1 (GST, E.C.2.5.1.18) was measured by radioimmunoassay (Hayes et al 1983). The caffeine clearance test was performed according to the method described by Setchell et al (1987): subjects were fasted overnight, a single dose of $225 \mathrm{mg}$ caffeine was administered in a cup of decaffeinated coffee which was consumed over 5 minutes. Blood samples were taken at $0,60,90,120$ and 180 minutes and the caffeine level measured by high performance liquid chromatography using an Altex 320 (Anachem, Luton, UK). All tests were performed before treatment, at the end of treatment and 6 weeks after treatment had stopped.

Five of these soldiers had been treated with sodium stibogluconate, 6 with aminosidine and 2 soldiers were first treated with aminosidine and then, because of their poor response, with sodium stibogluconate after a 6 week break. The clinical features of these soldiers at the start of treatment and their response to treatment are shown in table 3:1.

Statistical analysis. Changes in ALT, GST and caffeine clearance before and at the end of treatment, and also before and 6 weeks after treatment were analysed by a Wilcoxon matched-pairs test.

\subsection{Results}

The variations in the activity of ALT, the concentration of GST and the caffeine clearances for each individual within each treatment group are shown in figures 3:1, 3:2 and 3:3 respectively; the data can be found in appendix 2:1. Sodium stibogluconate therapy was associated with a significant rise in ALT $(p<0.05)$ and GST $(p<0.05)$ and a non-significant fall in caffeine clearance in 
Table 3:1

Clinical features and response to treatment of soldiers in each treatment group.

\begin{tabular}{|c|c|c|}
\hline & \multicolumn{2}{|c|}{ Treatment Group } \\
\hline & Sodium Stibogluconate & Aminosidine \\
\hline Number of soldiers & $7^{*}$ & 8 \\
\hline Mean age (years) & 23.6 (sd 3.3) & $25.0(\mathrm{sd} 2.7)$ \\
\hline Mean weight $(\mathrm{kg})$ & $72.0 \quad(\mathrm{sd} 5.9)$ & $72.2(\mathrm{sd} 6.5)$ \\
\hline Mean number of lesions & $1.3 \quad(\mathrm{sd} 0.8)$ & $1.3 \quad(\mathrm{sd} 0.7)$ \\
\hline Mean duration (weeks) & $16.6(\mathrm{sd} \mathrm{8.5)}$ & $10.6(\mathrm{sd} 4.6)$ \\
\hline Mean size ${ }^{\#}(\mathrm{~mm})$ & $16.0(\mathrm{sd} 2.7)$ & $10.6(\mathrm{sd} 4.6)$ \\
\hline \multicolumn{3}{|l|}{ Site of lesions } \\
\hline Limbs & 5 & 6 \\
\hline Head \& neck & 3 & 3 \\
\hline Trunk & 0 & 1 \\
\hline L-D bodies observed & 7 & 8 \\
\hline Positive culture & 3 & 8 \\
\hline Clinically healed & $6 / 7$ & $5 / 8$ \\
\hline
\end{tabular}

*Two of these soldiers had been treated with aminosidine first. \#longest axis 
Figure 3.1: Changes in ALT before treatment, at the end of treatment, and 6 weeks after treatment stopped for soldiers treated with sodium stibogluconate (top) or aminosidine (bottom).
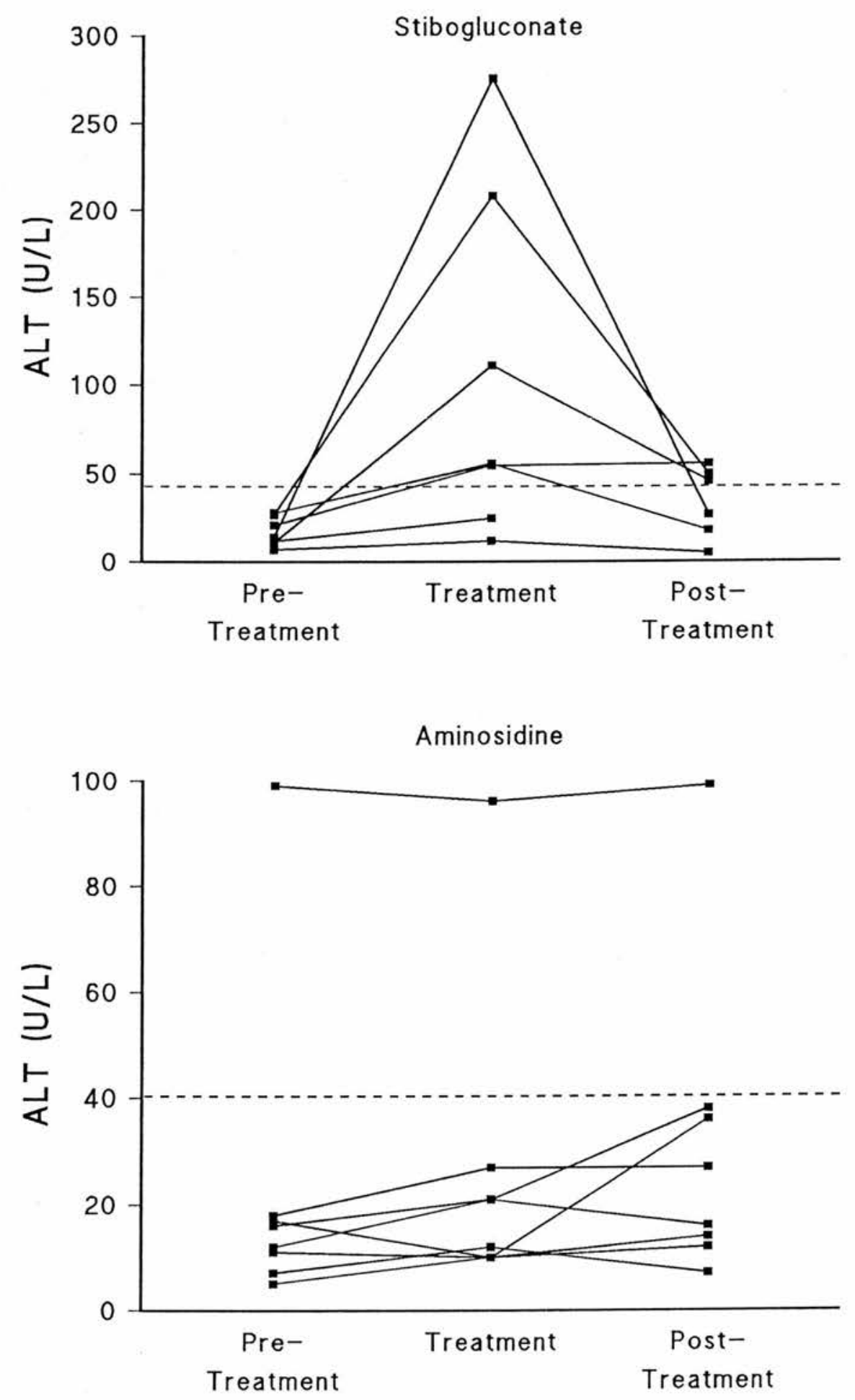
Figure 3.2: Changes in GST before treatment, at the end of treatment, and 6 weeks after treatment stopped for soldiers treated with sodium stibogluconate (top) or aminosidine (bottom).

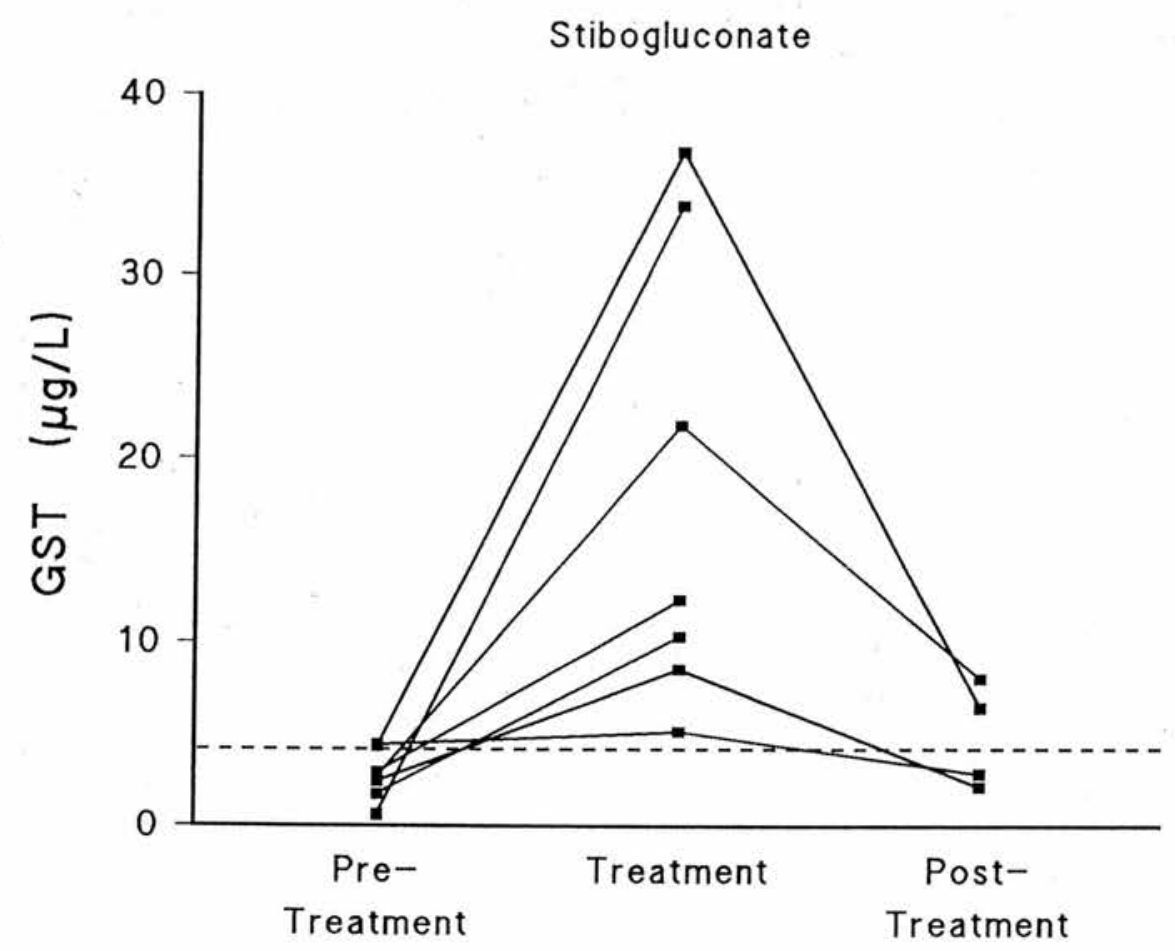

Aminosidine

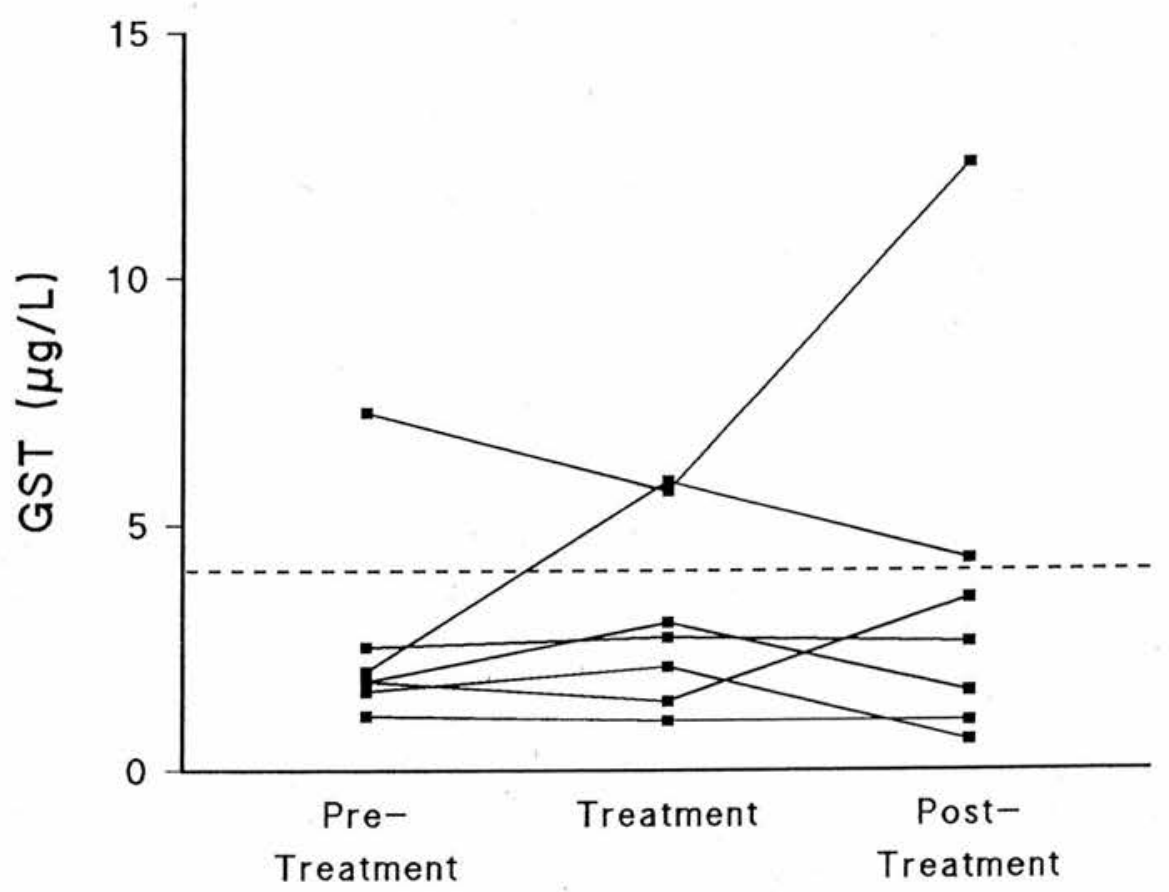


Figure 3.3: Changes in caffeine clearance before treatment, at the end of treatment, and 6 weeks after treatment stopped for soldiers treated with sodium stibogluconate (top) or aminosidine (bottom).
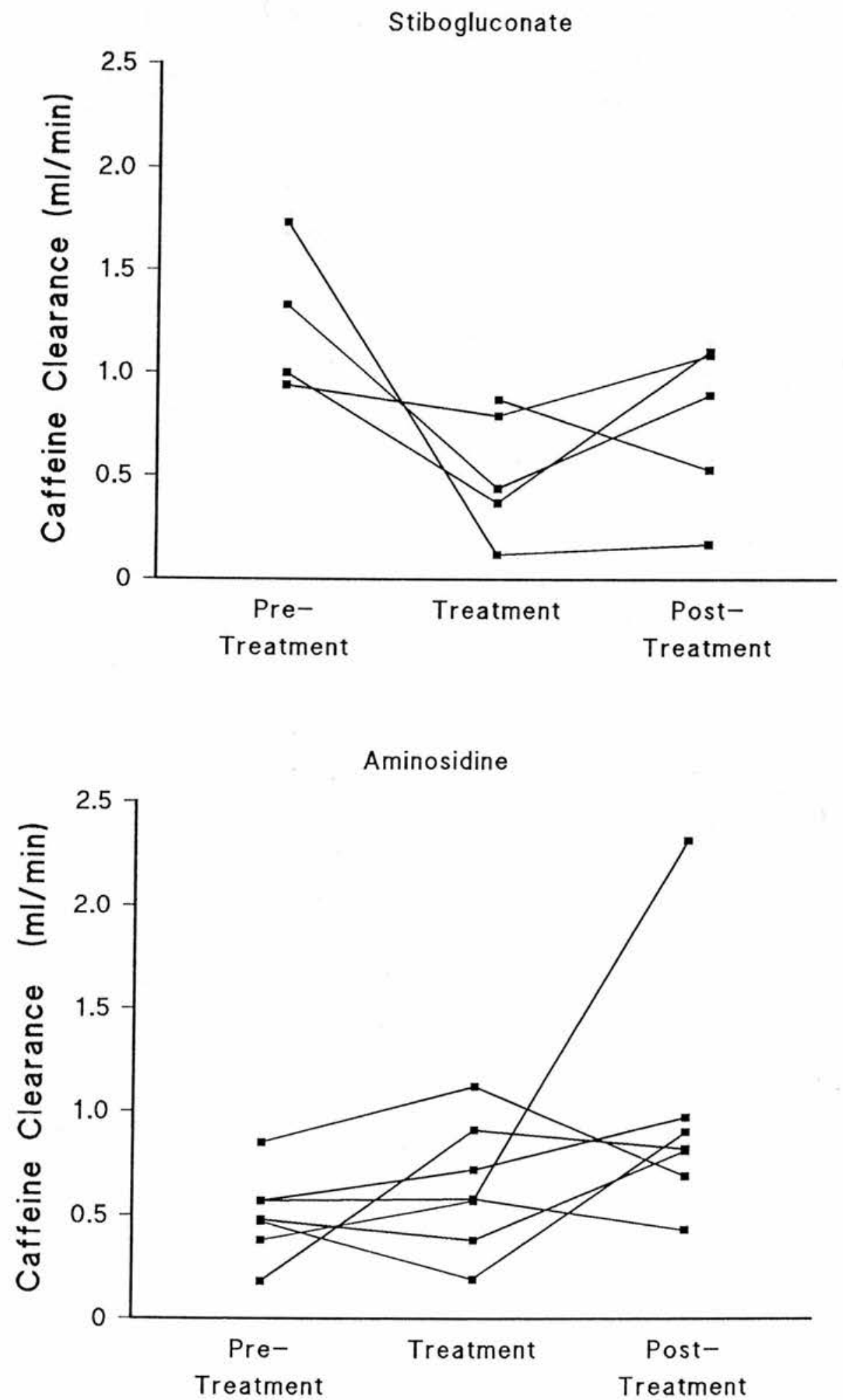
every case. Log ALT correlated with log GST ( $r=0.87, n=40)$. The prothrombin time and serum albumin did not change. Six weeks after treatment stopped the ALT and GST had returned to near pre-treatment levels in every patient as had the caffeine clearance in all cases except one. This soldier had the greatest fall in caffeine clearance and his GST had increased from $1.7 \mathrm{ug} / \mathrm{l}$ to $10.3 \mathrm{ug} / \mathrm{l}$.

Aminosidine was not associated with a rise in ALT or a fall in caffeine clearance. In one soldier a small rise in GST was noted and in a further soldier, who was reported to consume large quantities of alcohol, the ALT and GST were elevated before treatment, fell during treatment (alcohol was forbidden during treatment which was supervised in hospital) but rose again after discharge. Acute alcohol ingestion in heavy drinkers leads to increased GST levels and their basal levels are often raised (Beckett \& Hayes 1993).

\subsection{Discussion}

An increase in serum aminotransferases, indicative of hepatocellular damage, has been reported during treatment of $\mathrm{CL}$ with sodium stibogluconate in American soldiers (Ballou et al 1987) and it was also found in the retrospective study of British soldiers in whom the elevation appeared to be dose-dependent (chapter 2). The clinical significance of this observation is not known. It is not possible to separate toxic from therapeutic effects on the liver in visceral leishmaniasis but this should not be a problem in cutaneous disease. Nevertheless, a further control group of soldiers with $\mathrm{CL}$ who were treated with aminosidine, which is not known to be hepatotoxic, was incorporated into this study. GST is a very sensitive and specific indicator of hepatocellular damage and its short half-life in plasma allows early recognition of when active cellular damage has ceased (Beckett \& Hayes 1993). Treatment with sodium stibogluconate, but not aminosidine, resulted in significant increases in ALT and GST confirming that sodium stibogluconate causes hepatocellular damage. The elevation of both ALT and GST indicate that damage was occurring either in all zones of the liver or that it was confined to the periportal hepatocytes. The fall in both ALT and GST to 
pre-treatment levels 6 weeks after treatment stopped indicates that damage had stopped.

The fall in caffeine clearance with sodium stibogluconate indicates that the metabolic capacity of the liver was reduced. The numbers in this study were insufficient to reach statistical significance but caffeine clearance fell in every case. In most cases this appears reversible. Although the fall in clearance could be accounted for by selective inhibition of cytochrome P-450 1A2, the principle pathway by which caffeine is metabolised (Kallow \& Tang 1993), the concomitant changes in ALT and GST indicate that hepatocellular injury is the probable mechanism. These change were not observed in soldiers treated with aminosidine. Sodium stibogluconate is, therefore, implicated as the cause.

Hepatotoxicity must therefore be added to the list of complications associated with sodium stibogluconate therapy. In most cases of $C L$ this will be of limited clinical significance, but for those with pre-existing liver disease, and possibly those with visceral disease, alternative therapy should be considered. In all cases the concurrent use of other potentially hepatotoxic agents should be avoided. The ALT gives a practical indication of the severity of acute hepatocellular damage, but it will not detect any cases who sustain permanent damage. The nature and extent of this hepatotoxicity require further studies. The number of patients studied was small and not all patients were studied before treatment, at the end of treatment and 6 weeks after treatment. A larger study along similar lines would confirm and quantify the acute toxicity. In addition, however, the caffeine clearance test should also be repeated 6 months after treatment to determine whether there is a persistent impairment of metabolic capacity. 
3.6 Summary: Hepatotoxicity of sodium stibogluconate therapy.

1. Sodium stibogluconate causes acute hepatocellular damage in a dose of $20 \mathrm{mg} / \mathrm{kg} /$ day. The damage stops when treatment stops.

2. The ALT appears to be a reasonable measure of the amount of acute damage.

3. The available data indicate that there is a fall in the metabolic capacity of the liver during treatment with sodium stibogluconate.

4. Aminosidine is not hepatotoxic. 


\section{Chapter 4}

\section{Cardiotoxicity of sodium stibogluconate.}

4.1 Aim of the investigation

4.2 Introduction

4.3 Methods

4.4 Results

4.5 Discussion

4.6 Summary 


\subsection{Aim of the investigation}

To detect and assess the cardiac effects of sodium stibogluconate therapy in a dose of $20 \mathrm{mg} / \mathrm{kg} /$ day for 20 days.

\subsection{Introduction}

Antimony is cardiotoxic. In dogs it reduces myocardial contractile force (Bromberger-Barnea \& Stephens 1965) and in man the trivalent antimonials have been associated with hypotension, bradycardia, ventricular tachycardia, syncope, electrocardiographic (ECG) changes (deformed P-waves, QT prolongation and T-wave flattening) and occasionally sudden death (Honey 1960, Davis 1961, Somers \& Rosanelli 1962, Surawicz 1970, Sapire \& Silverman 1970, Stemmer 1976). Sodium stibogluconate was derived from the trivalent antimonials as a safer alternative (Anonymous 1961, Stemmer 1976) but T-wave flattening and QTc prolongation during therapy have been reported (Dempsey 1965, Kaplan et al 1978) and appear dose related (Chulay et al 1985); similar changes have also been observed during meglumine antimoniate therapy (Antezana et al 1992). It is not known if these changes occur in association with dysrhythmias, other markers of myocardial electrical instability, or changes in cardiac function although a study of 22 British soldiers treated with a 10 day course of sodium stibogluconate (10 $\mathrm{mg} / \mathrm{kg} / \mathrm{day}$ ) noted transient ECG changes but no change in either heart rhythm or echocardiographic parameters (Henderson \& Jolliffe 1985).

Current regimes recommend the administration of prolonged high dose sodium stibogluconate in order to increase efficacy (WHO 1990, Ballou et al 1987, Hepburn et al 1993). As the ECG changes are dose related (Chulay et al 1985) it was important to determine whether these intensive regimes are associated with significant deleterious effects on cardiac function.

I therefore performed a comprehensive, non-invasive, cardiac assessment in 12 soldiers before, during and after treatment with sodium stibogluconate (20 $\mathrm{mg} / \mathrm{kg} /$ day for 20 days). In addition to routine haemodynamic, biochemical 
and electrocardiographic recordings, including 24 hour ambulatory recordings, detailed assessments of left ventricular function, in both systole and diastole, were made by systolic time interval analysis. The autonomic nervous system is deranged in chronic heart failure (Nolan et al 1992), therefore a measure of cardiac autonomic control was obtained by analysing the heart rate variability over a 24 hour period (Ewing et al 1984, Ewing 1992).

Systolic Time Interval analysis. This technique relates echocardiographic measurements of chamber size and blood flow to the cardiac cycle giving sensitive indices of ventricular performance, thereby permitting detection of any changes in cardiac function occurring as a result of therapy (Lewis et al 1974, Nishimura et al 1989).

A M-mode echocardiograph (Figure 4.1) gives details of chamber size at each point in the cardiac cycle together with the duration of each phase. In addition to these measurements, the fractional shortening and ejection fraction can be calculated using the formulae:

$\begin{aligned} & \text { Fractional } \\ & \text { Shortening }\end{aligned}=\quad \frac{\text { LVDD - LVSD }}{\text { LVDD }}$

where: $\quad L V S D=$ left ventricular systolic dimension

LVDD = left ventricular diastolic dimension

Ejection

Fraction

where:

$$
=\quad \text { LVDV - LVSV }
$$

LVSV

(these volumes are calculated by cubing the appropriate dimension) 
Figure 4.1a: Aortic M- mode echo showing the parasternal short axis view at the level of the papillary muscles showing the cavity size measurements.

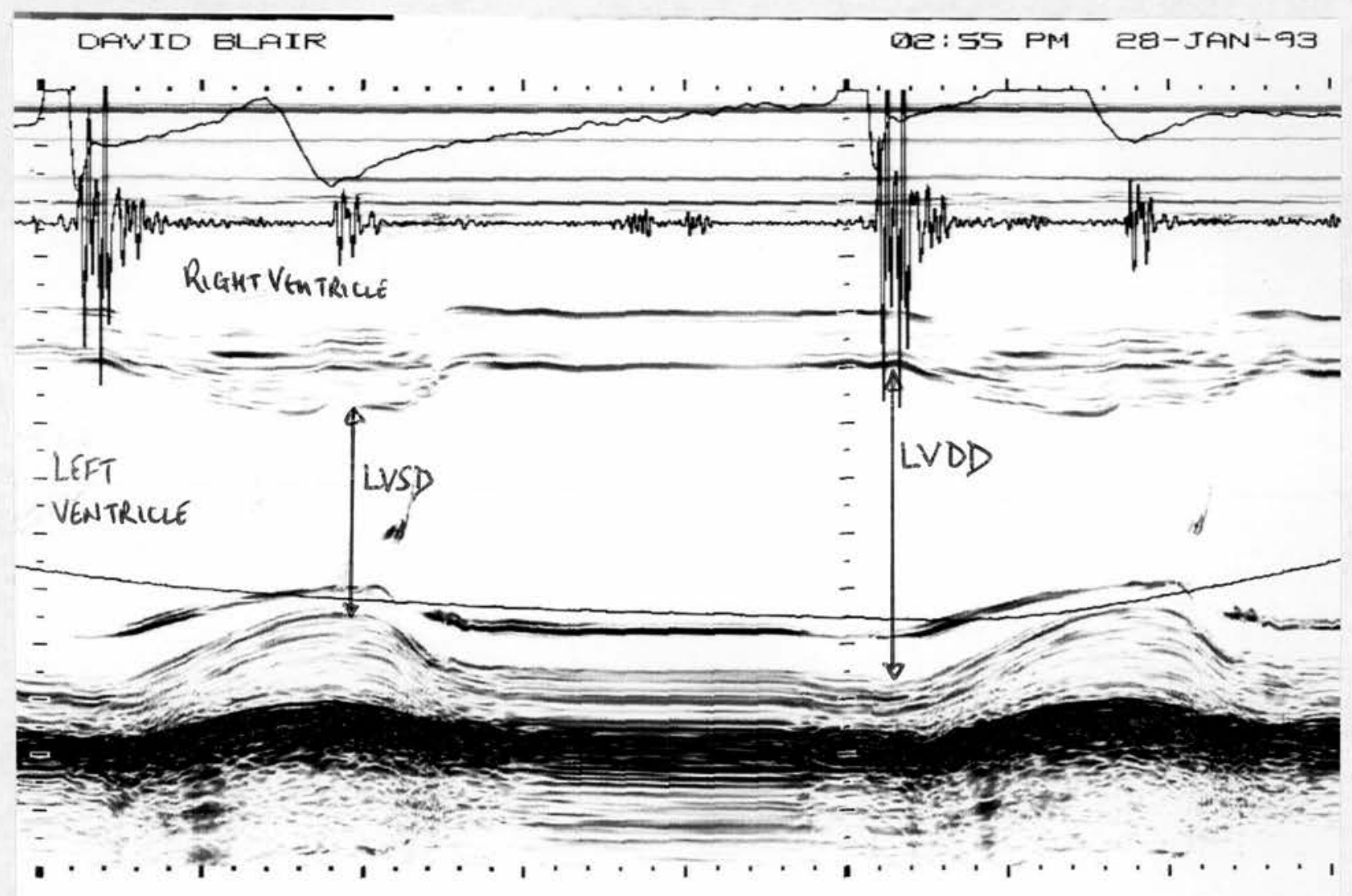


Figure 4.1b: Aortic M-mode echo showing the parasternal view at the level of the aortic root (top) and a styalised recording showing the systolic time intervals (bottom).

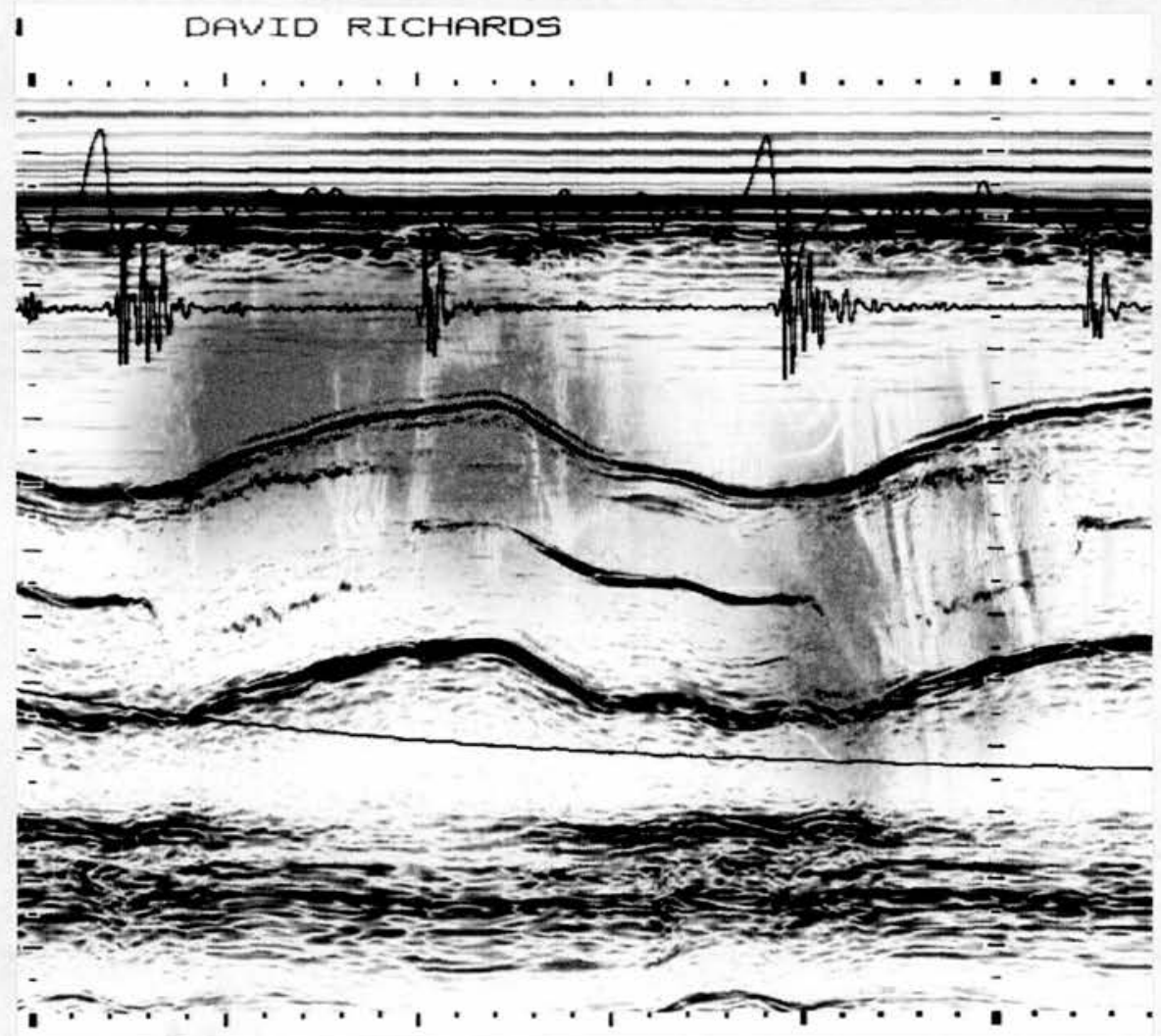

AORTIC M-mode echo: Systolic Time Intervals

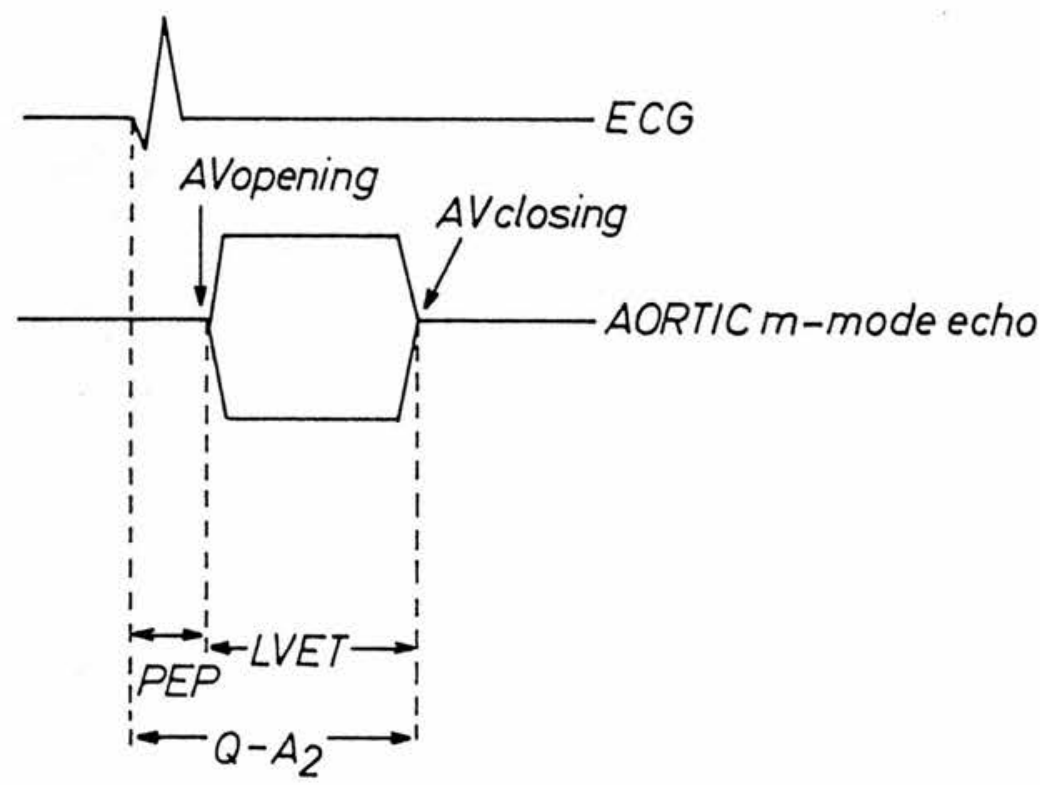


Figure 4.2: Mitral Doppler recording (top) with a styalised representation (bottom).

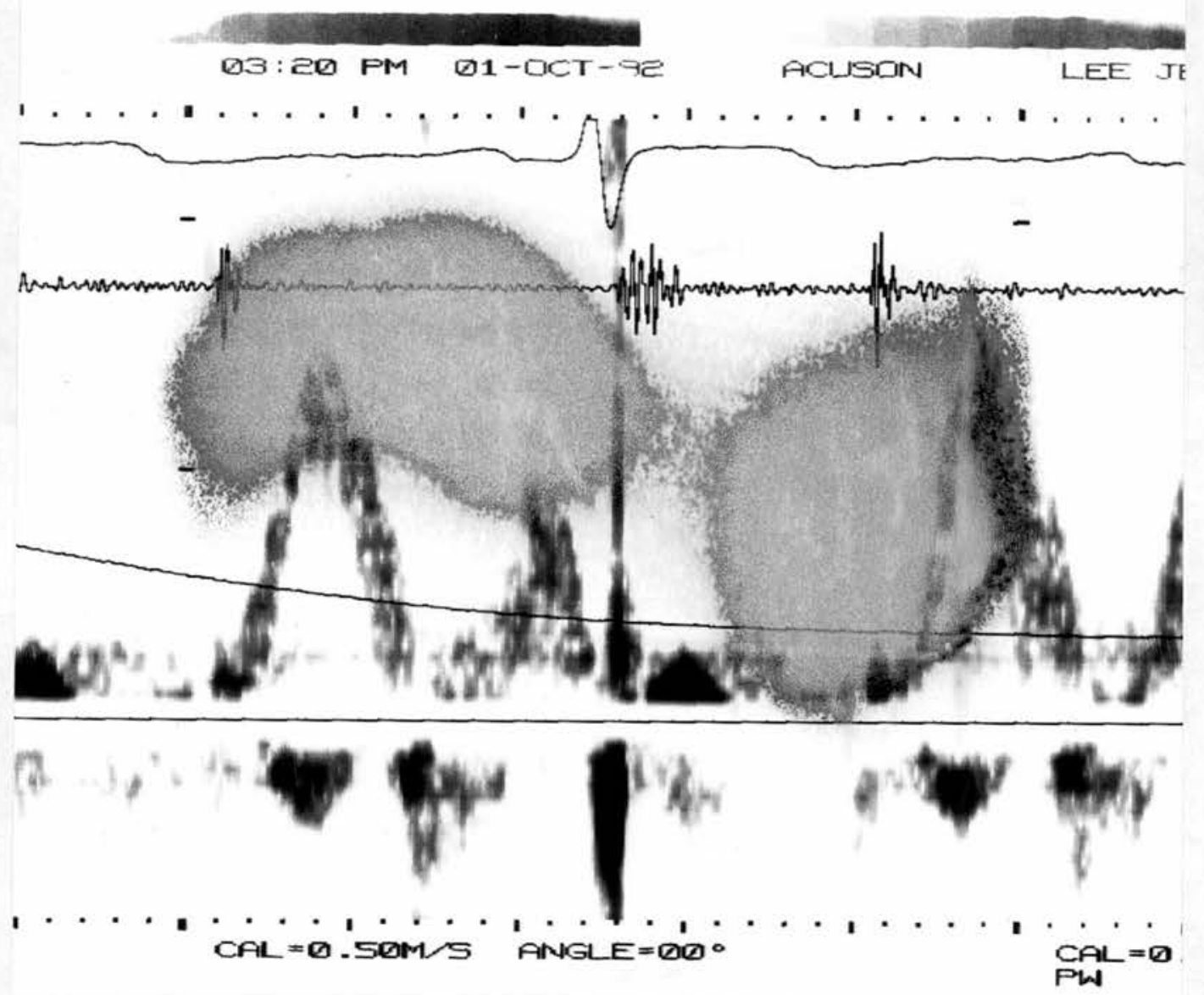

Styalised Doppler Trace With Combined Phono

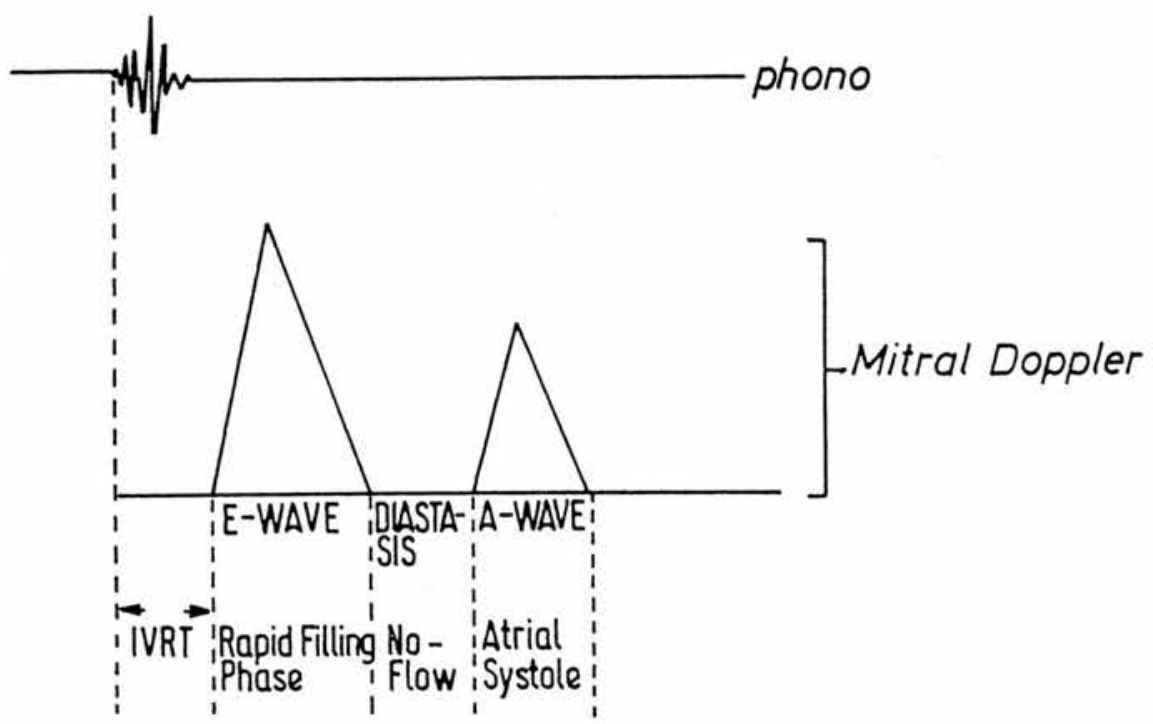


Drug-induced impairment of left ventricular systolic function would be indicated by a:

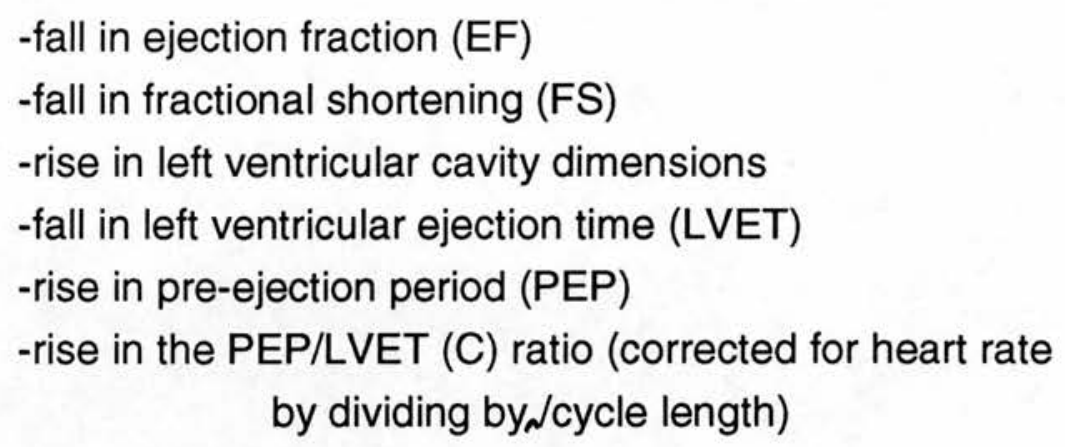

A Doppler echocardiograph (figure 4.2) gives information about flow rates at each phase of the cardiac cycle and this is particularly useful in assessing diastolic function. There are 4 phases to diastole:

Isovolumic relaxation time (IVRT)

-this is the period when the left ventricle is relaxing but all the valves are closed (therefore pressure is falling but the volume is constant).

Rapid early filling phase (E-wave)

-most of left ventricular filling ( $70-80 \%)$ occurs early in diastole when a large pressure gradient exists from left atrium to left ventricle.

Diastasis period

-the point at which left atrial pressure falls to left ventricular pressure and flow stops.

Late atrial filling (A-wave)

-the atrium contracts and the final $20-30 \%$ of left ventricular filling occurs prior to systole.

Abnormalities of left ventricular diastolic function often precede abnormalities of systolic function (Bonow et al 1981a, Bonow et al 1981b, Clarkson et al 1994) and may therefore be more sensitive to small drug induced changes in myocardial performance than measures of global systolic function. The pressures are relatively low, therefore it is necessary to measure each parameter during inspiration, expiration and apnoea. Drug-induced 
impairment of diastolic function would be indicated by an increase in stiffness of the ventricle with consequent :

-increase in the IVRT

-fall in the rate of ventricular filling

\subsection{Methods.}

Subjects and drug regime. Twelve British soldiers, aged between 20 and 30 years, who had contracted CL during jungle training in Belize were studied. All the subjects gave informed consent and their characteristics are shown in table 4.1. None had pre-existing cardiac disease, renal disease, diabetes mellitus or alcohol abuse. Those receiving concurrent medication were excluded from this study. All subjects had a history of exposure to the parasite, a typical skin lesion and in every case it was confirmed by identification of LD bodies and in 9 cases also by culture. Treatment with sodium stibogluconate (Pentostam R, Wellcome, UK. batch no. A1058A, manufactured January 1991 and stored at below $25^{\circ} \mathrm{C}$ ) $20 \mathrm{mg} / \mathrm{kg} /$ day was administered by i.v. infusion at $1 \mathrm{ml} / \mathrm{min}$ for 20 days.

\section{Blood pressure and biochemical measurements were made before} treatment started (day 0 ), weekly during treatment (days 7,14 ), on the last day of treatment (day 20), 2 weeks after treatment stopped (day 34) and 6 weeks after treatment stopped (day 62). Blood pressure was measured at rest using a mercury sphygmomanometer (diastolic phase V). Creatine kinase and urea-stable lactate dehydrogenase were measured using commercial photometric assays (Monarch, Allied Instrumentation Laboratory, Warrington UK).

Electrophysiological measurements. Standard resting ECGs were recorded using a Minigraph 12 lead recorder (Cardiac Recorders, London, UK) before treatment weekly during treatment (days 7, 14, \& 20) and at 2 and 6 weeks after treatment stopped. The ECGs were inspected and the cardiac axis determined using standard criteria (Fisch 1992). The ECGs were then projected onto a screen to produce a magnification of $x 5$. Using the isoelectric PR and post-T wave segments as the baseline, the height of the Rwave in $V_{1}$ and the sum of the heights of the $T$-waves in $V_{4}-V_{6}$, together with 
their calibration recordings, were measured directly using a ruler and used as quantitative measurements of changes in QRS and T wave magnitude.

Twenty-four hour ambulatory ECGs were obtained, during normal ambulatory activity, from each subject before treatment, on the last day of treatment (day 20) and 6 weeks after treatment stopped (day 62) using a miniature tape recorder (Tracker, Reynolds Medical Ltd, Hereford. U.K.). Times of going to bed and getting up were noted by the subjects. The tapes were analysed using a Pathfinder arrhythmia analyser (Reynolds Medical Ltd, Hereford. U.K.) at 120 times the original recording speed.

Supraventricular and ventricular ectopic beats were detected due to differences in their timing and morphology, compared with continuously updated characteristics of normal sinus beats (Neilson 1975). Each analysis was monitored throughout by a specialist technician to confirm the ectopic beats and scrutinise $P$ wave morphology to exclude any episodes of nonsinus rhythm. Where less than 24 hours of recording was suitable for analysis the totals were adjusted to the equivalent of 24 hours. Recordings shorter than 18 hours or with less than $40 \%$ of the tape suitable for analysis were rejected. The mean waking and sleeping heart rates over the 24 hour period were calculated, and the number of ectopic beats per 24 hours was counted.

Changes in autonomic activity occur in association with changes in left ventricular function (Nolan et al 1992) and may be important in the pathogenesis of ventricular tachyarrhythmias (Verrier \& Lown 1975). During arrhythmia analysis, each normal RR interval was measured, the mean RR interval calculated, and the 24 hour standard deviation (SD) of RR intervals was measured as a general index of sympatho-vagal activity (Murray et al 1975, Ewing 1992). A specific measure of parasympathetic activity was obtained by the $\mathrm{NN}_{50}$ time domain index in which each time the difference between the RR intervals of consecutive normal beats increased by more than 50 milliseconds a count was registered. The results were presented as a total 24 hour count (NN50 count) (Ewing et al 1984, McAreavey et al 1989). 
Assessment of left ventricular function by echocardiography was performed on all subjects before treatment, on the last day of treatment (day 20 ) and 6 weeks after treatment stopped (day 62). All echocardiographic measurements were obtained by the same operator (Mrs L. Fenn), using identical equipment on each occasion, in a temperature-controlled laboratory at $22^{\circ} \mathrm{C}$. Following an overnight fast, the patients rested in the standard left lateral position until their heart rate stabilised. Heart rate was recorded from a continuous ECG (lead 2) and respiration with a respiratory probe. High quality simultaneous recordings of the echocardiogram, ECG, phonocardiogram and respiration tracing were obtained using an Accuson 128/XP10 scanner (Allison, Mount Vernon, USA). Phonocardiograms were obtained with a high impedance piezo-electric microphone with a low frequency filter positioned at the base of the heart. Aortic valve closure was taken as the start of the first high frequency vibration of the aortic component of the second heart sound, checked for validity with the aortic echocardiogram and aortic artefact on the Doppler recording. Two dimensional, M-mode and Doppler echocardiograms were obtained with a hand-held 3.5 $\mathrm{MHz}$ phased array transducer during quiet respiration. The simultaneous recordings were printed onto thermal paper at $100 \mathrm{~mm} / \mathrm{s}$. Only subjects in whom clear continuous echos from the septum and posterior wall, and in whom easily recognisable aortic valve opening and closure were visible were used for the study. Pulsed Doppler recordings of the mitral inflow velocity profile were obtained by positioning the sample volume at the level of the mitral leaflet tips in diastole in the apical four chamber view. Care was taken to obtain a smooth velocity curve with well defined peaks and minimal spectral broadening. Recordings were analysed using a sonic pen and a digitising tablet (Sunnagraphics $0.1 \mathrm{~mm}$ resolution, grid) interfaced to a Microcomputer (Sun $3 / 180$ workstation, Sunnagraphics, Connecticut, USA) using the measurement techniques described below. A minimum of 5 cardiac cycles were digitised for each subject on each occasion.

Left ventricular systolic function. Left ventricular internal cavity dimensions were measured at end-systole and end-diastole using standard criteria from the parasternal short axis view (Sahn et al 1978). The left ventricular ejection fraction was derived from the measurements of left ventricular cavity size using the cube formula and fractional shortening (\%FS) by calculating the 
difference between left ventricular diastolic and systolic dimensions divided by the diastolic dimension (Mason \& Fortuin 1978, Freigenbaum 1986). The left ventricular ejection time (LVET) and the total duration of electromechanical systole were measured from the combined ECGs and aortic echocardiograms (Hirschfeld et al 1975). The ratio of the pre-ejection period (PEP) to the left ventricular ejection time (LVET), corrected for heart rate (PEP/LVET(C)), was calculated as an additional heart rate-independent index of left ventricular systolic function (Benchimol \& Matsuo 1971, Gutgesell et al 1977).

Left ventricular diastolic function. Events during early diastole were characterised by measurement of the isovolumic relaxation time (IVRT) and quantitative analysis of the early (E) Doppler velocity profile. Events during mid to late diastole were quantified by measurement of the diastasis period (the time interval from the termination of early inflow to the onset of late inflow) and quantitative analysis of the late (A) Doppler velocity profile. The $E$ and $A$ waves were quantitatively analysed by measurement of their peak velocities, acceleration and deceleration gradients, acceleration and deceleration times, the area under each peak, and the ratio of peak early to peak late inflow velocities (Nishimura et al 1989, Labovitz \& Pearson 1987, Spirito \& Maron 1988). Measurements were made during inspiration, expiration and inspiratory apnoea.

Statistical analysis was carried out using the statistical package SpSS-PC (SpSS, Chicago, USA). Analysis of variance was used to test for changes of the variables over time, a logarithmic transformation was used during testing of the 'counts'. Linear regression analysis was used to test the fall in the height of the T-waves on the serial ECG recordings. 
Table 4.1

Characteristics of patients.

\begin{tabular}{|c|c|}
\hline Number of Patients & 12 \\
\hline Mean age (years) & 23.7 (sd 3.1) \\
\hline Mean Weight (kg) & 71.7 (sd 3.1) \\
\hline Mean number of lesions & $1.25(\mathrm{sd} \mathrm{0.6)}$ \\
\hline $\begin{array}{l}\text { Mean duration of lesions } \\
\text { (weeks) }\end{array}$ & 21.3 (sd 9.9) \\
\hline Mean size ${ }^{\star}(\mathrm{mm})$ & $16.7(\mathrm{sd} 5.0)$ \\
\hline \multicolumn{2}{|l|}{ Site of lesions: } \\
\hline limbs & 11 \\
\hline head \& neck & 3 \\
\hline trunk & 1 \\
\hline LD bodies seen & $12 / 12$ \\
\hline Positive culture & 9/12 (6 Lbb, 3 Lmm) \\
\hline $\begin{array}{c}\text { Clinical healing of lesion } \\
\text { after treatment }\end{array}$ & $11 / 12$ \\
\hline
\end{tabular}

*longest axis 


\subsection{Results}

The 20 day course of sodium stibogluconate $(20 \mathrm{mg} / \mathrm{kg} / \mathrm{day})$ healed the ulcers of 11 of the 12 subjects. All the subjects reported muscle aches and loss of appetite during treatment but there were no other symptoms or signs, in particular no subject developed any cardiac symptoms.

Blood pressure and heart rate. There was a fall in the systolic and diastolic blood pressure $(P<0.05)$ associated with an increase in heart-rate $(p<0.05)$ at the end of the course of treatment. Blood pressure and heart-rate returned to pre-treatment levels 6 weeks after treatment stopped (tables $4.2 \& 4.3$ ).

Cardiac enzymes. There was no change in either serum levels of creatine kinase or lactate dehydrogenase during treatment. A significant $(P<0.05)$ rise in lactate dehydrogenase was noted at both 2 and 6 weeks after the end of treatment and the soldiers had returned to full physical duties. There was no change in creatine kinase following treatment in 10 soldiers but in two it rose to 1497 and $416 \mathrm{IU} / \mathrm{L}$ respectively 6 weeks after treatment stopped (table 4.2). The soldier who had the highest level also had an elevated waking and sleeping heart rate ( $95 \& 90$ beats $/ \mathrm{min}$ ) at that time compared to his baseline ( $70 \& 51)$ and post-treatment ( $83 \& 62)$ rates. His $\mathrm{CK}$ remained high at 545 IU/L 14 months after treatment but at that time only $2 \%$ was CK-MB indicating most was derived from skeletal muscle.

Electrocardiography. The cardiac electrical axis and R-wave magnitude did not change during this study. There was, however, a steady and significant $(P<0.001)$ decrease in the sum of the heights of the T-waves in V4-6 as treatment progressed followed by a return to pre-treatment levels 6 weeks later (table 4.3, figure 4.2).

A complete series of analysable 24 hour recordings was only available for 7 subjects. In 2 cases a pre- treatment recording was not performed, in the remaining 3 cases one of the series contained less than 18 hours of analysable recording. There was no significant increase in supraventricular or ventricular arrhythmias with treatment. Similarly, neither the 24 hour standard 


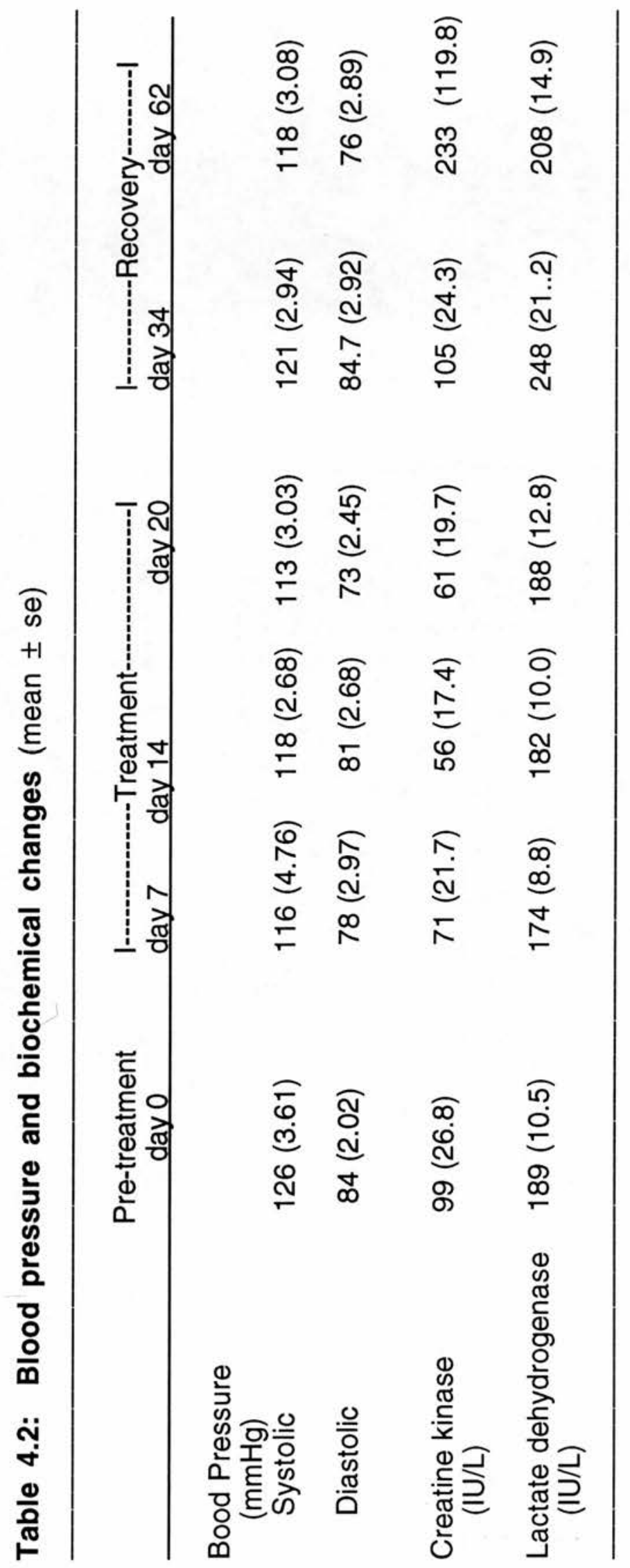




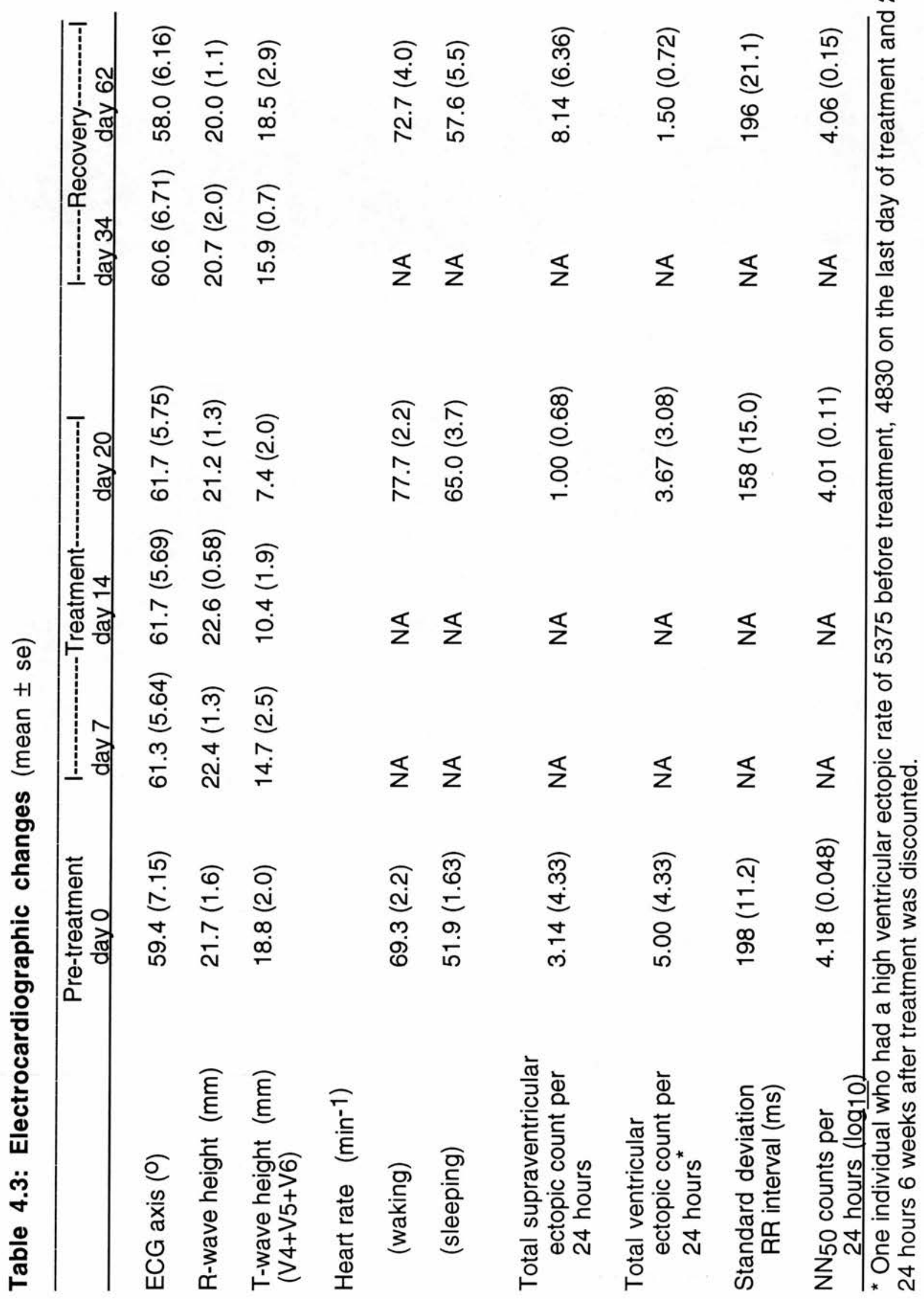




$$
\begin{aligned}
& \text { MIMIIIMn }
\end{aligned}
$$

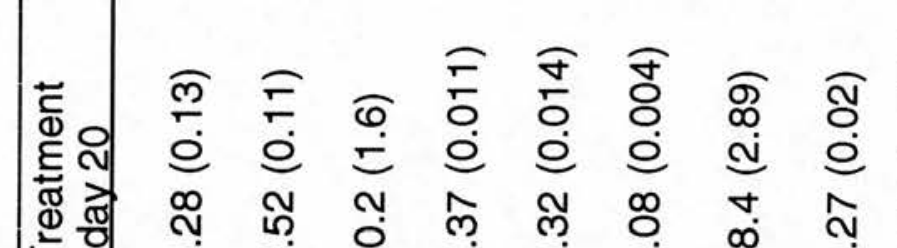

$$
\begin{aligned}
& \text { 可 }
\end{aligned}
$$

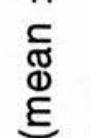

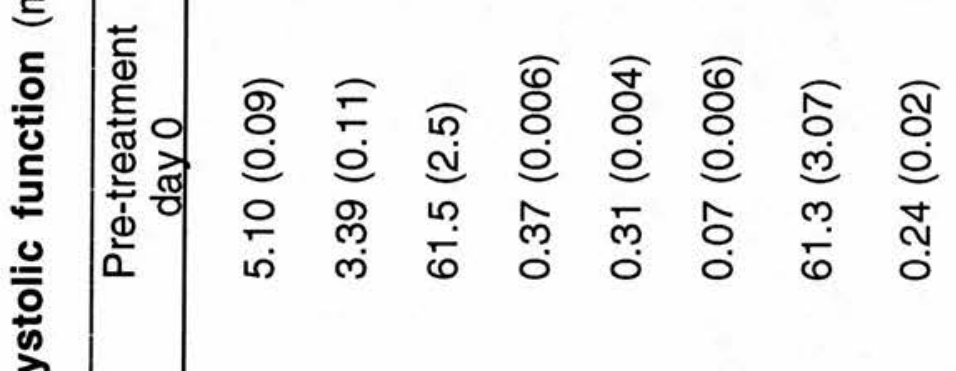

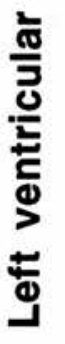

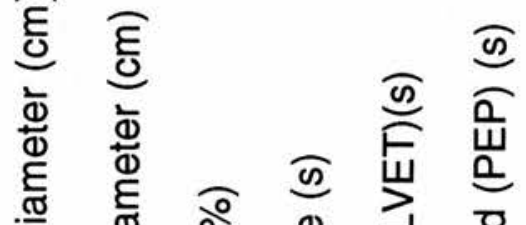

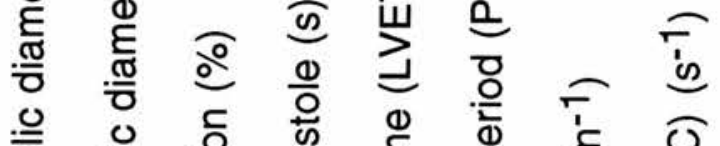

$$
\begin{aligned}
& \text { 高 } \\
& \text { 元 }
\end{aligned}
$$

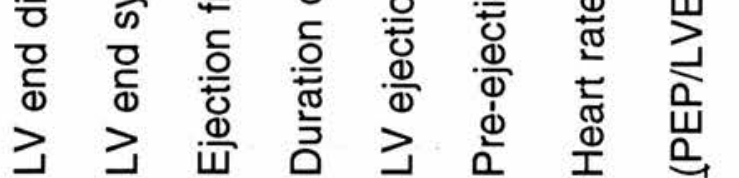




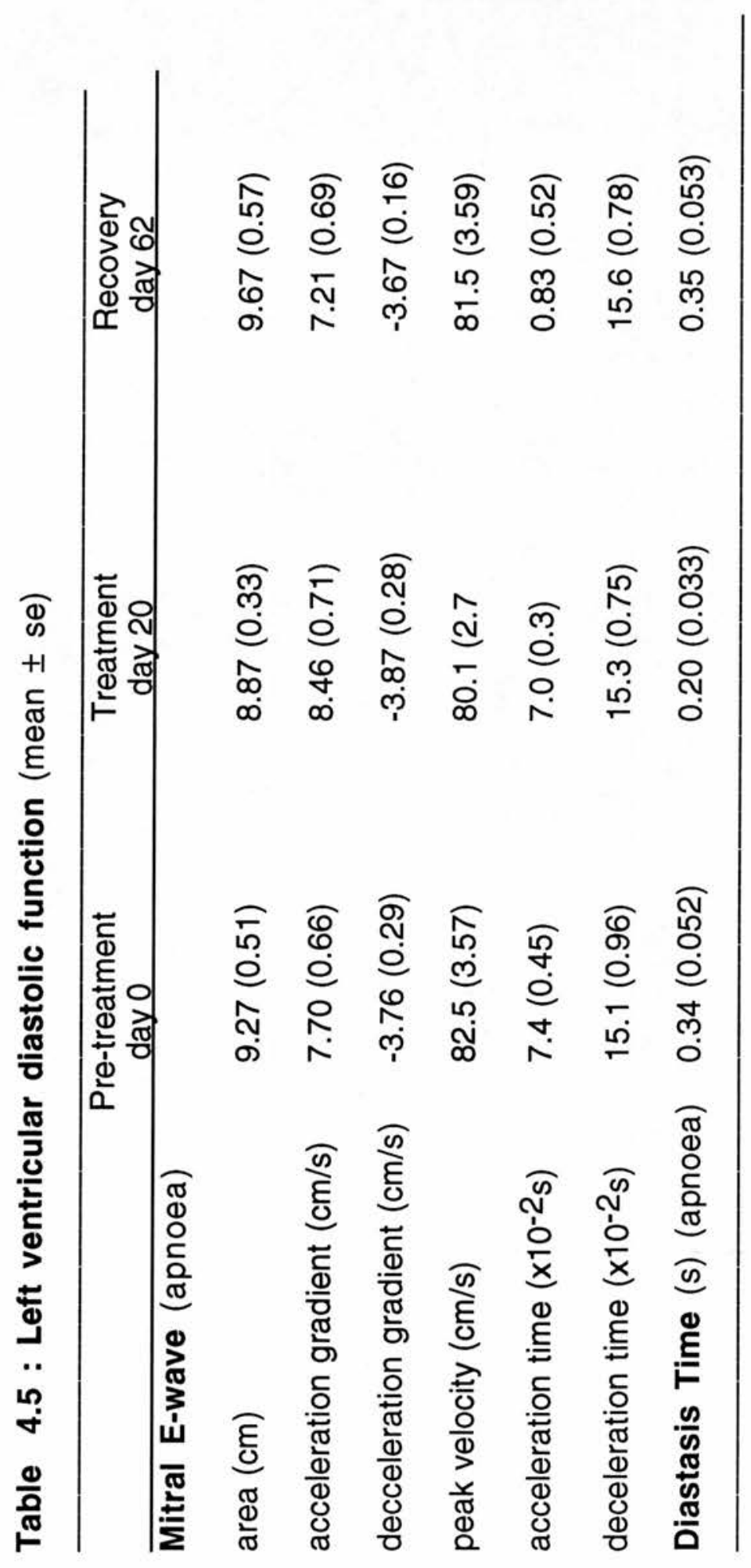




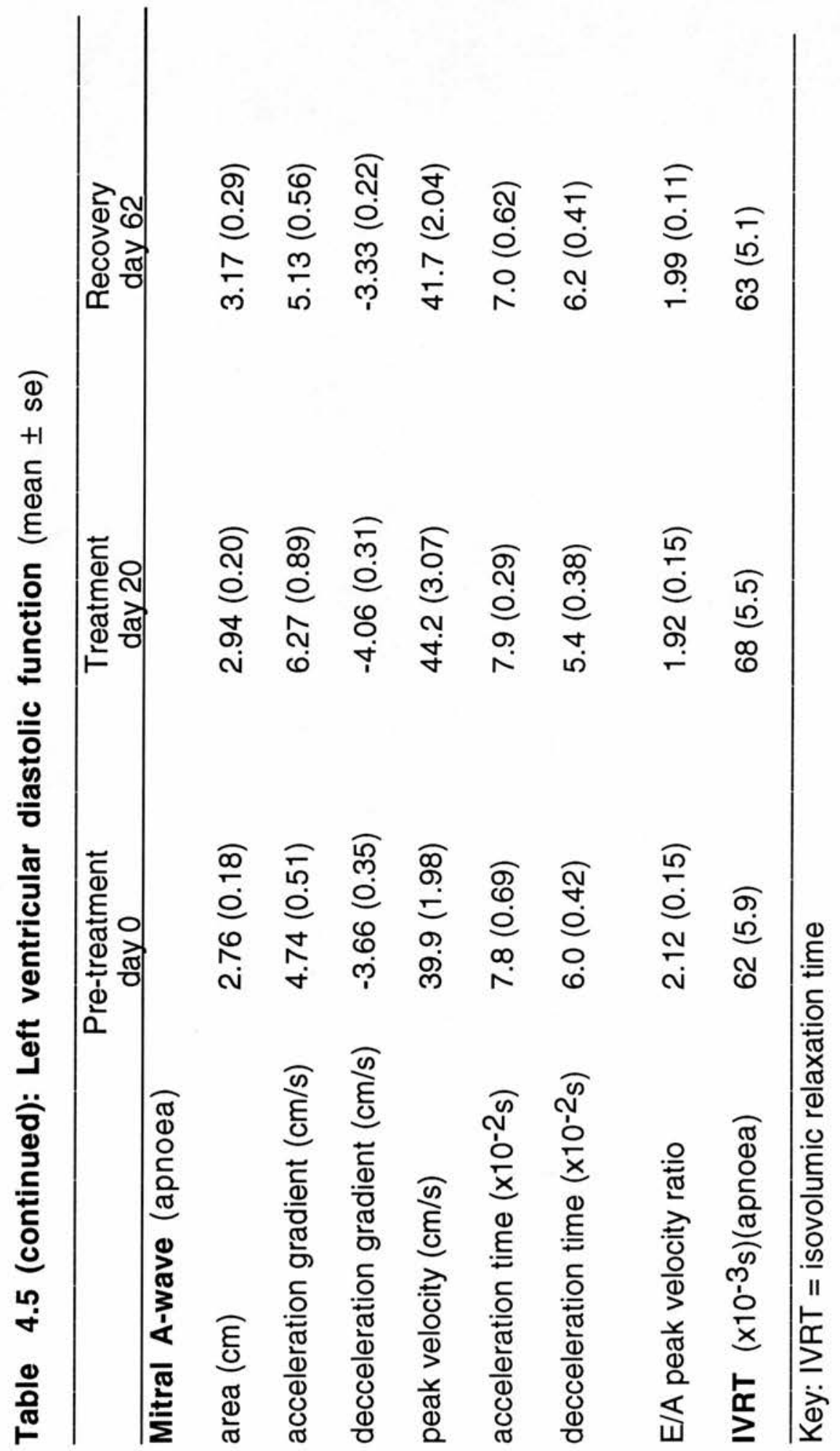


Figure 4.2: The change in the sum of the heights of the $T$ waves in leads V4 + V5 + V6 during treatment with sodium stibogluconate.

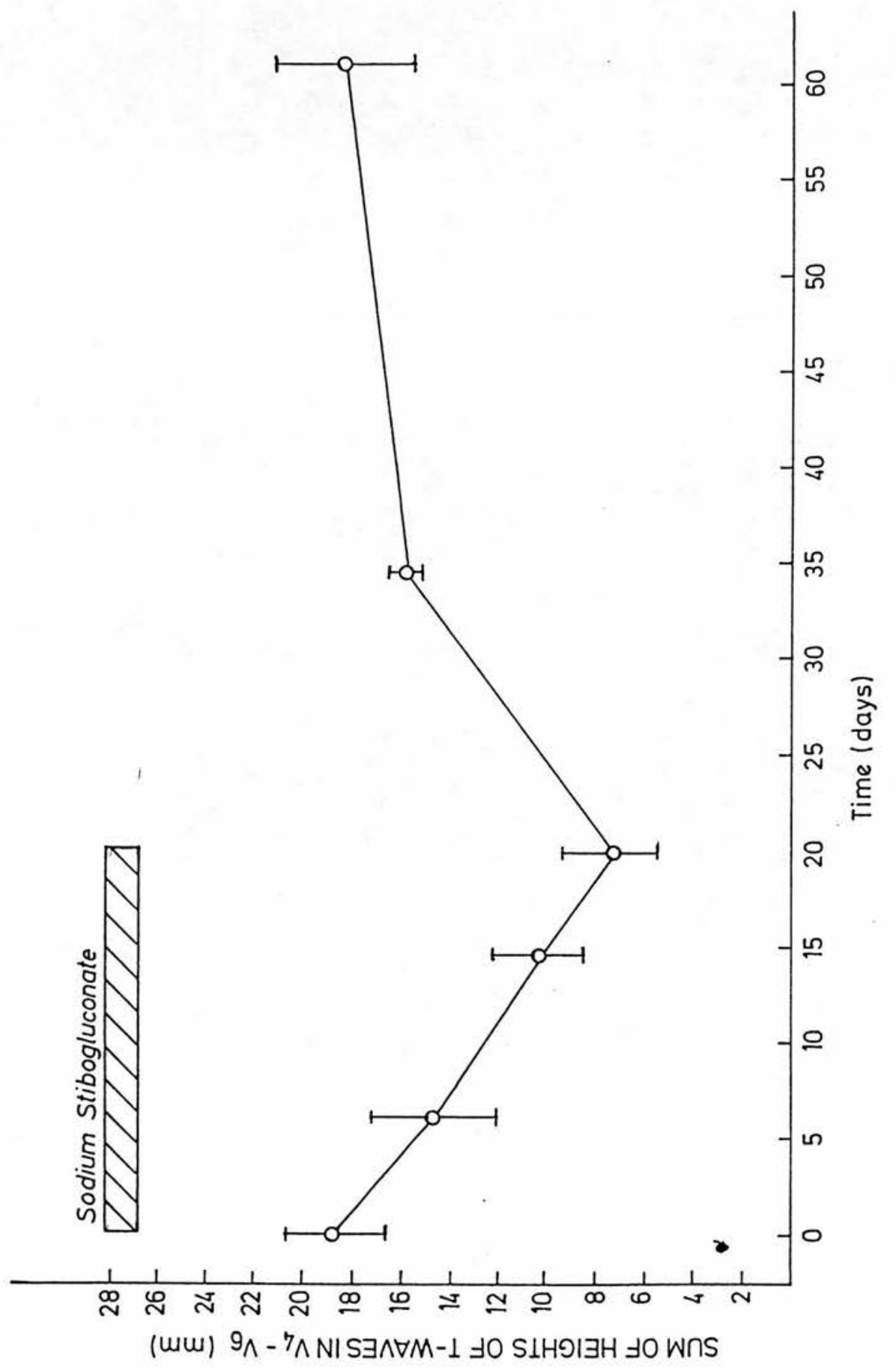


deviation of RR intervals nor the $\mathrm{NN}_{50}$ count changed significantly during the course of this study (table 4.3).

Left ventricular function. There were no significant changes in left ventricular cavity dimensions or measures of left ventricular systolic function (table 4.4). Similarly, the indices of early and late left ventricular diastolic function did not change with treatment (table 4.5).

\subsection{Discussion}

The pentavalent antimonials, sodium stibogluconate and meglumine antimoniate, remain the mainstay of treatment for leishmaniasis. Their precise composition is not known and, indeed, it may vary depending upon the source and batch (Marsden 1985). They contain moieties of very different molecular weights (range 100-4000 daltons) and a variable quantity of trivalent antimony is present (Berman 1988, Marsden 1985). Although sodium stibogluconate is rapidly excreted, largely unchanged, by the kidney, $5 \%$ is excreted as trivalent antimony which has a much longer half-life (Bryceson 1987). The cardiotoxicity of trivalent antimonials is well established (Berman 1988). It was, therefore, important to determine whether the sodium stibogluconate regimen ( $20 \mathrm{mg} / \mathrm{kg} / \mathrm{day}$ for 20 days) currently used to treat American cutaneous leishmaniasis causes cardiac damage.

These results demonstrate that this regime has no measurable effect on left ventricular systolic or diastolic function in fit young adults. There was no biochemical evidence of drug induced myocardial injury during therapy. There was, however, a significant $(p<0.05)$ increase in lactate dehydrogenase when the soldiers had returned to physical duties after completing treatment. This remains unexplained. In the larger study reported in chapter 5 a significant rise in lactate dehydrogenase was not detected. A significant $(p<0.05)$ increase in creatine kinase 6 weeks after the completion of therapy was also noted, but this was entirely due to a large elevation in 2 subjects, one of whom had an elevated heart rate. Although his creatine kinase remained elevated, most was derived from skeletal muscle which may be relevant to the myalgia reported during treatment in all subjects. It was not 
possible to identify either of these subjects when the echocardiographic recordings were examined in a blinded manner by an independent observer (Dr GR Sutherland).

Treatment is associated with a significant, reversible, fall in blood pressure and an increase in heart rate. The cause of these changes is not immediately apparent from our study, but it is likely that they represent a peripheral vasodilatory effect of the drug, since cardiac function remained normal throughout the study in all subjects.

There are, however, antimony-induced effects on the heart. Serial ECGs show a progressive fall in T-wave amplitude during treatment which frequently progresses to $\mathrm{T}$-wave inversion. It returns to normal soon after treatment stops. Similar changes, related to the cumulative dose of antimony administered, have been reported by others (Chulay et al 1985) but this study confirms that these changes occur during treatment with the currently recommended regimen. The trivalent antimonials, tartar emetic and sodium antimonyl tartrate, in therapeutic doses, produce T-wave depression in 60$99 \%$ and prolongation of the QT interval in $9-79 \%$ of patients (Berman 1988). Sodium stibogluconate also produces prolongation of the QTc interval during treatment but only when doses of over $20 \mathrm{mg} / \mathrm{kg} /$ day are used or if treatment is continued for over 30 days (Chulay et al 1985). The ECG changes observed by us and others (Chulay et al 1985) are similar to those described for trivalent antimonials. They are probably due to the accumulation of trivalent antimony during treatment causing changes in ventricular repolarisation. Changes in ventricular repolarisation are known to be associated with an increased risk of ventricular tacharrhythmias (Merx et al 1977) which may be the principle mechanism of sudden death during antimony therapy. Impaired autonomic function (Kleiger et al 1987) and the presence of frequent arrhythmias also increase the risk of sudden death (Multicentre 1983), but neither of these risk factors were present during treatment with sodium stibogluconate in this study. The isolated changes in ventricular repolarisation may not increase the risk of sudden death although a larger study would be need to investigate this relationship in detail. 
In conclusion, the standard treatment regimen for American cutaneous leishmaniasis (sodium stibogluconate $20 \mathrm{mg} / \mathrm{kg} /$ day for 20 days) is not associated with measurable impairment of cardiac function using echocardiographic parameters of systolic and diastolic function. The electrocardiographic changes observed during treatment may be caused by the accumulation of trivalent antimony but they were not associated with electrical instability assessed by arrhythmia analysis and heart-rate variability. However, we cannot exclude more subtle abnormalities or idiosyncratic reactions. 
4.6 Summary: Cardiotoxicity of sodium stibogluconate.

1. The standard treatment regimen for American cutaneous leishmaniasis with sodium stibogluconate $(20 \mathrm{mg} / \mathrm{kg} /$ day for 20 days) is not associated with measurable impairment of cardiac function using echocardiographic parameters.

2. There was a progressive fall in T-wave amplitude during treatment. This is likely to be caused by the accumulation of trivalent antimony causing changes in ventricular repolarisation. However, the ECG changes were not associated with electrical instability assessed by arrhythmia analysis and heart-rate variability. If higher doses of sodium stibogluconate were administered, if the treatment period were prolonged or if renal function were impaired then significant toxicity may be anticipated.

3. There was an unexplained increase in lactate dehydrogenase 6 weeks after treatment when the soldiers had returned to their full physical duties.

4. It is not possible to exclude more subtle abnormalities or idiosyncratic reactions. 


\section{CHAPTER 5}

\section{Aminosidine (paromomycin) versus sodium stibogluconate for the treatment of cutaneous leishmaniasis from Belize.}

5.1 Aims of the investigation

5.2 Introduction

5.3 Patients and methods

5.4 Results

5.5 Discussion

5.6 Summary 


\subsection{Aims of the investigation}

To compare the efficacy of parenteral aminosidine with that of parenteral sodium stibogluconate for treatment of Belizian cutaneous leishmaniasis in British troops.

To compare the adverse effect profile of aminosidine with that of sodium stibogluconate.

To assess the efficacy of sodium stibogluconate $20 \mathrm{mg} / \mathrm{kg} /$ day for 20 days.

\subsection{Introduction}

The treatment for New World CL is unsatisfactory. Sodium stibogluconate is the mainstay of therapy but there are concerns about both its safety and the development of resistance. Its efficacy for the treatment of Belizian CL was confirmed in chapter 2 and it appears to be enhanced if longer courses at a higher daily dose are administered, albeit at the cost of greater morbidity and toxicity. Indeed extrapolation of the results indicated that a single 20 day course of sodium stibogluconate $600 \mathrm{mg}$ twice daily would cure $90 \%$ of cases; such a regimen may offer a reasonable balance between efficacy and toxicity. Although these results are in broad agreement with those obtained for L.panamensis in Panama (Ballou et al 1987), predictions made from data collected retrospectively have many inherent flaws and must be formally tested in a prospective trial. The British Army was unusual in using a fixed daily dose in preference to a dose adjusted for body weight. The WHO and the US Defence Department recommend a dose of $20 \mathrm{mg} / \mathrm{kg} /$ day for New World CL (Berman 1988, WHO 1990).

Sodium stibogluconate is a far from ideal agent, but at the present time there is no highly effective alternative available. Aminosidine, however, has shown early promise; it has anti-leishmanial activity both in vitro and in vivo, it is an effective and well tolerated alternative to sodium stibogluconate for visceral leishmaniasis, when given parenterally, and for CL caused by L.major when used as an ointment (chapter 1). At the start of this study its efficacy in New 
World CL was unknown and therefore an assessment was needed. Recently an ointment containing $15 \%$ aminosidine and $12 \%$ methylbenzethonium chloride shown to heal experimental lesions in mice caused by Belizian strains of both $\mathrm{Lmm}$ and $\mathrm{Lbb}$ (El-On et al 1993). The same ointment healed $74 \%$ of lesions in an open, uncontrolled study in Belize. (Weinrauch et al 1993). In my opinion, Belizian CL should be treated systemically because most cases of $\mathrm{CL}$ in that country are due to $\mathrm{Lbb}$ which may cause mucocutaneous disease. Although cases of $\mathrm{MCL}$ are not frequently seen in Belize, a soldier with MCL from the Belizian Defence force, was recently investigated and treated at the Walter Reed Army Medical Center. He had never been out of Belize (Magill, personal communication). The efficacy of systemic aminosidine in American CL was unknown. The objective of this study was to determine whether or not parenteral aminosidine and optimal sodium stibogluconate were of similar efficacy and whether or not aminosidine offered advantages in terms of toxicity and patient tolerance. This was a preliminary, randomised, open trial conducted entirely by a single observer. A large, blind trial was planned if the results were encouraging.

\subsection{Patients and Methods}

The study was open, randomised and prospective. It was carried out at the army medical facility in Edinburgh and I performed all the clinical observations, took all the samples and administered almost all the treatments personally. It was approved by the Army Medical Services Research and Ethics Committee and all the patients gave their written informed consent (appendix 4.1). All the patients were British soldiers who had contracted CL in Belize and whom had not received any anti-leishmanial therapy for at least 3 months. A full medical history was taken and a general physical examination was performed. Detailed records were made and these included the age and weight of each soldier, the dates of their tour of duty in Belize, and the appearance, duration, size and site of each lesion. If more than one lesion was present then details of each lesion were obtained, but a representative one was selected as the study lesion and all procedures and measurements referred to that lesion. 
Figure 5.1:

Trial protocol.

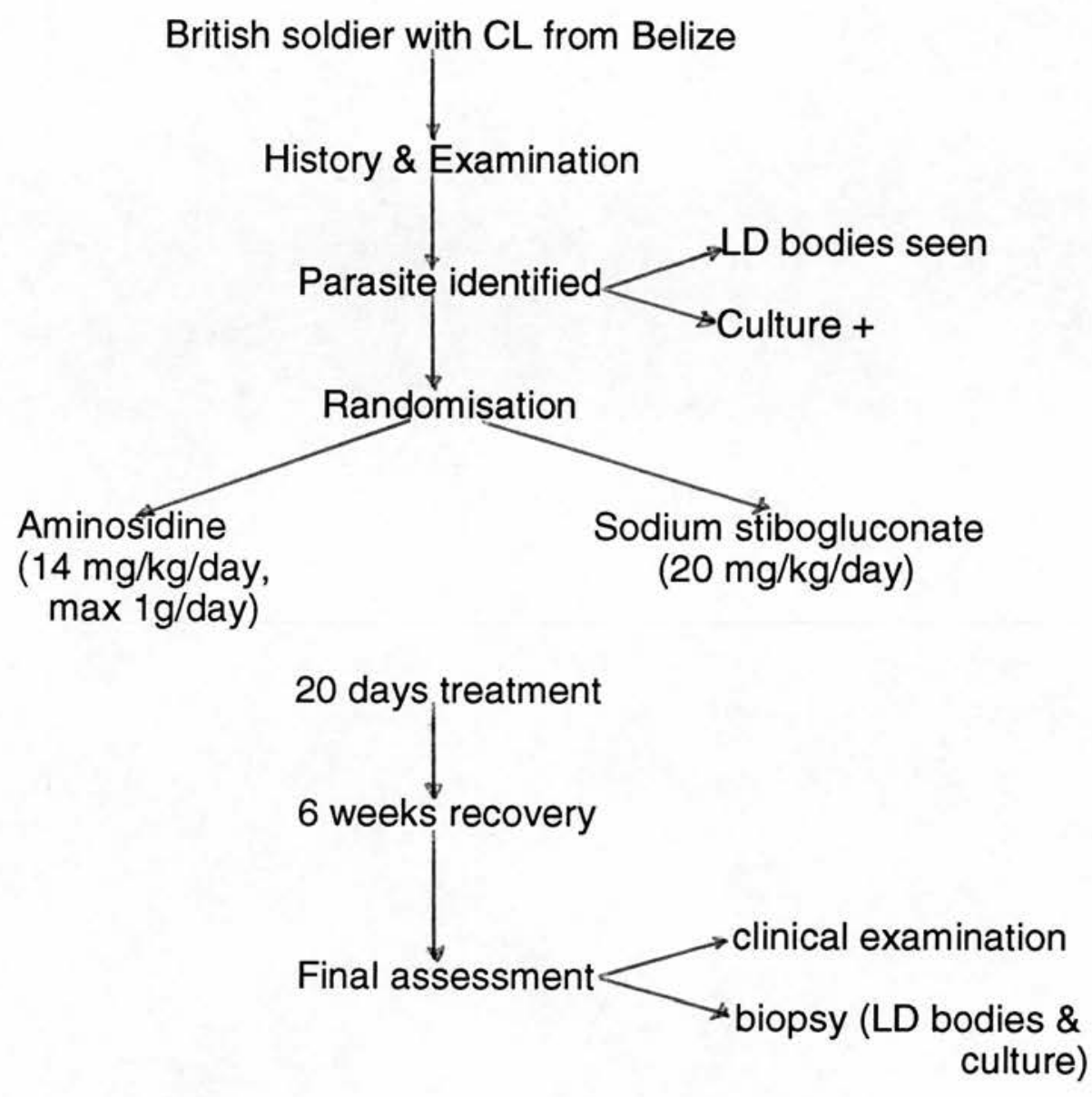

Monitoring:

assessment of lesion

physical examination

FBC

U \& Es, creatinine

Before treatment

LFTs

weekly during treatment

cardiac enzymes

$2 \& 6$ weeks after treatment

ECG

urinalysis

audiogram - before \& after treatment 
An elliptical, full thickness, skin biopsy was taken from the indurated edge of the study lesion: the skin was cleaned with $6 \%$ hydrogen peroxide and then infiltrated with $2 \%$ lignocaine ( \pm adrenaline according to the site), the defect was closed with adhesive strips. Each specimen was divided into 3 parts for histological examination, impression smear and culture. Impression smears were prepared by gently pressing the tissue against a clean, dry microscope slide 4 to 5 times, following which the slide was air dried and then fixed in $95 \%$ ethanol for 3 minutes. The slides were then stained with H\&E and examined for the presence of LD bodies. The specimens for culture were inoculated into "sloppy Evans" media, with added gentamicin, and dispatched to Dr. D. Evans for incubation. If a culture was established, the isolate was typed by isoenzyme analysis (Evans 1989). Specimens for histological examination were formalin-fixed and paraffin-embedded. Sections were stained with H\&E and examined for the presence of LD bodies.

The clinical diagnosis was confirmed by identifying LD bodies histologically and/or by culture in every case before entry to the study. If necessary, the diagnostic procedures were repeated.

Soldiers were randomly allocated (using a computer generated random allocation list supplied by Dr. Drake, Farmitalia Carlo Erba, UK) to receive either aminosidine $14 \mathrm{mg} / \mathrm{kg} /$ day (max $1 \mathrm{~g}$ daily) (Gabbromicina, Farmitalia Carlo Erba, Italy, batch N1004 manufactured Oct 1991) or sodium stibogluconate $20 \mathrm{mg} / \mathrm{kg} /$ day (Pentostam, Wellcome, UK., batch A1058A manufactured Jan 1991). The sodium stibogluconate was stored in the dark at below $25^{\circ} \mathrm{C}$, and once opened it was stored at $4^{\circ} \mathrm{C}$ with no bottle used for more than 10 days. Intravenous treatment with each drug was given for 20 days: the aminosidine was diluted in $400 \mathrm{ml}$ normal saline and infused over 3 to 4 hours, the sodium stibogluconate was given undiluted at $1 \mathrm{ml} /$ minute.

Monitoring. I asked each soldier about his general well being and, specifically, about muscle pains, appetite and nausea everyday before treatment was given. A physical examination, including assessment and measurement of the study lesion, pulse and blood pressure recordings, a full blood count (haemoglobin, white cell and platelet count) (Sysmex NE8000, Sysmex, Japan), urea, electrolytes and creatinine, liver function tests (LFTs: 
bilirubin, ALT, AST, GGT, ALK P and albumin) (BM/Hitachi 747, Boehringer Mannheim, Mannheim, Germany), cardiac enzymes (creatine kinase (CK) and lactate dehydrogenase (LDH)) (Monarch, Allied Instrumentation Laboratory, Warrington, UK) urinalysis and an electrocardiogram (Minigraph, Cardiac Recorders London, UK) were performed before treatment, weekly during treatment and again 2 weeks and 6 weeks after treatment finished. An audiogram (CA 850, Amplivox, Kidlington, Oxford, UK) was performed before and at the end of treatment. A further skin biopsy was taken 6 weeks after the end of treatment (figure 5.1).

Assessment of cure. Lesions were considered to have clinically healed when the ulcer had completely re-epithelialised and the scar was flat and nonindurated. Soldiers were followed up for at least 6 months to ensure that the lesion did not reactivate. Parasitological cure was defined as an absence of LD bodies and a negative culture.

Statistical analysis. The difference in cure rates between the 2 treatment groups was evaluated using a Chi-squared test with Yates correction. The monitoring parameters were compared at each time point with the pretreatment baseline using a paired t-test. The analyses were performed using the statistical software 'Minitab' (Minitab INC, State College PA, USA)

\subsection{Results}

Thirty-four soldiers were enrolled into the study: 17 received aminosidine and 17 received sodium stibogluconate. Their characteristics are shown in table 5.1 .

Efficacy. A complete clinical cure, assessed 6 weeks after treatment had stopped, was observed in 10 (59\%) given aminosidine and 15 (88\%) given sodium stibogluconate, table 5.2 . Sodium stibogluconate was not statistically more effective $(0.5>p>0.1)$. No lesion that was considered to have clinically 
Table 5.1

Clinical features of the study soldiers.

\begin{tabular}{|c|c|c|}
\hline & Aminosidine & Sodium Stibogluconate \\
\hline Number of soldiers & 17 & 17 \\
\hline Age (years) & $23.8 \quad(s d 3.6)$ & $23.5 \quad(\mathrm{sd} 3.8)$ \\
\hline Weight (kg) & $72.8 \quad(s d 7.1)$ & $73.3 \quad(s d 6.6)$ \\
\hline Number of lesions & 1.58 (range1-5) & 1.76 (range $1-3$ ) \\
\hline $\begin{array}{l}\text { Age of lesion at start } \\
\text { of treatment (weeks) }\end{array}$ & $15.7 \quad(s d 10.3)$ & $10.9^{a}(\operatorname{sd~5.8)}$ \\
\hline \multicolumn{3}{|l|}{$\begin{array}{l}\text { Size of lesion at start } \\
\text { of treatment }{ }^{b}(\mathrm{~mm})\end{array}$} \\
\hline ulcer & $18.6(\mathrm{sd} 14.5)$ & $11.8 \quad(s d 5.8)$ \\
\hline induration & $28.0 \quad$ (sd 17.9) & $25.4 \quad(s d 13.5)$ \\
\hline
\end{tabular}

Site of study lesion

$\begin{array}{lrr}\text { head \& neck } & 4 & 4 \\ \text { trunk } & 1 & 1 \\ \text { limbs } & 12 & 12\end{array}$

LD bodies identified

17

17

Culture results

$\begin{array}{lc}\mathrm{Lmm} & 3 \\ \mathrm{Lbb} & 9 \\ \text { negative } & 5 \\ & \\ & \text { xcluding one soldier who contracted CL } 4 \text { years earlier } \\ \text { ngest axis }\end{array}$

$\mathrm{a}=$ excluding one soldier who contracted $\mathrm{CL} 4$ years earlier

$\mathrm{b}=$ longest axis 
healed had reactivated during follow up of at least 6 months (mean 11.4, sd 6.1 months) (figure 5.2).

All 7 soldiers whose lesions had not healed with aminosidine were subsequently successfully treated with sodium stibogluconate. One of the 2 lesions which did not fulfill the criteria for healing with sodium stibogluconate had re-epithelialized but the scar was indurated at the 6 week assessment; this soldier did not receive further treatment and his lesion has not reactivated during follow up of 16 months. Another soldier, treated with aminosidine, had two large ulcers on his neck, one of which was the study lesion, and a small papule on his left knee; both the neck lesions healed (and he therefore counted as a cure) but the papule on his knee enlarged and subsequently ulcerated. A biopsy was taken from that lesion 4 months later and LD bodies were identified. He was then given a course of sodium stibogluconate and the lesion healed.

Leishmania were not cultured nor were LD bodies identified 6 weeks after treatment had stopped (ie parasitological cure) from 9 of 17 lesions treated with aminosidine and 10 of 17 lesions treated with sodium stibogluconate (table 5.2).

Toxicity of aminosidine. Aminosidine was generally well tolerated: only 2 adverse clinical events were recorded. One soldier reported myalgia from days 14 to 19 of treatment. Another, who had no previous history of skin problems, developed a widespread itchy papular eruption on day 11 which was unresponsive to treatment with a topical betamethasone 17-valerate, but which resolved 14 days after he stopped treatment on day 18 (figure 5.3).

There was no significant change in any of the monitoring parameters with the exception of a small $(<3 g / l)$, but significant $(p<0.05)$, rise in albumin on days 20,34 and 64 (figure 5.4). In particular there was no significant change in urea or creatinine and urinalysis was consistently negative. There was no change in any of the soldiers' audiograms. The data is shown in appendices 4.2, 4.4 and 4.6 . 
Table 5.2

\section{Results of treatment.}

Clinically healed

$\begin{array}{lll}\text { total } & 10 / 17 & 15 / 17 \\ \text { Lbb } & 3 / 9 & 7 / 7 \\ \text { Lmm } & 3 / 3 & 3 / 3 \\ \text { re negative } & 4 / 5 & 5 / 7\end{array}$

Parasitological curea

$9 / 17$

$10 / 17$

Post-treatment results

$\begin{array}{lll}\text { LD bodies }^{b} & 8 / 17 & 7 / 17 \\ \text { Lbb } & 4 / 9 & 0 / 7 \\ \text { Lmm } & 0 / 3 & 1 / 3\end{array}$

$\mathrm{a}=$ no $\mathrm{LD}$ bodies seen and culture negative.

$b=$ includes both definite LD bodies and basophilic intracellular fragments.

Toxicity of sodium stibogluconate. Sodium stibogluconate was not well tolerated; all the soldiers reported myalgia which started after 12-17 days of treatment and persisted until 2-4 days after the course finished. Symptomatic treatment with indomethacin was only partially effective. Six soldiers reported loss of appetite and 3 reported headaches. One soldier developed an erythematous macular rash after 17 days of treatment which resolved 2 days after treatment stopped (figure 5.5). There was no significant change in pulse, systolic or diastolic blood pressure, or cardiac enzymes. In 2 soldiers there was a large rise in creatine kinase 6 weeks after treatment. A progressive diminution in the T-wave of the ECG was noted as treatment proceeded but no other abnormality was detected. Significant $(p<0.05)$ falls occurred in the WBC count at days 7, 14 and 20, and in the platelet count at days 14 and 20. In one patient the platelet count to $59 \times 10^{9} / \mathrm{L}$ therefore treatment was stopped after 19 days. There was no change in either urea or 
Figure 5.2a: Lesion due to $\mathrm{Lmm}$ before treatment.
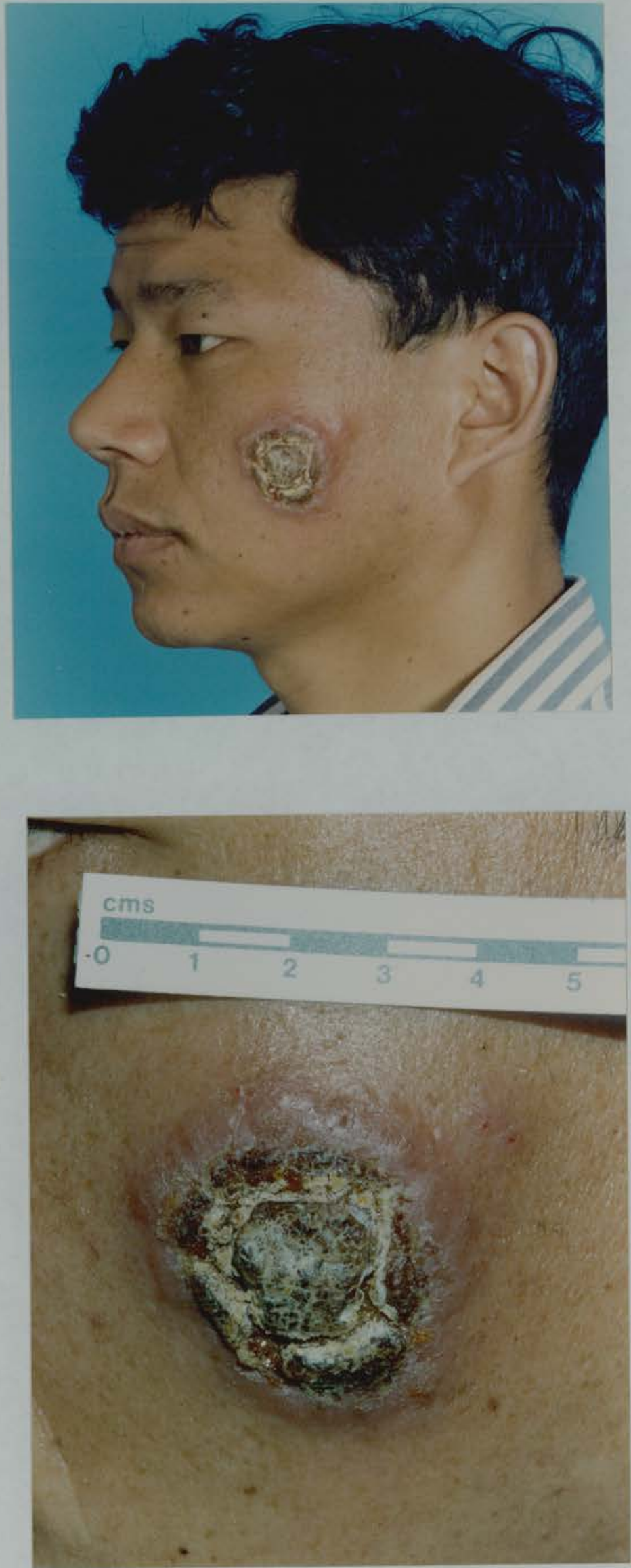
Figure 5.2b: Lesion due to Lmm 6 weeks after treatment with aminosidine.
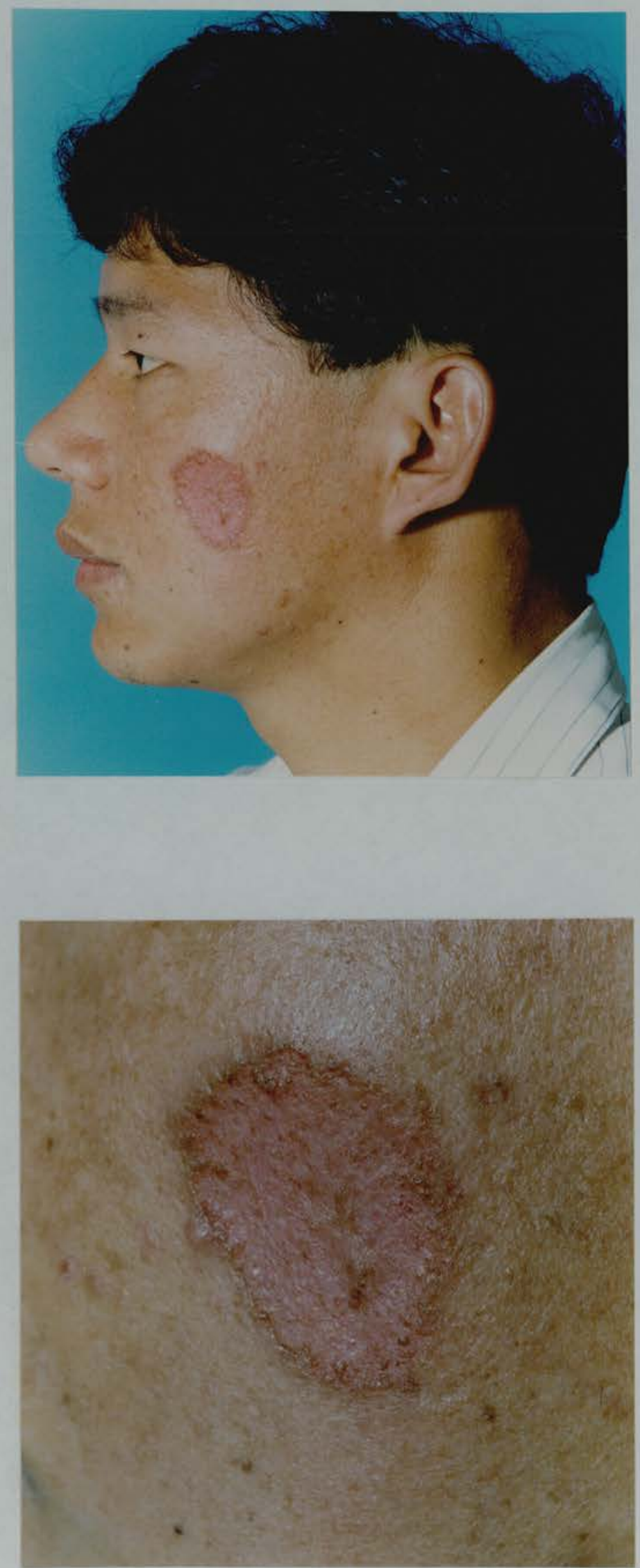
Figure 5.2c: Lesion due to Lmm 1 year after treatment with aminosidine.
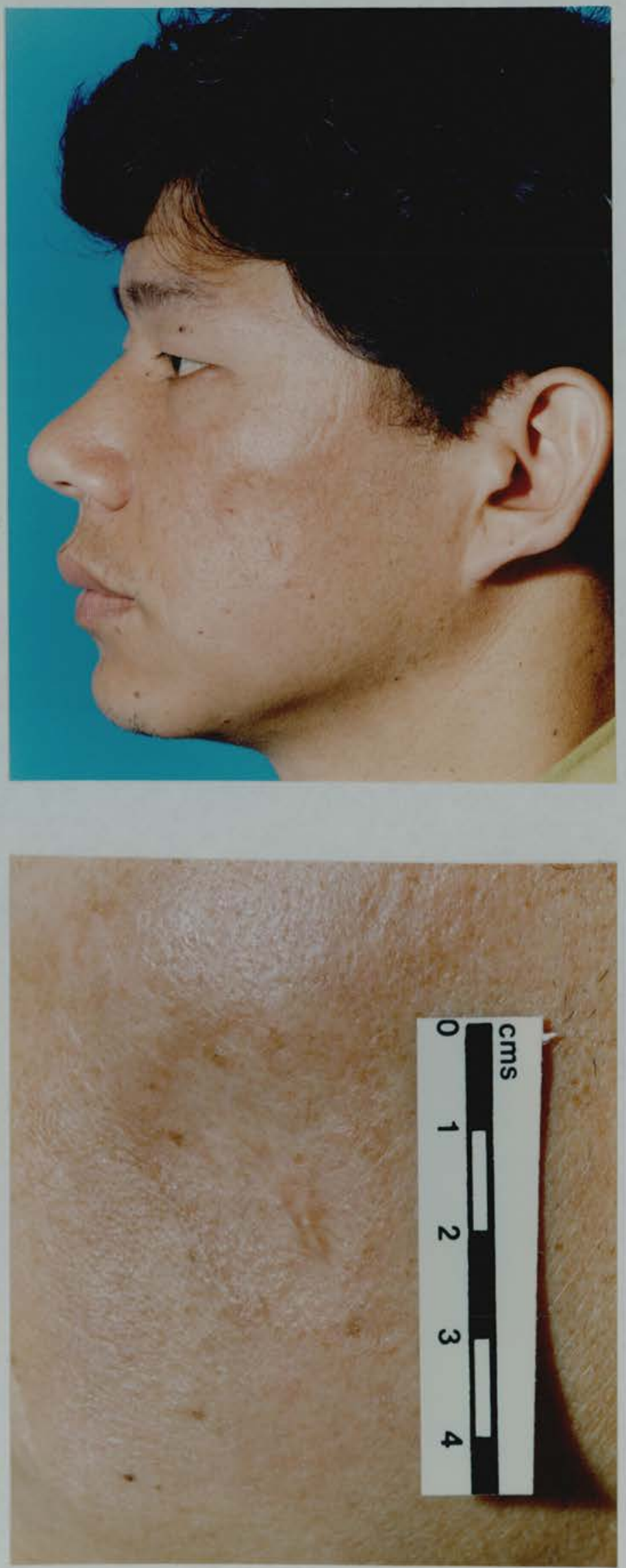
Figure 5.3: Rash during aminosidine treatment.

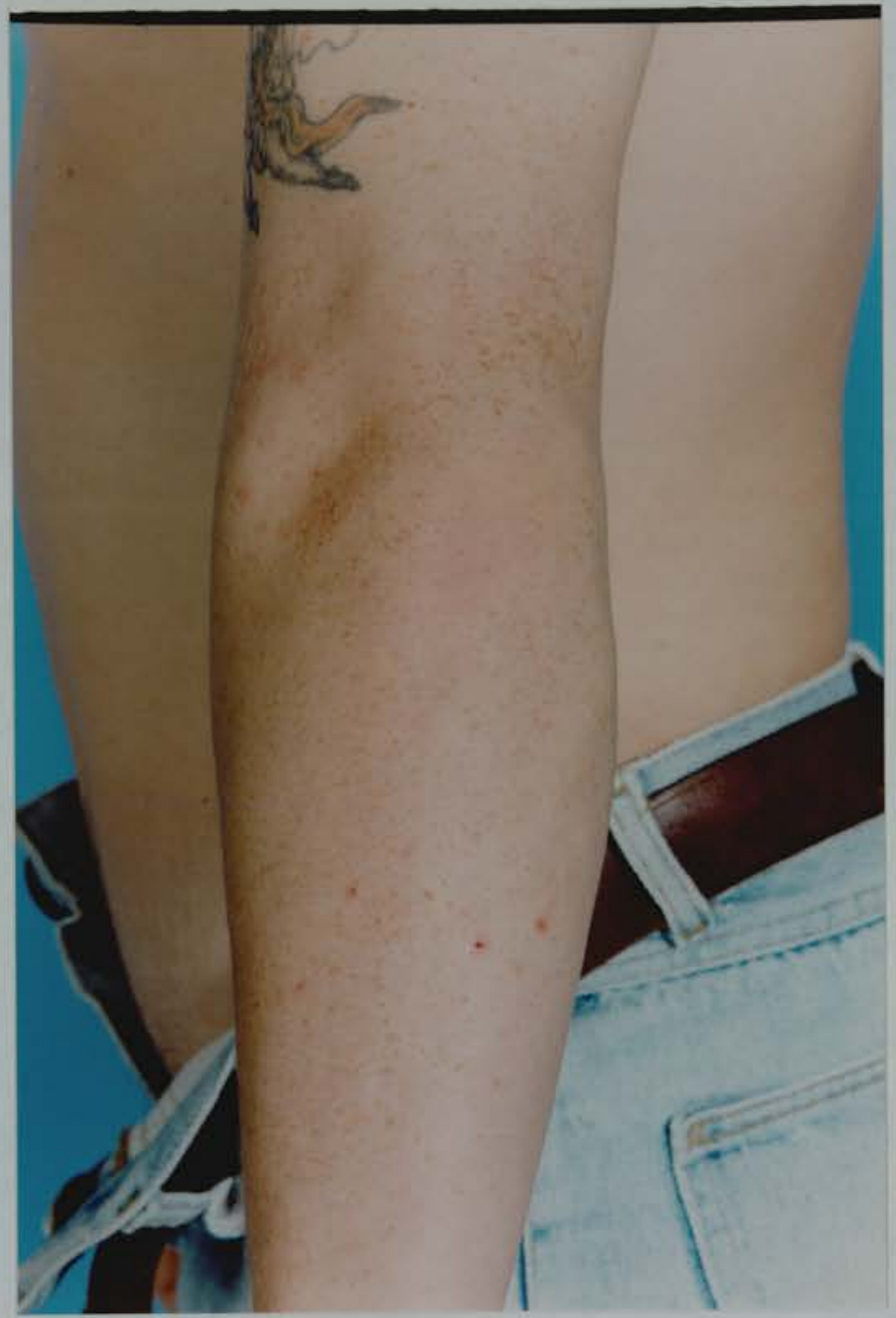


Figure 5.4a: Changes in haemoglogin and white cell count.
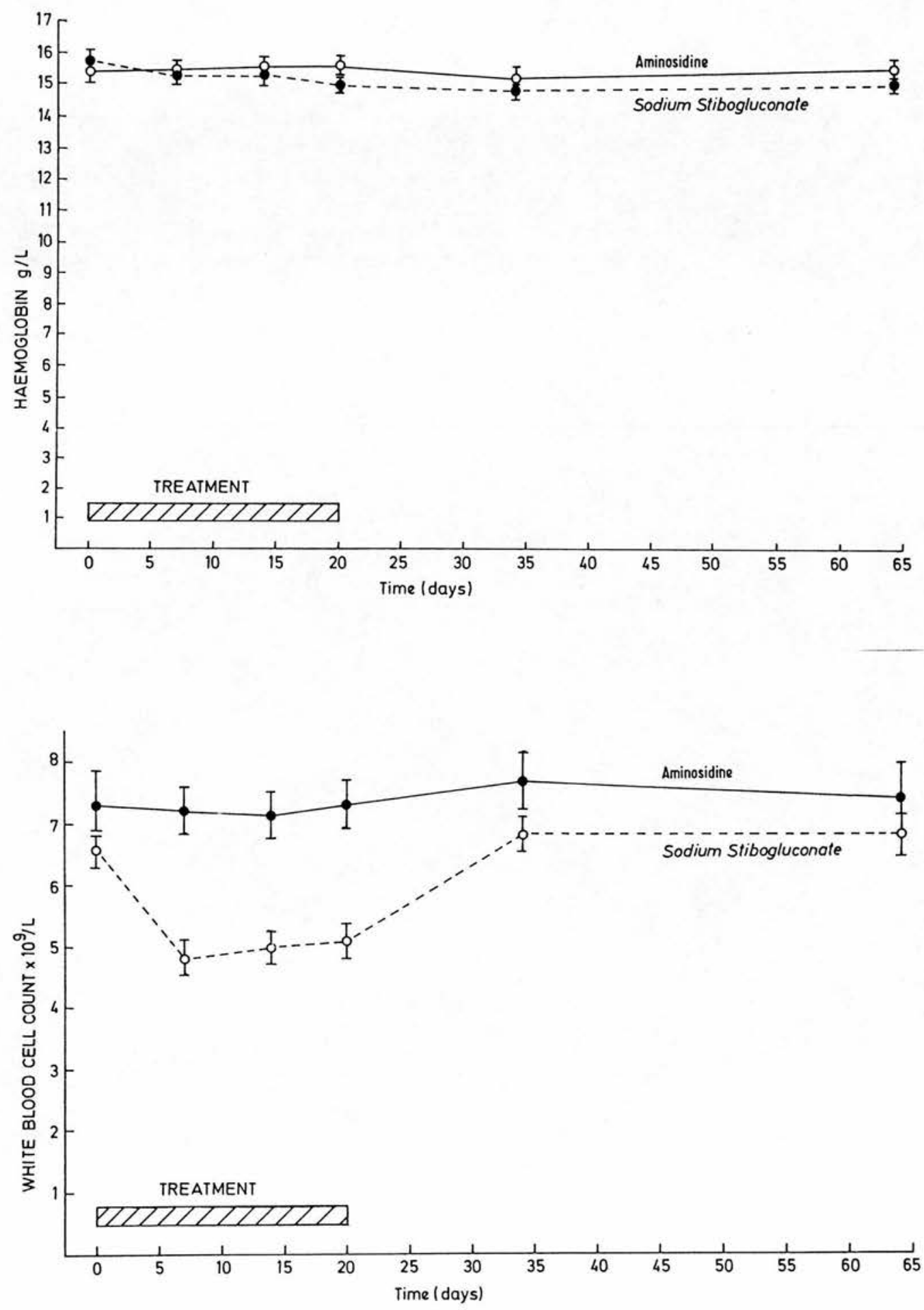
Figure 5.4b: Changes in platelet count and ALT
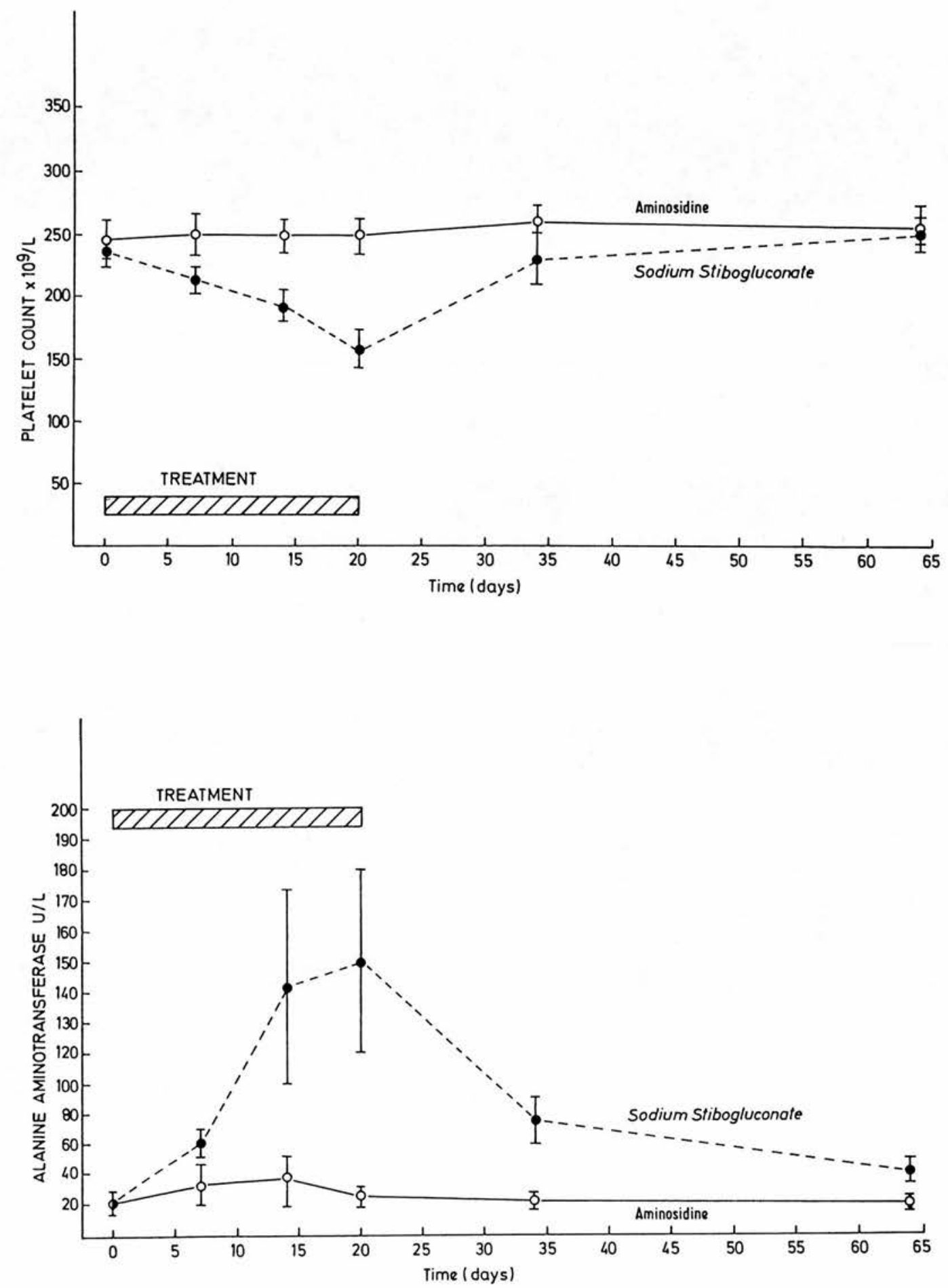
Figure 5.4c: Changes in AST and ALK P.
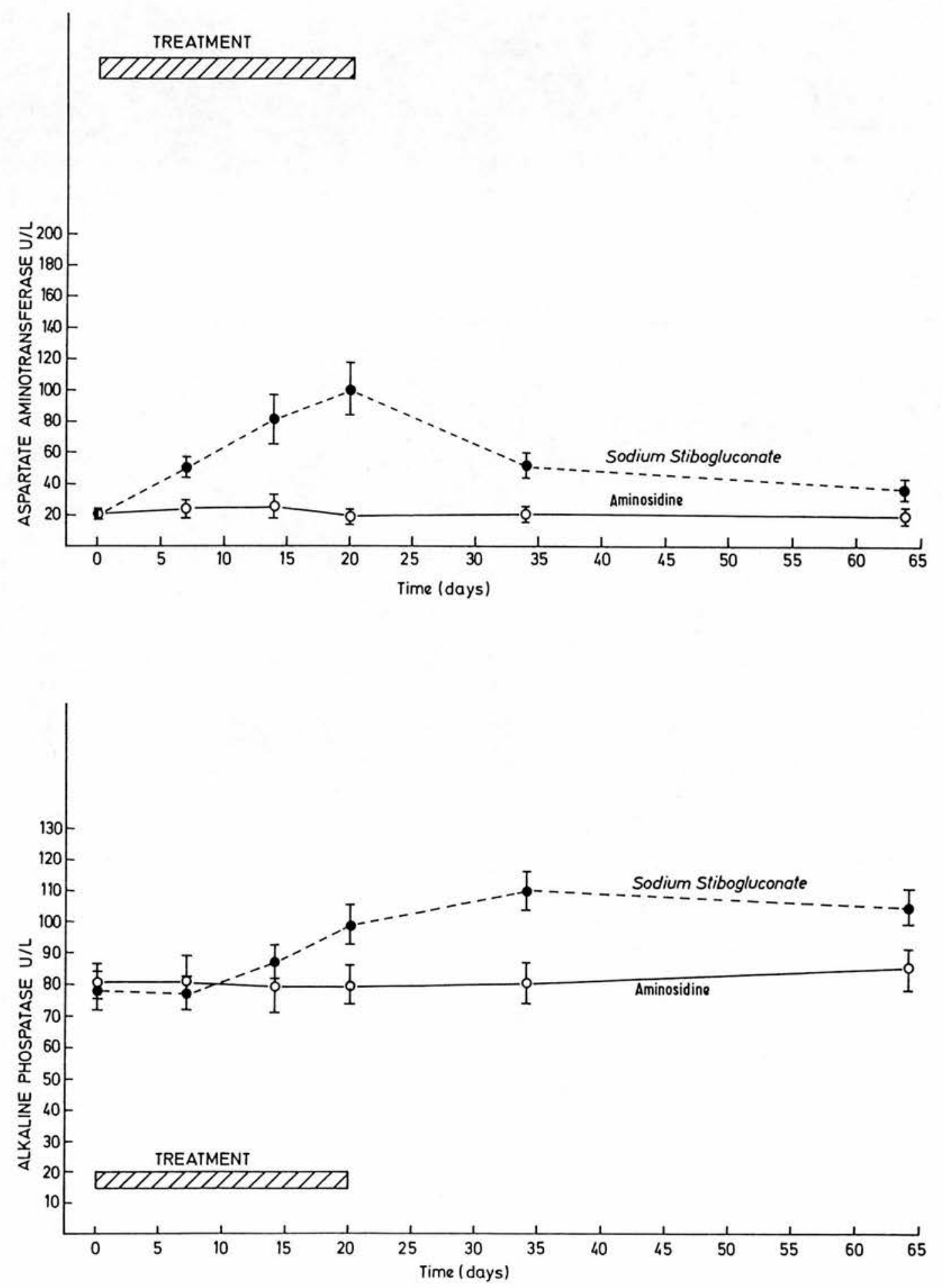
Figure 5.5: Rash during sodium stibogluconate therapy.

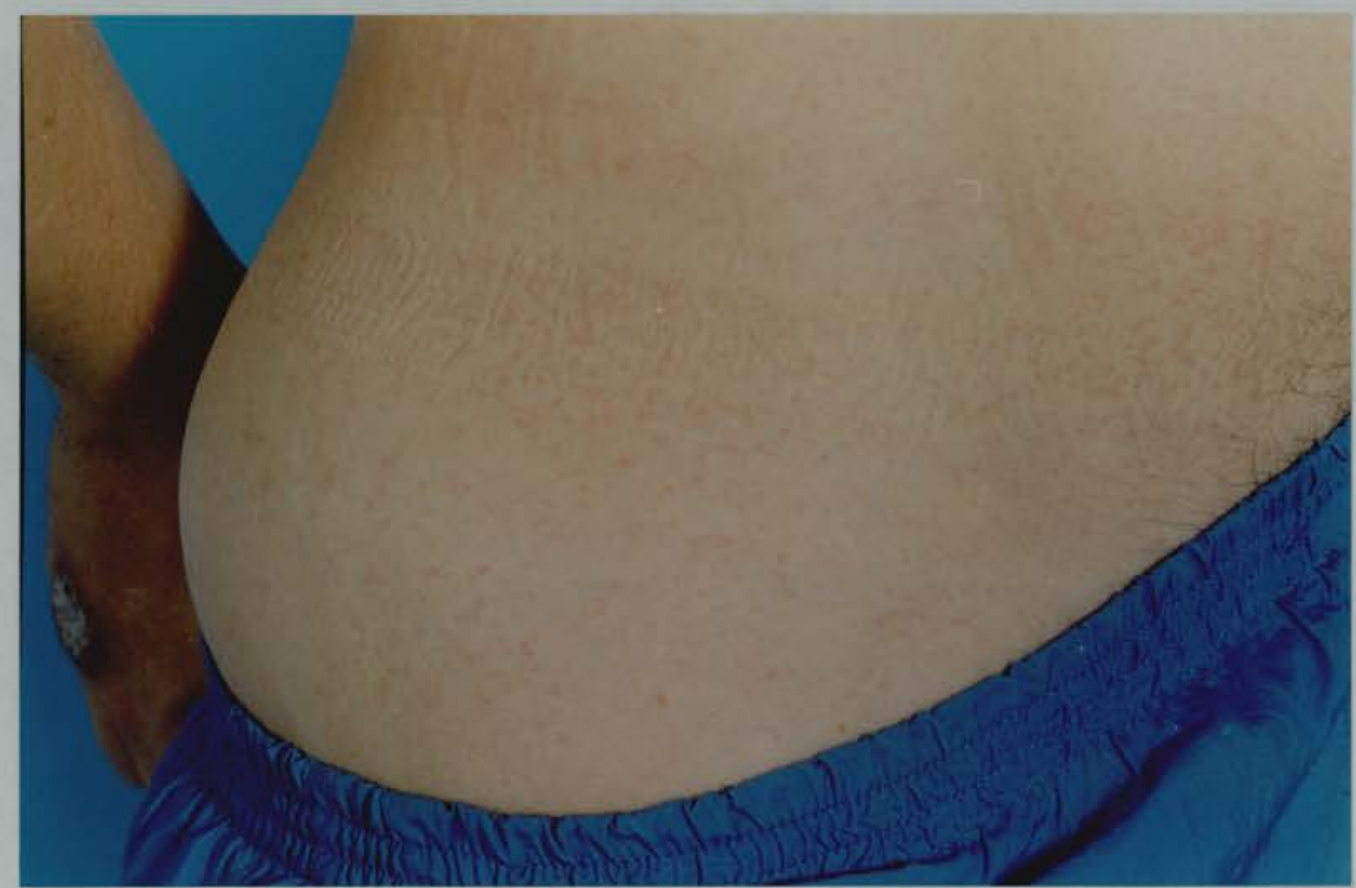


creatinine, and urinalysis was consistently negative. A rise in aminotransferases (ALT and AST) occurred during treatment which, although settling, remained elevated $(p<0.05) 6$ weeks after treatment. A rise in ALK $P$ at days 14 and 20 and 2 weeks after treatment stopped and a small $(<3 \mathrm{~g} / \mathrm{l})$, but significant $(p<0.05)$ fall in serum albumin at the end of treatment and 2 weeks after treatment stopped were also noted. All these parameters had returned to normal when the soldiers were reviewed 3 months after treatment. These changes are shown in figure 5.4. The data are shown in appendices $4.3,4.5$ and 4.6 .

\subsection{Discussion}

The literature on cutaneous leishmaniasis contains numerous reports of 'effective' treatments most of which are based on reports of one or two cases or small, uncontrolled series (Bryceson 1987). Given the self-healing nature of many of the lesions, including those from the New World (Herwaldt et al 1992), it is mandatory to assess any potential treatment in a controlled clinical trial. Ideally there should be a placebo group, to assess the rate of spontaneous healing, and also a group given optimal conventional treatment. However, in Belize most cases of $C L$ are due to $L b b$ which may progress to MCL; this makes the use of a placebo ethically unacceptable. The low rate of spontaneous remission (approximately $6 \%$ of lesions caused by Lbb in neighbouring Guatemala (Herwaldt et al 1992)) would also make a placebo group unacceptable to the army, and to the soldiers, many of whom would be unable to perform their full duties until resolution.

The data from the retrospective study in chapter 2 indicated that a single 20 day course of sodium stibogluconate $600 \mathrm{mg}$ bd would cure approximately $90 \%$ of cases. It is more usual to use a dose adjusted for body weight and the current recommendation is to use $20 \mathrm{mg} / \mathrm{kg} /$ day (Berman 1988, WHO 1990). This has the advantage of avoiding toxicity in smaller soldiers, although for most it represents a greater daily dose (mean weight $73 \mathrm{~kg}$, therefore $600 \mathrm{mg}$ bd $=16.4 \mathrm{mg} / \mathrm{kg} /$ day). A dose of $20 \mathrm{mg} / \mathrm{kg} /$ day for 20 days was, therefore, selected in order to aid comparison and because this regimen had been shown to cure $90 \%$ of lesions caused by L.panamensis from 
Panama (Ballou et al 1987). The results confirmed that it would cure $88 \%$ of lesions from Belize. However, there was considerable morbidity, particularly towards the end of the course of treatment, with all patients reporting muscle pains and many also suffering nausea during the final 7 days. This was coupled with falls in the white blood cell and platelet counts, indicative of bone marrow toxicity, and elevations in the aminotransferase levels, indicative of hepatocellular damage. The haematological abnormalities returned to normal after treatment. The hepatotoxicity has been more fully investigated in chapter 3. Two soldiers developed a rise in creatine kinase 6 weeks after treatment, by which time they had returned to full physical duties. In one this remained elevated 14 months after treatment; at that time only $2 \%$ was CK$M B$ indicating most was der ived from skeletal muscle. In view of the myalgia experienced by the soldiers during treatment further assessment is need 2 . In the first instance the CK and CK-MB should be measured in a large sample of soldiers who have been treated with sodium stibogluconate. Those in whom an abnormality is detected and 2 control groups: (1) those who do not appear to be affected and, (2) normal soldiers who have not received sodium stibogluconate should be studied. Assessment should include creatine kinase levels before and after exercise, electromyography, and a muscle biopsy.

Aminosidine is less efficacious, but better tolerated, than sodium stibogluconate. Although the difference in efficacy did not reach statistical significance in this study, this is almost certainly a reflection of the lack of power of the study rather than the absence of a real difference. A larger study has just been completed in Colombia and the results are similar aminosidine healed $60 \%$ of lesions and sodium stibogluconate healed $95 \%$ of lesions. Unfortunately the species of leishmania was not identified and close monitoring for toxicity was not possible in that study (Berman, personal communication). Aminosidine appears to be relatively inactive against Lbb the most common type of $\mathrm{CL}$ in Belize. It is these cases that are of most importance clinically; not only are they unlikely to heal spontaneously, but they may cause MCL. However, aminosidine has been shown to be efficacious in visceral leishmaniasis and it may prove to be of use in other types of CL. The safety of aminosidine shown by this study, and that in chapter 3 , is encouraging. In this study the aminosidine was administered intravenously over 3-4 hours. Chunge et al (1990) and Thakur et al (1992), 
administered it intramuscularly. Scott et al (1992), gave it intravenously over 100 minutes (to mimic the pharmacokinetic profile of intramuscular absorption) but encountered high tone deafness and impairment of renal function during treatment despite assaying drug levels. I was not able to assay drug levels and high-tone hearing impairment would have had profound consequences for a soldier's career. The regimen used in this study was suggested by Dr. ADM Bryceson and Dr. P. Olliaro who had been involved with the study reported by Scott et al (1992).

The concept of parasitological cure (ie the absence of LD bodies and a negative culture following treatment) was of little use because it was difficult to be confident that intracellular basophilic fragments were not viable leishmania. A post treatment culture alone, which by definition identifies viable organisms, was useful but the small numbers in this study preclude meaningful conclusions. It is possible that leishmania persist in healed lesions. Cure usually results in life-long protection from the disease which is mediated by the cell-mediated immune response; this in turn could be maintained by the continuous antigenic stimulus provided by the persistence of a few live parasites (Aebischer 1994). A study of recurrent disease caused by L.braziliensis subspecies in Colombia showed that in $50 \%$ of cases parasites cultured from secondary or tertiary lesions were indistinguishable by 3 independent methods (zymodeme, schizodeme and karyotype analyses) from the leishmania isolated during the primary infection. Futhermore, secondary lesions caused by the same parasites were usually close to the site of the original lesion, whereas those caused by different parasites were usually located elsewhere (Saravia et al 1990). If viable leishmania do persist this may have important consequences if the patients subsequently become immunosupressed. Relapse, induced by immunosuppression, after a 'cure' has been demonstrated in mice (de Rossell et al 1992).

In conclusion, aminosidine is not as efficacious as sodium stibogluconate for the treatment of $\mathrm{CL}$ from Belize, particularly in those cases due to Lbb. It is, however, safe and well tolerated; if its efficacy in other forms of leishmaniasis is established then it may have a place in the therapy against those forms of leishmaniasis. The cure rate attained with a single course of sodium stibogluconate can be increased to approximately $90 \%$ as predicted in the 
retrospective study in chapter 2 . However, there is a considerable increase in toxicity, which, although reversible, may occasionally be serious and require intervention; careful monitoring is therefore essential. 
5.6 Summary: Aminosidine (paromomycin) versus sodium stibogluconate in the treatment of cutaneous leishmaniasis from Belize.

1. Aminosidine is less efficacious than optimal sodium stibogluconate for the treatment of Belizian CL.

2. Aminosidine is relatively inactive against $\mathrm{Lbb}$ which is the most important cause of Belizian CL.

3. Aminosidine is well-tolerated and safe.

4. A single course of sodium stibogluconate $20 \mathrm{mg} / \mathrm{kg} /$ day for 20 days will heal $88 \%$ of lesions.

5. This intensive regimen of sodium stibogluconate is associated with more morbidity and toxicity, particularly thrombocytopenia, leucopenia and hepatocellular damage, than the previously used low dose regimens. Careful monitoring during treatment is, therefore, required. 


\section{Chapter 6}

\section{The diagnostic Pathology of Belizian Cutaneous Leishmaniasis.}

6.1 Aims of the investigation

6.2 Introduction

6.3 Methods

6.4 Results

6.5 Discussion

6.6 Summary 


\subsection{Aims of the Investigation.}

To describe the pathological changes observed.

To assess the value of impression smears in diagnosis.

To assess the value of routine haematoxylin and eosin (H\&E) versus Giemsa staining of biopsy material.

To assess the value of a post-treatment biopsy in patient management.

To make a preliminary assessment of the value of immunocytochemistry as a diagnostic aid.

\subsection{Introduction}

American $\mathrm{CL}$ is usually diagnosed clinically on the basis of a typical lesion in conjunction with an appropriate history of exposure, but, because the differential diagnosis is extensive and treatment for most patients involves the relatively toxic pentavalent antimonials, it should be confirmed, ideally by demonstrating the parasite. Unfortunately, no single test is optimal. It is therefore usual to perform several, such as histology, impression smears, culture, serology, and/or a Montenegro test, depending upon the facilities available locally.

Belizian cutaneous leishmaniasis can be caused by either $\mathrm{Lmm}$ or, more commonly, Lbb. The lesions are clinically indistinguishable and must be differentiated by culture and isoenzyme analysis (chapter 2 ). Histological examination of the lesion is the most widely available method of investigation, but the number of leishmania present falls with the passage of time and they are reputed to be particularly scanty in lesions due to Lbb (Gutierrez et al 1991). In the absence of leishmania the histology may resemble that of syphilis, tuberculosis and sarcoidosis (Lucas \& Kirkham 1991), although the nature of the inflammatory reaction pattern should allow a probable diagnosis to be reached. Lesions caused by Lmm may heal spontaneously whereas 
those due to Lbb are unlikely to do so (Herwaldt at al 1992), added to which there is the danger of late mucocutaneous disease. Hence, it is especially in those patients with $\mathrm{Lbb}$, in whom the diagnosis is most important, that histological confirmation proves most difficult. The retrospective review (chapter 2) revealed that LD bodies had been identified in only 141 of 306 cases (46\%), although histological examination had not always been performed. Although culture may be more sensitive than histology (Lucas, personal communication), it is not so widely available and the delay in obtaining a definitive result means that treatment is generally started before the result is available. This is a particular problem for the army because soldiers are often unable to perform their full duties until their lesion has healed. A rapid, reliable, diagnostic test would therefore be of great value, as would a method of improving the sensitivity of histological assessment. Giemsa staining of biopsy material is reputed to be superior to the routinely used $\mathrm{H} \& \mathrm{E}$ stain because the parasites are stained red whereas they appear blue-grey with H\&E (McKee 1989). Impression smears have been reported to be a useful rapid diagnostic test (Berger et al 1987). The value of immunocytochemistry is disputed (Lucas \& Kirkham 1991, Salinas at al 1990).

A further problem is the assessment of cure. In routine clinical practice lesions are considered to have healed when they have re-epithelialised and the scar is flat and non-indurated. However, small numbers of viable parasites could remain and may lead to subsequent reactivation of the lesion. Alternatively, lesions in which all the parasites have been killed may be slow to reepithelialise and further, unnecessary, treatment may be given. It is not known whether a biopsy taken after treatment assists in patient management.

In order to address these issues, I undertook a collaborative study with a pathologist, Dr L Biddlestone and we examined the sections together.

\subsection{Methods}

Patients. British soldiers who had contracted CL whilst on duty in Belize were treated at the army medical facility in Edinburgh as part of a prospective, randomised study to assess the efficacy of aminosidine versus sodium stibogluconate, which is fully described in chapter 5 . Skin biopsies were 
taken from the indurated edge of the lesions before treatment and each specimen was divided into 3 parts for histological examination, impression smear and culture.

Six weeks after the completion of treatment soldiers were reassessed, lesions were considered to have healed clinically if the ulcer had completely reepithelilized and the scar was flat and non-indurated. A second biopsy was taken at that visit for histology from every lesion and for culture in 31 of the 34 lesions (the specimens were lost in transit in the remaining 3 cases).

Impression smears were prepared by gently pressing the tissue against a clean, dry microscope slide 4 to 5 times following which the slide was air dried and then fixed in $95 \%$ ethanol for 3 minutes. The slides were then stained with $\mathrm{H} \& \mathrm{E}$ and examined for the presence of amastigotes.

Culture. Specimens were inoculated into "sloppy Evans" media, with added gentamicin, and dispatched for incubation. If a culture was established the isolate was typed by isoenzyme analysis (Evans 1989).

Histology. The skin biopsies were formalin-fixed, routinely processed, and paraffin-embedded. Sections were stained with H\&E and 11 biopsies were also stained with Giemsa. Histological assessment included: (1) a semiquantitative assessment of parasite numbers (table 6.1), (2) the extent and nature of the inflammatory cell infiltrate including granulomas, (3) the presence or absence of dermal necrosis or fibrosis, (4) assessment of the epidermal changes, and (5) an attempted classification using (i) the general histological groupings described by Ridley (1987) and, (ii) a specific classification developed for Brazilian CL (Ridley et al 1980).

Immunocytochemistry. In a preliminary study, a polyclonal antibody, raised in rabbits against $\mathrm{Lmm}$ promastigotes (kindly gifted by $\mathrm{Dr} \mathrm{J}$ Alexander, Glasgow), was used to identify organisms in formalin-fixed sections. A standard avidin biotin technique was used to stain 4 biopsies from lesions which were culture positive and which contained easily identifiable LD bodies on the H\&E sections. Three biopsies contained Lmm and one Lbb. 


\subsection{Results}

Clinical and culture. Thirty-six pre-treatment biopsies from 34 soldiers, mean age 23.6 ( $s d$ 3.6) years, were taken. In 2 cases the same lesion was sampled twice. The lesions had been present for a mean of 14.6 (sd 8.3) weeks when the biopsy was taken. All but 2 of the lesions were ulcers (mean diameter 15 (sd 10) $\mathrm{mm}$ ), usually with an indurated margin; one was a plaque and the other a papule surmounted by a crust.

Culture was established from 23 lesions, Lbb was identified in 16 and $\mathrm{Lmm}$ in 7. Histologically well formed LD bodies were seen in 16 of the culture positive specimens ( $10 \mathrm{Lbb}$ and $6 \mathrm{Lmm}$ ) but it was only possible to identify fragments of basophilic material in the remainder (table 6.1).

A total of 9 lesions failed to heal 6 weeks after treatment; in 6 the ulcer never re-epithelialized and in the others the scar remained indurated. None of the 25 lesions that healed has reactivated during follow up of at least 4 months (range 4 - 24 months). Leishmania were cultured from 5 lesions after treatment ( $4 \mathrm{Lbb}$ and $1 \mathrm{Lmm}$ ); the ulcers persisted in 2, induration was the only sign in a further 2 and the remaining lesion (due to $\mathrm{Lmm}$ ) had completely healed clinically (and it has remained healed for over a year without further treatment). Eight of the 9 cases which failed to heal were successfully retreated with sodium stibogluconate (in the remaining case the scar was indurated at the assessment 6 weeks after treatment; this settled without further treatment). Five were re-biopsied and culture was negative.

Histology. All 36 pre-treatment biopsies had a similar appearance with a moderate to heavy dermal infiltrate including lymphocytes, plasma cells and macrophages (figure 6.1). Lymphocytes were the predominant cell type in 21, plasma cells in 11 and macrophages in 4 biopsies. Granulomatous inflammation was a prominent feature. In 30 biopsies palisading around foci of necrosis or degenerate collagen was seen. In 11 biopsies well-formed epithelioid cell granulomas with giant cells and a rim of lymphocytes were present (figure 6.2). In 33 biopsies there was a variable degree of dermal connective tissue change with collagen degeneration, small foci of necrosis or larger confluent areas of dermal necrosis. The majority of untreated lesions 
exhibited notable epidermal changes with florid epithelial hyperplasia in 35 and epidermal ulceration in 29 biopsies. An acute inflammatory cell infiltrate accompanied this ulceration and neutrophils were also seen around the areas of dermal necrosis. Parasites were typically scanty. They were usually found within the clear cytoplasm of macrophages, often in the superficial part of the lesion. Obvious parasites were identified in 20 biopsies and were numerous in 7 biopsies (figure 6.3). In some biopsies parasites with a classical morphology were not identified, although intracellular basophilic fragments were present which may have represented degenerate forms. H\&E stained sections were superior to Giemsa stained sections for identification of the parasites. Histological examination and culture of biopsy material were complementary techniques for identification of the organisms with LD bodies definitively identified in 4 culture negative cases (table 6.1).

\section{Table 6.1}

Semi-quantitative organism counts in pre-treatment biopsies.

\begin{tabular}{llllllll}
\hline \multicolumn{1}{c}{ Histological Scores } \\
Culture & $0+$ & $1+$ & $2+$ & $3+$ & $4+$ & $5+$ & \\
Lmm & 1 & 2 & 0 & 2 & 2 & 0 & $(n=7)$ \\
Lbb & 6 & 7 & 0 & 2 & 1 & 0 & $(n=16)$ \\
Negative & 9 & 3 & 1 & 0 & 0 & 0 & $(n=13)$ \\
\hline
\end{tabular}

Key: $0+=$ no well-defined organisms, fragments only.

$1+=$ small numbers of LD bodies focally.

$2+=$ small numbers of LD bodies throughout the lesion.

$3+=$ moderate/numerous LD bodies focally.

$4+=$ numerous LD bodies in most parts of the lesion

$5+=$ very numerous LD bodies in all parts of the lesion.

Post-treatment biopsies were taken from the 25 clinically healed lesions 6 weeks after treatment and within 8 weeks of re-epithelialisation. All showed a marked reduction in the inflammatory response (figure 6.5). The residual infiltrate consisted of perivascular lymphocytes and 9 of the 25 biopsies 
showed well-formed dermal granulomas which included epithelioid macrophages and giant cells. In contrast to the pre-treatment biopsies, dermal necrosis was uncommon and the main change seen in the dermis was fibrosis. The previously marked epidermal changes had also regressed; in 12 there was no residual abnormality and in the remainder there was only mild acanthosis. Definite parasites were only very occasionally observed in these biopsies but intracellular basophilic fragments were frequently present.

Post-treatment biopsies from lesions which had failed to heal after the first course of treatment showed little change from their pre-treatment appearance. The moderate to heavy inflammatory infiltrate persisted and in 7 dermal necrosis was still present although fibrosis was also seen. Moderate numbers of parasites had been identified before treatment in 4 of these lesions and they were still present in 2; in one the number of parasites identified in the sections had increased. Biopsies following a second, successful course of treatment were available in 5 cases. They were indistinguishable from the lesions which had responded to the first course of treatment. There were no histological features in the pre-treatment biopsies of this group of cases to distinguish them from the majority which responded to the first course of treatment.

Impression smears. Parasites were often difficult to identify in the histological sections and the smears were even more difficult to interpret (figure 6.6). In no case were definite parasites identified in the smears when the histological sections were equivocal.

Immunohistochemistry. Immunocytochemical staining of sections from 3 biopsies from which $\mathrm{Lmm}$ had been cultured gave specific, strong staining of LD bodies with low background staining (figure 6.4). However, in this preliminary study, only biopsies with readily identifiable parasites were used. One biopsy, from which Lbb had been cultured, was negative even though definite organisms were easily seen on the H\&E sections. 
Figure 6.1: Pre-treatment biopsy from a lesion caused by Lmm stained with H\&E.

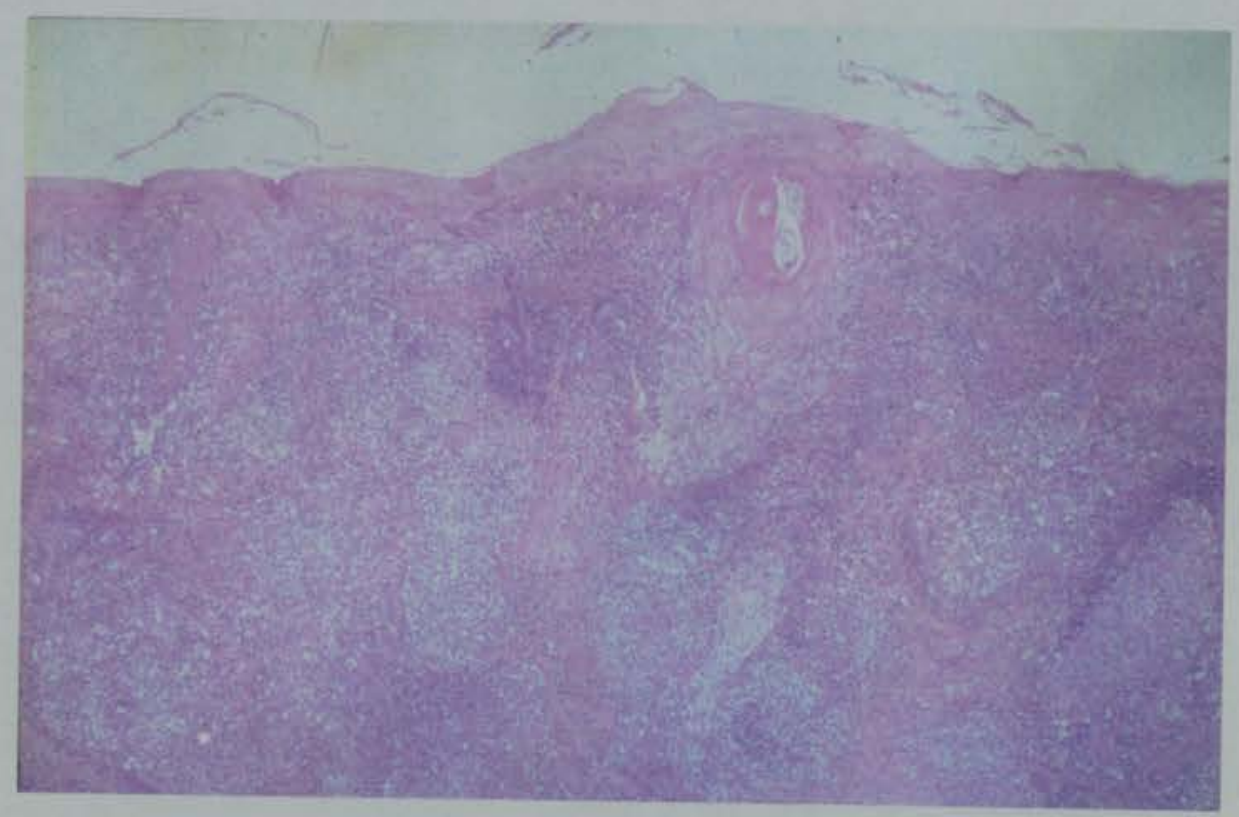

Figure 6.2: Dermal inflammatory infiltrate showing plasma cells and giant cells. Lesion caused by Lbb.

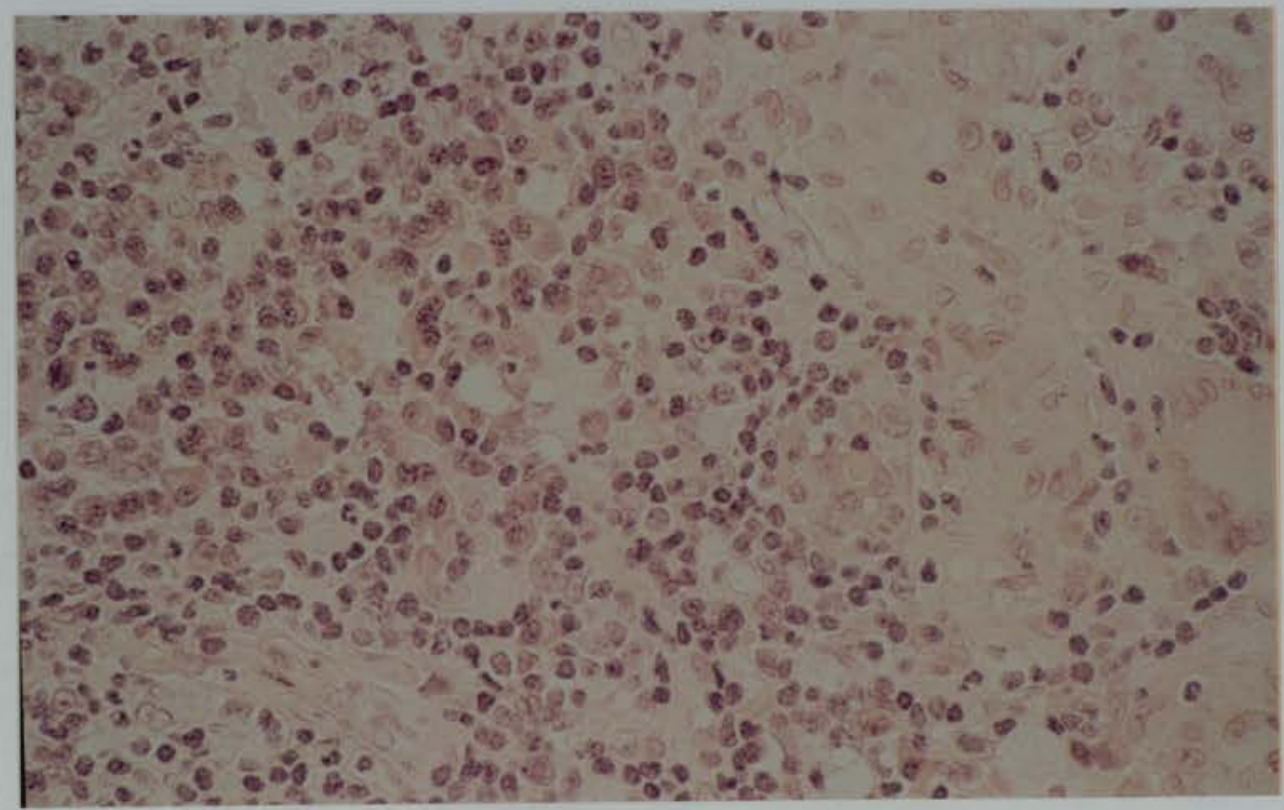


Figure 6.3: Numerous LD bodies in the dermis in a lesion caused by Lmm.

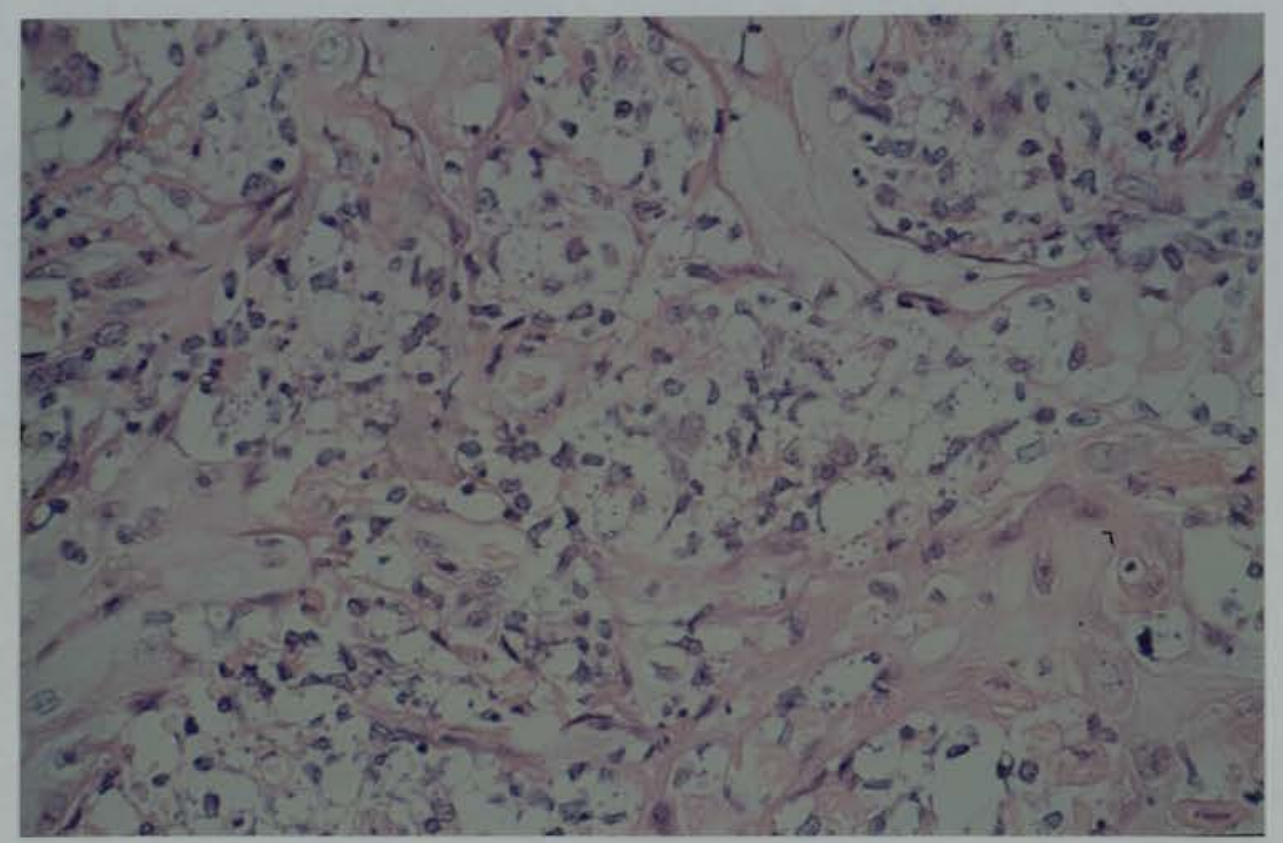

Figure 6.4: Biopsy shown in figure 6.3 labelled with anti-Lmm polyclonal antibody and stained by immunoperoxidase.

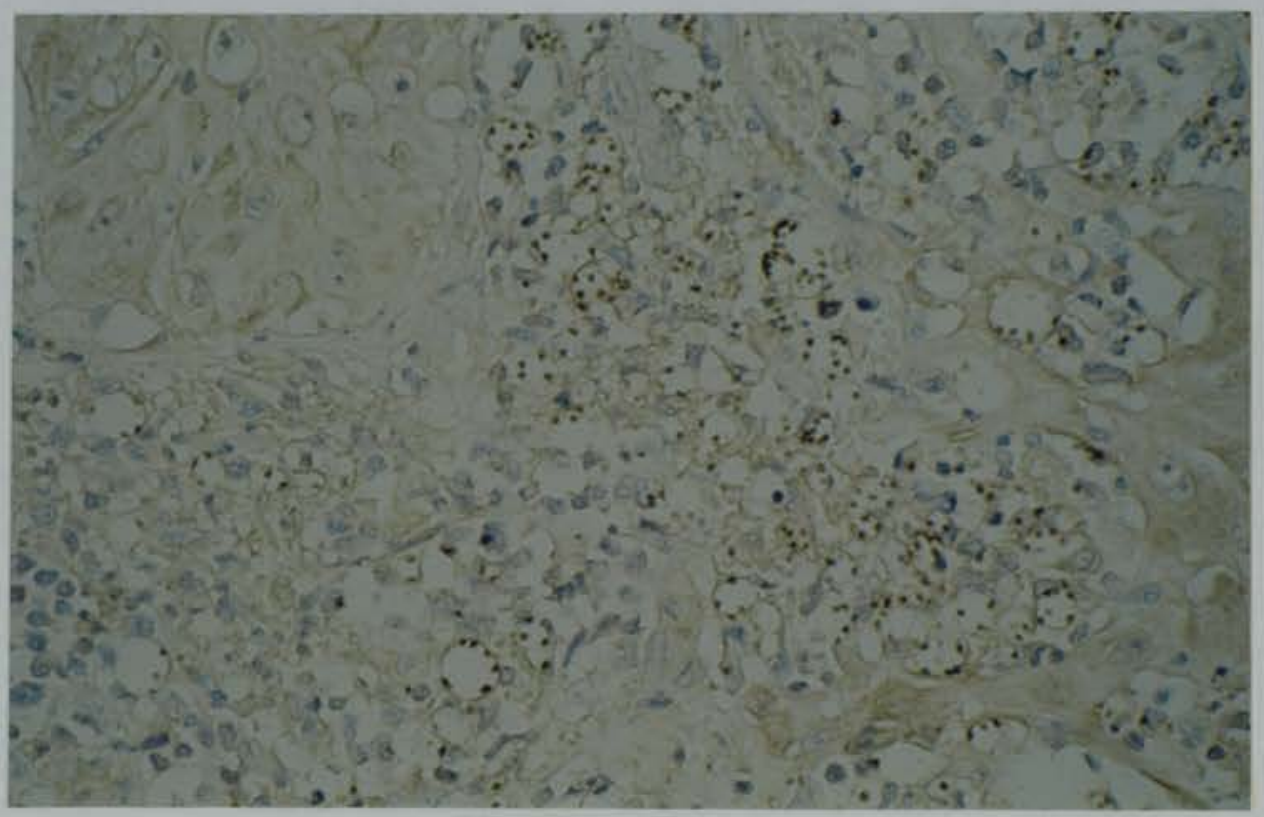


Figure 6.5: Lesion caused by Lbb 6 weeks after successsful treatment.

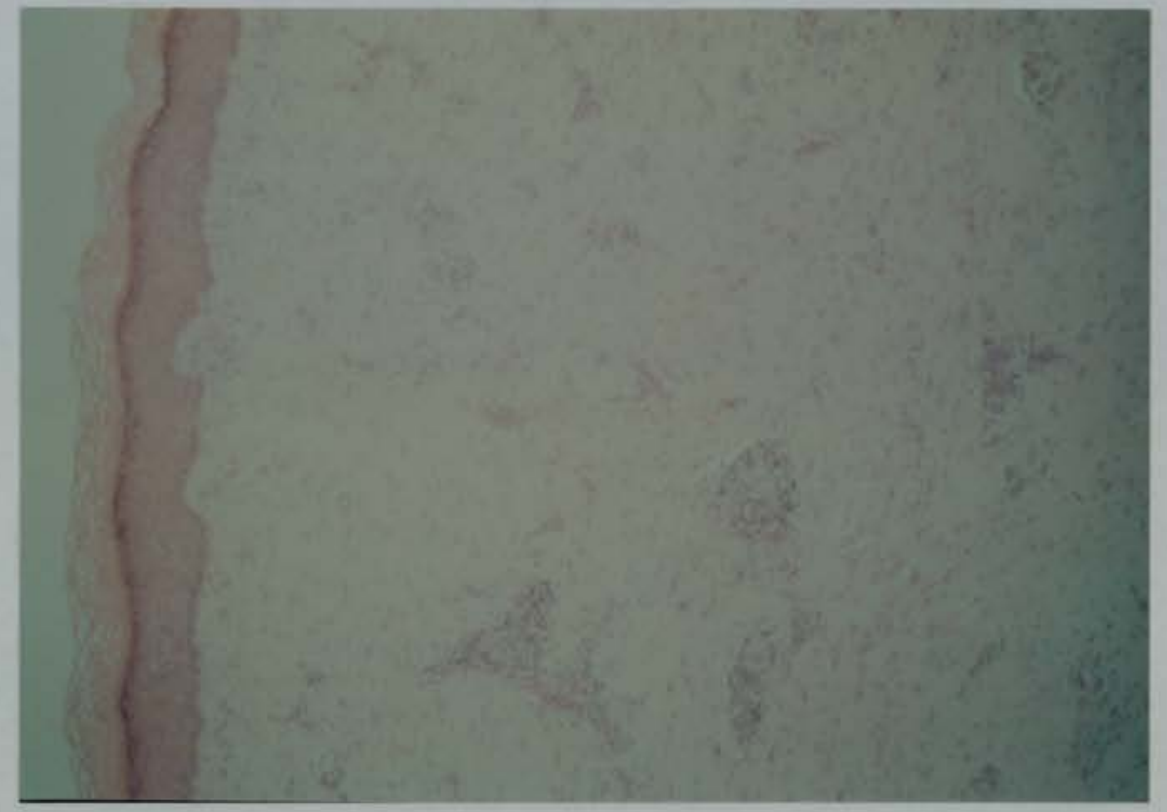

Figure 6.6: Impression smear from a lesion caused by Lmm.

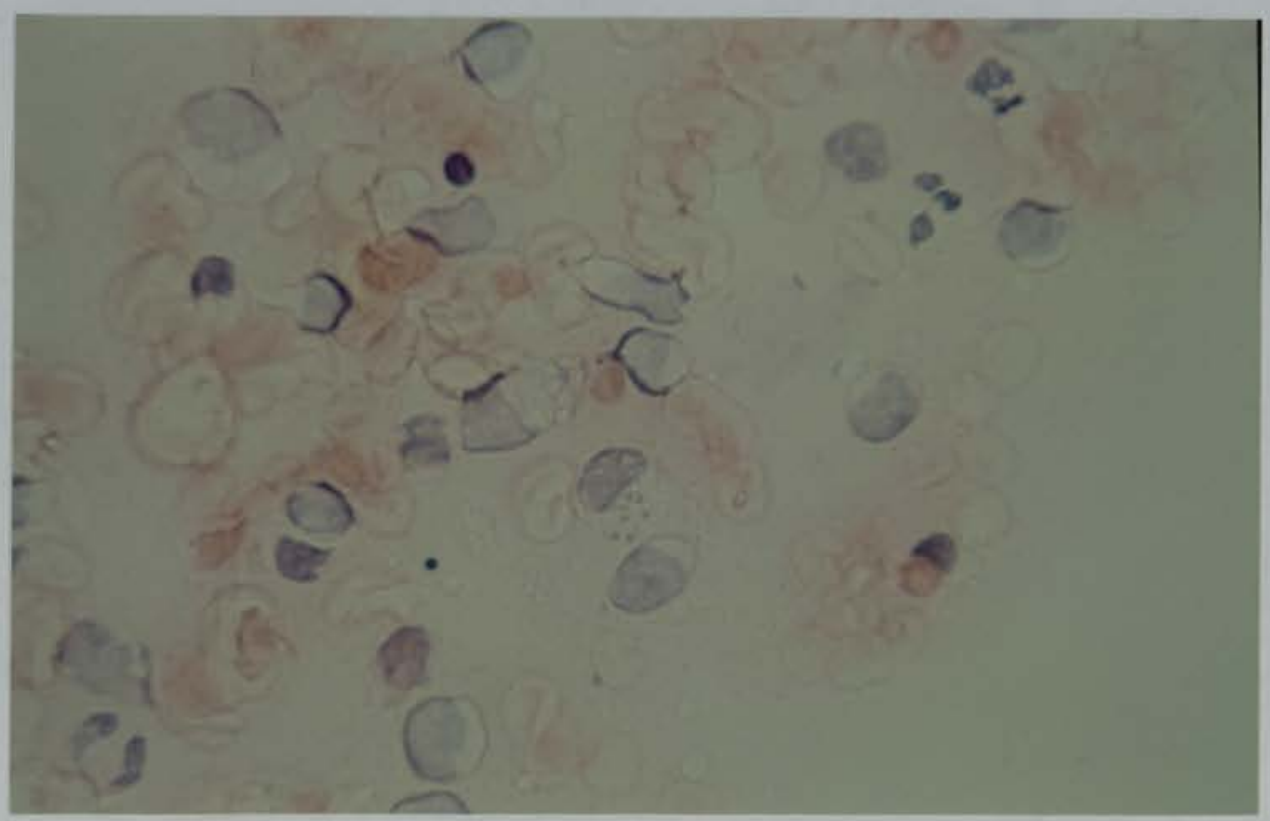




\subsection{Discussion}

The pathological changes seen in $\mathrm{CL}$ show enormous variation from one geographical area to another reflecting differences in the endemic species and, perhaps, the genetic background of the patients. Attempts have been made to develop a histological classification analogous to the spectrum developed for leprosy, but the pathological diversity of CL has made the development of a widely applicable classification very difficult (Ridley 1980, Ridley \& Ridley 1983). Even when a single location has been studied and descriptive histological classifications developed, the interrelationships between the histological patterns is not clear and their prognostic value uncertain. However, some characteristic histological features of New World lesions due to Lbb and Lmm have been identified (Ridley 1979, Ridley 1980, Ridley et al 1980, Ridley \& Ridley 1983, Gutierrez et al 1991). In general, there are small numbers of parasites with an inverse correlation between their detection and the age of the lesion. Destructive changes are seen in both the dermis and epidermis and these are often extensive and more marked than in Old World CL. The pre-treatment biopsies in this study showed all of these features. Parasites were typically scanty with only 7 of 36 biopsies scoring $3+$ or more on the semi-quantitative scoring system used. No correlation was found between the duration of the lesions and the number of parasites seen, but the number of cases was small and the lesions were all of short duration (Ridley et al 1980). Dermal necrosis was usually present and it was extensive in 10 biopsies. Ridley and Ridley (1983) described palisading of macrophages and granulomatous inflammation around areas of dermal necrosis in Belizian CL and this was seen frequently in these cases. Epidermal ulceration and florid hyperplasia were also common.

During histological assessment an attempt was made to classify the pathological appearances using both classifications described by Ridley (Ridley et al 1980, Ridley 1987). These classifications were difficult to use with many individual cases not fitting readily into a single group; this has been the experience of others (Bittencourt \& Barral 1991 ).

Some general principles relating histology to prognosis have been proposed. The presence of epithelioid and giant cells has been associated with a good 
response to treatment and healing (Ridley 1980, Gutierrez et al 1991). The prognostic significance of dermal necrosis is unclear. Some studies suggest that a necrotising reaction either within the granulomas or in the dermal collagen is associated with the rapid elimination of parasites and a good prognosis (Ridley 1980), whilst others found that necrosis was associated with resistance to drug therapy (Gutierrez et al 1991). Nine of the 34 lesions in this study failed to heal following one course of treatment, and, of these, 7 included areas of dermal necrosis and 2 included epithelioid cell granulomas. There were no histological features predictive of non-responsive lesions prior to treatment. However, these patients were participating in a randomised comparative trial of aminosidine and sodium stibogluconate and, as 7 of the 9 lesions which failed to heal were treated with aminosidine this may reflect a difference in drug efficacy rather than inherent differences in the lesions. The results of this trial are reported in chapter 5 .

Healed biopsies were characterised by dermal fibrosis with a scanty chronic inflammatory cell infiltrate together with well formed granulomas in approximately half the biopsies.

Impression smears were of no additional value to the histological sections. It has been suggested that they are useful as a rapid diagnostic technique in CL (Berger et al 1987). However, speed is not critical and, even if it were, rapid processing techniques would allow examination of fixed paraffin-embedded tissue on the same working day. In this series, as previously described in New World CL, parasites were very scanty in many of the histological sections and in none of these cases were parasites identified in the impression smears. In these cases the associated inflammatory response, dermal and epidermal abnormalities (not available in the impression smears), together with the clinical details, give important additonal clues to the diagnosis.

Immunoperoxidase techniques using polyclonal antibodies raised against leishmania have been found useful for demonstrating LD bodies in formalinfixed tissue (Sells and Burton 1981). These preliminary results, with an antiLmm antibody, demonstrated specific staining of the LD bodies with minimal background staining. However the utility of this antibody is limited by the 
absence of cross-reactivity with Lbb. In view of the difficulties in positively identifying leishmania when organisms are scanty further polyclonals should be developed. Most useful would be one which cross-reacts with all types, but a battery of species specific antibodies would also be of great value.

It is important to assess the adequacy of treatment, particularly in cases caused by Lbb which may cause mucocutaneous disease. The results indicate that the changes observed histologically following treatment confirmed the clinical appearances. Basophilic intracellular fragments were frequently seen in biopsies taken 6 weeks after treatment. Although easily identified, well-formed LD bodies were very infrequent and, provided the inflammatory reaction was resolving, these appearances were not incompatible with healing. No lesion in which this material was seen reactivated provided healing was clinically complete and the culture negative. It is probable that these intracellular fragments represent cellular debris or non-viable leishmania rather than viable parasites; immunocytochemistry may be of use in resolving this problem. Histological examination of a posttreatment biopsy is therefore of limited clinical value.

In contrast, culture, which by definition detects viable organisms, is of value following apparently successful treatment. In one case Lmm was identified in a completely healed scar and in 2 others Lbb was identified in lesions which had re-epithelialised but remained indurated. Those in which Lbb was identified were retreated. Whereas re-epithelialisation is an objective endpoint, which can be easily judged by all physicians, induration is much more subjective; in one case it was minimal and could have been easily overlooked. 
6.6 Summary: A study of the diagnostic pathology.

1. Histological examination and culture of a biopsy from the lesion are complementary techniques which provide a parasitological diagnosis in most cases.

2. Parasites are typically scanty. There is a characteristic dermal infiltrate, composed of lymphocytes, plasma cells histiocytes and giant cells, which is frequently associated with necrosis. This is associated with florid epithelial hyperplasia.

3. The classifications proposed by Ridley were difficult to use.

4. No particular pathological change correlated with prognosis.

5. Giemsa-stained sections provide no advantage over standard H\&E stained sections.

6. Impression smears contribute little to confirming the diagnosis.

7. Immunocytochemistry with specific anti-leishmanial antibodies holds promise for definitive parasite identification. It is likely to be particularly useful in cases where parasites are scanty or if intra-cytoplasmic material of uncertain origin is seen.

8. Following treatment, culture is of more value than histology in assessing the response. 


\section{Chapter 7}

\section{Conclusions and Management Guidelines}

What have these studies told us about Belizian cutaneous leishmaniasis and its management? The retrospective case-note review described in chapter 2 showed that most soldiers had a single lesion consisting of an ulcer on the exposed extremities. The lesions referred for treatment were more often due to Lbb than Lmm. Ulcers caused by Lbb develop more rapidly and are, on average, larger than those caused by $\mathrm{Lmm}$. However, it is not possible to distinguish them by their clinical appearance.

Sodium stibogluconate is an effective treatment. The efficacy of a single course can be increased from $48.5 \%$ to $63.9 \%$ by increasing the total dose administered from $6 \mathrm{~g}(600 \mathrm{mg} /$ day for 10 days $)$ to $16.8 \mathrm{~g}(1200 \mathrm{mg} /$ day for 14 days) (chapter 2). Extrapolation of these results indicated that $24 \mathrm{~g}$ would cure $90 \%$; the study in chapter 5 showed that $20 \mathrm{mg} / \mathrm{kg} / \mathrm{day}$ for 20 days (average total dose $29.2 \mathrm{~g}$ ) cured $88 \%$ but with considerable morbidity during the final 7 days of treatment. This regimen was associated with some toxicity characterised by falls in the white cell and platelets counts, hepatocellular damage and impairment of hepatic metabolic capacity (chapters 3 and 5). There is a progressive fall in ECG T-wave amplitude during treatment, this was reversible. Cardiac function, assessed by echocardiography, is not impaired during treatment and there is no evidence of electrical instability (chapter 4). All patients develop myalgia during treatment and in some there are marked elevations in creatine kinase which persist for months after treatment. This should be investigated further to confirm that it is skeletal, and not cardiac, muscle that is involved and to delineate the nature of inflammation and its relationship to exercise.

In contrast, aminosidine was well tolerated and safe, but it was less efficacious than sodium stibogluconate, particularly against Lbb. It is therefore unlikely to be of value as a treatment for Belizian cutaneous leishmaniasis (chapter 5). 
It can be difficult to confirm a clinical diagnosis of American cutaneous leishmaniasis. In the retrospective study this was only achieved in $61 \%$ of cases. However, culture and histological examination of a biopsy from the edge of a lesion permits a diagnosis in nearly all cases. There are fewer parasites present in lesions due to Lbb, but the characteristic inflammatory infiltrate can alert the pathologist to their presence. Preliminary results indicate that immunohistochemistry may facilitate the positive identification of scanty parasites in otherwise equivocal sections. A polyclonal which reacts with all types of leishmania should now be developed. The response to treatment can be judged clinically 6 weeks after the end of treatment. This should be supplemented by an attempt to culture the parasite from the lesion. Viable organisms may be persist in the presence of only minimal induration which can be difficult to evaluate (chapter 6). Relapses can occur for up to 2 years (chapter 2).

On the basis of these results I propose some management guidelines (figure 7.1). The diagnosis should be confirmed histologically and by culture from a biopsy taken from the active edge of the lesion.. Sodium stibogluconate $20 \mathrm{mg} / \mathrm{kg} /$ day iv should be administered for 20 days. The result of treatment should be assessed 6 weeks after the completion of treatment both clinically complete re-epithelialisation with a flat, non-indurated, scar, and by culture of a biopsy. Treatment may be repeated if the lesion has not healed or if the post-treatment culture is positive. If a second course of sodium stibogluconate is ineffective then alternatives, such as ketoconazole or pentamidine, could be considered.

Treatment should be carefully monitored. A full blood count, urea and creatinine, liver function tests, creatine kinase and lactate dehydrogenase should be measured and an ECG performed before treatment, weekly during treatment and 6 weeks after treatment. Monitoring should contiue thereafter until the parameters return to normal. Patients should be kept under review for 2 years to detect relapses. 


\section{Figure 7.1}

\section{Management Guidelines}

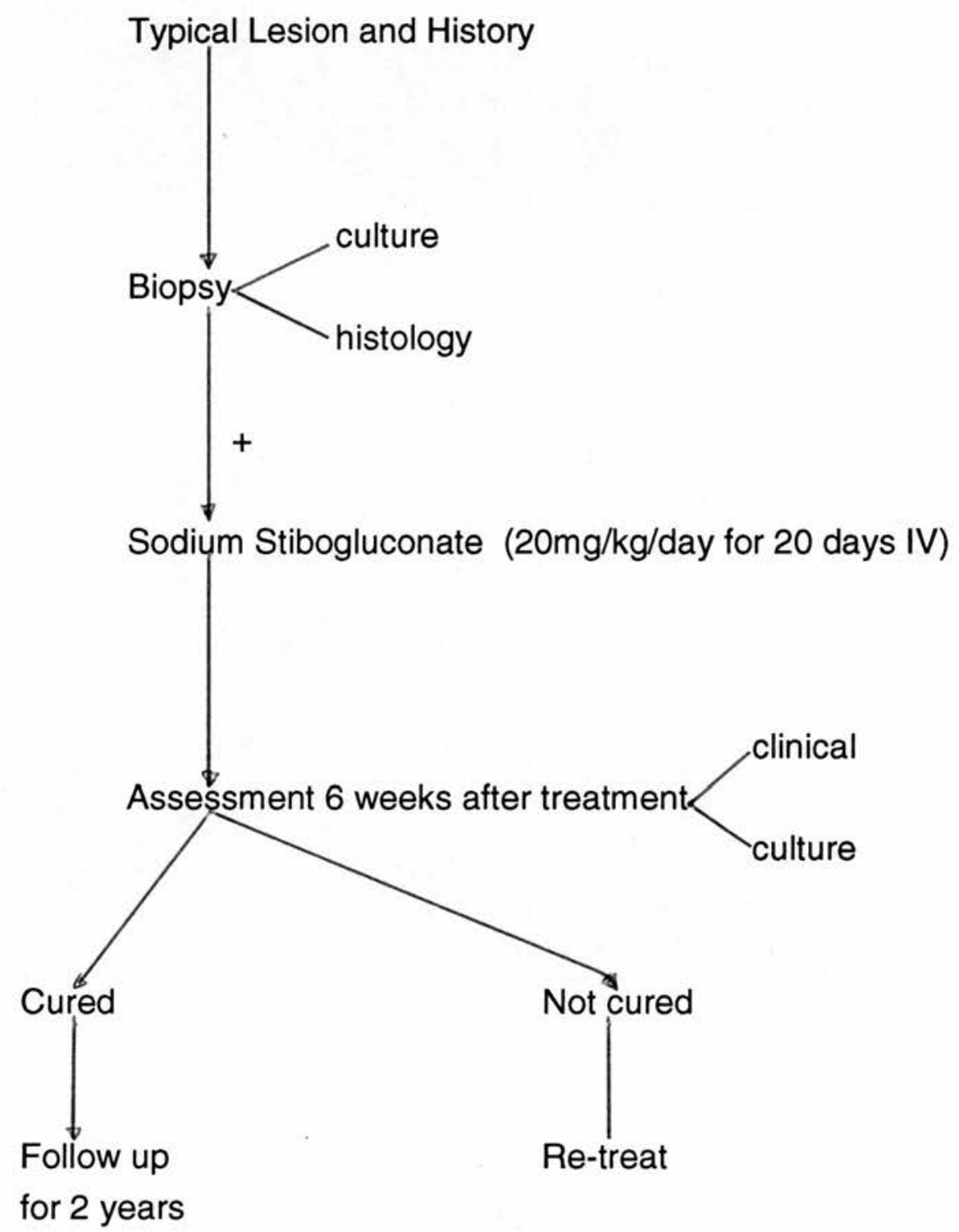

\begin{tabular}{|c|c|c|c|}
\hline \multirow[t]{4}{*}{ Monitoring: } & FBC & 1 & Pre - treatment \\
\hline & U/Es, LFTs & I & weekly during treatment \\
\hline & CK, LDH & I & 6 weeks after treatment \\
\hline & ECG & I & \\
\hline
\end{tabular}




\section{References}

Adachi Y, Horii K, Takahashi Y, Tanihata M, Ohba Y, Yamamoto T (1980): Serum glutathione S-transferase activity in liver diseases. Clinica Chimica Acta 106; 243-255.

Aebischer T (1994): Recurrent cutaneous leishmaniasis: a role for persistent parasites? Parasitology Today 10; 25-28.

Akovbyan AA, Mukhamedov SM (1968): Treatment of cutaneous leishmaniasis of acutely necrotising type (Borovsky disease) with monomycin. (abstract) Tropical Diseases Bulletin 65; 987.

Al-Gindan Y, Kubba R, El-Hassan AM et al (1989): Dissemination in cutaneous leishmaniasis Lymph node involvement. International Journal of Dermatology 28; 248-254.

Alder S, Ber M (1941): The transmission of leishmania tropica by the bite of Phlebotomus papatasii Indian Journal of Medical Research 29; 803809.

Alder S, Theodor O (1925): cited in Chulay JD (1991).

Anonymous (1961): Antimony and the Heart. British Medical Journal 1; 16651666.

Anonymous (1989): Pentostam data sheet. In: ABPI Data Sheet Compendium 1989-1990. London, Datapharm.

Antezana G, Zaballos R, Mendoza C, Lyevre P, Cardenas F, Noriega I, Ugarte H, Dedet JP (1992): Electrocardiographic alterations during treatment of mucocutaneous leishmaniasis with meglumine antimoniate and allopurinol. Transactions of the Royal Society of Tropical Medicine and Hygiene 86; 31-33.

Anthony RL, Grogl M, Sacci JB, Ballou RW (1987): Rapid detection of leishmania amastigotes in fluid aspirates and biopsies of human tissue. American Journal of Tropical Medicine and Hygiene 37; 271-276.

Arcamone F, Bertazzoli C, Chione M, Scotti T (1959): Aminosidina: un nuovo antibiotico oligosaccaridico. Giornale de la Microbiologica 7; 251-272.

Ashford RW, Desjeux P, de Raadt P (1992): estimation of population at risk of infection and number of cases of leishmaniasis. Parasitology Today 8; 104-105.

Ballou WR, McLain JB, Gordon DM, Shanks GD, Andujar J, Berman JD, Chulay JD (1987): Safety and efficacy of high dose sodium 
stibogluconate therapy of American cutaneous leishmaniasis Lancet ii; 13-16.

Barker DC, Bunker J, Gibson LJ, Williams RH (1986): Characterisation of leishmania Sp. by DNA hybridization probes - a laboratory manual. Geneva, UNDP/World Bank/WHO (TDR).

Barrios LA, Costa JML, Netto EM, Vexenat COR, Cubba CC, Marsden PD (1986): Intralesional glucantime in leishmania braziliensis braziliensis infections. Transactions of the Royal Society of Tropical Medicine and Hygiene (letter) 80; 173-174.

Bass NM, Kirsch RE, Tuff SA, Saunders Sj (1978): Radioimmmunoassay of plasma ligandin. A sensitive index of experimental hepatocellular necrosis. Gastroenterology 75; 589-594.

Beckett GJ, Chapman BJ, Dyson EH, Hayes JD (1985a): Plasma glutathione S-transferase measurements after paracetamol overdosage: evidence for early hepatocellular damage Gut 26; 26-31.

Beckett GJ, Dyson EH, Templeton AJ, Hayes JD (1985b): Plasm a glutathione $\mathrm{S}$-transferase measurements by radioimmunoassay. $\mathrm{A}$ sensitive index of hepatocellular damage in man. Clinica Chimica Acta 146; 11-19.

Beckett GJ, Hayes JD (1987): Plasma glutathione S-transferase measurements and liver disease in man. Journal of Clinical Biochemistry and Nutrition 2; 1-24.

Beckett GJ, Hayes JD (1993): Glutathione S-transferases: biomedical applications. In: Spiegel HE (ed) Advances in Clinical Chemistry 30; 281-380.

Benchimol A, Matsuo S (1971): Ejection time before and after aortic valve replacement. American Journal of Cardiology 27; 244-249.

Berger RS, Perez-Figaredo RA, Spielvogel RL (1987): Leishmaniasis: The touch preparation as a rapid means of diagnosis. Journal of the American Academy of Dermatology 16; 1096-1105.

Berger T, Meltzer MS, Oster CN (1985): Lymph node involvement in leishmaniasis. Journal of the American Academy of Dermatology 12; 993-996.

Beckett GJ, Kellet HA, Gow SM, Hussey AJ, Hayes JD, Toft AD (1985): Elevated plasma glutathione S-transferase concentrations in hypothyroid patients receiving thyroxine replacement: Evidence for hepatic damage. British Medical Journal 291; 427-431. 
Berman JD (1988): Chemotherapy for leishmaniasis: Biochemical mechanisms, clinical efficacy, and future strategies. Reviews of Infectious Diseases 10; 560-586.

Berman JD (1991): Biochemical mechanisms of clinical antileishmanial agents: a review. Journal of Cellular Pharmacology 2; 75-82.

Berman JD, Grogl M (1988): Leishmania mexicana: chemistry and biochemistry of sodium stibogluconate (Pentostam). Experimental Parasitology 67; 96-103.

Bittencourt AL, Barral A (1991): Evaluation of the histopathological classifications of American cutaneous and mucocutaneous leishmaniasis. Memorias Do Instituto Oswaldo Cruz 86; 51-56.

Bogdan C, Stosiek N, Fuchs H, Röllinghoff M, Solbach W (1990): Detection of potentially diagnostic leishmanial antigens by Western blot analysis of sera from patients with Kala-azar or multi-lesional cutaneous leishmaniasis. Journal of Infectious Diseases 162; 1417-1418.

Bonow RO, Bacharach SL, Green MU, Kent KM, Rosing DR, Lipson LC, Leon MB, Epstein SE (1981a): Impaired left ventricular diastolic filling in patients with coronary artery disease: assessment with radionuclide angiography. Circulation $64 ; 315-323$.

Bonow RO, Rosing DR, Bacharach SL, Green MV, Kent KM, Lipson LC, Maron BJ, Leon MB, Epstein SE (1981b). Effects of verapamil on left ventricular systolic function and diastolic filling in patients with hypertrophic cardiomyopathy. Circulation 64; 787-796.

Bromberger-Barnea B, Stephens NL (1965): Effects of antimony on myocardial performance in isolated and intact canine hearts. American Industrial Hygiene Association Journal 26; 404-408.

Brooks J (1983): The 1984 South America Handbook. Bath, Trade and Travel Publications. p1166-1180.

Bryceson ADM (1992): Leishmaniasis. In: Champion RH, Burton JL, Ebling FJG Textbook of Dermatology, 5th Ed. London, Blackwell Scientific. p1251-1263.

Bryceson ADM (1987): Therapy in man. In: Peters W, Killick-Kendrick R (eds). The leishmaniases in biology and medicine, volume 2. London, Academic Press. p847-907. 
Burgess NRH (1981): Arthropods of medical importance. Chilbolton, Noble. p67-72.

Burkle WS (1981): Comparative evaluation of the aminosidine antibiotics for systemic use. Drug Intelligence and Clinical Pharmacy 15; 847-861.

Castro C, Sampaio RN, Marsden PD (1990): Severe arthralgia, not related to dose, associated with pentavalent antimonial therapy for mucosal leishmaniasis. Transactions of the Royal Soceity of Tropical Medicine and Hygiene 84; 362.

Chulay JD (1991): Leishmaniasis. In: Strickland GT (ed) Hunter's Tropical Medicine, 7 Ed. Philadelphia, Saunders. p638-655.

Chulay JD, Bhatt SM, Muigai R, Ho M, Gachihi G, Were JBO, Chunge C, Bryceson ADM (1983): A comparason of three dosage regimens of sodium stibogluconate in the treatment of visceral leishmanisis in Kenya. Journal of Infectious Diseases 48; 148-155.

Chulay JD, Spencer HC, Mugambi M (1985): Electrocardiographic changes during treatment of leishmaniasis with pentavalent antimony (sodium stibogluconate). American Journal of Tropical Medicine and Hygiene 34; 702-709.

Chunge CN, Owate J, Pamba HO, Donno L (1990): Treatment of visceral leishmaniasis in Kenya by aminosidine alone or combined with sodium stibogluconate. Transactions of the Royal Society of Tropical Medicine and Hygiene: $84 ; 221-225$.

Clarkson P, Wheeldon NM, MacDonald TM (1994): Left ventricular diastolic dysfunction. Quarterly Journal of Medicine 87; 143-148.

Cole ACE (1944): Kala-azar in East Africa. Transactions of the Royal Society of Tropical Medicine and Hygiene 37; 409-435.

Copeland HW, Arana BA, Navin TR (1990): Comparason of active and passive case detection of cutaneous leishmaniasis in Guatemala. American Journal of Tropical Medicine and Hygiene 43; 257-259.

Cunningham DD (1885): On the presence of peculiar parasitic organisms in the tissue culture of a specimen of Dehli boil. Scientific Memoirs of Medical Officers of the Army of India 1; 21-31.

Davis A (1961): The effects of antimony dimercaptosuccinate on the electrocardiogram. British Heart Journal 23; 291-296.

de Bruijn MHL, Barker DC (1992): Diagnosis of New World leishmaniasis: specific detection of species of the leishmania braziliensis complex by amplification of kinetoplast DNA. Acta Tropica 52; 45-58. 
de Rossell RA, de Duran RdeJ, Rossell O, Rodríguez AM (1992) Is leishmaniasis ever cured? Transactions of the Royal Society of Tropical Medicine and Hygiene 86; 251-253.

Dempsey J (1965): Leishmaniasis in the Sudan Republic (26).

Electrocardiographic findings in Sudanese Kala-azar. East African Medical Journal 42; 131-134.

Dollery C (ed) (1991): Therapeutic drugs, volume 2. London, ChurchillLivingston. pS56-S59.

Donno L, Rimoldi R (1974): Trattamento con aminosidina per perfusione venosa nelle broncopneumopatie. Gazzetta Medica Italiana 331; 377383.

Donovan C (1903): On the possibility of the occurrence of trypanosomiasis in India. British Medical Journal ii; 79.

EL-On J, Weinrauch L, Livishin R, Even-Paz Z, Jacobs GP (1985): Topical treatment of recurrent cutaneous leishmaniasis with ointment containing paromomycin and methylbenzethonium chloride. British Medical Journal 291; 704-705.

El-On J, Cawich F, Evans DA, Weinrauch L (1993): Topical treatment of cutaneous leishmaniasis in Belize: in vitro and in vivo studies with Leishmania mexicana. International Journal for Parasitology 23; 121127.

El-On J, Halevy S, Grunwald MH, Weinrauch L (1992): Topical treatment with Old World cutaneous leishmanisis caused by Leishmania major: a double blind control study. Journal of the American Academy of Dermatology 27; 227-231.

El-On J, Jacobs GP, Weinrauch L (1988): Topical treatment of cutaneous leishmaniasis. Parasitology Today 4; 76-81.

El-On J, Livishin R, Even-Paz, Hamburger D, Weinrauch L (1986): Topical treatment of cutaneous leishmaniasis. Journal of Investigative Dermatology $87 ; 284-288$.

El-Safi SH, Murphy AG, Bryceson ADM, Neal RA (1990): A double blind clinical trial of the treatment of cutaneous leishmanisis with paromomycin ointment. Transactions of the Royal Society of Tropical Medicine and Hygiene 84; 690-691.

Elgood C (1934): The early history of Bagdad boil. Journal of the Royal Asiatic Society. p519-533.

Ereshov ME (1978): cited in Bryceson (1987). 
Evans D (1989): Handbook on the isolation, characterisation and cryopreservation of Leishmania. Geneva, UNDP/World Bank/WHO.

Evans D, Lanham SM, Balwin CL, Peters W (1984): The isolation and isoenzyme characterization of Leishmania braziliensis subsp. from patients with cutaneous leishmaniasis acquired in Belize. Transactions of the Royal Society of Tropical Medicine and Hygiene 78; 35-42.

Even-Paz Z, Sagher F (1961): Some basic medical problems illustrated with cutaneous leishmaniasis. South African Medical Journal 35; 567-581.

Ewing (1992): Analysis of heart rate variability. In: Bannister R, Mathias CJ (Eds) Autonomic failure, A textbook of clinical disorders of the autonomic nervous system. Oxford, Oxford university Press. p312333.

Ewing DJ, Neilson JMM, Travis P (1984): New method for assessing cardiac parasympathetic activity using 24 hour electrocardiograms. British Heart Journal 53; 396-402.

Firth RH (1891): Notes on the appearance of certain sporozoöid bodies in the protoplasm of an 'oriental sore.' British Medical Journal. i; 252-254.

Fisch C (1992): Electrocardiography and vectorcardiography. In: Braunwald E. Heart disease. A textbook of cardiovascular medicine.

Philadelphia, Saunders p116-160.

Franco MA (1992): Determination of the antimonials $\left(\mathrm{Sb}^{3}\right.$ and $\left.\mathrm{Sb}^{5}\right)$ in drugs. Masters thesis in chemistry. Universidade de Brazília. (Marsden, personal communication).

Freigenbaum H (1986): Echocardiography (4th ed). Philadelphia; Lea and Frebiger.

Fursden E (1988): Belize. Army Quarterly 118; 33-41.

Gade DW (1977): Inca and colonial settlement, coca cultivation and endemic disease in the tropical forest. Journal of Historical Geography 5; 263279.

Gardiner PJ (1977): Taxonomy of the genus Leishmania: a review of nomenclature and classification. Tropical Diseases Bulletin 74; 10641088.

Garnham PCC, Lewis DJ (1959): Parasites of British Honduras with special reference to leishmaniasis. Transactions of the Royal Society of Tropical Medicine and Hygiene 53; $12-35$. 
Goetz PW (ed)( 1991): The countries of Central America: Belize. In: New Encyclopaedia Britannica, 15th ed. London, Encyclopaedia Britannica Inc. p683-686.

Gran GE, Modlin RL (1991): Immune mechanisms in bacterial and parasitic diseases: protective immunity versus pathology. Current Opinion in Immunology 3; 480-485.

Griffiths WAD (1987): Old World Leishmaniasis. In: Peters W, KillickKendrick R (eds) The leishmaniases in biology and medicine, volume 2. London, Academic Press. p617-636.

Grogl M, Oduola AMJ, Cordero LDC, Kyle DE (1989): Leishmania Spp: development of pentostam-resistant clones in vitro by discontinuous drug exposure. Experimental Parasitology 69; 78-90.

Grogl M, Thomason TN, Franke ED (1992): Drug resistance in leishmaniasis: its implication in systemic chemotherapy of cutaneous and mucocutaneous disease. American Journal of Tropical Medicine and Hygiene 47; 117-126

Guderian RH, Chico ME, Rodgers MD, Pattishall KM, Grogl M, Berman JD (1991): Placebo controlled treatment of Ecuadorian cutaneous leishmaniasis. American Journal of Tropical Medicine and Hygiene 45; 92-97.

Guimarães MCS, Celeste BJ, Franco EL (1990): Diagnostic performance indices for immunofluorescent tests and enzyme-linked immunoassays of leishmaniasis sera from Northern and South-Eastern Brazil. Bulletin of the World Health Organisation 68; 39-43.

Gutgesell HP, Paquet M, Duff DF, McNamara DG (1977): Evaluation of left ventricular size and function by echocardiography. Circulation 56; 457-462.

Gutierrez Y, Salinas GH, Palma G, Valderrama LB, Santrich CV, Saravia NG (1991): Correlation between histopathology, immune response, clinical presentation, and evolution in Leishmania braziliensis infection. American Journal of Tropical Medicine and Hygiene 45; 281-289.

Hanham CA, Zhao F, Shaw JJ, Lainson R (1991): Monoclonal antibodies for the identification of New World leishmania. Transactions of the Royal Society of Tropical Medicine and Hygiene $85 ; 220$.

Harms G, Chehade AK, Douba M, Roepke M, Mouakeh A, Rosenkaimer F, Bienzle U (1991): A randomised trial comparing a pentavalent antimonial drug and recombinant interferon gamma in the local treatment of cutaneous leishmaniasis. Transactions of the Royal Society of Tropical Medicine and Hygiene $85 ; 214-216$. 
Harms G, Zwinenberger K, Chéhade AK et al (1989): Effects of intradermal gamma-interferon in cutaneous leishmaniasis. Lancet $\mathbf{i} ;$ 1287-1292.

Hayes JD, Gilligan D, Chapman BJ, Beckett GJ (1983): Purification of human hepatic glutathione S-transferase and the development of a radioimmunoassay for its measurement in plasma. Clinica Chimica Acta 134; 107-121.

Hayes PC, Bouchier IAD (1989): Liver function tests in clinical practice: their uses and limitations. Clinical Chemistry and Enzyme Communications 2; 23-34.

Hayes PC, Hayes JD, Hussey AJ, Bouchier IAD, Beckett GJ (1990): Changes in plasma glutathione S-transferase $\mathrm{B}_{1}$ concentration after alcohol ingestion in man: a measure of hepatocellular sensitivity to chronic alcohol excess. Clinical Chemistry and Enzyme Communications 2; 189-194.

Henderson A, Jolliffe DS (1985): Cardiac effects of sodium stibogluconate. British Journal of Clinical Pharmacology 19; 73-77.

Hepburn NC (1992): Cutaneous Leishmaniasis. Part 1: Clinical Features. Proceedings of the Royal College of Physicians of Edinburgh 22; 448459.

Hepburn NC, Tidman MJ, Hunter JAA (1993): Cutaneous leishmaniasis in British troops from Belize. British Journal of Dermatology 128; 63-68.

Herrer A, Christensen HA (1975): Implication of Phlebotomus sandflies as vectors of bartonellosis and leishmaniasis as early as 1764 . Science 190; 154-155.

Herwaldt BL, Arana BA, Navin TR (1992): The natural history of cutaneous leishmaniasis in Guatemala. Journal of Infectious Diseases 165; 518527.

Herwaldt BL, Berman JD (1992): Recommendations for treating leishmaniasis with sodium stibogluconate (Pentostam) and review of pertinent clinical studies. American Journal of Tropical Medicine and Hygiene 46; 296-306.

Herwaldt BL, Stokes SL, Juranek DD (1993): American cutaneous Leishmaniasis in U.S. Travelers. Annals of Internal Medicine 118; 779 784.

Hiley C, Bell J, Hume R, Strange RC (1989): Differential expression of alpha and pi isoenzymes of glutathione S-transferase in developing human kidney. Biochimica Et Biophysica Acta 990; 321-324. 
Hirchfeld S, Meyer R, Schwartz DC (1975): Measurement of right and left ventricular systolic time intervals by echocardiography. Circulation 51; 304-309.

Honey M (1960): The effects of sodium antimony tartrate on myocardium. British Heart Journal 22; 601-616.

Howie AF, Patrick AW, Fisher BM, Frier BM, Beckett GJ (1989): Plasma hepatic glutathione S-transferase concentrations after insulin-induced hypoglycaemia in normal and diabetic patients. Diabetic Medicine 6; 224-227.

Hussey AJ, Howie J, Allan LG, Drummond G, Hayes JD, Beckett GJ (1986): Impaired hepatocellular integrity during general anaesthesia as assessed by glutathione S-transferase. Clinica Chimica Acta 161; 1928.

Ito R, Kamamuja H, Nakagawa W, Chang HS, Kabayashi S, Ohmoto M, Matsura S, Toida S, Momazawa K (1968): Studies on acute, subchronic and chronic toxicity of aminosidine (AMD) in rats and rabbits. Chemotherapy 16; 124-133.

James DM, Gilles HM (1985): Human anti-parasitic drugs: pharmacology and usage. Chichester, John Willey. p92-104.

Janovy J (1987): Physiology and biochemistry. In: Peters W, Killick-Kendrick $\mathrm{R}$ (eds). The leishmaniases in biology and medicine, volume 1. London, Academic Press. p177-210.

Jones TC, Johnson WD, Barretto AC, Lago E, Badaro B, Cerf B, Reed SG, Netto EM, Tada MS, Franca F, Weise K, Golightly L, Fikrig E, Costa JML, Cuba CC, Marsden PD (1987): Epidemiology of American cutaneous leishmaniasis due to Leishmania braziliensis braziliensis. Journal of Infectious Diseases 156; 73-83.

Kahlmetir G (1979): Gentamicin and tobramycin: clinical pharmacokinetics and nephrotoxicity aspects and assay techniques. Scandanavian Journal of Infectious Disease 18; 1-40.

Kal ow W, Tang BK (1993): The use of caffeine for enzyme assays: a critical appraisal. Clinical Pharmacology and Therapeutics 53; 503-514.

Kalter DC (1989): Cutaneous and mucocutaneous leishmaniasis. Progress in Dermatology 23; 1-11.

Kaplan RJ, Wilkin JL, Hartman DL (1978): Treatment of Leishmaniasis with Sodium antimony gluconate: transitory ECG changes. Southern Medical Journal 71; 469-470. 
Kellum RE (1986): Treatment of cutaneous leishmaniasis with an intralesional antimonial drug (Pentostam). Journal of the American Academy of Dermatology 15; 620-622.

Kleiger RE, Miller JP, Bigger JT, Moss AJ (1987): Decreased heart rate variability and its association with increased mortality after acute myocardial infarction. American Journal of Cardiology 59; 256-262.

Kreutzer RD, Christensen HA (1980): Characterization of Leishmania Spp by isoenzyme electrophoresis. American Journal of Tropical Medicine and Hygiene 29; 199-208.

Kreutzer RD, Souraty N, Semko ME (1987): Biochemical differences among Leishmania species and subspecies. American Journal of Tropical Medicine and Hygiene 36; 22-32.

Kubba R, Al-Gindan Y (1989): Leishmaniasis. Dermatology Clinics 7; 331351.

Kubba R, Al-Gindan Y, El-Hassan AM, Omer AHS (1987a): Clinical diagnosis of cutaneous leishmaniais (oriental sore). Journal of the American Academy of Dermatology 16; 1183-1189.

Kubba R, El-Hassan AM, Al-Gindan Y et al (1987b): Dissemination in cutaneous leishmaniasis 1. Subcutaneous nodules. International Journal of Dermatology 26; 300-304.

Labovitz AJ, Pearson AC (1987): Evaluation of left ventricular diastolic function: Clinical relevance and recent Doppler echocardiographic insights. American Heart Journal 114; 836-861.

Lainson R, Shaw JJ (1987): Evolution, classification and goegraphical distribution. In: Peters W, Killick-Kendrick R (eds). The leishmaniases in biology and medicine, volume 1. London, Academic Press. p1-120.

Lainson R, Strangeways-Dixon J (1963): Leishmania mexicana: the epidemiology of dermal leishmaniasis in British Honduras.

Transactions of the Royal Society of Tropical Medicine and Hygiene 57; 242-265.

Leishman WB (1903): On the possibility of the occurrence of trypanosomiasis in India. British Medical Journal i; 252-254.

Lerner EA (1993): Vector biology in leishmaniasis transmission: new findings. In: Burgdorf WHC, Katz Sl (eds). Dermatology progress and prespectives: Proceedings of the 18th World Congress of Dermatology. New York, Parthenon. p396-398.

Lever WF, Schaumberg-Lever G (1990) Histopathology of the Skin (7ed). Philadelphia, Lippincott. p394-398. 
Lewis DJ, Ward RD (1987): Transmission and vectors. In: Peters W, KillickKendrick R (Eds) The leishmaniases in biology and medicine, volume 1. London, Academic Press. p235-262.

Lewis RP, Leighton RF, Forester WF, Weissler AM (1974): In: Weissler AM (Ed). Non-Invasive Cardiology. New York, Grune and Stratton. p301368.

Liew FY (1989): Immunity to protozoa. Current Opinion in Immunology 1; 441-447.

Llanos-Cuentas EA, Cuba CC, Barreto AC, Marsden PD (1984): Clinical characteristics of human Leishmania braziliensis braziliensis infections. Transactions of the Royal Society of Tropical Medicine and Hygiene 78; 845-846.

Locksley RM, Louis JA (1992): Immunology of leishmaniasis. Current Opinion in Immunology 4; 413-418.

Lopez M, Montoya Y, Arana M, Cruzalegni F, Braga J, Llanos-Cuentas A, Romero G, Arevalo J (1988): The use of non-radioactive DNA probes for the characterisation of leishmania isolates from Peru. American Journal of Tropical Medicine and Hygiene 38, 308-314.

Lucas S, Kirkham N (1991) Infective Granulomas. In: Kirkham N (ed). Biopsy Pathology of the Skin. London, Chapman and Hall. p311-315.

Magill AJ, Grögl M, Gasser RA, Sun W, Oster CN (1993): Visceral leishmaniasis caused by Leishmania tropica in veterans of operation Desert Storm. New England Journal of Medicine 328; 1383-1387.

Manson-Bahr PEC (1987): Diagnosis. In: Peters W, Killick-Kendrick R (eds). The leishmaniases in biology and medicine, volume 2 . London, Academic Press. p703-729.

Marsden PD, Sampaio RNR, Carvalho EM, Veiga JPR, Costa JLM, LlanosCuentas EA (1985): High continuous antimony therapy in two patients with unresponsive mucosal leishmaniasis. American Journal of Tropical Medicine and Hygiene 34; 710-713.

Marsden PD. (1985): Pentavalent antimonials: old drugs for new diseases. Revista da Sociedade Brasileira de Medicina Tropical 18; 187-198.

Mason SJ, Fortuin NJ (1978): The use of echocardiography for quantitative evaluation of left ventricular function. Progress in Cardiovascular Disease 21; 1072-1083. 
Mattock NM, Peters W (1975a): The experimental chemotherapy of leishmaniasis 1: Techniques for the study of drug action in tissue culture. Annals of Tropical Medicine and Parasitology 69; 349-357.

Mattock NM, Peters W (1975b): The experimental chemotherapy of leishmaniasis 2: The activity in tissue culture of some anti-parasitic and anti-microbial compounds in clinical use. Annals of Tropical Medicine and Parasitology 69; 359-371.

Mauel J, Behin R (1987): Immunity: clinical and experimental. In: Peters W, Killick-Kendrick R (eds). The leishmaniases in biology and medicine, volume 2. London, Academic Press. p731-791.

McAreavey D, Neilson JMM, Ewing DJ, Russel DC (1989): Cardiac parasympathetic activity during the early hours of acute myocardial infarction. British Heart Journal 62; 165-170.

McIntyre N, Rosalki S (1991): Biochemical investigations in the management of liver disease. In: McIntyre N, Benhamou JP, Bircher J, Rizzetto M, Rodes J (eds) Oxford Textbook of Clinical Hepatology, Volume 1. Oxford, Oxford University Press. p293-314.

McKee PH (1989): Pathology of the skin. London, Gower. p4.50-4.52.

McMahon-Pratt D, David JR (1981): Monoclonal antibodies that distinguish between New World species of leishmania. Nature 291; 581-582.

Merx W, Yoon MS, Han J (1977): The role of local disparity in conduction and recovery time on ventricular vulnerability to fibrillation. American Heart Journal 94: 603-610.

Miller LH, Scott P (1989): Immunity to protozoa. Current Opinion in Immunology 2; 368-374.

Moll H (1993): Epidermal Langerhans cells are crucial for immunoregulation of cutaneous leishmaniasis. Immunology Today 14; 383-387.

Mukềrjee A, Seth M, Bhaduri AP (1990): Present status of leishmaniasis. Progress in Drug Research 34; 448-490.

Multicentre Post Infarction Research Group (1983): Risk stratification and survival after myocardial infarction. New England Journal of Medicine 309; 331-336.

Murray A, Ewing DJ, Campbell IW, Neilson JMM, Clarke BF (1975): RR interval variations in young male diabetics. British Heart Journal 37; 882-885.

Navin TR, Arana BA, Arana FE, Berman JD, Chjóu JF (1992): Placebocontrolled clinical trial of sodium stibogluconate (Pentostam) versus 
ketoconazole for treating cutaneous leishmaniasis in Guatemala. Journal of Infectious Diseases 165; 528-534.

Navin TR, Arana FE, de Merida M, Arana BA, Castillo Al, Silvers DN (1990): Cutaneous leishmaniasis in Guatemala: comparason of diagnostic methods. American Journal of Tropical Medicine and Hygiene 42; 3642.

Neal RA (1968): The effect of antibiotics of the neomycin group on experimental cutaneous leishmaniasis. Annals of Tropical Medicine and Parasitology 62; 54-62.

Neal RA (1987): Experimental chemotherapy. In: Peters W, Killick-Kendrick $\mathrm{R}$ (eds). The leishmaniases in biology and medicine, volume 2. London, Academic Press. p792-845.

Neilson JMM(1975): Computer detection of ventricular ectopic beats: On line and Off. In: Computers in cardiology. Los Alamitos, IEEE Computer Press. p33-35.

Nicolle C (1908): Nouvelles acquisitions sue le Kala-azar: cultures; inoculation au chein; étiologie. Comptes Rendus De L'Academie des Sciences 146; 498-499.

Nishimura RA, Abel MD, Hatle LK, Tajik AJ. (1989): Assessment of diastolic function of the heart; Background and current applications of Doppler echocardiography. Part II. Clinical studies. Mayo Clinic Proceedings 64; 181-204.

Nolan J, Flapan AD, Capewell S, MacDonald TM, Neilson JMM, Ewing DJ. (1992): Decreased cardiac parasympathetic activity in chronic heart failure and its relation to left ventricular function. British Heart Journal $67 ; 482-485$.

Olliaro P, Bryceson ADM (1993): Practical progress and new drugs for changing patterns of leishmaniasis. Parasitology Today 9; 296-306.

Oster CN, Chulay JD, Hendricks LD, Pamplin CL, Ballou R, Berman JD, Takafuji ET, Tramont EC, Cranfield CJ (1985): American cutaneous leishmaniasis: a comparason of three sodium stibogluconate schedules. American Journal of Tropical Medicine and Hygiene 34; 856-860.

Pappas MG, McGreevy PB, Hajkowski R, Hendricks L, Oster CN, Hockmeyer WT (1983): Evaluation of promastigote and amastigote antigens in the indirect flourescent antibody test for American cutaneous leishmaniasis. American Journal of Tropical Medicine and Hygiene 32; 1260-1267. 
Pessôa SB (1961): Classificaçâo das leishmanioses e das espécies do genero Leishmania. Arquivos de Hygiene e Saude Publica 26; 41-60.

Peters BS, Fish D, Golden R, Evans DA, Bryceson ADM, Pinching AJ (1990): Visceral leishmaniasis in HIV infection and AIDS: clinical features and response to therapy. Quarterly Journal of Medicine 283; 1101-1111.

Pierini LE (1969): South American Leishmaniasis. In: Simons RDG, Marshall $\mathrm{J}$ (Eds) Essays on Tropical Dermatology. Amsterdam, Exerpta Medical. p175-203.

Presig R (1986): Clinical evaluation of liver functon. In: Thomas HC, Jones AE (eds) Recent Advances in Hepatology. Edinburgh, Churchill Livingston $\mathrm{p} 1-12$.

Rappaport AM (1980): Morphologic aspects and physiological regulation. International Review of Physiology 21; 1-63.

Redick JA, Jakoby WB, Baron J (1982): Immunohistochemical localisation of glutathione S-transferase in livers of untreated rats. Journal of Biological Chemistry 257; 15200-15203.

Renner E, Weitholtz H, Huguenin P, Presig R (1984) Caffeine: a model compound for measuring liver function. Hepatology 4 ; 38-46.

Reynolds JEF (ed) (1993): Martindale: The Extra Pharmacopea 30th ed. London, Pharmaceutical Press. p190-191.

Ribeiro JMC, Vachereau A, Modi GB, Tesh RB (1989): A novel vasodilatory peptide from the salivary glands of the sandfly Lutzomyia longipalpis. Science 243; 212-214.

Ridley DS (1979): The pathogenesis of cutaneous leishmaniasis.

Transactions of the Royal Society of Tropical Medicine and Hygiene 73; $150-160$.

Ridley DS (1980): A histological classification of cutaneous leishmaniasis and its geographical expression. Transactions of the Royal Society of Tropical Medicine and Hygiene 74; 515-521.

Ridley DS (1987): Pathology. In: Peters W, Killick-Kendrick R (eds). The Leishmaniases in Biology and Medicine, vol 2. London, Academic Press. p665-701.

Ridley DS, Marsden PD, Cuba CC, Barreto AC (1980): A histological classification of mucocutaneous leishmaniasis in Brazil and its clinical evaluation. Transactions of the Royal Society of Tropical Medicine and Hygiene 74; 508-514. 
Ridley DS, Ridley MJ (1983): The evolution of the lesion in cutaneous leishmaniasis. Journal of Pathology 141; 83-96.

Rogers $L$ (1904): Preliminary note on the development of trypanosema in cultures of the Cunningham-Leishman-Donovan bodies of cachexial fever and Kala-azar. Lancet ii; 215-216.

Saenz RE, Paz H (1990) Efficacy of Ketoconazole against Leishmania braziliensis panamensis cutaneous leishmaniasis. American Journal of Medicine 89; 147-155.

Sahn DJ, DeMaria A, Kisslo J, Weyman A (1978): Recommendations regarding quantification in M-mode echocardiography: Results of a survey of echocardiographic measurements. Circulation 58; 1072 1083.

Salinas G, Valderrama L, Palma G,Montes G, Saravia NG (1990): Detection of amastigotes in cutaneous and mucocutaneous leishmaniasis using the immunoperoxidase method, using polyclonal antibody: sensitivity and specificity compared with conventional methods of diagnosis. Memorias Do Instituto Oswaldo Cruz 84; 53-60.

Sapire DW, Silverman NH(1970): Myocardial involvement in antimonial therapy; a case report of acute antimony poisoning with serial ECG changes. South African Medical Journal 44; 948-950.

Saravia NG, Weigle K, Segura I, Giannini SH, Pacheco R, Labrada LA, Goncalves A (1990): Recurrent lesions in human Leishmania braziliensis infection - reactivation or reinfection? Lancet 336; 398402.

Schillings RT, Schaffner CP (1961): Differentiation of catenulin-neomycin antibiotics: identity of catenulin, paromomycin, hydroxymycin and aminosidin. Antimicrobial Agents and Chemotherapy 4; 274-285.

Scott HH (1939): A history of tropical medicine. London, Edward Arnold. p548-569.

Scott JAG, Davidson RN, Moody AH, Grant HR, Felmingham D, Scott GMS, Olliaro P, Bryceson ADM (1992): Aminosidine (paromomycin) in the treatment of leishmaniasis imported into the United Kingdom. Transactions of the Royal Society of Tropical Medicine and Hygiene 86; 617-619.

Seaman J, Pryce D, Sondorp HE, Moody A, Bryceson ADM, Davidson RN (1993): Epidemic visceral leishmaniasis in Sudan: a randomised trial of aminosidine plus sodium stibogluconate versus sodium stibogluconate alone. Journal of Infectious Diseases 168; 715-720. 
Sells PG and Burton M (1981): Identification of Leishmania amastigotes and their antigens in formalin fixed tissue by immunoperoxidase staining. Transactions of the Royal Society of Tropical Medicine and Hygiene 75; 461-468.

Sergent E et al (1921): cited in Pierini LE (1969).

Setchell KDR, Welsh MB, Klooster MJ, Balistreri WF (1987): Rapid highperformance liquid chromatography assay for salivary and serum caffeine following an oral load. An indicator of liver function. Journal of Chromatography 385; 267-274.

Sherman M, Bass NM, Campbell JAH, Kirsch RE (1983): Radioimmunoassay of human ligandin. Hepatology 3; 162-169.

Somers K, Rosanelli JD (1962) Electrocardiographic effects of antimony dimercaptosuccinate (Astiban). British Heart Journal 24; 187-191.

Spirito P, Maron BJ (1988): Doppler echocardiography for assessing left ventricular diastolic function. Annals of Internal Medicine 109; 122126.

Stemmer KL (1976): Pharmacology and toxicology of heavy metals: antimony. Pharmacology and Therarapeutics Acta 1; 157-160.

Stockman PK, Beckett GJ, Hayes JD (1985): Identification of a basic hybrid glutathione S-transferase from human liver. Biochemical Journal 227; 457-465.

Surawicz B (1970): Effects of drugs on the electrocardiogram. Progress in Cardiovascular Disease 13; 26-55.

Takafuji ET, Hendricks LD, Danbek JL, McNeil KM, Scagliola HM, Diggs CL (1980): Cutaneous leishmaniasis associated with jungle training. American Journal of Tropical Medicine and Hygiene 29; 516-520.

Thakur CP, Olliaro P, Gothoskar S, Bhowmick S, Choudhury BK, Prasad S, Kumar M, Verma BB (1992): Treatment of visceral leishmaniasis (kala-azar) with aminosidine (=paromomycin) antimonial combinations, a pilot study in Bihar, India. Transactions of the Royal Society of Tropical Medicine and Hygiene 86; 615-616.

Theodos CM, Ribeiro JMC, Titus RG. (1991): Analysis of enhancing effect of sandfly saliva on leishmania infection in mice. Infection and Immunity 51; $1592-1598$.

Verrier RL, Lown B (1975): Sympathetic- parasympathetic interactions and ventricular electrical stability. In: Schwartz PJ, Brown AM, Malliani A, Zanchetti A (eds). Neural mechanisms in cardiac arrhythmias. New York, Raven Press p 75-86. 
Vianna G (1911): Sobre uma nova espécia de leishmania (nota preliminar). Brasil-Medico 25; 411.

Vianna G (1912): Tratamento da leishmaniose tegumentar por injeções intravenosas de tartar emético. Anias do 70 Congresso Brasileiro de Medicina e Cirurgia 4; 426-428.

Vianna G (1914): Sombre o tratamento de leishmaniose tegumentar. Anias Paulista de Medicina e Cirurgia 2; 167-169.

Viega JPR, Wolff ER, Sampaio, RN, Marsden PD (1983): Renal tubular dysfunction in patients with mucocutaneous leishmaniasis treated with pentavalent antimonials Lancet ii; 569.

Viera JB, Lacerda MM, Marsden PD (1990): National reporting of leishmaniasis: the Brazilian expirience. Parasitology Today 6; 399340.

Walton BC (1980): Evaluation of chemotherapy of American cutaneous leishmaniasis by the indirect fluorescent antibody test. American Journal of Tropical Medicine and Hygiene 29; 747-752.

Walton BC 1987): American cutaneous and mucocutaneous leishmaniasis. In: Peters W, Killick-Kendrick R (eds). The leishmaniases in biology and medicine, volume 2. London, Academic Press. p637-664.

Weigle KA, de Davalos M, Heredia P, Molineros R, Saravia NG, D'Alessandro A (1987): Diagnosis of cutaneous and mucocutaneous leishmaniasis in Colombia: A comparason of seven methods. American Journal of Tropical Medicine and Hygiene 42; 489-496.

Weigle KA, Santrich C, Martinez F, Valderrama L, Saravia NG (1993a): Epidemiology of cutaneous leishmaniasis in Colombia: a longitudinal study of the natural history, prevalence, and incidence of infection and clinical manifestations. Journal of infectious Diseases 168; 699-708.

Weigle KA, Santrich C, Martinez F, Valderrama L, Saravia NG (1993b): Epidemiology of cutaneous leishmaniasis in Colombia: enviromental and behavioral risk factors for infection, clinical manifestations, and pathogenicity. Journal of infectious Diseases 168; 709-714.

Weigle KA, Valderrama L, Arias AL, Santrica C, Saravia NG (1991): Leishmanin standardization and evaluation of safety, dose, storage, longevity of reaction and sensitization. American Journal of Tropical Medicine and Hygiene 44; 260-271.

Weinrauch L, Cawich F, Craig P, Sosa StJX, El-On J (1993): Topical treatment of New World leishmaniasis in Belize: A clinical study. Journal of the American Academy of Dermatology 29; 443-446. 
Weinrauch L, Katz M, El-On J (1987): Leishmania aethiopica: topical treatment with paromomycin and methylbenzethonium chloride ointment. Journal of the American Academy of Dermatology 16; 12681270.

WHO Expert Commitee (1984): The leishmaniases. Technical Report Series 701 Geneva, WHO.

WHO Expert Commitee (1990) Control of the leishmaniases. Technical Report Series 793 Geneva, WHO.

Wilson SM (1991): Nucleic acid techniques and the detection of parasitic diseases. Parasitology Today 7; 255-259.

Wirth D (1992): The diagnosis of leishmaniasis: current and future techniques. Proceedings of the 18th World Congress of Dermatology. New York June 1992.

Wirth DF, McMahon-Pratt D (1982): Rapid identification of leishmania species by specific hybridization of kinetoplast DNA in cutaneous lesions. Proceedings of the National Academy of Sciences of the United States of America 79; 6999-7003.

Wright JH (1903): Protozoa in a case of tropical ulcer (Dehli sore). Journal of Medical Research 10; 472-482.

Zuaneti G, Latini R, Neilson JMM, Schwartz PJ, Ewing DJ (1991): Heart rate variability in patients with cardiac arrhythmias: effect of anti-arrhythmic drugs. Journal of the American College of Cardiologists 17; 604-612. 
Appendix 1.1:

Data on 187 cases in which parasitological confirmation of the diagnosis was obtained

1.Number of lesions on each soldier

\begin{tabular}{cccc}
\hline No of lesions & All cases & $\frac{\text { Lbb cases }}{57(74 \%)}$ & $\frac{\text { Lmm cases }}{19(65.5 \%)}$ \\
\cline { 2 - 4 } 1 & $133(71 \%)$ & $13(16.9 \%)$ & $6(20.7 \%)$ \\
2 & $30(16 \%)$ & $5(6.5 \%)$ & $4(13.8 \%)$ \\
3 & $15(8 \%)$ & $1(1.3 \%)$ & 0 \\
5 & $7(3.7 \%)$ & 0 & 0 \\
12 & $1(0.5 \%)$ & $1(1.3 \%)$ & 0 \\
\hdashline & $1(0.5 \%)$ & -17 & 29 \\
\hline
\end{tabular}

\section{Site of lesion}

\begin{tabular}{|c|c|c|c|c|}
\hline Site & & All lesions & Lbb lesons & Lmm lesions \\
\hline & $\begin{array}{c}\text { Head \& neck } \\
\text { total }\end{array}$ & $73(25.8 \%)$ & $23(20 \%)$ & \\
\hline & nose & $2(0.7 \%)$ & 0 & $\begin{array}{r}20(46.5 \%) \\
1 \quad(2.3 \%)\end{array}$ \\
\hline & cheeks & $33(11.7 \%)$ & $13(11.4 \%)$ & $9(20.9 \%)$ \\
\hline & & $2(0.7 \%)$ & & $2(4.7 \%)$ \\
\hline & chin & $5(1.8 \%)$ & $3(2.6 \%)$ & \\
\hline & ears & 11 (3.9\%) & $6 \quad(5.2 \%)$ & $2(4.7 \%)$ \\
\hline & eyelid & $1 \quad(0.35 \%)$ & 0 & 1 (2.3\%) \\
\hline & forehead & $3(1.1 \%)$ & 0 & $1(2.3 \%)$ \\
\hline & scalp & $1 \quad(0.35 \%)$ & 0 & \\
\hline & neck & $15(5.3 \%)$ & $1 \quad(0.8 \%)$ & $4 \quad(9.3 \%)$ \\
\hline & Trunk & $44(15.5 \%)$ & $16(14 \%)$ & $8(18.6 \%)$ \\
\hline & Arms & $33(11.7 \%)$ & $12(10.5 \%)$ & $6(14 \%)$ \\
\hline & Hands \& wrists & $72(25.4 \%)$ & $27(23.7 \%)$ & $4(9.3 \%)$ \\
\hline & Legs & $56(19.8 \%)$ & $32(28 \%)$ & $5(11.6 \%)$ \\
\hline & Unknown & $5(1.8 \%)$ & $4(3.5 \%)$ & 0 \\
\hline & & 283 lesions & 114 lesions & 43 lesions \\
\hline
\end{tabular}




\section{Size of lesions}

\begin{tabular}{|c|c|c|c|}
\hline Site of lesion & All cases & $\underline{\text { Lbb cases }}$ & $\underline{\mathrm{Lmm} \text { cases }}$ \\
\hline $\begin{array}{l}\text { Head \&neck } \\
\text { total } \\
\text { nose } \\
\text { cheeks } \\
\text { lips } \\
\text { chin } \\
\text { ears } \\
\text { eyelids } \\
\text { forehead } \\
\text { scalp } \\
\text { neck } \\
\text { Trunk } \\
\text { Arms } \\
\text { Hands \& wrists } \\
\text { Legs } \\
\text { Unknown }\end{array}$ & $\begin{array}{l}11.5(44 / 73) \\
11.0(1 / 2) \\
11.0(19 / 33) \\
14.0(1 / 2) \\
16.0(5 / 5) \\
14.4(5 / 11) \\
--(0 / 1) \\
7.5(2 / 3) \\
15.0(1 / 1) \\
9.2(10 / 15) \\
12.0(34 / 44) \\
13.8(29 / 33) \\
16.1(52 / 72) \\
16.2(43 / 56) \\
25.0(1 / 5)\end{array}$ & $\begin{array}{c}14.0(16 / 23) \\
--(0 / 0) \\
11.6(8 / 13) \\
--(0 / 0) \\
21.7(3 / 3) \\
13.0(4 / 6) \\
(0 / 0) \\
--(0 / 0) \\
--(0 / 0) \\
14.0(1 / 1) \\
14.0(14 / 16) \\
17.1(11 / 12) \\
19.8(22 / 27) \\
17.7(27 / 32) \\
25.0(1 / 4)\end{array}$ & $\begin{array}{c}8.2(13 / 20) \\
11.0(1 / 1) \\
8.3(7 / 9) \\
14.0(1 / 2) \\
--(0 / 0) \\
-\quad(0 / 2) \\
--(0 / 0) \\
5.0(1 / 1) \\
-\overline{(0 / 0)} \\
10.3(3 / 4) \\
12.8(8 / 8) \\
14.0(6 / 6) \\
16.3(3 / 4) \\
15.0(5 / 5) \\
--(0 / 0)\end{array}$ \\
\hline $\begin{array}{l}\text { mean size } \\
\text { sd } \\
\% \text { known }\end{array}$ & $\begin{array}{l}14.4 \mathrm{~mm} \\
9.3 \\
71.7 \%\end{array}$ & $\begin{array}{l}17.0 \mathrm{~mm} \\
10.3 \\
79.8 \%\end{array}$ & $\begin{array}{l}12.3 \mathrm{~mm} \\
6.5 \\
81.4 \%\end{array}$ \\
\hline \multicolumn{4}{|c|}{$\begin{array}{l}\text { Table shows the mean diameter of ulcer in } \mathrm{mm} \text { (longest axis). (The proportion of } \\
\text { lesions in which the size was known is shown in brackets). }\end{array}$} \\
\hline \multicolumn{4}{|c|}{ 4. Duration of lesion before treatment started (weeks) } \\
\hline & All cases & $\underline{\text { Lbb cases }}$ & $\underline{\text { Lmm cases }}$ \\
\hline $\begin{array}{l}\text { mean } \\
\text { sd }\end{array}$ & $\begin{array}{l}9.9 \\
6.7\end{array}$ & $\begin{array}{l}8.8 \\
4.5\end{array}$ & $\begin{array}{l}12.5 \\
9.0\end{array}$ \\
\hline$\%$ known & $\begin{array}{l}89.9 \% \\
(168 / 187)\end{array}$ & $\begin{array}{l}85.7 \% \\
(66 / 77)\end{array}$ & $\begin{array}{l}93.1 \% \\
(27 / 29)\end{array}$ \\
\hline
\end{tabular}


Appendix 1.2:

Comparason of clinical features of $\mathbf{1 8 7}$ parasitologically confirmed cases with all 306 cases in which a clinical diagnosis of $\mathrm{CL}$ was made.

1. Number of lesions on each soldier

\begin{tabular}{ccc}
\hline No of lesions & $\frac{\text { Parasite shown }}{133(71.0 \%)}$ & $\frac{\text { All cases }}{213(69.6 \%)}$ \\
\hline 1 & $30(16 \%)$ & $50(16.3 \%)$ \\
3 & $15(8 \%)$ & $21(6.7 \%)$ \\
4 & $7(3.7 \%)$ & $10(3.3 \%)$ \\
5 & $1(0.5 \%)$ & $1(0.3 \%)$ \\
6 & 0 & $6(1.9 \%)$ \\
8 & 0 & $3(1.0 \%)$ \\
9 & 0 & $1(0.3 \%)$ \\
12 & $1(0.5 \%)$ & $1(0.3 \%)$ \\
Total & 187 & $-\cdots$ \\
\hline
\end{tabular}

\section{Site of lesions}

\begin{tabular}{ccr}
\hline Head \& neck & Parasite shown & \multicolumn{1}{c}{ All cases } \\
total & $73(25.8 \%)$ & $122(24.3 \%)$ \\
nose & $2(0.7 \%)$ & $6(1.2 \%)$ \\
cheeks & $33(11.7 \%)$ & $44(8.8 \%)$ \\
lip & $2(0.7 \%)$ & $6(1.2 \%)$ \\
chin & $5(1.8 \%)$ & $8(1.6 \%)$ \\
ears & $11(3.9 \%)$ & $20(4.0 \%)$ \\
eyelids & $1(0.35 \%)$ & $10(2.0 \%)$ \\
forehead & $3(1.1 \%)$ & $5(1.0 \%)$ \\
scalp & $1(0.35 \%)$ & 1 \\
neck & $15(5.3 \%)$ & $22(4.4 \%)$ \\
Trunk & $44(15.5 \%)$ & $49(9.8 \%)$ \\
Arms & $33(11.7 \%)$ & $51(10.2 \%)$ \\
Hands \& wrists & $72(25.4 \%)$ & $132(26.2 \%)$ \\
Legs & $56(19.8 \%)$ & $142(28.2 \%)$ \\
Unknown & $5(1.8 \%)$ & $6(1.1 \%)$ \\
Total & 283 lesions &...-- \\
& & 624 lesions \\
\hline
\end{tabular}




\section{Size of lesions.}

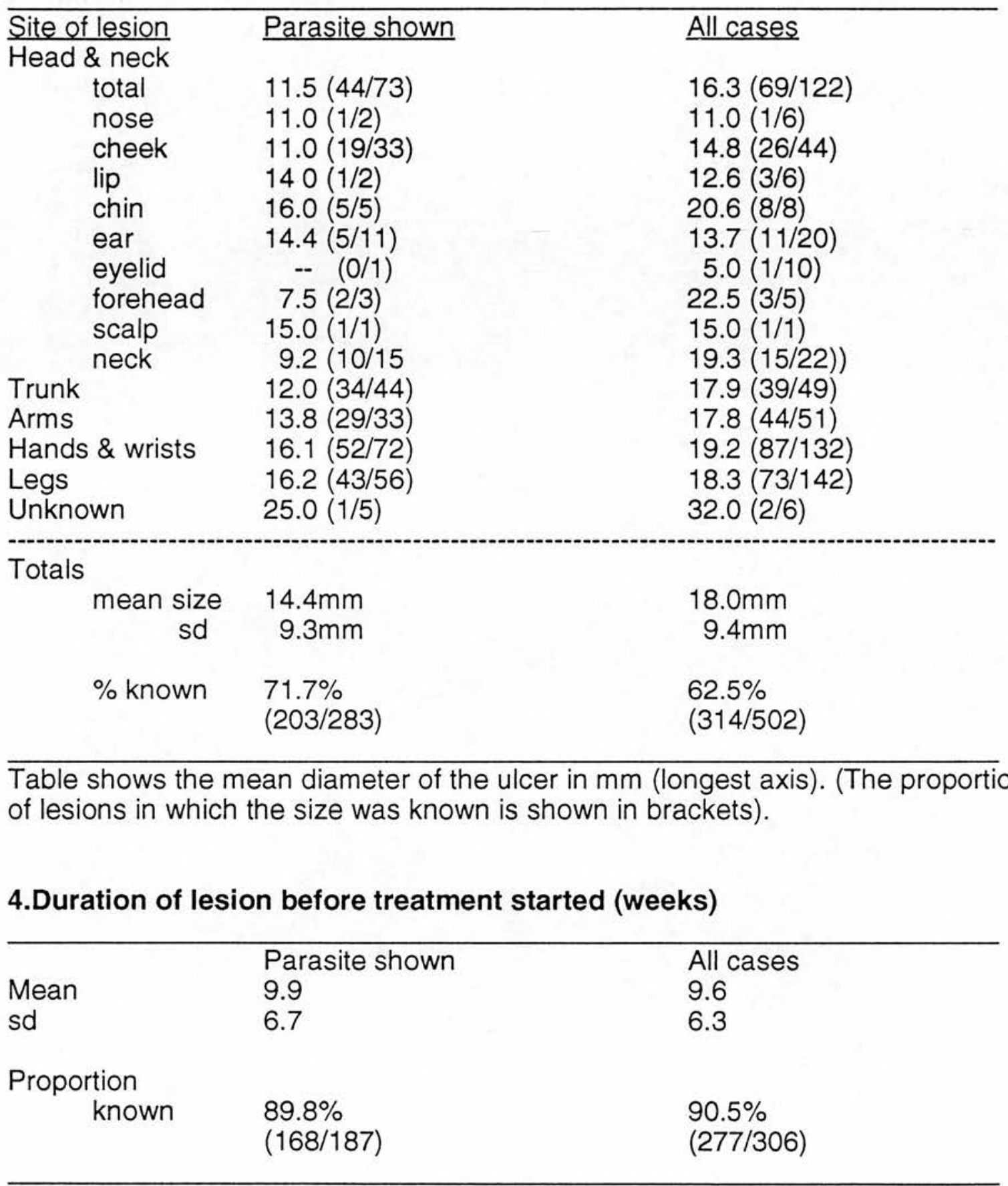


Appendix 1.3

Table 1 Haematological and biochemical changes during treatment with sodium stibogluconate $600 \mathrm{mg} /$ day for 10 days (total $6 \mathrm{~g}$ ).

The mean ( $\mathrm{x})$, standard error (se) and the number of soldiers in which it was available $(n)$ are shown. $\left({ }^{*}=\right.$ significant change $\left.P<0.05\right)$.

\begin{tabular}{|c|c|c|c|c|c|}
\hline & $\begin{array}{l}\text { Normal } \\
\text { range }\end{array}$ & & $\begin{array}{l}\text { Pre- } \\
\text { treatment }\end{array}$ & $\begin{array}{l}\text { Post- } \\
\text { treatment }\end{array}$ & $\begin{array}{l}2 \text { Weeks after } \\
\text { treatment }\end{array}$ \\
\hline $\begin{array}{l}\text { WBC } \\
\times 10^{9} / 1\end{array}$ & $4-11$ & $\begin{array}{l}x \\
\text { sd } \\
n\end{array}$ & $\begin{array}{l}6.72 \\
2.24 \\
36\end{array}$ & $\begin{array}{ll}5.90^{*} & 7.19 \\
1.38 & \\
35 & \end{array}$ & $\begin{array}{l}2.28 \\
26\end{array}$ \\
\hline $\mathrm{Hb} \mathrm{g} / \mathrm{l}$ & $13.5-18$ & $\begin{array}{l}x \\
\text { sd } \\
n\end{array}$ & $\begin{array}{l}15.6 \\
1.24 \\
36\end{array}$ & $\begin{array}{l}15.4 \\
1.08 \\
35\end{array}$ & $\begin{array}{l}15.4 \\
1.19 \\
26\end{array}$ \\
\hline $\begin{array}{l}\text { Urea } \\
\mathrm{mmol} / \mathrm{l}\end{array}$ & $3-6.5$ & $\begin{array}{l}x \\
\text { sd } \\
n\end{array}$ & $\begin{array}{l}4.75 \\
0.90 \\
36\end{array}$ & $\begin{array}{l}4.35^{\star} \\
1.08 \\
36\end{array}$ & $\begin{array}{l}4.33^{\star} \\
0.73 \\
27\end{array}$ \\
\hline$\underset{I U / I}{\text { ALK } P}$ & $36-92$ & $\begin{array}{l}x \\
\text { sd } \\
n\end{array}$ & $\begin{array}{l}73.0 \\
25.7 \\
36\end{array}$ & $\begin{array}{l}74.7 \\
19.6 \\
36\end{array}$ & $\begin{array}{l}81.7^{\star} \\
31.6 \\
26\end{array}$ \\
\hline $\begin{array}{l}\text { GGT } \\
\mathrm{mmol} / \mathrm{l}\end{array}$ & $11-54$ & $\begin{array}{l}x \\
\text { sd } \\
n\end{array}$ & $\begin{array}{l}23.8 \\
26.6 \\
36\end{array}$ & $\begin{array}{l}26.8 \\
17.9 \\
36\end{array}$ & $\begin{array}{l}28.3 \\
25.8 \\
26\end{array}$ \\
\hline $\begin{array}{l}\text { ALT } \\
\mathrm{mmol} /\end{array}$ & $6-37$ & $\begin{array}{l}x \\
\text { sd } \\
n\end{array}$ & $\begin{array}{l}21.8 \\
20.0 \\
36\end{array}$ & $\begin{array}{l}84.6^{*} \\
70.0 \\
36\end{array}$ & $\begin{array}{l}31.7^{\star} \\
37.2 \\
26\end{array}$ \\
\hline $\begin{array}{l}\text { AST } \\
\mathrm{mmol} / \mathrm{l}\end{array}$ & $10-30$ & $\begin{array}{l}x \\
\text { sd } \\
n\end{array}$ & $\begin{array}{l}24.9 \\
17.2 \\
36\end{array}$ & $\begin{array}{l}54.3^{*} \\
38.0 \\
36\end{array}$ & $\begin{array}{l}29.8 \\
26.4 \\
26\end{array}$ \\
\hline $\begin{array}{l}\text { Creatine } \\
\text { Kinase } \\
\text { IU/I }\end{array}$ & $36-188$ & $\begin{array}{l}x \\
\text { sd } \\
n\end{array}$ & $\begin{array}{c}161 \\
81.2 \\
21\end{array}$ & $\begin{array}{l}107^{\star} \\
55.5 \\
21\end{array}$ & $\begin{array}{c}141 \\
94.1 \\
19\end{array}$ \\
\hline $\begin{array}{l}\mathrm{LDH} \\
\mathrm{IU} / \mathrm{I}\end{array}$ & $190-340$ & $\begin{array}{l}x \\
\text { sd } \\
n\end{array}$ & $\begin{array}{l}273 \\
108 \\
21\end{array}$ & $\begin{array}{c}197^{*} \\
84 \\
21\end{array}$ & $\begin{array}{c}264 \\
67 \\
19\end{array}$ \\
\hline
\end{tabular}




\section{Appendix 1.3}

Table 2. Haematological and biochemical changes during treatment with sodium stibogluconate $600 \mathrm{mg}$ twice daily for 14 days (total $16.8 \mathrm{~g}$ ).

Abbreviations as for table 1.

\begin{tabular}{|c|c|c|c|c|c|}
\hline & $\begin{array}{l}\text { Normal } \\
\text { range }\end{array}$ & & $\begin{array}{l}\text { Pre- } \\
\text { treatment }\end{array}$ & $\begin{array}{l}\text { Post- } \\
\text { treatment }\end{array}$ & $\begin{array}{l}2 \text { Weeks after } \\
\text { treatment }\end{array}$ \\
\hline $\begin{array}{l}\text { WBC } \\
\times 10^{9 / I}\end{array}$ & $4.5-6.5$ & $\begin{array}{l}x \\
s d \\
n\end{array}$ & $\begin{array}{l}6.13 \\
1.04 \\
23\end{array}$ & $\begin{array}{l}5.13^{\star} \\
1.20 \\
23\end{array}$ & $\begin{array}{l}7.04^{\star} \\
1.38 \\
12\end{array}$ \\
\hline $\begin{array}{l}\mathrm{Hb} \\
\mathrm{g} / \mathrm{l}\end{array}$ & $13-18$ & $\begin{array}{l}x \\
s d \\
n\end{array}$ & $\begin{array}{c}14.9 \\
0.84 \\
23\end{array}$ & $\begin{array}{c}14.9 \\
0.94 \\
23\end{array}$ & $\begin{array}{l}14.9 \\
1.39 \\
11\end{array}$ \\
\hline $\begin{array}{l}\text { Urea } \\
\text { mmol/l }\end{array}$ & $3.1-6.1$ & $\begin{array}{l}x \\
s d \\
n\end{array}$ & $\begin{array}{l}4.73 \\
1.11 \\
24\end{array}$ & $\begin{array}{l}4.58 \\
1.24 \\
24\end{array}$ & $\begin{array}{l}5.33 \\
2.17 \\
12\end{array}$ \\
\hline $\begin{array}{c}\text { ALK P } \\
\text { IU/I }\end{array}$ & $75-275$ & $\begin{array}{l}x \\
s d \\
n\end{array}$ & $\begin{array}{c}167 \\
40 \\
24\end{array}$ & $\begin{array}{l}254^{*} \\
167 \\
24\end{array}$ & $\begin{array}{l}226 \\
84 \\
20\end{array}$ \\
\hline $\begin{array}{l}\text { GGT } \\
\mathrm{mmol} / \mathrm{l}\end{array}$ & $3-45$ & $\begin{array}{l}x \\
s d \\
n\end{array}$ & $\begin{array}{l}28.7 \\
14.9 \\
24\end{array}$ & $\begin{array}{l}53.2^{\star} \\
48.7 \\
24\end{array}$ & $\begin{array}{l}42.5^{\star} \\
24.7 \\
20\end{array}$ \\
\hline $\begin{array}{l}\mathrm{ALT} \\
\mathrm{mmol} / \mathrm{l}\end{array}$ & $4-40$ & $\begin{array}{l}x \\
\text { sd } \\
n\end{array}$ & $\begin{array}{l}25.3 \\
14.4 \\
24\end{array}$ & $\begin{array}{l}282^{*} \\
382 \\
24\end{array}$ & $\begin{array}{l}66.6^{\star} \\
64.1 \\
20\end{array}$ \\
\hline $\begin{array}{l}\mathrm{AST} \\
\mathrm{mmol} / \mathrm{l}\end{array}$ & $11-45$ & $\begin{array}{l}x \\
\text { sd } \\
n\end{array}$ & $\begin{array}{c}22.8 \\
9.8 \\
24\end{array}$ & $\begin{array}{l}178^{\star} \\
249 \\
24\end{array}$ & $\begin{array}{l}50.4^{\star} \\
50.9 \\
20\end{array}$ \\
\hline $\begin{array}{c}\text { Creatine } \\
\text { Kinase } \\
\text { IU/I }\end{array}$ & $16-300$ & $\begin{array}{l}x \\
s d \\
n\end{array}$ & $\begin{array}{c}163 \\
62.6 \\
22\end{array}$ & $\begin{array}{l}112^{*} \\
129 \\
19\end{array}$ & $\begin{array}{l}209 \\
169 \\
14\end{array}$ \\
\hline $\begin{array}{l}\mathrm{LDH} \\
\mathrm{IU} / \mathrm{I}\end{array}$ & $105-195$ & $\begin{array}{l}x \\
s d \\
n\end{array}$ & $\begin{array}{l}200 \\
43.9 \\
22\end{array}$ & $\begin{array}{l}213 \\
73.9 \\
19\end{array}$ & $\begin{array}{c}207 \\
77.2 \\
13\end{array}$ \\
\hline
\end{tabular}




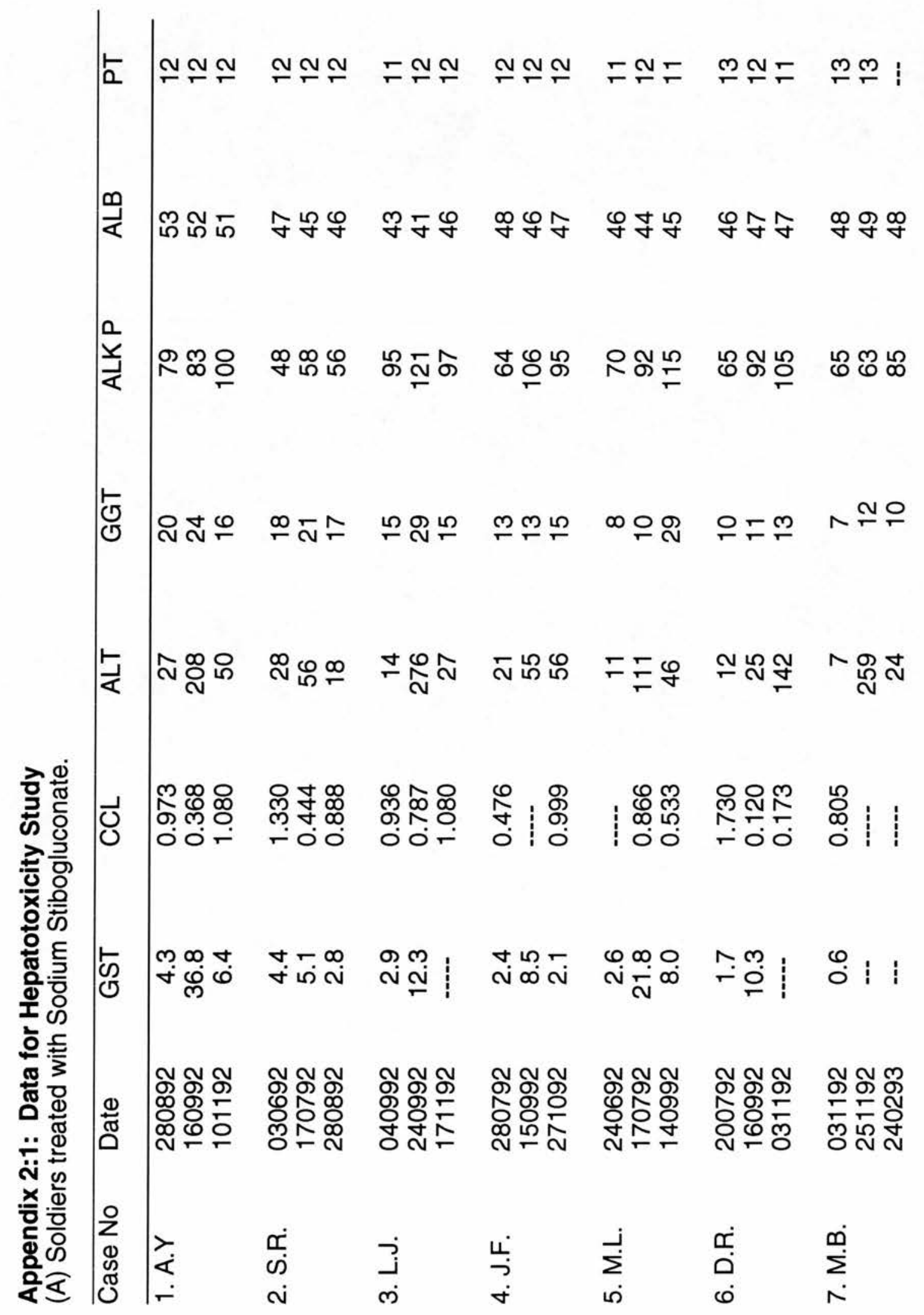




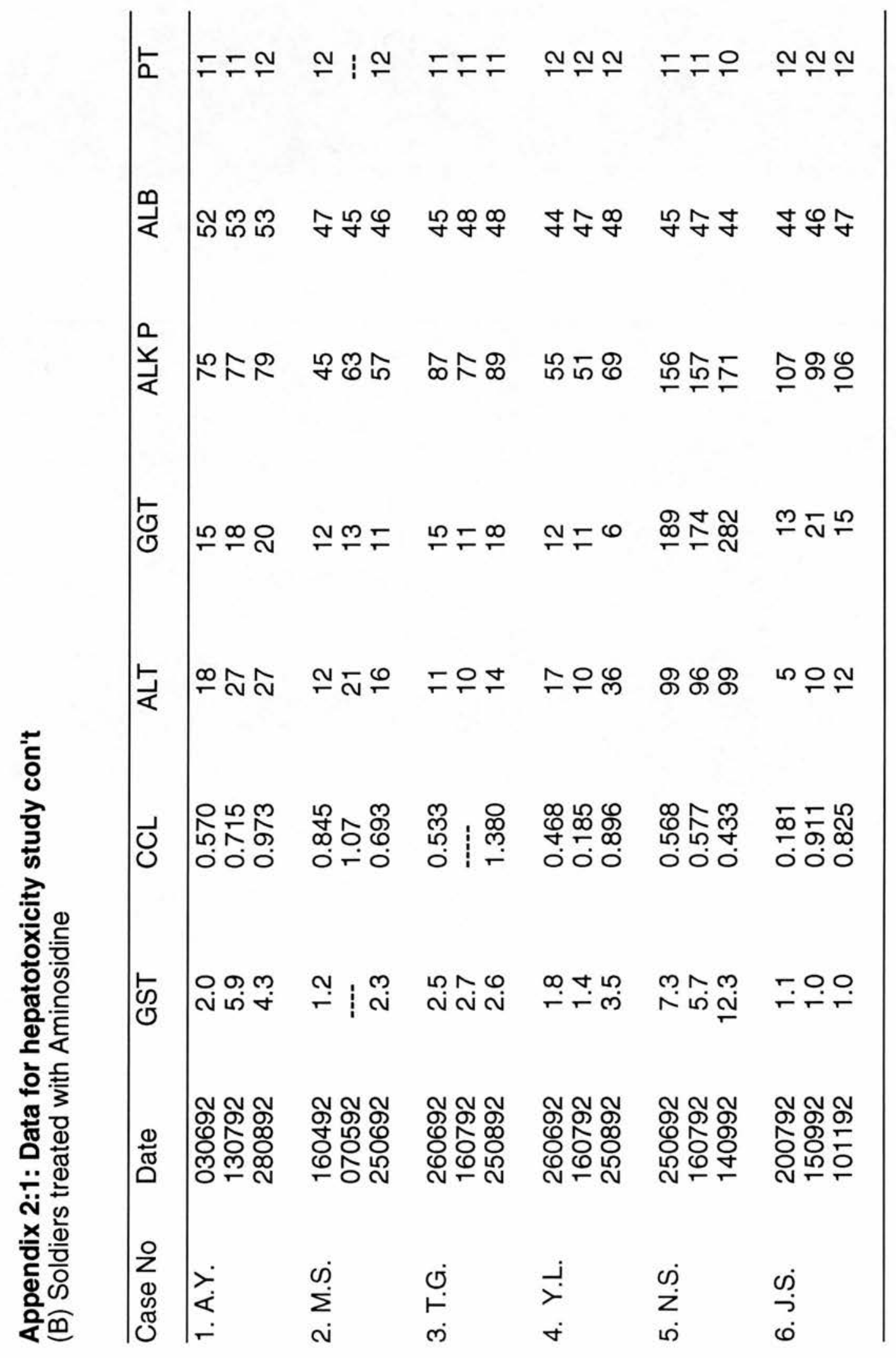




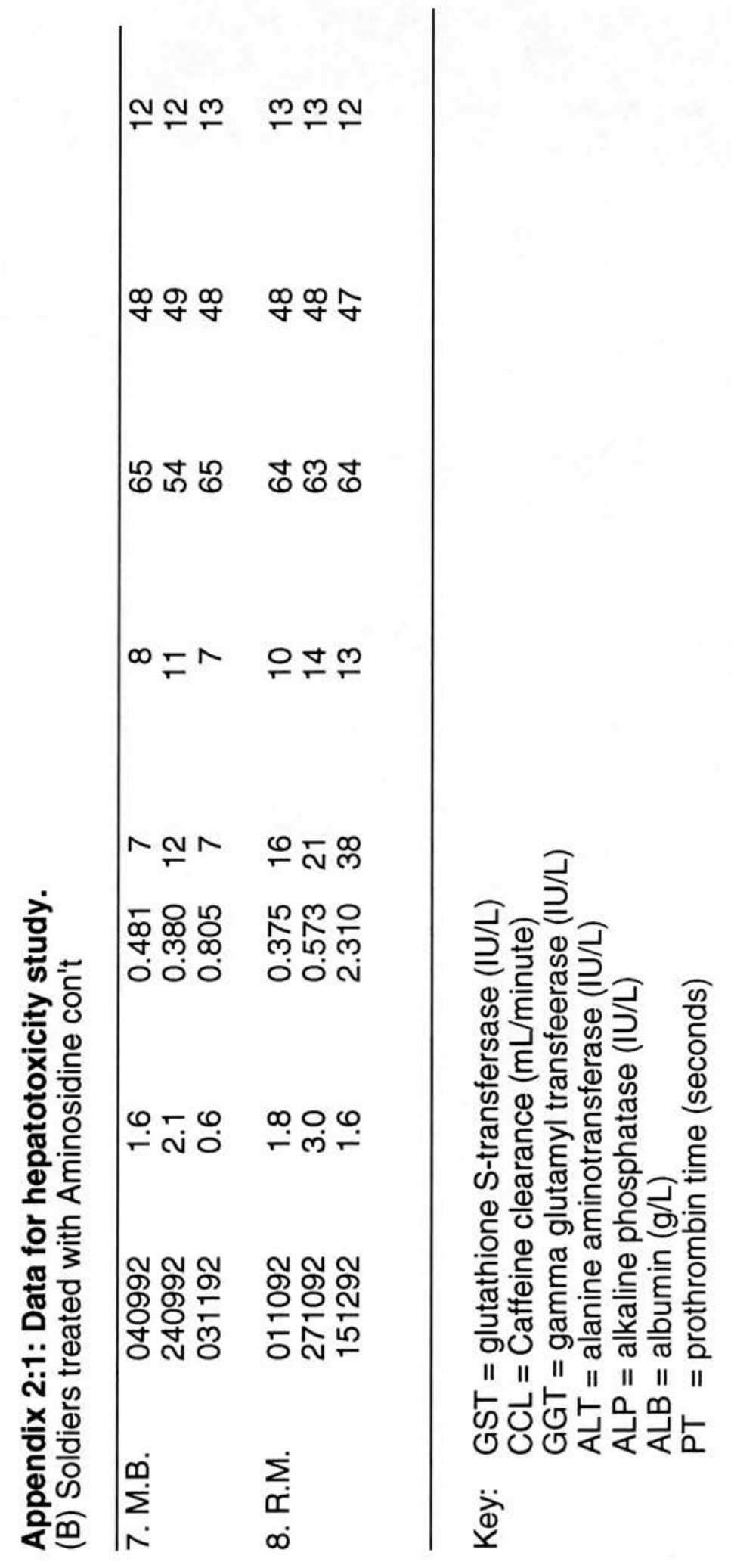




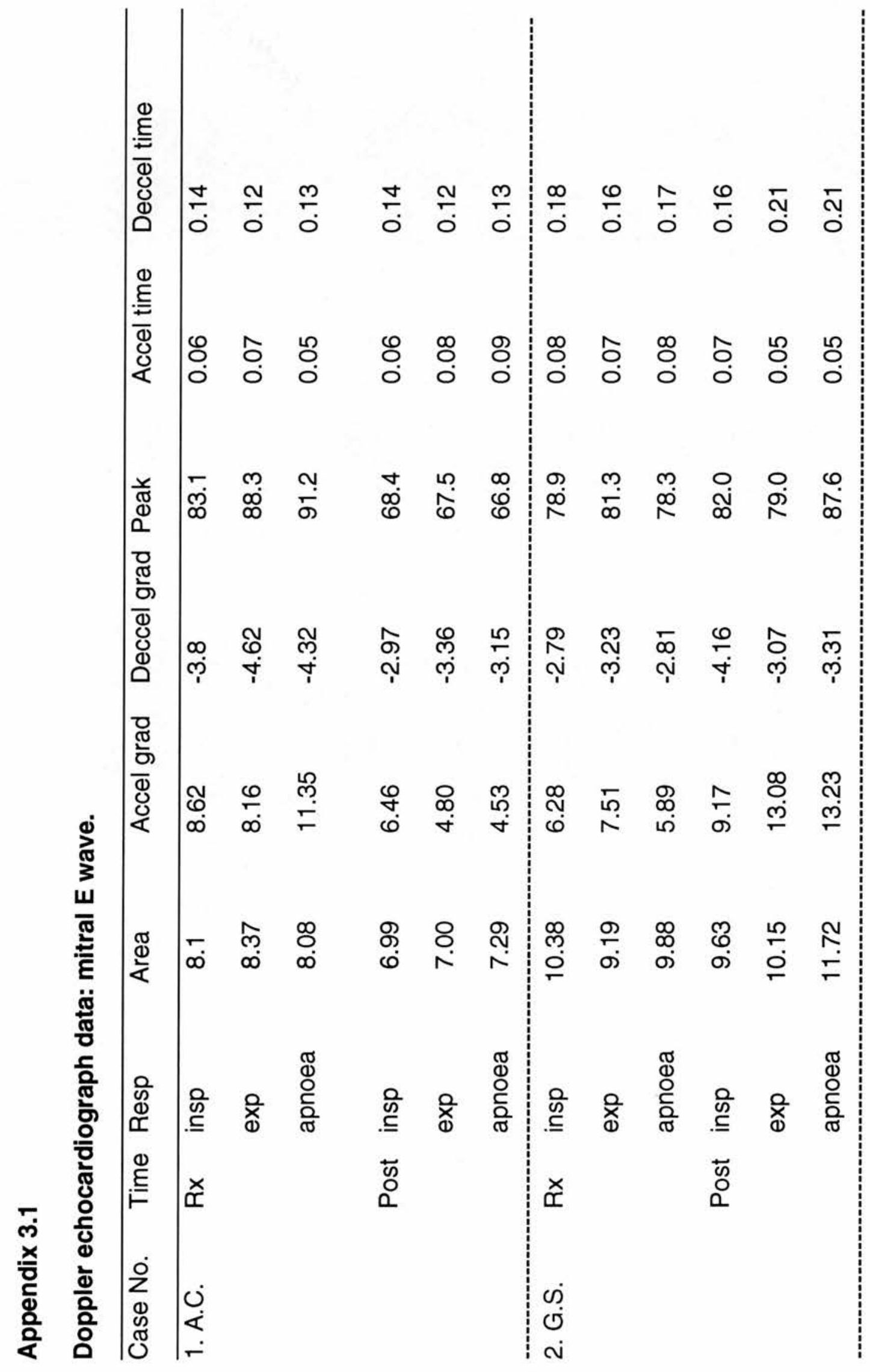




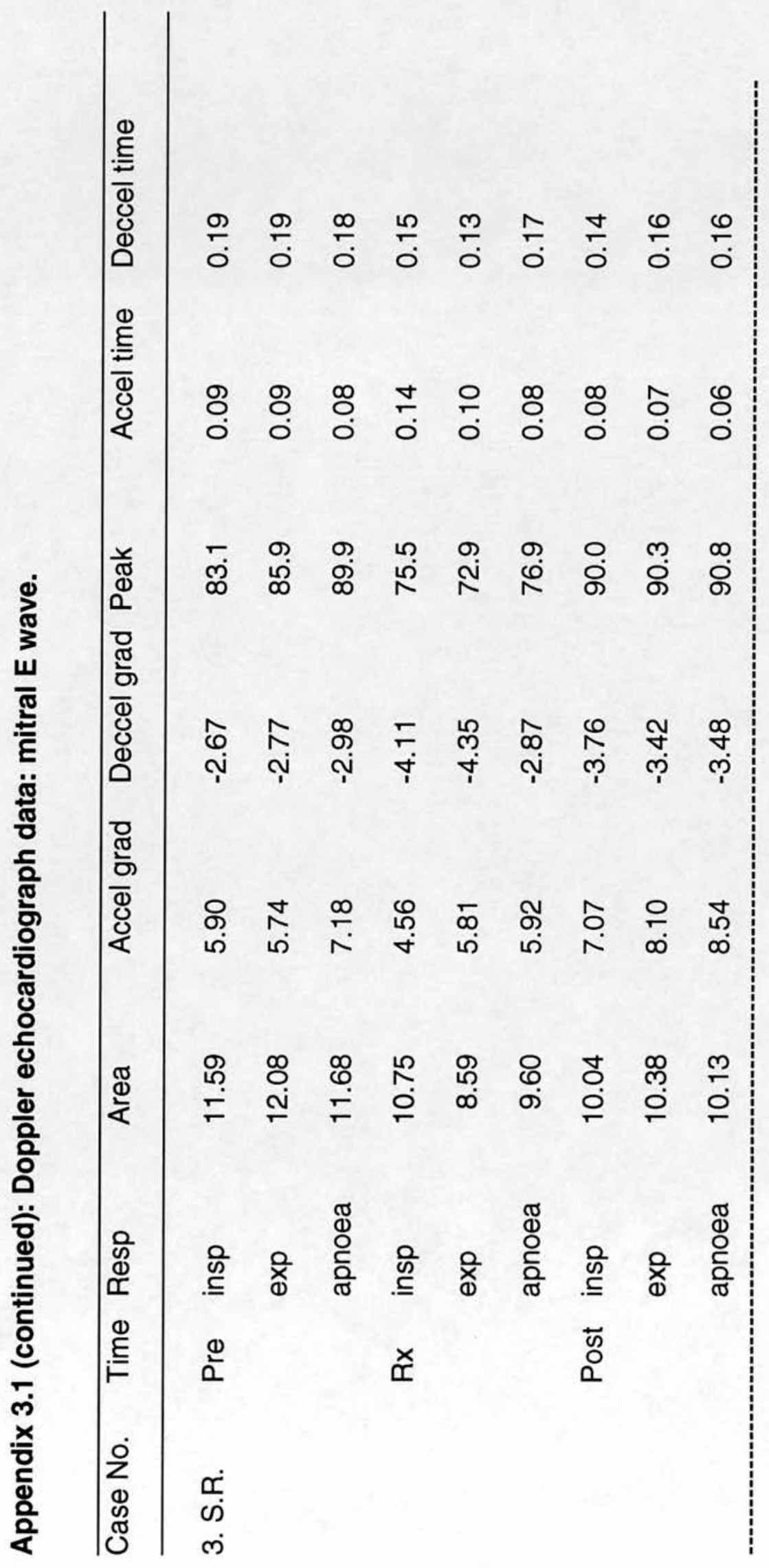




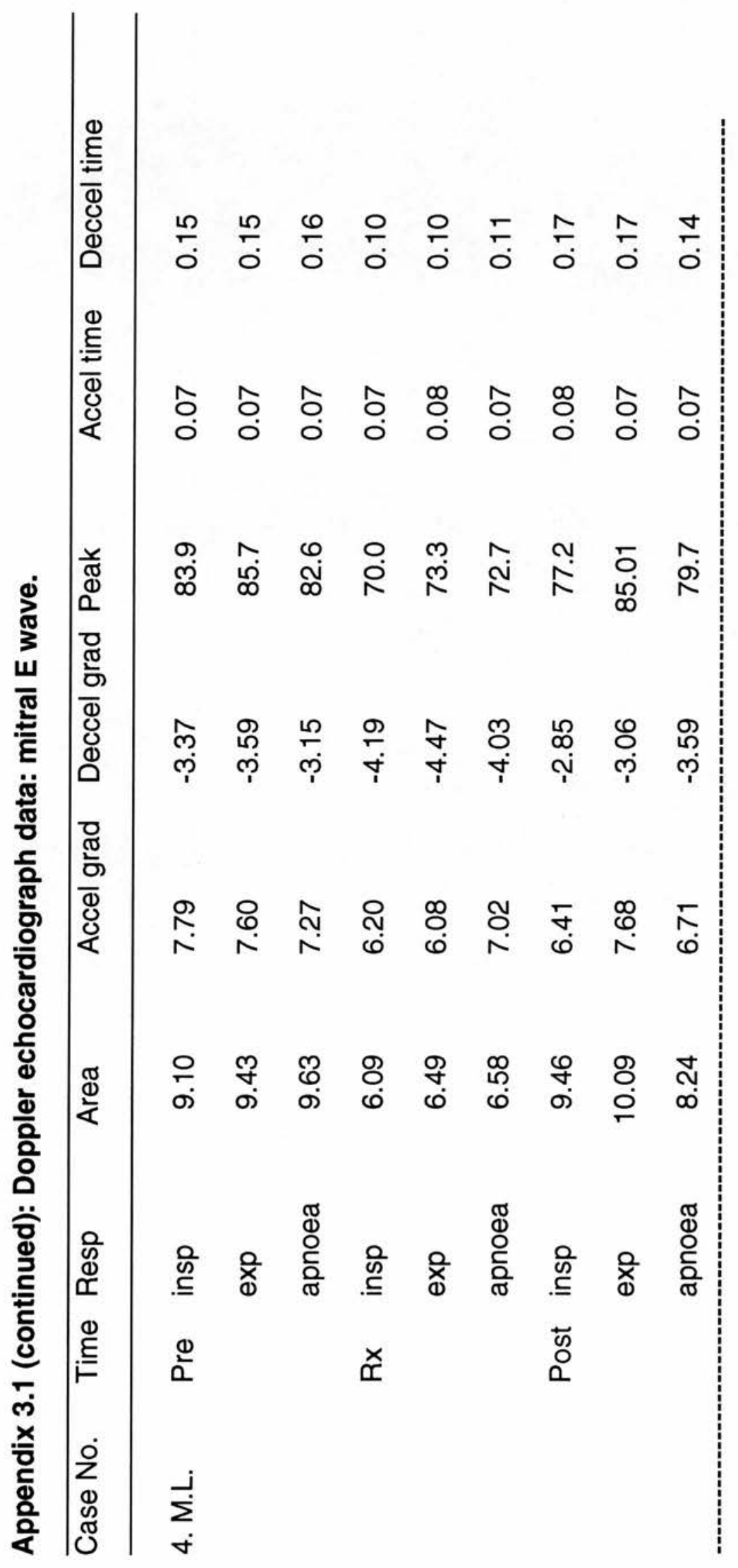




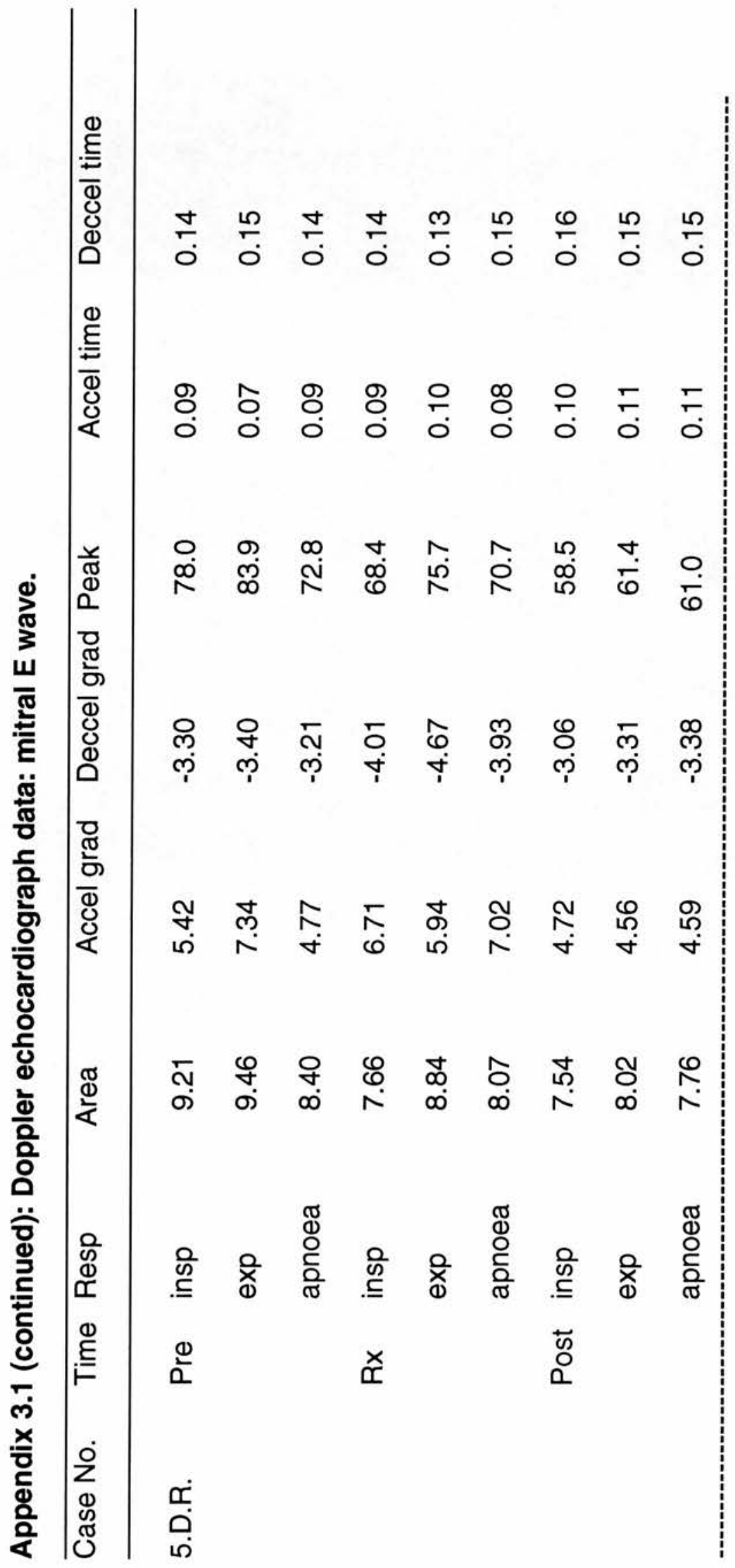




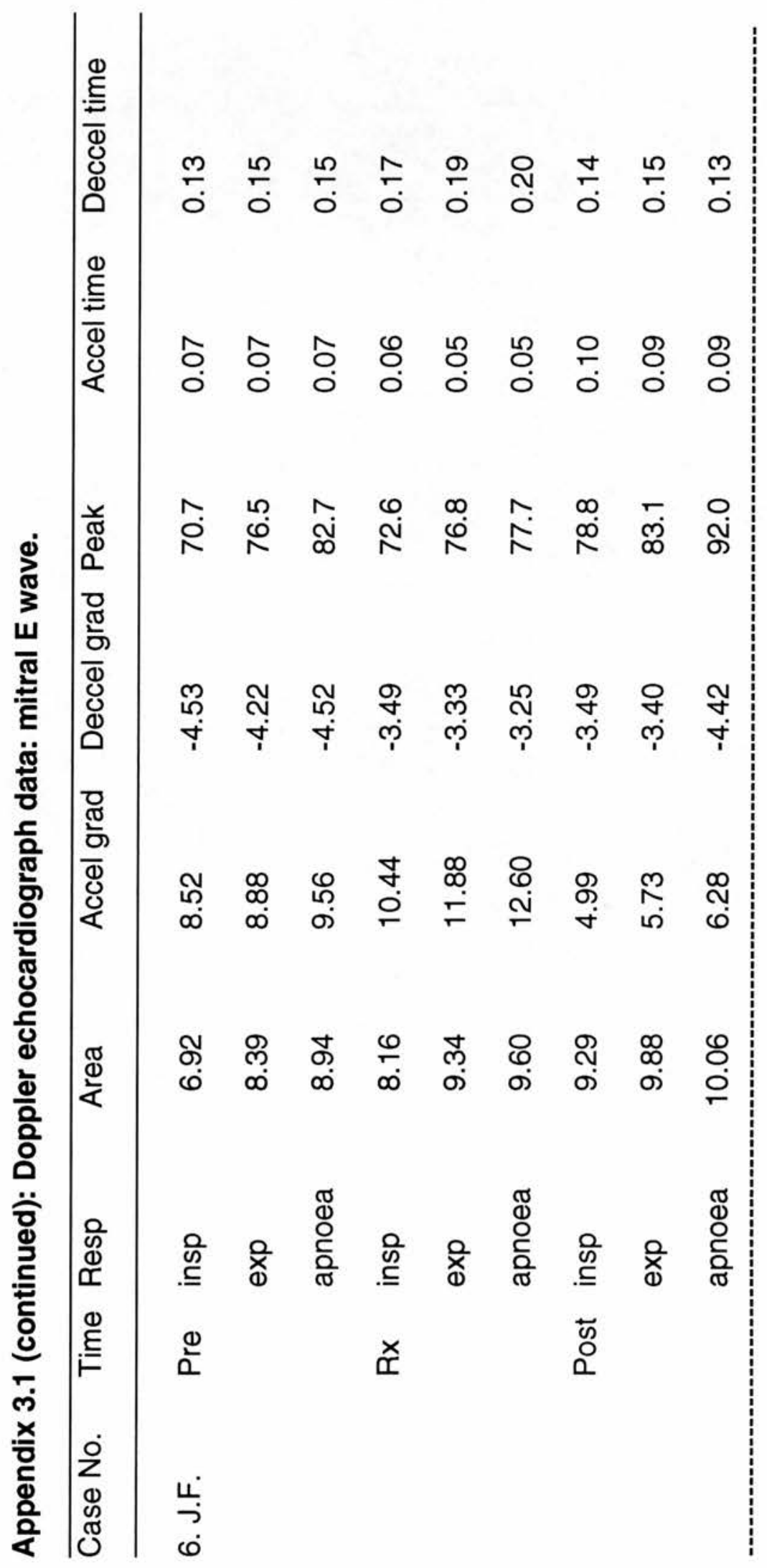




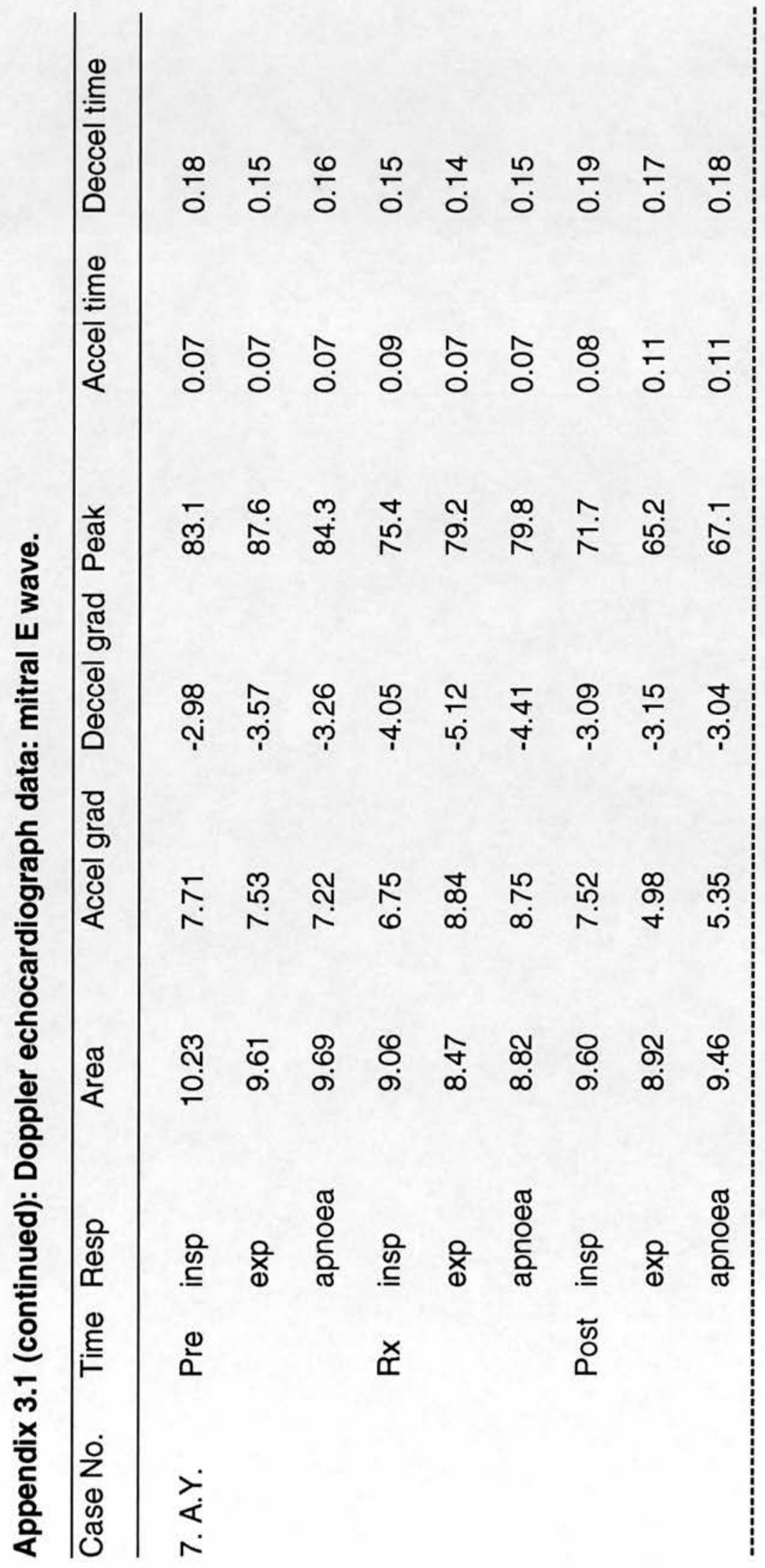









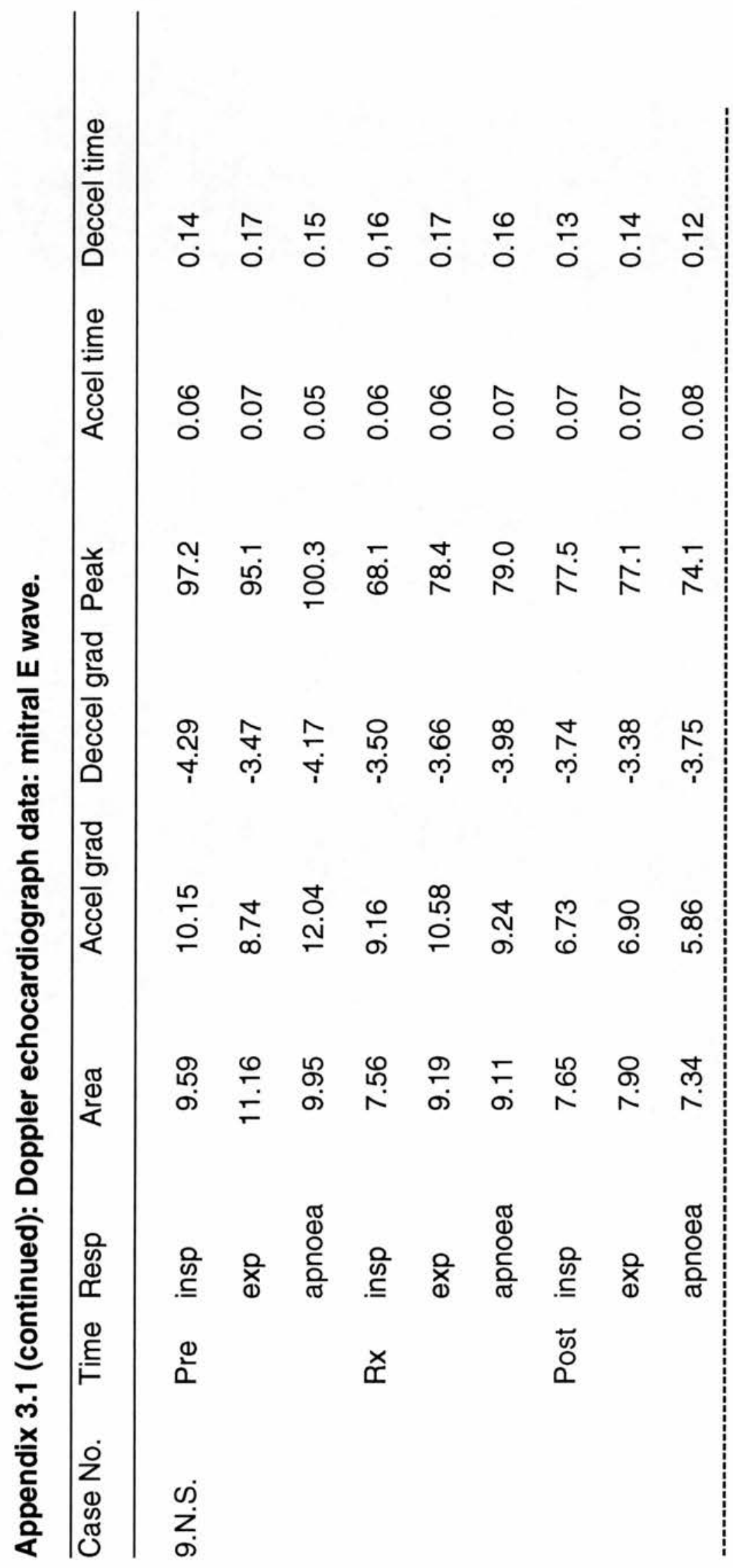




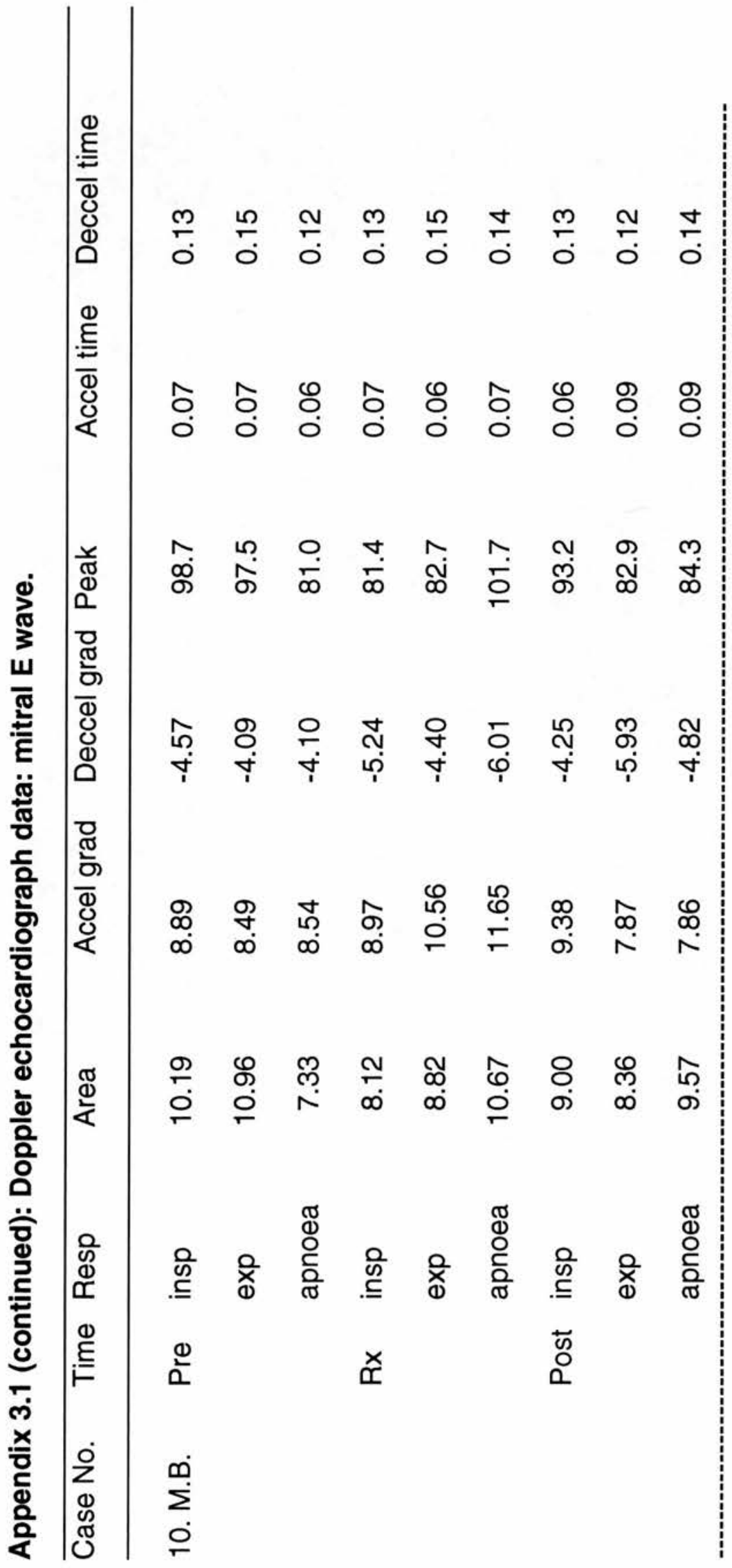




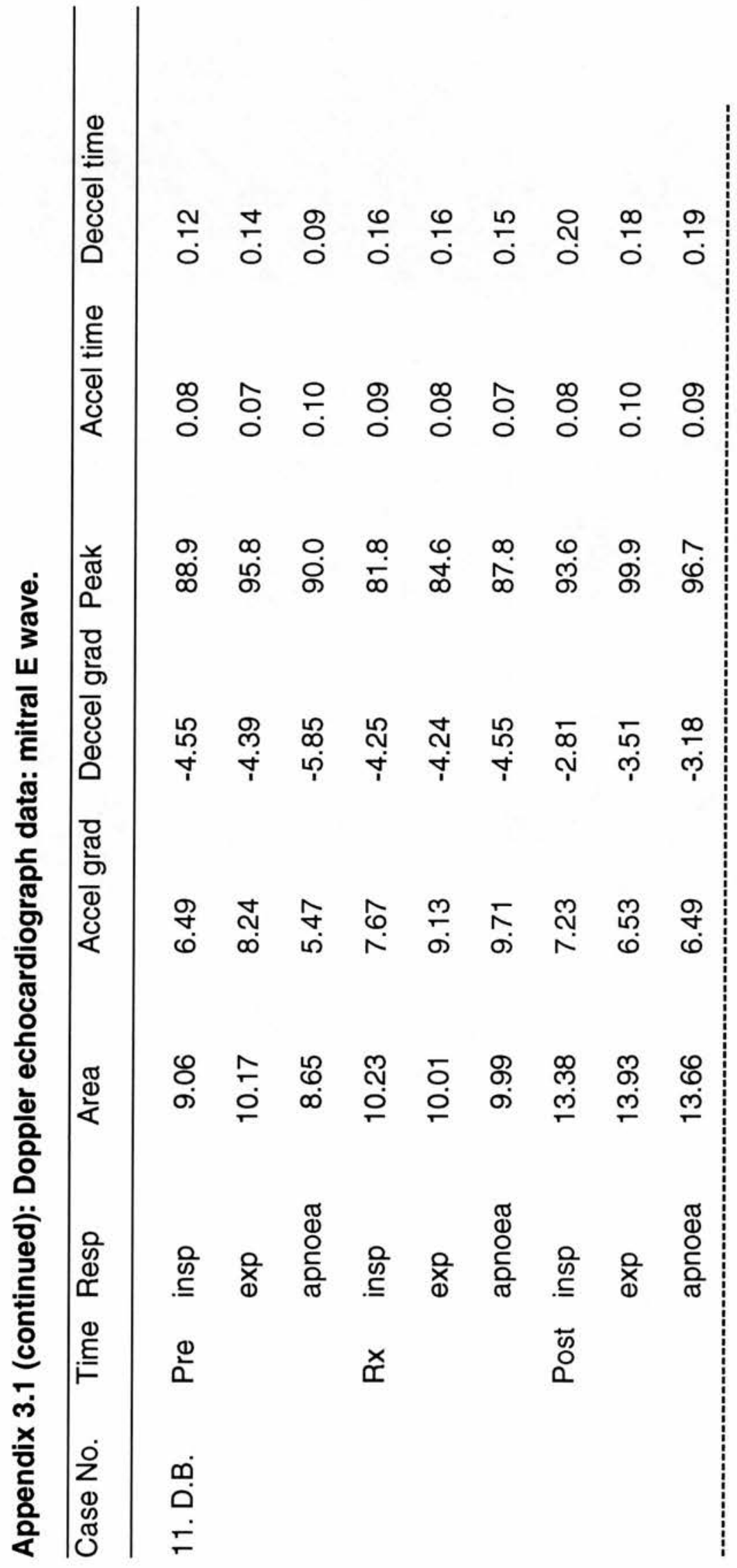




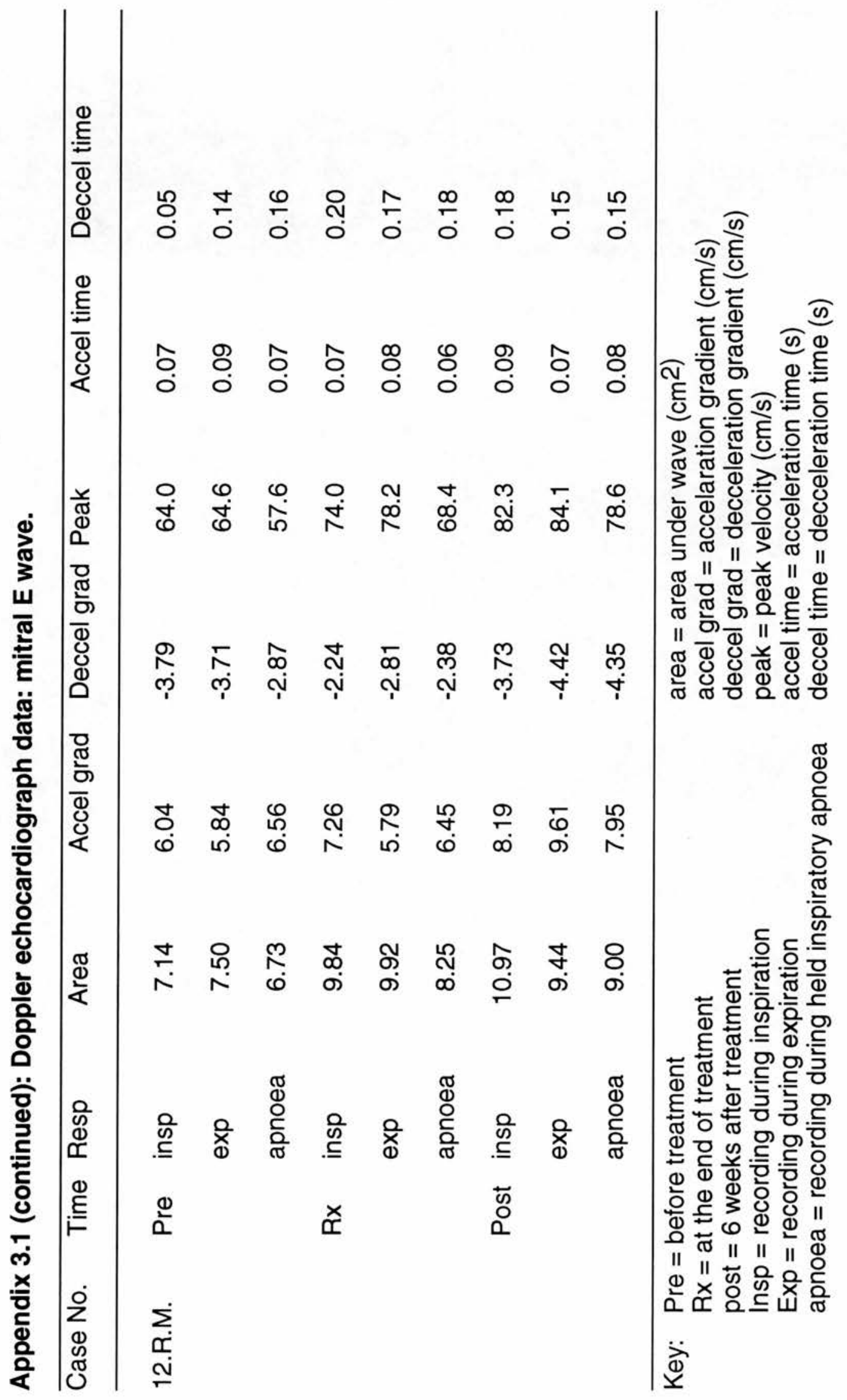




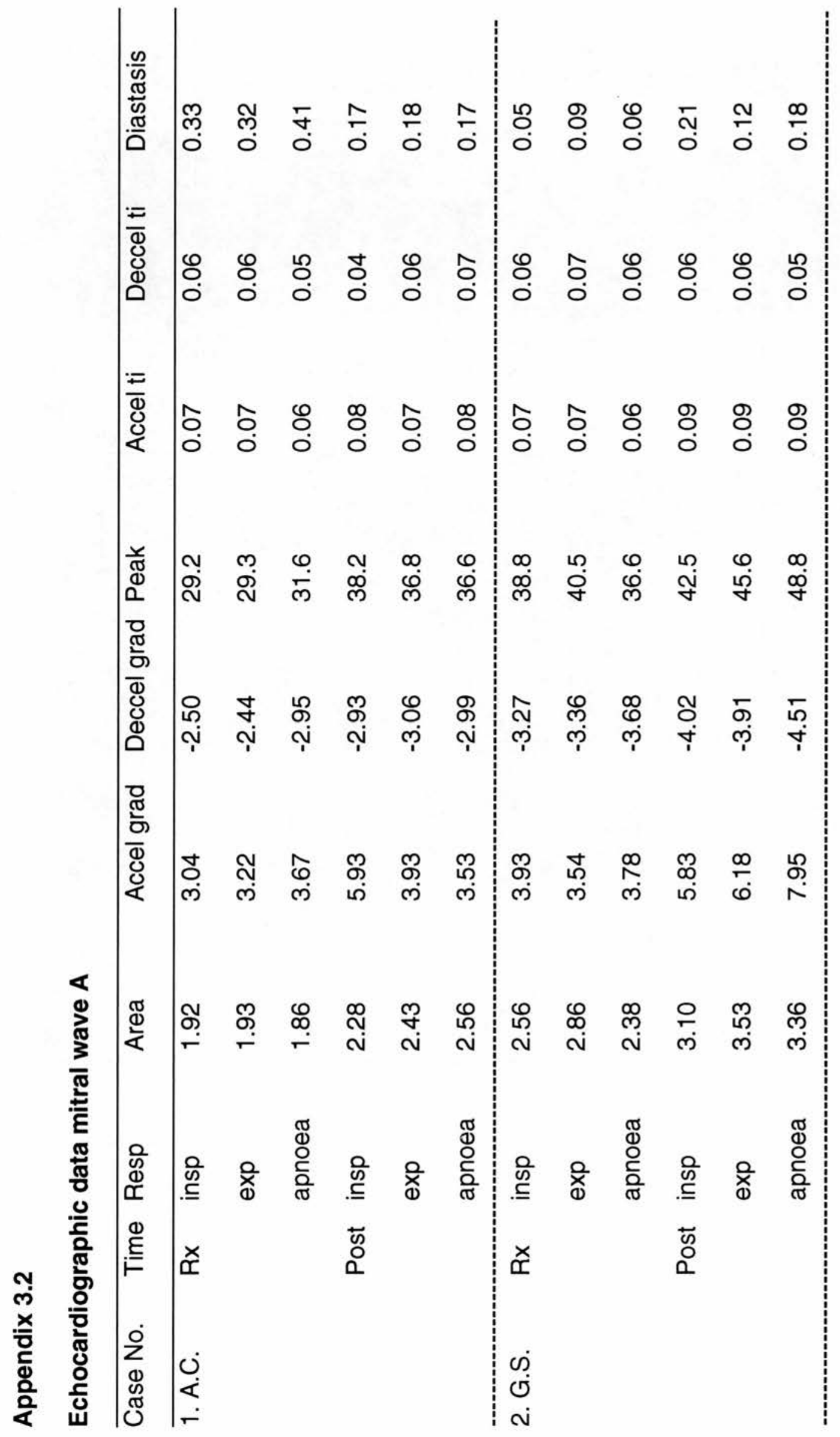




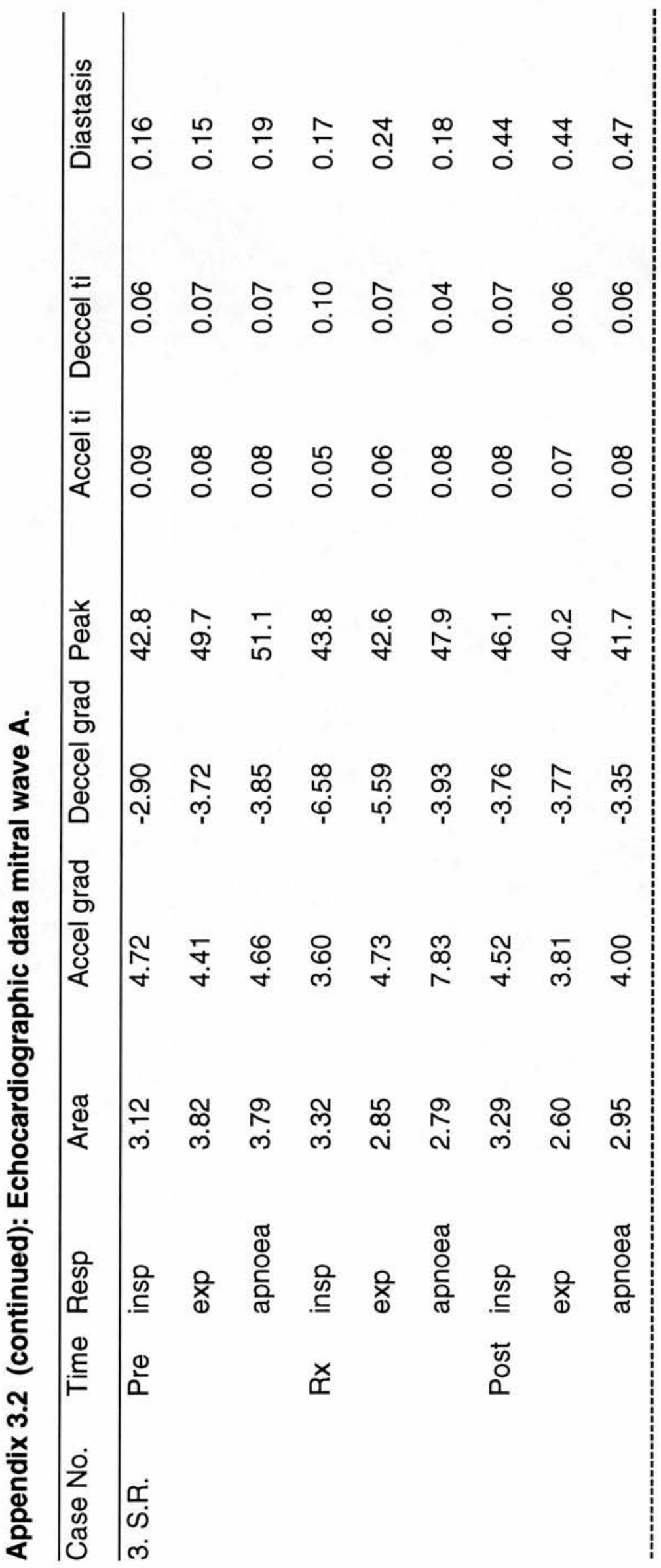









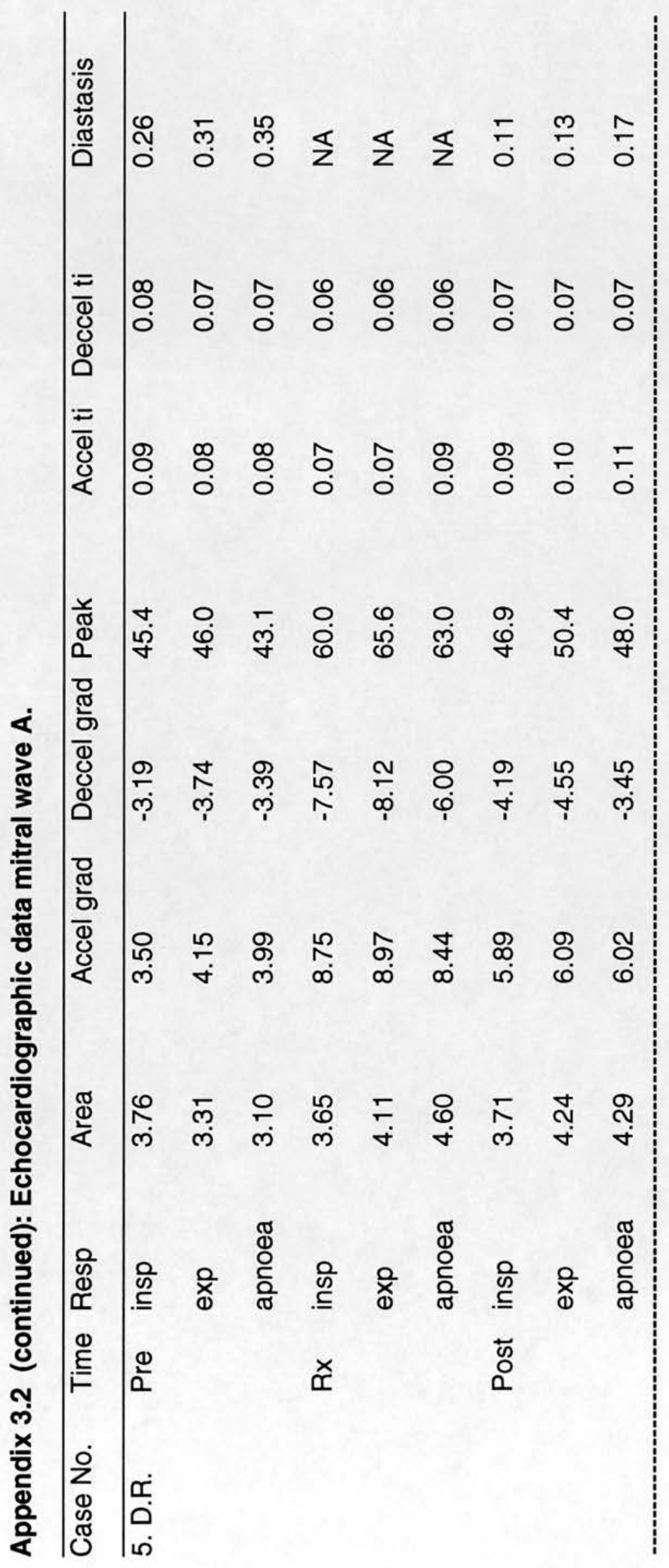




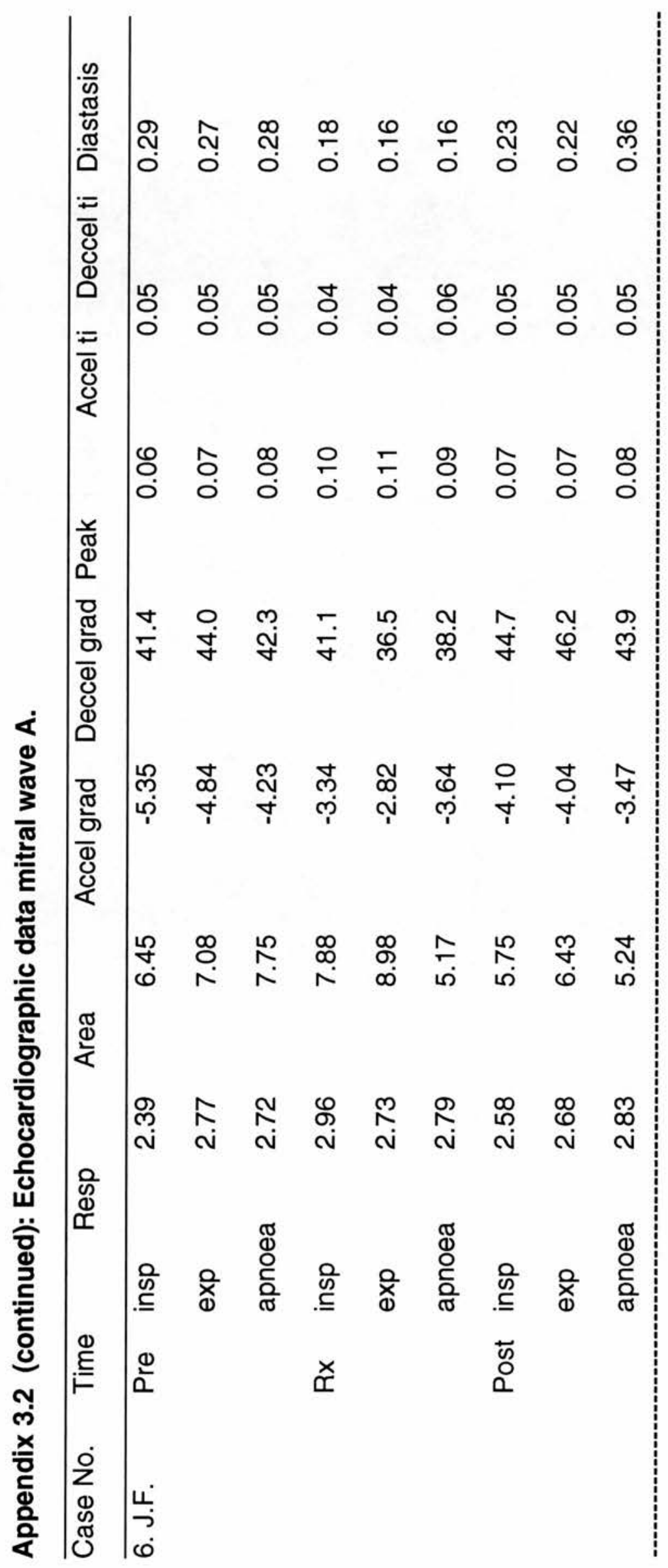




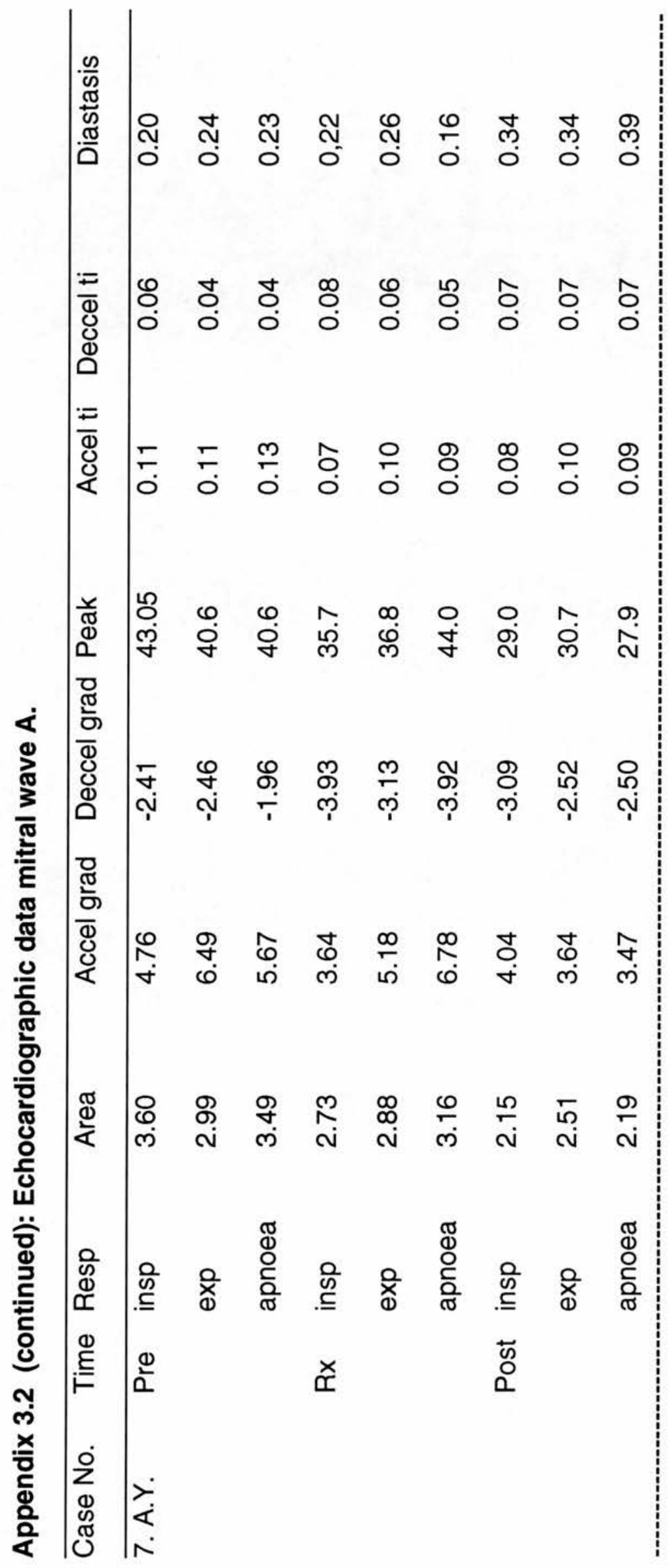




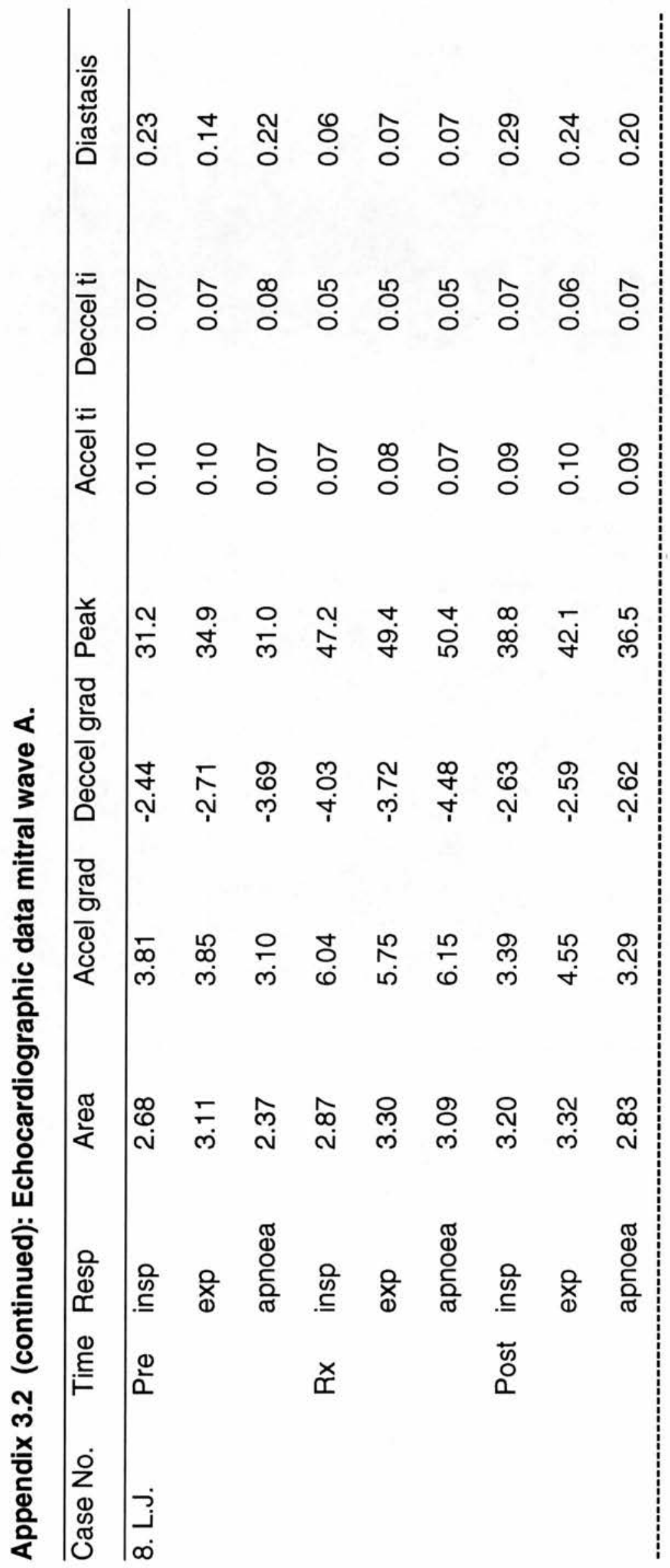




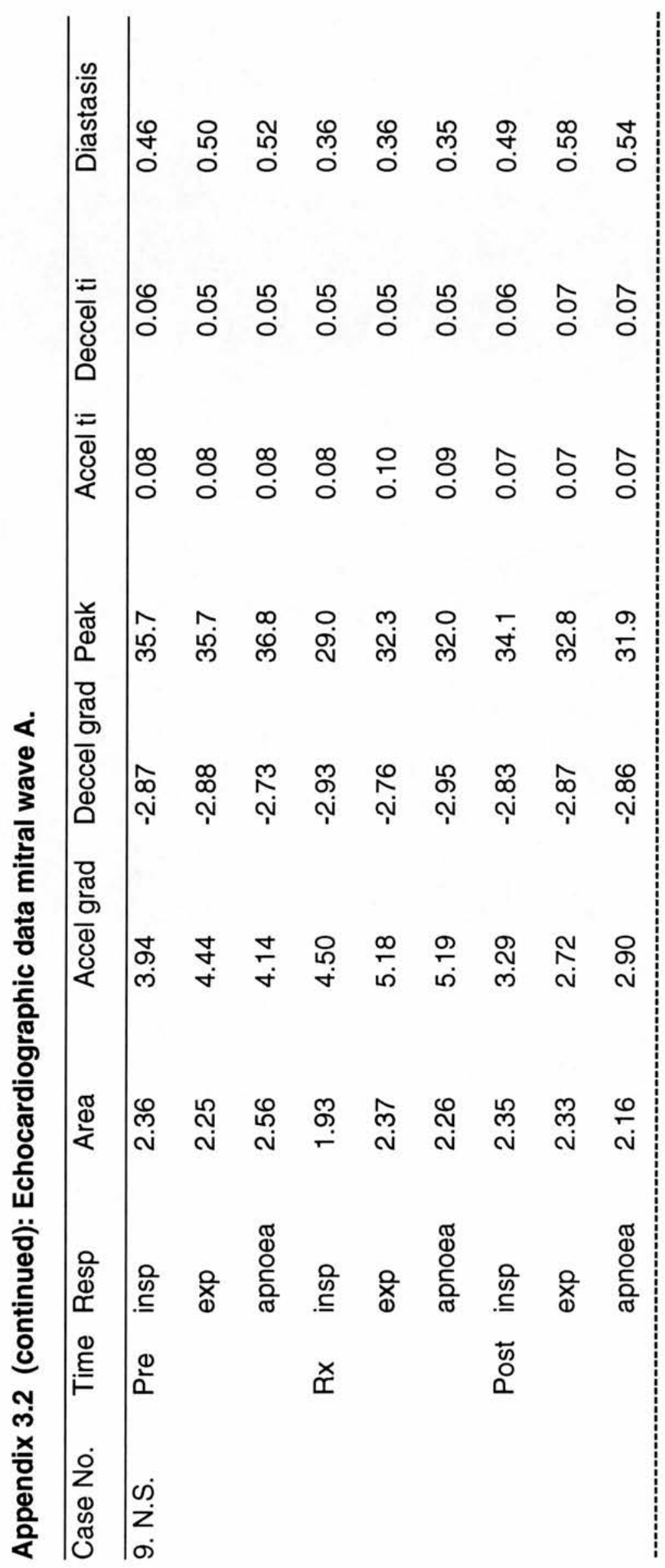




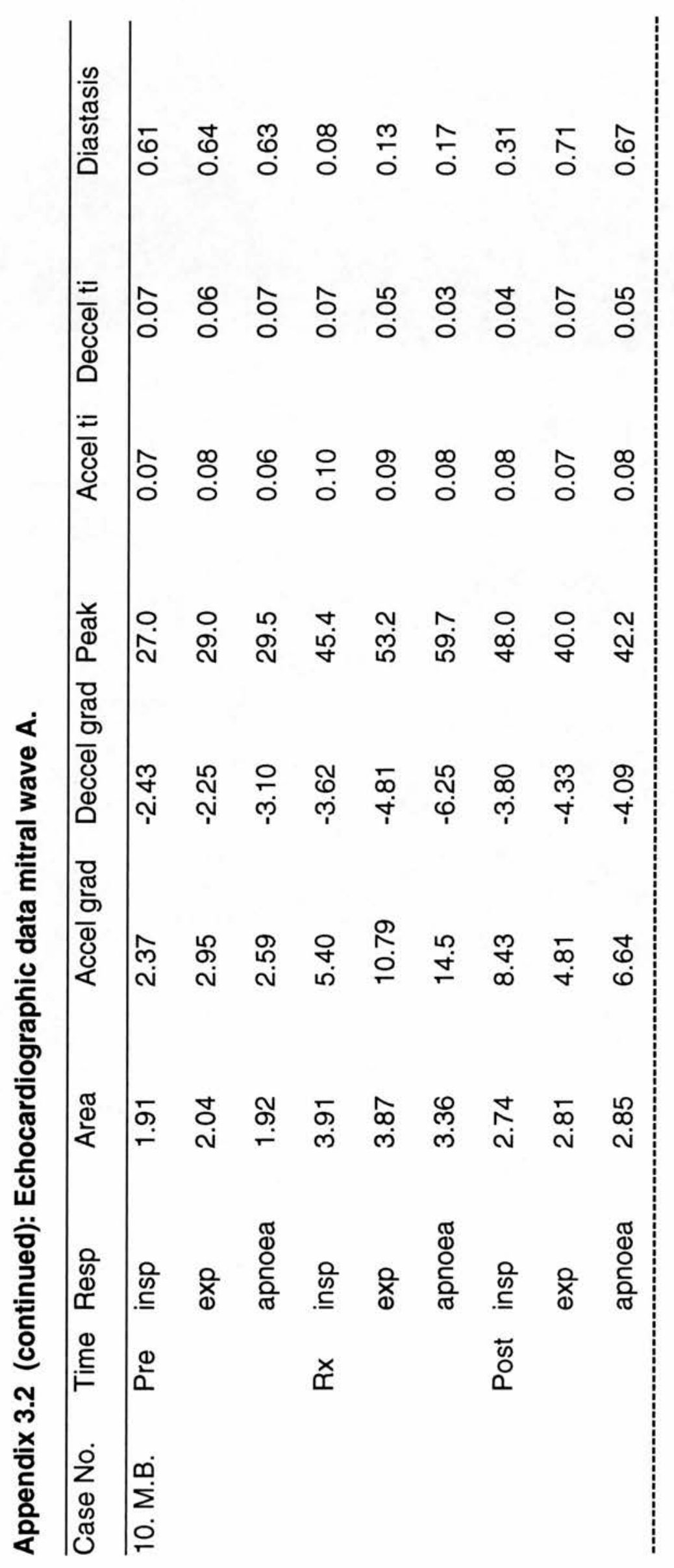




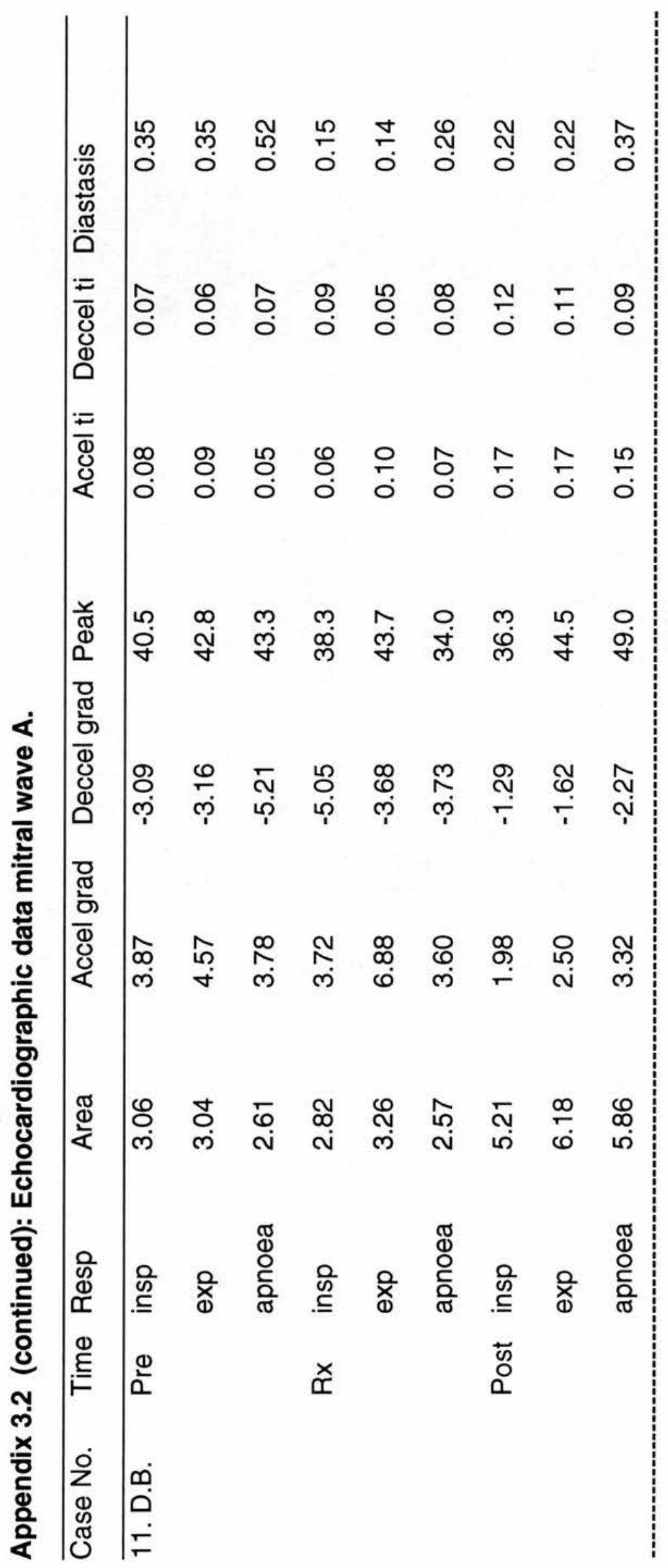




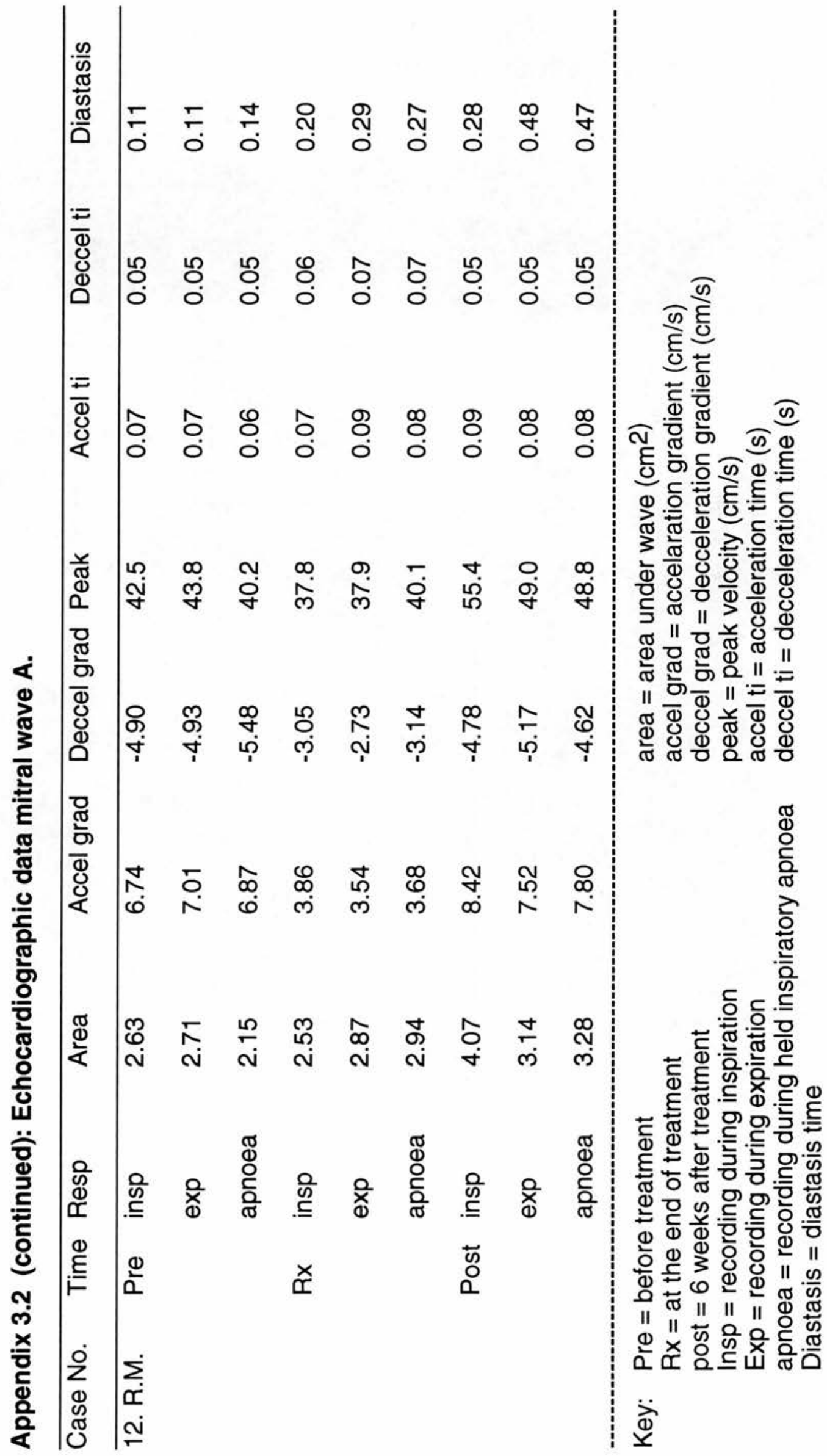


Appendix 3.3

Interventricular relaxation times (IVRT).

\begin{tabular}{|c|c|c|c|c|}
\hline \multirow[b]{2}{*}{ Patient } & \multirow[b]{2}{*}{ Time } & \multicolumn{3}{|c|}{ 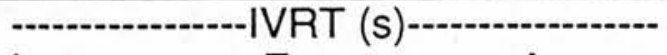 } \\
\hline & & Insp & Exp & Apnoea \\
\hline 1 A.C. & $\begin{array}{l}\text { Rx } \\
\text { Post }\end{array}$ & $\begin{array}{l}0.08 \\
0.07\end{array}$ & $\begin{array}{l}0.07 \\
0.06\end{array}$ & $\begin{array}{l}0.08 \\
0.06\end{array}$ \\
\hline 2 G.S. & $\begin{array}{l}\text { Rx } \\
\text { Post }\end{array}$ & $\begin{array}{l}0.09 \\
0.09\end{array}$ & $\begin{array}{l}0.10 \\
0.10\end{array}$ & $\begin{array}{l}0.09 \\
0.08\end{array}$ \\
\hline 3 S.R. & $\begin{array}{l}\text { Pre-Rx } \\
\text { Rx } \\
\text { Post }\end{array}$ & $\begin{array}{l}0.08 \\
0.05 \\
0.08\end{array}$ & $\begin{array}{l}0.07 \\
0.05 \\
0.09\end{array}$ & $\begin{array}{l}0.09 \\
0.07 \\
0.09\end{array}$ \\
\hline 4 M.L. & $\begin{array}{l}\text { Pre-Rx } \\
\text { Rx } \\
\text { Post }\end{array}$ & $\begin{array}{l}0.09 \\
0.09 \\
0.08\end{array}$ & $\begin{array}{l}0.08 \\
0.08 \\
0.07\end{array}$ & $\begin{array}{l}0.09 \\
0.10 \\
0.08\end{array}$ \\
\hline 5 D.R. & $\begin{array}{l}\text { Pre-Rx } \\
\text { Rx } \\
\text { Post }\end{array}$ & $\begin{array}{l}0.08 \\
0.07 \\
0.07\end{array}$ & $\begin{array}{l}0.07 \\
0.06 \\
0.07\end{array}$ & $\begin{array}{l}0.07 \\
0.06 \\
0.07\end{array}$ \\
\hline 6 J.F. & $\begin{array}{l}\text { Pre-Rx } \\
\text { Rx } \\
\text { Post }\end{array}$ & $\begin{array}{l}0.07 \\
0.08 \\
0.06\end{array}$ & $\begin{array}{l}0.07 \\
0.08 \\
0.06\end{array}$ & $\begin{array}{l}0.07 \\
0.08 \\
0.08\end{array}$ \\
\hline 7 A.Y. & $\begin{array}{l}\text { Pre-Rx } \\
\text { Rx } \\
\text { Post }\end{array}$ & $\begin{array}{l}0.07 \\
0.06 \\
0.06\end{array}$ & $\begin{array}{l}0.07 \\
0.07 \\
0.03\end{array}$ & $\begin{array}{l}0.07 \\
0.07 \\
0.03\end{array}$ \\
\hline 8 L.J. & $\begin{array}{l}\text { Pre-Rx } \\
\text { Rx } \\
\text { Post }\end{array}$ & $\begin{array}{l}0.06 \\
0.05 \\
0.06\end{array}$ & $\begin{array}{l}0.06 \\
0.05 \\
0.09\end{array}$ & $\begin{array}{l}0.07 \\
0.04 \\
0.05\end{array}$ \\
\hline 9 N.S. & $\begin{array}{l}\text { Pre-Rx } \\
\text { Rx } \\
\text { Post }\end{array}$ & $\begin{array}{l}0.06 \\
0.07 \\
0.07\end{array}$ & $\begin{array}{l}0.05 \\
0.06 \\
0.07\end{array}$ & $\begin{array}{l}0.07 \\
0.06 \\
0.06\end{array}$ \\
\hline 10 M.B. & $\begin{array}{l}\text { Pre-Rx } \\
\text { Rx } \\
\text { Post }\end{array}$ & $\begin{array}{l}0.06 \\
0.03 \\
0.05\end{array}$ & $\begin{array}{l}0.06 \\
0.04 \\
0.04\end{array}$ & $\begin{array}{l}0.06 \\
0.03 \\
0.04\end{array}$ \\
\hline
\end{tabular}


Appendix 3.3 (continued):

Interventricular relaxation times (IVRT).

\begin{tabular}{|c|c|c|c|c|}
\hline \multirow[b]{2}{*}{ Patient } & \multirow[b]{2}{*}{ Time } & \multicolumn{3}{|c|}{ 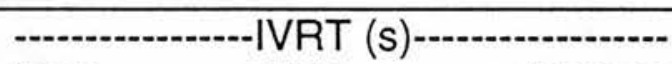 } \\
\hline & & Insp & Exp & Apnoea \\
\hline 11 D.B. & $\begin{array}{l}\text { Pre-Rx } \\
\text { Rx } \\
\text { Post }\end{array}$ & $\begin{array}{l}0.06 \\
0.04 \\
0.06\end{array}$ & $\begin{array}{l}0.07 \\
0.07 \\
0.06\end{array}$ & $\begin{array}{l}0.04 \\
0.08 \\
0.06\end{array}$ \\
\hline 12 R.M. & $\begin{array}{l}\text { Pre-Rx } \\
\text { Rx } \\
\text { Post }\end{array}$ & $\begin{array}{l}0.05 \\
0.07 \\
\text { no pl }\end{array}$ & $\begin{array}{l}0.05 \\
0.05 \\
\text { o ivrt }\end{array}$ & $\begin{array}{l}0.06 \\
0.06\end{array}$ \\
\hline
\end{tabular}

Key: Pre- $\mathrm{Rx}=$ before treatment

$\mathrm{Rx}=$ at end of treatment

Post $=6$ weeks after treatment 


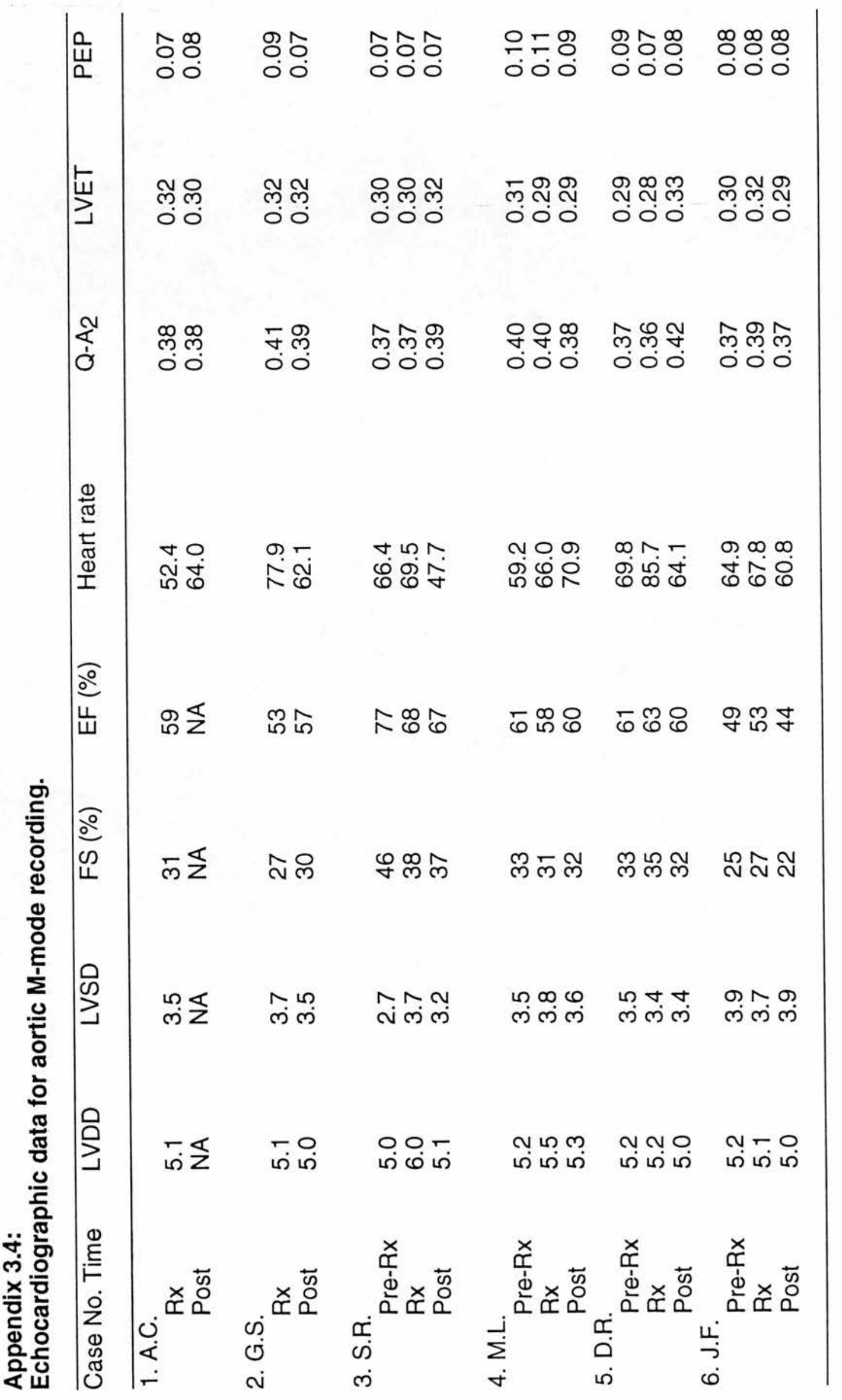




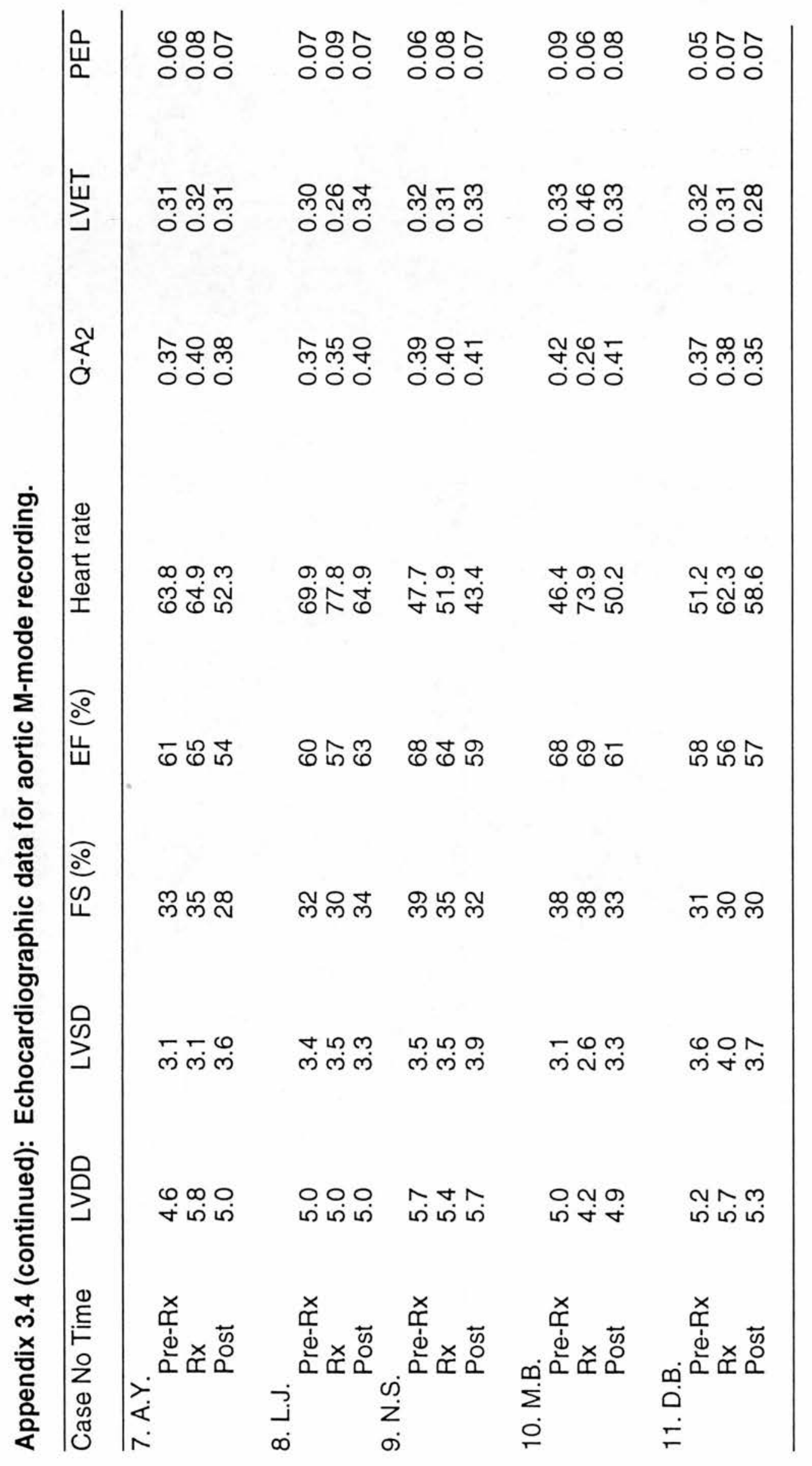




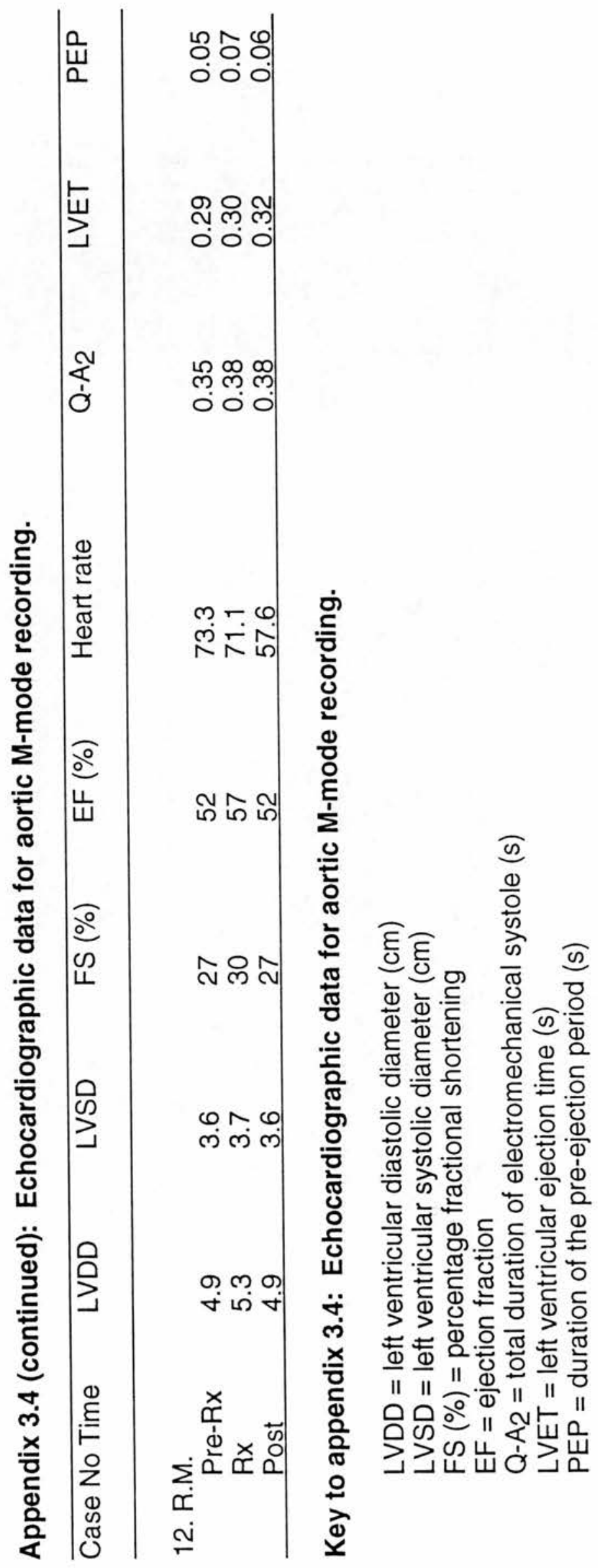




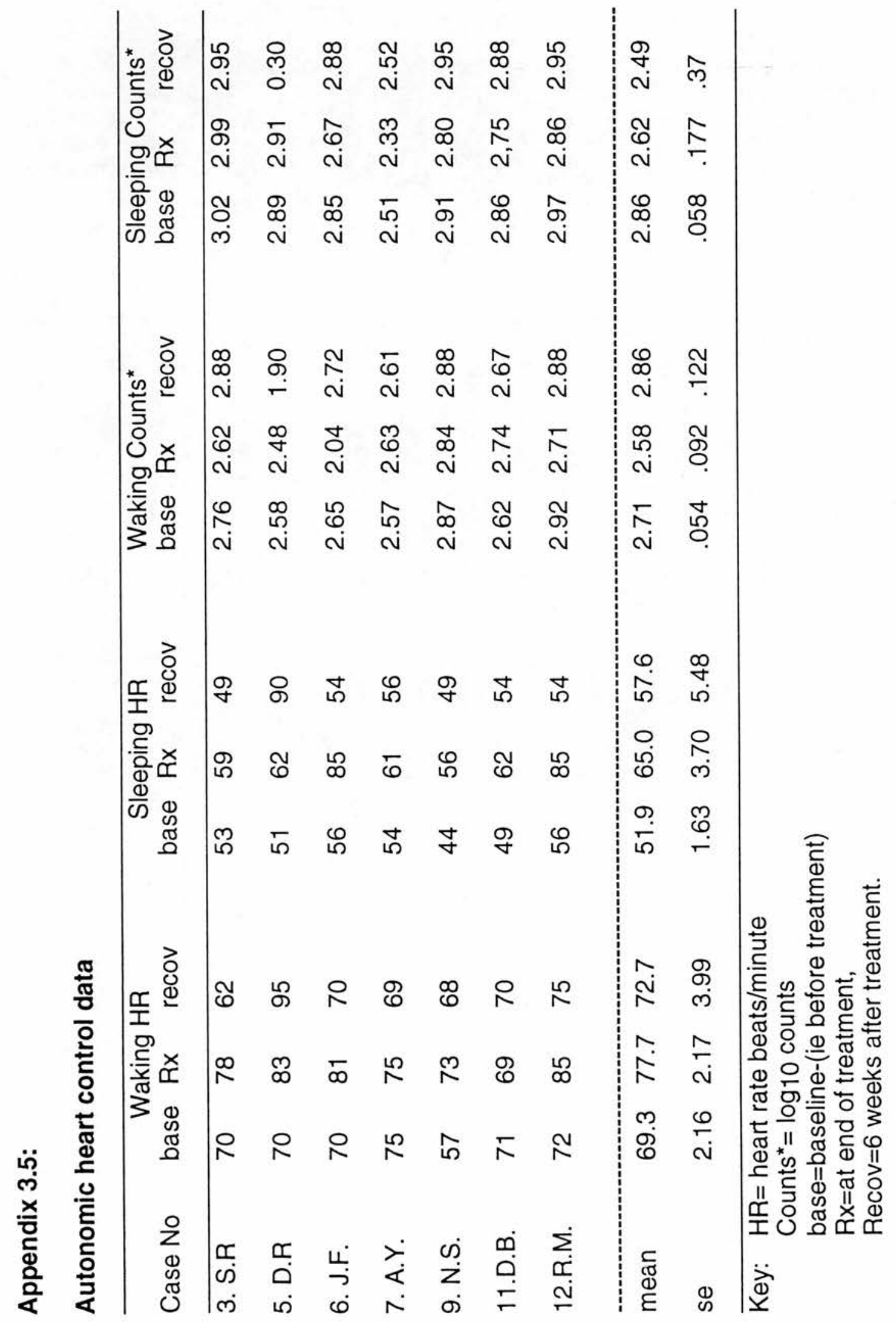




\section{Appendix 3.6}

Electrocardiographic (ECG) data

\begin{tabular}{lclll}
\hline Time & $\begin{array}{c}\text { Axis } \\
\text { mean (se) }\end{array}$ & $\begin{array}{l}\text { R Wave } \\
\text { mean (se) }\end{array}$ & $\begin{array}{l}\text { T Wave } \\
\text { mean (se) }\end{array}$ \\
\hline Day 0 (Pre-Rx) & $59.4(7.15)$ & $21.7(1.55)$ & $18.8(1.94)$ \\
Day 7 & $61.3(5.64)$ & $22.4(1.31)$ & $14.7(2.48)$ \\
Day 14 & $61.7(5.69)$ & 22.6 & $(0.58)$ & $10.4(1.88)$ \\
Day 20 (End-Rx) & $61.7(5.75)$ & $21.2(1.25)$ & $7.4(1.98)$ \\
Day 34 & $60.6(6.71)$ & $20.7(1.95)$ & $15.9(0.69)$ \\
Day 64 & $58.0(6.16)$ & $20.0(1.07)$ & $18.5(2.92)$
\end{tabular}

The values shown are:

Heights of $R$ waves measured in $\mathrm{mm}$

Sum of heights of $T$ waves in $V_{4} V_{5} \& V_{6}$ in $\mathrm{mm}$. 
Appendix 4.1

\section{Patient Informed Consent}

An open prospective trial comparing systemic sodium stibogluconate (Pentostam) with systemic aminosidine (paromomycin) in the treatment of American Cutaneous Leishmaniasis.

You are being asked to participate in a study to investigate whether a new treatment for cutaneous leishmaniasis is as effective as the standard treatment used in the British army. The standard treatment consists of daily injections of Pentostam for 20 days which cures nearly all ulcers, however, many patients are unwell whilst having this treatment. The new treatment, consisting also of daily injections for 20 days, has been reported to be safe and to cause few problems. Although it is known to work in other forms of leishmaniasis we do not yet know if it will work in patients with the form that causes skin ulcers in Belize.

Before any treatment is started background information will be taken relating to your disease and a sample (under local anaesthetic) will be taken to identify the organism causing the infection. Your progress will be carefully assessed by the doctor throughout the treatment and blood tests performed to check for any ill-effects.

Your participation in this study is requested because the army medical services are keen to develop a more acceptable cure for your illness, however, it must be proven to work as well as the standard treatment.

If you enter the trial you will be given either the standard or the new treatment determined at random. You are free to refuse to participate, either now or later in during the study, and this will not affect the relationship between you and your doctor, nor will it deprive you of the possibility of receiving other treatments for your infection.

All information gained about you and your condition will be treated in the strictest confidence.

If we find out information about these drugs which might affect your decision whether or not to take part in the study we will make sure you are informed. The doctor may stop your participation in the study at any time for safety reasons.

\section{Patient Written Consent}

I................................have read and understood the information sheet for this study and agree to participate.

Signed

Date
Signed.

Date. 


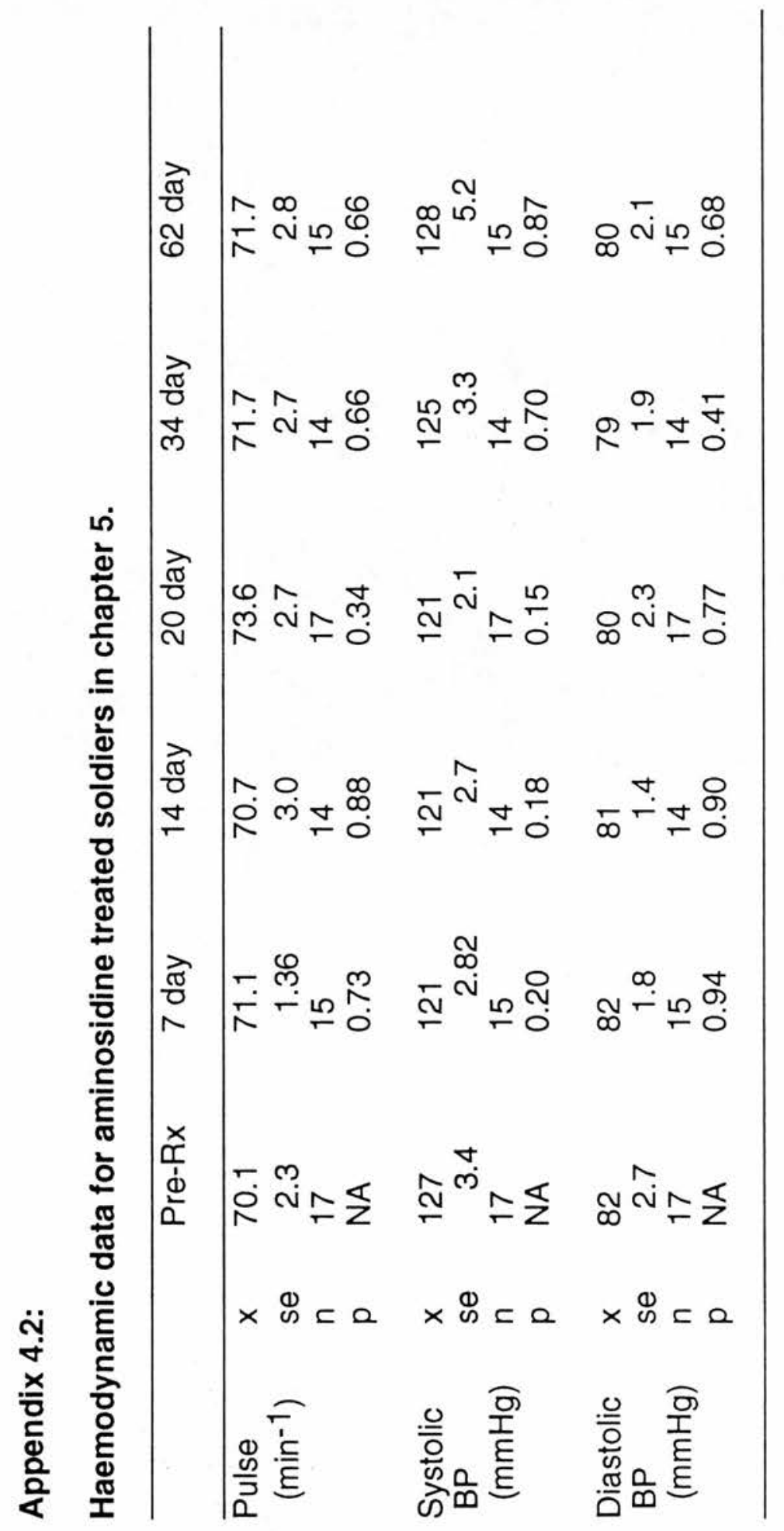




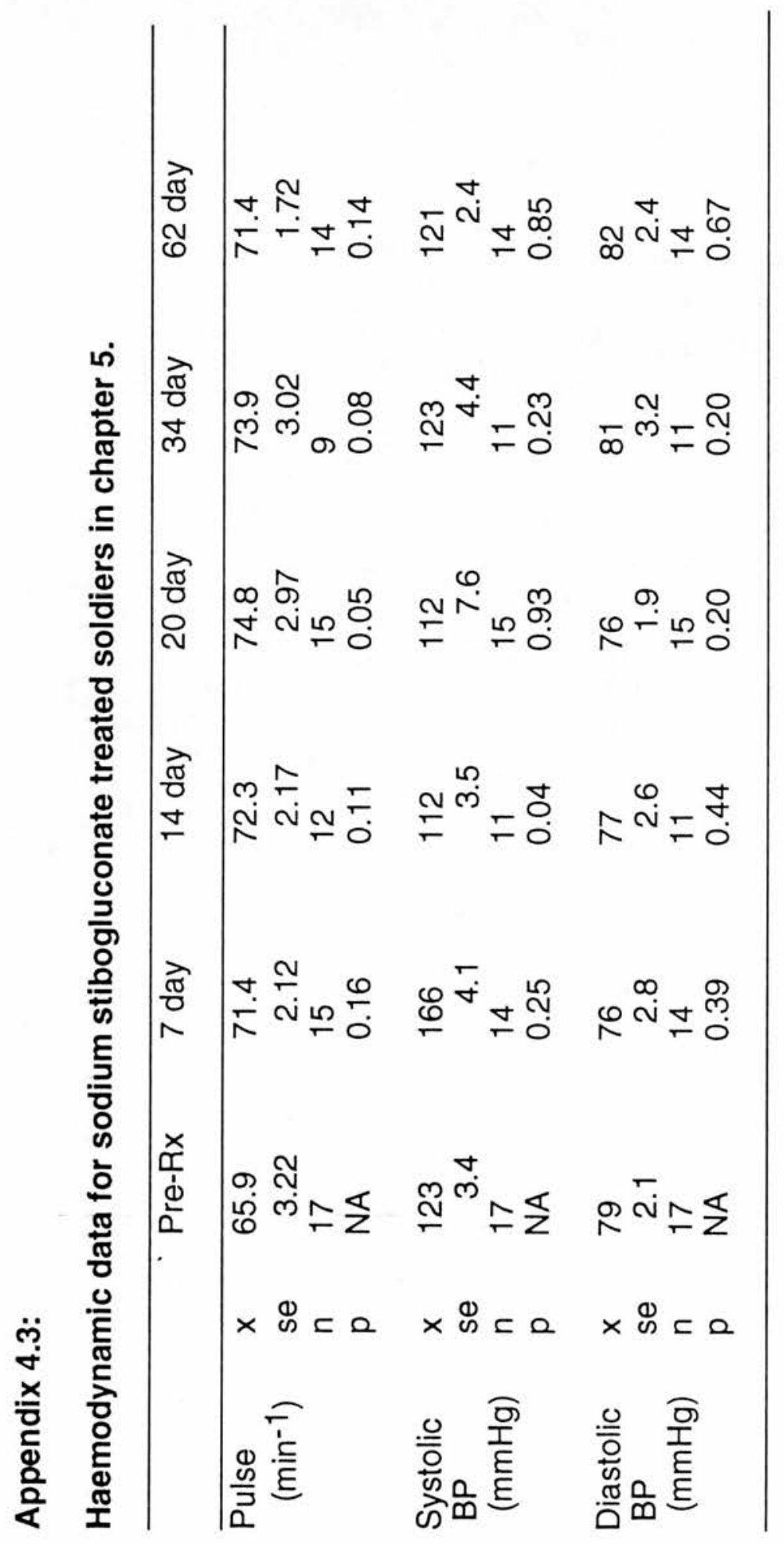




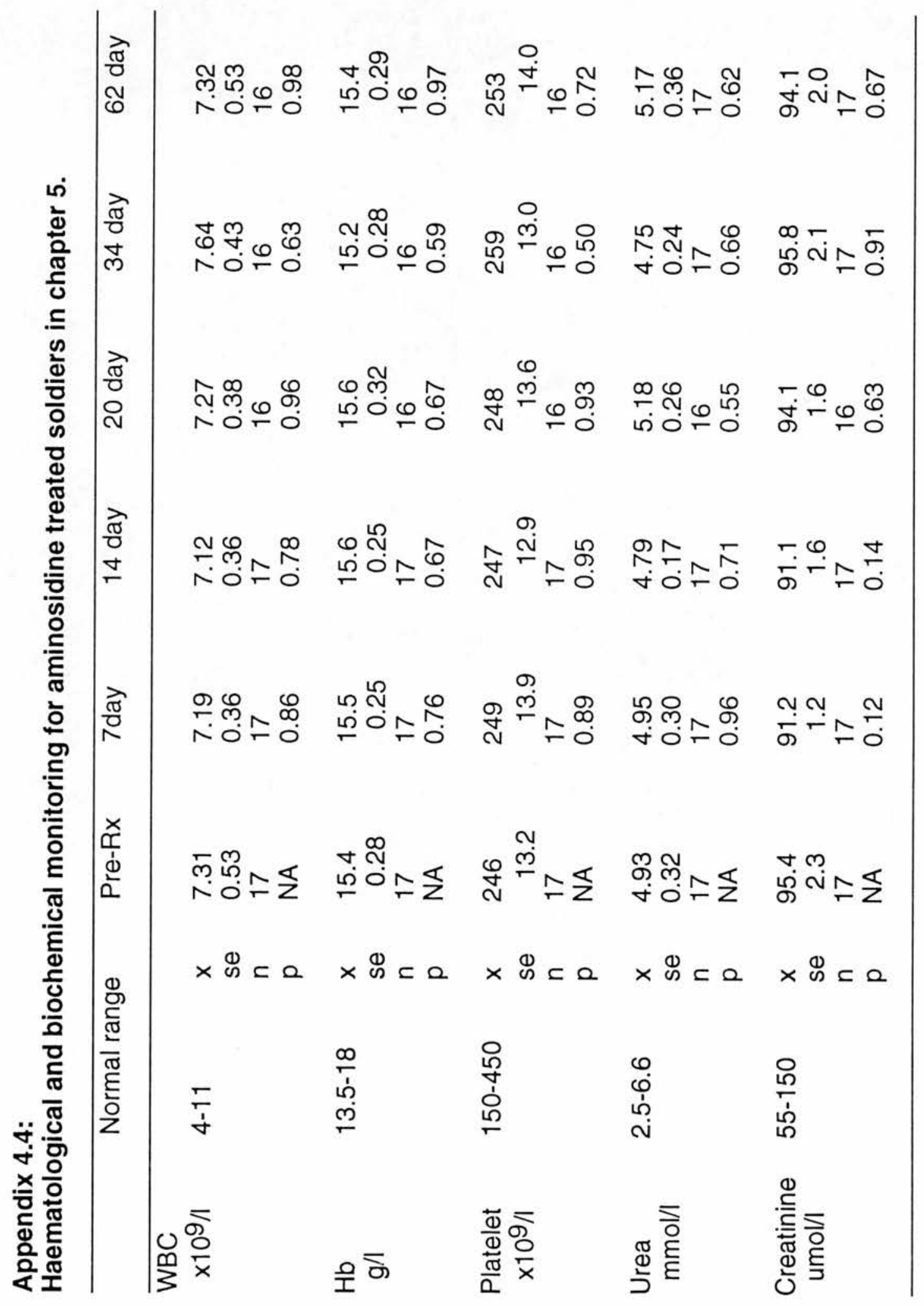




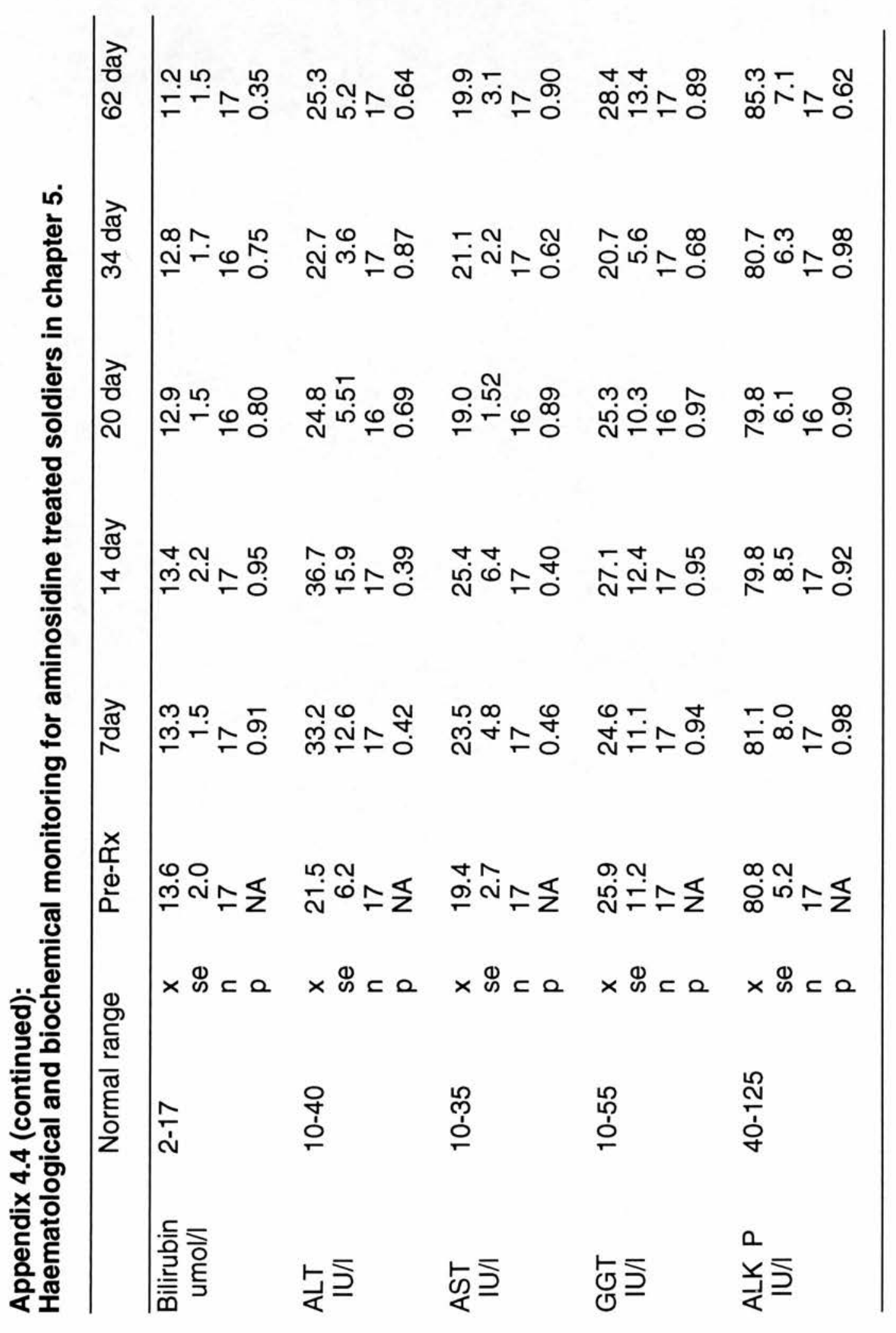


216

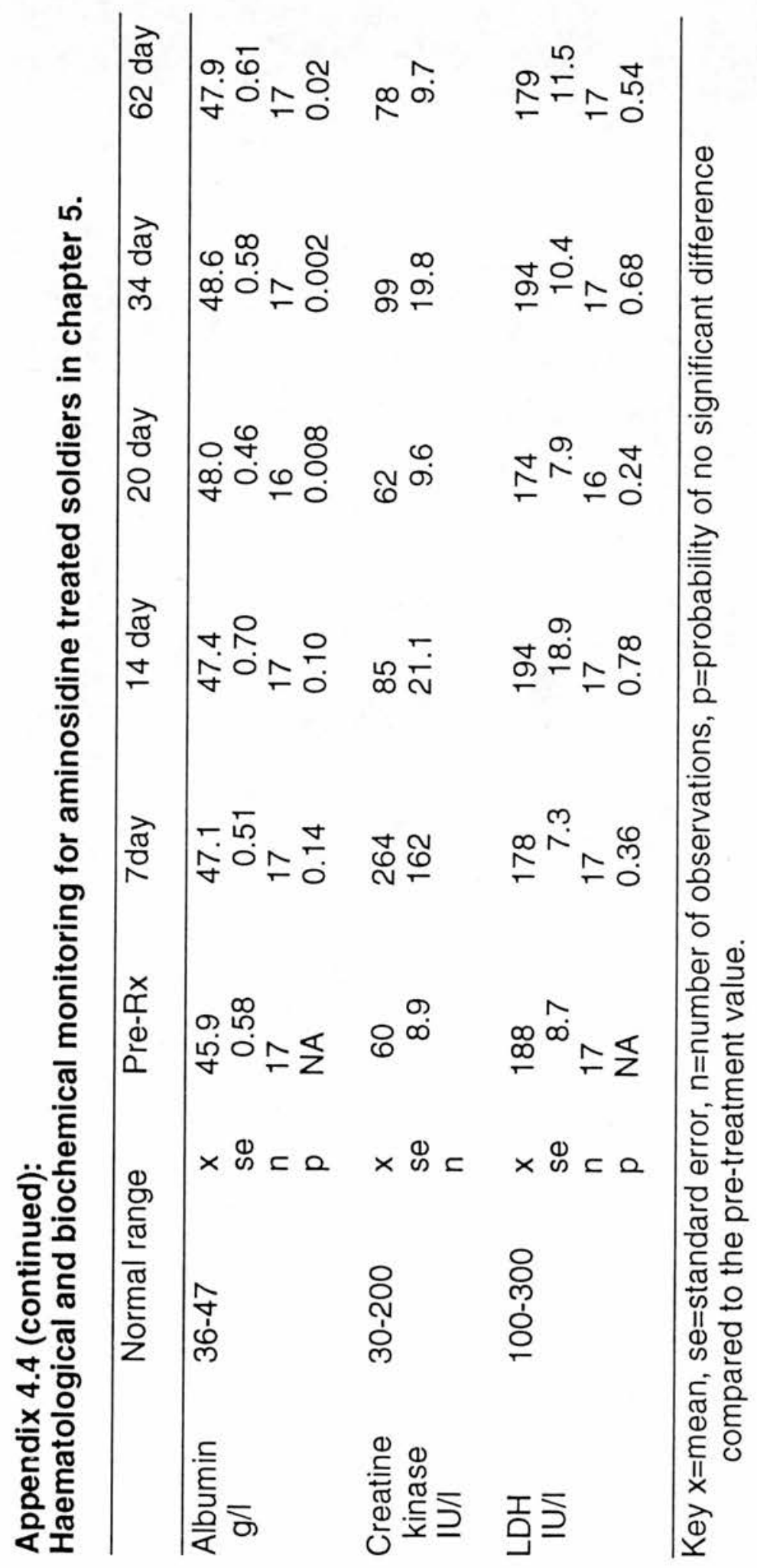




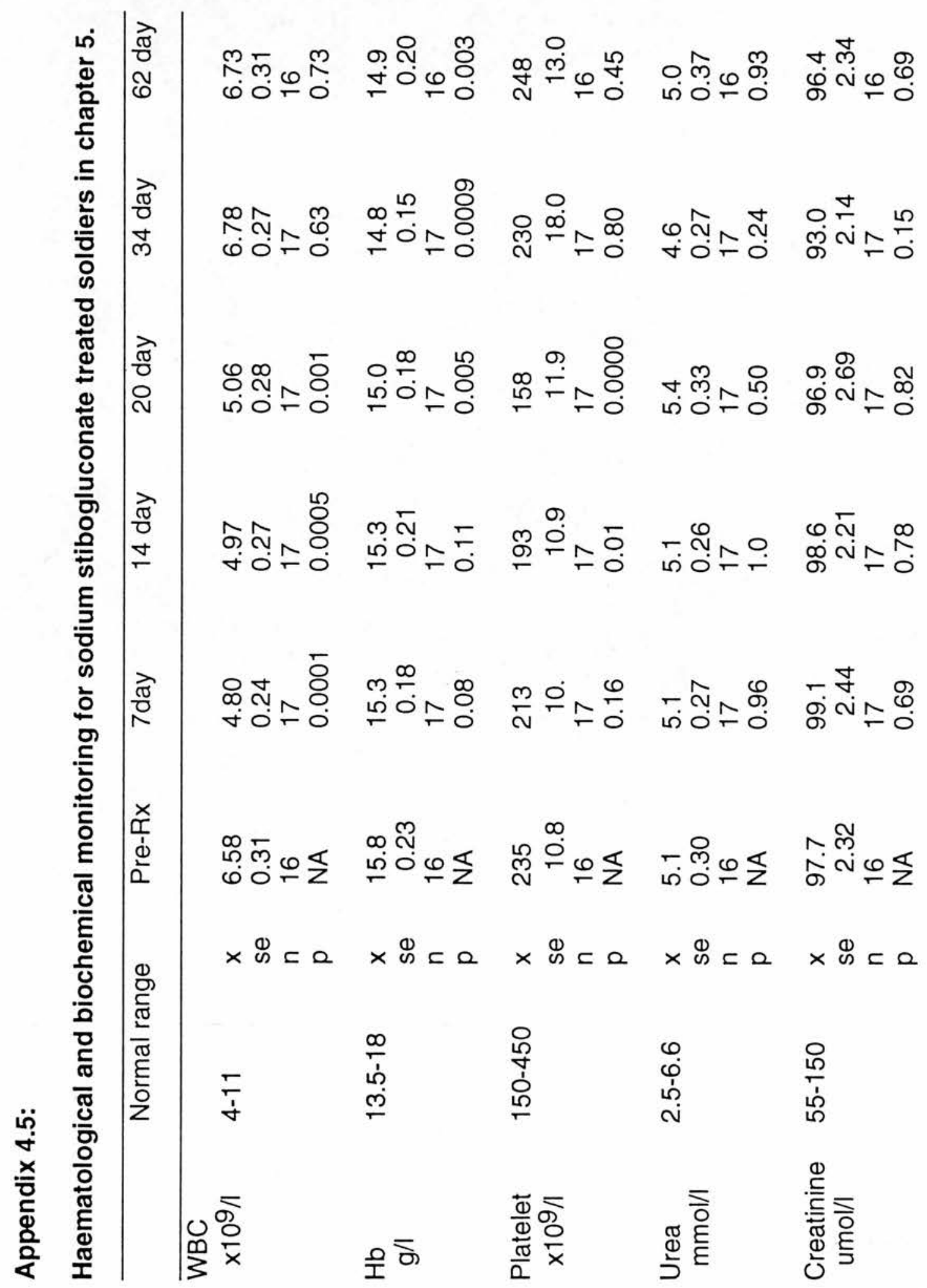




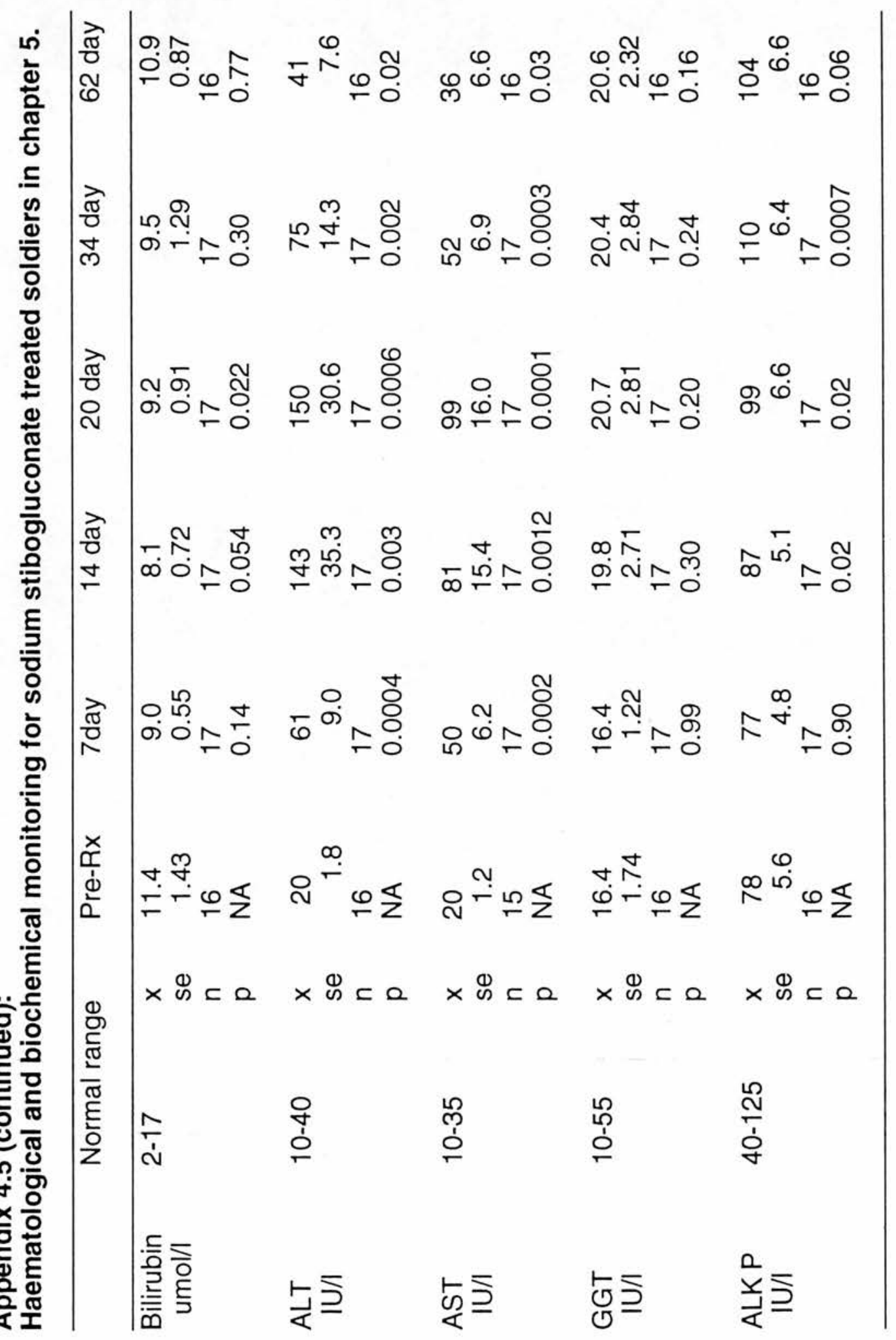




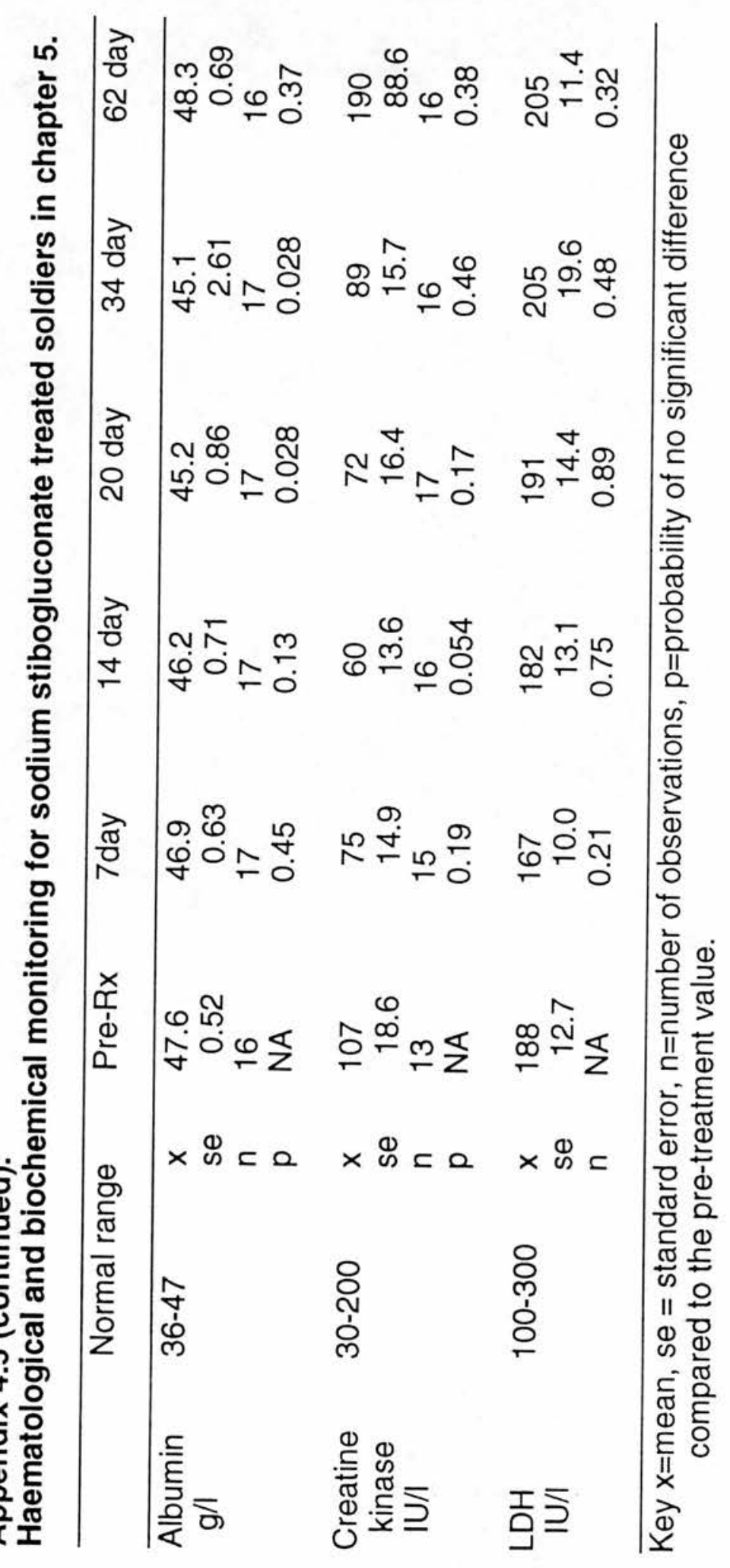




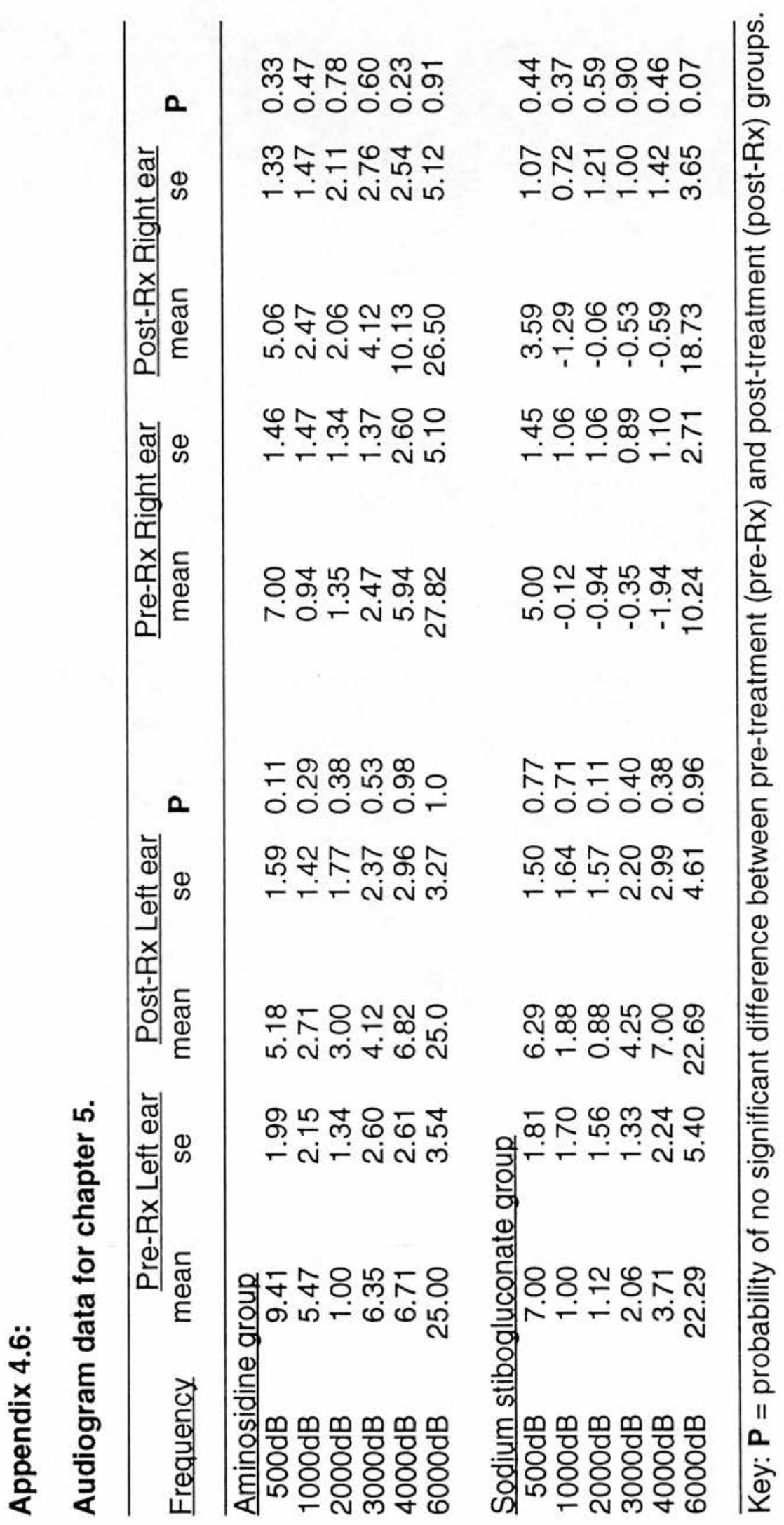




\title{
Cutaneous leishmaniasis in British troops from Belize
}

\author{
N.C.HEPBURN, M.J.TIDMAN AND J.A.A.HUNTER \\ University Department of Dermatology. The Royal Infirmary. Edinburgh EH3 9YW. U.K. \\ Accepted for publication 7 August 1992
}

Summary The medical records of 306 British soldiers in whom a clinical diagnosis of cutaneous leishmaniasis had been made following a tour of duty in Belize were analysed. Parasitological confirmation of the diagnosis was established in 187 cases; leishmania were cultured in 117 cases and LeishmanDonovan bodies were identified histologically in a further $7 \mathrm{C}$ cases. Leishmania braziliensis braziliensis was identified in 78 cases and Leishmania mexicana mexicana in a further 29 cases. Seventy-one per cent of patients had a single lesion which, in most cases, occurred on the exposed extremities. The mean diameter of the ulcers was $14.4 \mathrm{~mm}$. Treatment with sodium stibogluconate was effective. Two regimens were used, consisting of either $600-800 \mathrm{mg}$ daily given initially for 10 days, or $600 \mathrm{mg}$ b.d. given initially for 14 days. Of those allocated to the lower dose regimen $48.5 \%$ were cured after the initial 10-day course, and ultimately the ulcers of $93 \%$ of patients healed following more prolonged treatment at this dose. Of those allocated to the higher dose regimen $63.9 \%$ were cured after the initial 14-day course and ultimately the ulcers of all patients healed after more prolonged treatment at this dose. A transient leucopenia and a rise in liver enzymes were noted during treatment, and these changes were dose-dependent. No cases of mucocutaneous leishmaniasis were encountered.

American cutaneous leishmaniasis (CL) is a major health problem in Central and South America, and is increasing in incidence. ${ }^{1}$ It is being encountered more frequently in temperate zones of the western world as international tourism to endemic areas increases. Although leishmaniasis has been recognized since antiquity, the epidemiology, clinical features and treatment are poorly described in the literature. In 1978 the British Army established a permanent garrison of approximately 1500 troops in Belize, since when over 300 servicemen have been treated for CL. Initially, all cases of Belizian CL were assumed to be caused by Leishmania mexicana mexicana (Lmm) which causes a cutaneous ulcer, which eventually heals spontaneously. However, Leishmania braziliensis braziliensis (Lbb) was subsequently identified in many of the cases. ${ }^{2}$ Although CL due to Lbb also presents as a cutaneous ulcer, the destructive mucocutaneous form of the disease ('Espundia') may subsequently develop due to metastatic spread. The clinical features and treatment of 187 cases of Belizian CL, in which a parasitologically proven diagnosis was made, are described.

\section{Methods}

\section{Patients and methods}

All servicemen in whom CL was suspected were referred to the Dermatology Department of the Cambridge Military Hospital in Aldershot for clinical assessment, investigation and treatment. A clinical diagnosis of CL had been made in 306 servicemen between 1978 and mid1990. following a tour of duty in Belize. Their hospital case notes were scrutinized and the following data extracted: age; dates of Belize tour; date that the first lesion was noticed; number, size and site of the lesions; the results of histological examination of the lesion; culture and isoenzyme typing ${ }^{2}$ of the parasite. Detailed records were made of the treatment given including dates, drug, dose, duration, side-effects, and the haematological and biochemical parameters used in monitoring treatment. Resolution of the ulcers, an objective endpoint which could be assessed easily and reliably by all physicians, was the standard criterion for cure. Patients were closely supervised by Army dermatologists to ensure that cure was attained and maintained for at least 3 months. Thereafter, patients were usually followed-up by their regimental medical officer, and rereferred if relapse occurred.

For the purposes of this study, analysis was limited to 


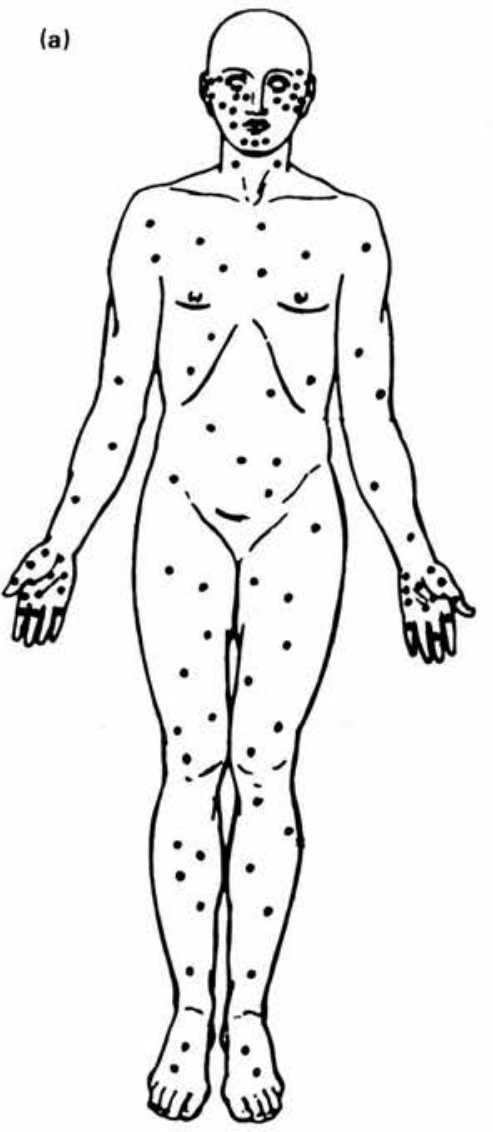

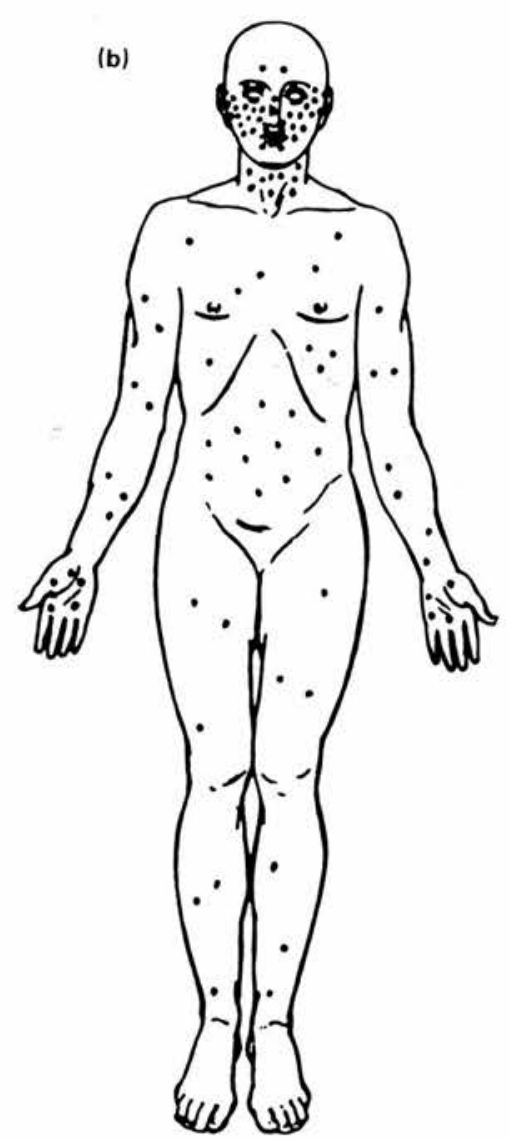

Figure 1. (a) Sites of lesions in cases due to $\mathrm{Lbb}(-1 \%$ of lesions; total 114 lesions in 77 cases). (b) Sites of lesions in cases due to Lmm $(\bullet=1 \%$ of lesions; total 43 lesions in 29 cases). the 187 cases in which the clinical diagnosis was confirmed by demonstration of the parasite by culture and/or histological identification of Leishman-Donovan (LD) bodies. A comparison of the clinical features was made between those cases in which Lbb was identified and those in which Lmm was identified. During the early part of the 12-year period covered by this study the main treatment strategy consisted of an initial 10-day course of intravenous sodium stibogluconate (Pentostam ${ }^{\circledR}$ ) 600-800 mg daily. If the ulcers were not healing satisfactorily this was either continued or repeated after a break, as recommended by the manufacturer. ${ }^{3}$ Because patients required repeated courses a higher dose regimen was subsequently adopted. This consisted of an initial 14-day course of intravenous sodium stibogluconate $600 \mathrm{mg}$ twice daily. Again, if the ulcers were not healing satisfactorily, this was either continued or repeated after a break. The success of these strategies was compared. Haematological and biochemical parameters prior to treatment, at the end of treatment, and 2 weeks after cessation of treatment, were available for 2 cohorts: one treated with $600 \mathrm{mg}$ i.v. daily to a total of 6 $\mathrm{g}$ ( 36 cases) and a second treated with $600 \mathrm{mg}$ i.v. twice daily to a total of $16.8 \mathrm{~g}$ ( 24 cases). Cases were followed up for at least 3 months after clinical cure.

\section{Statistical analysis}

Pretreatment lesion size and duration in the total patient group, the Lmm and Lbb subgroups, and between the two treatment strategies, were statistically evaluated using an unpaired $t$-test. Differences in haematological and biochemical parameters before, during and after treatment, were evaluated using a paired $t$-test.

\section{Results}

A clinical diagnosis of CL was made in 306 otherwise healthy male soldiers, mean age 23 years, who had acquired their infection whilst on active service in Belize. Confirmation of the diagnosis by demonstration of the parasite was made in 187 cases $(61 \%)$ : a positive culture was obtained in 117 cases, and LD bodies were identified in a further 70 cases. Of the 117 culture-positive cases the species was identified in 107: 77 were due to Lbb, 29 to Lmm, and in one case both Lbb and Lmm were identified from a single lesion. Lesions started as small inflammatory papules which gradually enlarged and then ulcerated. The ulcer was often covered by adherent crust which subsequently separated to exp a necrotic ulcer base which was frequently second? 
Table 1. Features of the low- and high-dose regimen cohorts

\begin{tabular}{lcc}
\hline & Low-dose regimen & High-dose regimen \\
\hline Number of patients & 101 & 72 \\
Mean number of lesions & $1 \cdot 54$ & $1 \cdot 47$ \\
Mean duration of lesions prior to treatment (weeks) & $9 \cdot 16$ (SD 6.9) & $8 \cdot 84($ SD $4 \cdot 43)$ \\
Mean lesion size (longest axis of ulcer, mm) & $15 \cdot 89^{*}($ SD $9 \cdot 94)$ & $12 \cdot 3^{*}($ SD $7 \cdot 74)$ \\
& & \\
Lesion site & $39(25) \dagger$ & $28(26)$ \\
Head and neck & $26(17)$ & $16(15)$ \\
Trunk & $16(10)$ & $14(13)$ \\
Arms & $42(27)$ & $25(24)$ \\
Hands/wrists & $31(20)$ & $20(19)$ \\
Legs & $2(1)$ & $3(3)$ \\
Not recorded & & \\
Species typing & 18 & 5 \\
Lmm & 41 & 1 \\
Lbb & 0 & 35 \\
Lmm and Lbb & 42 & \\
Not available & & \\
\hline
\end{tabular}

* Significant difference $P<0 \cdot 05$.

$\dagger$ Numbers in parentheses are percentages. infected with either Staphylococcus aureus or $\beta$-haemolytic streptococci. The fully developed lesion consisted of a painless ulcer with a necrotic base and an indurated violaceous margin. A total of 283 lesions were identified in the 187 parasitologically confirmed cases: a solitary lesion was present in $71 \%$, two lesions in $16 \%$, three lesions in $8 \%$ and four lesions in $4 \%$. One soldier had five lesions and another had 12 lesions. Cases due to Lmm and Lbb had similar numbers of lesions, and most occurred on exposed body sites, with the head, neck, wrists and hands accounting for $51 \%$ of lesions. Lesions due to Lmm were more common on the head and neck than those due to Lbb (Fig. 1). The mean diameter (longest axis) of all ulcers was $14.4 \mathrm{~mm}$ (SD 9.3), lesions on the head and neck being smaller than those elsewhere, at presentation. The lesions due to Lbb were significantly $(P<0 \cdot 01)$ larger (mean diameter $17 \mathrm{~mm})$ than those due to Lmm (mean diameter $12.3 \mathrm{~mm}$ ), despite the observation that Lbb lesions had been present for a significantly $(P<0.05)$ shorter time (mean 8.8 weeks) than Lmm lesions (mean 12.5 weeks). Most patients presented either during their Belize tour of duty, or within 4 months of their return. One patient presented 11 months after his return.

Treatment started after a mean interval of 9.9 weeks (SD 6.7) had elapsed after the first lesion was noticed. Sodium stibogluconate was the mainstay of treatment. The ulcers of 166 soldiers healed with sodium stiboglupnate alone, although there was a wide range in the dose required (mean $13 \cdot 1 \mathrm{~g}$, SD $7 \cdot 2$ ). During the early part of the study 101 patients were treated with a low (600-800 mg) daily dose regimen. In only one case were clinical side-effects recorded - the patient reported aching muscles after receiving $800 \mathrm{mg}$ daily for 11 days. The higher dose regimen (600 mg twice daily) which was subsequently adopted was associated with greater morbidity; of the 72 patients who received this regimen three became nauseated and anorexic, 11 developed myalgia, two developed chest pain (which was associated with tender costal cartilages), two reported nonspecific malaise and two developed thrombophlebitis at the site of the indwelling i.v. cannula, associated with pyrexia. One patient developed cheiropompholyx, although this resolved with appropriate treatment, despite continuing sodium stibogluconate. One patient developed an acute aseptic arthritis of his knee 2 days after completing a course of $16.8 \mathrm{~g}$ stibogluconate, and another developed severe hip pain, accompanied by a creatine kinase of $25,290 \mathrm{IU} / \mathrm{l}, 14$ days after receiving $23.4 \mathrm{~g}$ sodium stibogluconate. Both problems gradually resolved over the following 4 months without specific therapy.

Sequential haematological and biochemical monitoring parameters, assessed prior to treatment, at the end of treatment, and 2 weeks after treatment was stopped, were available for two cohorts. Although laboratory data was available for other patients, it was not collected systematically and, therefore, its analysis could have been misleading. The first cohort received the standard low-dose regimen consisting of $600 \mathrm{mg} /$ day to a total of 
Table 2. Haematological and biochemical changes during treatment with sodium stibogluconate

\begin{tabular}{|c|c|c|c|c|c|c|}
\hline & Pretreatment & Post-treatment & Convalescent & Pretreatment & Post-treatment & Convalescent \\
\hline White cell count & $\mathrm{N}$ & $\downarrow$ & $\mathrm{N}$ & $\mathrm{N}$ & $\downarrow$ & $\mathrm{N}$ \\
\hline Alkaline phosphatase & $\mathrm{N}$ & $\mathrm{N}$ & $\uparrow$ & $\mathrm{N}$ & $\uparrow$ & $\uparrow$ \\
\hline Gamma glutamyl transferase & $\mathrm{N}$ & $\mathrm{N}$ & $\mathrm{N}$ & $\mathrm{N}$ & $\uparrow$ & $\uparrow$ \\
\hline Creatine kinase & $\mathrm{N}$ & $\downarrow$ & $\mathrm{N}$ & $\mathrm{N}$ & $\downarrow$ & $\mathrm{N}$ \\
\hline
\end{tabular}

Changes are indicated at the end of treatment and 2 weeks after treatment was stopped.

$\mathrm{N}$, within normal range, arrows indicate significant variation $(P<0.05)$ from the pretreatment value.

$6 \mathrm{~g} \mathrm{(36}$ patients) and the other received the standard high-dose regimen consisting of $600 \mathrm{mg}$ twice daily to a total of $16.8 \mathrm{~g}$ ( 24 patients). The characteristics of these two groups are compared in Table 1. A mild, although significant $(P<0 \cdot 05)$, leucopenia was noted at the end of treatment in both groups, but there was no change in the haemoglobin level. The serum urea did not rise during treatment. Serum transaminase levels (alanine aminotransferase and aspartate aminotransferase) were both elevated at the end of treatment, but had almost returned to their pretreatment levels 2 weeks later. This change was much more marked in the group which received the high-dose regimen. The serum glutamyl transferase was only elevated in the high-dose group at the end of treatment, and had also almost returned to normal 2 weeks later. The alkaline phosphatase level was elevated in the high-dose group at the end of treatment, and in both groups 2 weeks later. A transient fall in serum creatine kinase at the end of treatment was noted in both groups. There was no significant elevation in the level of lactate dehydrogenase. These changes are summarized in Table 2.

The low daily dose sodium stibogluconate regimen (600-800 mg daily) was initiated in 101 soldiers. Treatment was given for 10 days in the first instance, after which it was either continued, or repeated after a break. This initial 10-day course cured $49(49 \%)$ of these patients, and ultimately the ulcers of $94(93 \%)$ healed on this low-dose regimen. In seven of these patients treatment with sodium stibogluconate was stopped, and they were given alternative treatment because of dissatisfaction with their clinical progress. The ulcers of $90 \%$ of patients treated with this low-dose regimen had healed after a cumulative dose of $24 \mathrm{~g}$ (i.e. 40 days' treatment).

The higher dose sodium stibogluconate regimen $(600$ $\mathrm{mg}$ twice daily) was initiated in 72 soldiers. Treatment was given for 14 days in the first instance, after which it was either continued, or repeated after a break. The initial 14-day course cured $46(63.9 \%)$ of these patients, and ultimately the ulcers of all these patients healed on this regimen. The ulcers of $90 \%$ of patients treated with this regimen had clinically healed after a cumulative dose of $24 \mathrm{~g}$ sodium stibogluconate (i.e. 20 days' treatment).

Seven patients relapsed following healing of their lesions. In six cases this occurred after an interval of between 4 and 7 months, and in the seventh case after 19 months. Six were successfully re-treated with sodium stibogluconate, and in the remaining case the lesion was excised. Mucocutaneous leishmaniasis has not, as yet, developed in any British soldier following service in Belize.

\section{Discussion}

There are few reports which document the clinical features of American cutaneous leishmaniasis, ${ }^{1.4-6}$ but that of Llanos Cuentas et al. ${ }^{6}$ describes the lesions in 182 cases with active disease. One of the problems with interpretation of data from previous studies is that the diagnosis was not confirmed by demonstration of the parasite in all cases. In this study, although a clinical diagnosis of CL was made in 306 cases, analysis was restricted to the 187 cases in which the parasite was demonstrated. In each patient a single lesion was biopsied, and it was assumed that other, clinically similar lesions in the same person, were of the same aetiology. Our observation that over $70 \%$ of cases presented with a solitary lesion. normally on the exposed extremities, is similar to other reports. However, in this study a greater proportion of lesions occurred on the hands and wrists compared with the legs. This may 
attributable to natives working in jungle areas with bare legs, whereas British troops wore jungle boots and long trousers. Also, a higher proportion of lesions due to $\mathrm{Lmm}$ occurred on the head and neck - this has been attributed to the biting habits of Lutzomyia olmeca olmeca, the vector of Lmm which tends to bite at head level. ${ }^{1}$ The vector of $\mathrm{Lbb}$ in Belize has not yet been established. The average diameter of lesions at presentation was smaller than reported in other studies, which is probably the result of earlier presentation. Medical officers and troops receive instruction about CL prior to a Belize tour of duty, and are given a 'Leishmaniasis warning card', resembling a credit card, upon their return, to show to any medical attendant they consult during the following 2 years. Referral for assessment to the Military Hospital in Aldershot is recommended.

Diagnosis may be difficult. In the early stages the lesion resembles an insect bite, and later the presence of secondary infection may lead to further diagnostic difficulty. Demonstration of the parasite is crucial to confirm the clinical diagnosis. Histological identification of the parasite by Giemsa staining, and culture, followed by typing, were the main methods employed. However, parasitological confirmation of the diagnosis was obtained in only $61 \%$ of cases. There is a need for more sensitive diagnostic tests-at present most centres rely on a battery of different diagnostic methods consisting of histology, culture, touch smears ${ }^{7}$ and serology. ${ }^{8}$

Lbb was identified from 77 cases, Lmm from a further 29 , and in one additional case both were identified from a single lesion. As it is more difficult to culture Lbb than Lmm, which grows readily, ${ }^{8}$ it is likely that Lbb is the most prevalent type of $\mathrm{CL}$ in Belize. It is therefore imperative that all such cases should receive effective systemic therapy to prevent the subsequent development of metastatic mucocutaneous disease. ${ }^{9}$ The identification of Lmm from a single lesion does not remove this obligation. Lesions due to Lbb and Lmm cannot be differentiated by their clinical features, although there is an indication that those due to Lmm grow more slowly.

This study confirms the relative safety and efficacy of pentavalent antimony compounds, which have an unjustifiably poor reputation because of the toxicity of the earlier trivalent antimony compounds. ${ }^{9}$ The manufacturer of sodium stibogluconate recommends a 10-day course of $600 \mathrm{mg}$ i.v. daily, which may be repeated after a 10-day interval. ${ }^{3}$ A single course cured $48.5 \%$ of our cases, with minimal side-effects. A higher dose regimen was subsequently introduced because of dissatisfaction with the high proportion of patients requiring further ourses of treatment, coupled with increased confidence in the safety profile of sodium stibogluconate. This regimen consisted of an initial 14-day course of sodium stibogluconate $600 \mathrm{mg}$ i.v. b.d. which was either continued, or repeated after a break, if ulcer healing was not satisfactory. This initial course cured $63.9 \%$ of cases, and all those treated with this regimen ultimately healed with sodium stibogluconate alone. It was notable that in both the high-and low-dose regimens $90 \%$ of patients were cured when $24 \mathrm{~g}$ of sodium stibogluconate had been administered. It is not possible to determine whether the seven patients withdrawn from the lowdose regimen would have ultimately healed if sodium stibogluconate had been continued, or whether there was true resistance to antimony at that dose. It may be significant that six of these seven patients had Lmm infections. The development of antimony resistance is a cause for concern, and has been linked to inadequate initial daily doses and treatment durations. ${ }^{9}$

The higher dose regimen was associated with greater clinical morbidity. The fall in white cell count and rise in both transaminases and alkaline phosphatase levels which are objective evidence of toxicity, although a feature of both regimens, were of greater magnitude in those receiving the higher dose. Higher dose regimens have been developed by others ${ }^{5,10}$ but dose-dependent toxicity was not reported. However, there was a large individual variation apparent in these biochemical and haematological changes, which were reversible after cessation of treatment, and usually developed in the absence of clinical symptoms. It is unlikely, however, that factors other than the therapy caused these changes, as patients were confined to hospital during treatment. The only cardiac effect noted in a group of 22 of these patients, who had received the low-dose regimen, was a reversible flattening of T-wave amplitude. ${ }^{11}$ We did not study the cardiac effects of the higher dose regimen, but they have been reported to be dose-related by others studying stibogluconate therapy in visceral leishmaniasis. ${ }^{12}$

In our experience sodium stibogluconate $600 \mathrm{mg}$ i.v. twice daily for 14 days, with an option to extend or repeat the course if necessary, is an appropriate regimen for the treatment of American CL and can be expected to cure all cases. The initial 14-day course cured $63.9 \%$ of cases, and if extended to 20 days a cure rate of $90 \%$ would be anticipated. This higher dose regimen is associated with greater morbidity, but the development of drug resistance is less likely to become a clinical problem if higher dose regimens are given in the first instance for a reasonable period of time. Other types of treatment have been tried on an ad hoc basis, but none 
has been found as useful, and none formally evaluated.

All our cases were followed up for at least 3 months by Army dermatologists. All were servicemen who were then followed up by their regimental medical officer, and it is unlikely that any relapses would have escaped our notice. No cases of mucocutaneous leishmaniasis have occurred. In the light of the pattern of relapse we have observed, it would seem appropriate to keep patients with CL under review for at least 2 years.

\section{Acknowledgments}

We thank Col. D.Sim-Davis, Col. D.S.Jolliffe and Lt. Col. K.Freeman for encouragement to report their patients, Dr D.A.Evans for culture and isoenzyme analyses, Dr A.Bryceson for advice on patient management, and $\mathrm{Dr}$ R.A.Elton for statistical advice.

\section{References}

1 Walton BC. American cutaneous and mucocutaneous leishmaniasis. In: The Leishmaniases in Biology and Medicine (Peters W, KillickKendrick R., eds). London: Academic Press, 1987: 637-64.

2 Evans DA. Lanham SM. Baldwin CI, Peters W. The isolation and isoenzyme characterization of Leishmania braziliensis subsp from patients with cutaneous leishmaniasis acquired in Belize. Trans $R$ Soc Trop Med Hyg 1984; 78: 35-42.

3 Anonymous. Pentostam data sheet. In: ABPI Data Sheet Compendium 1989-1990. London: Datapharm Publishers.

4 Jones TC, Johnson WD Jr, Barretto AC et al. Epidemiology of American cutaneous leishmaniasis due to Leishmania braziliensis braziliensis. J Infect Dis 1987; 156: 78-83.

5 Ballou WR, McClain JB, Gordon DM et al. Safety and efficacy of high-dose sodium stibogluconate therapy of American cutaneous leishmaniasis. Lancet 1987: ii: 13-16.

6 Llanos Cuentas EA, Cuba CC. Barreto AC, Marsden PD. Clinical characteristics of human Leishmania braziliensis braziliensis infections. Trans R Soc Trop Med Hyg 1984: 78: 845-6.

7 Berger RS, Perez-Figaredo RA. Spielvogel RL. Leishmaniasis: the touch preparation as a rapid means of diagnosis. J Am Acad Derm 1987: 16: 1096-1105.

8 Manson-Bahr PEC. Diagnosis. In: The Leishmaniases in Biology and Medicine (Peters W. Killick-Kendrick R., eds). London: Academic Press, 1987: 703-72.

9 Bryceson ADM. Therapy in man. In: The Leishmaniases in Biology and Medicine (Peters W. Killick-Kendrick R., eds). London: Academic Press, 1987: 847-907.

10 Oster CN, Chulay JD, Hendricks LD et al. American cutaneous leishmaniasis: a comparison of three sodium stibogluconate treatment schedules. Am J Trop Med Hyg 1985: 34: 856-60.

11 Henderson A, Jolliffe D. Cardiac effects of sodium stibogluconate. Br J Clin Pharmacol 1985: 19: 73-7.

12 Chulay JD. Spencer HC, Mugambi M. Electrocardiographic changes during treatment of leishmaniasis with pentavalent antimony (sodium stibogluconate). Am J Trop Med Hyg 1985; 34: 702-9. 
We are grateful to Dr Tribble for reviewing the manuscript and making the necessary comments and suggestions. We also thank Ms Magda Erian for data collection and analysis, Ms Amal El-Dessouky for secretarial work, and Research Publication staff members for reviewing the manuscript.

\section{References}

Baker, L. H. \& Baker, A. B. (1981). Non viral forms of encephalitis. In: Clinical Neurology, vol. 2, Baker, A. B. \& Baker, L. H. (editors). New York: Harper \& Row, pp. 1213.

Fishman, R. A. (1982). Steroids in the treatment of brain oedema. New England fournal of Medicine, 306, 359-360.

Ghand, G. \& Singh, K. (1988). Acute cerebellar ataxia, a rare complication of enteric fever. Fournal of the Association of Physicians of India, 36, 741-742.

Hoffman, S. L., Punjabi, N. H., Kumala, S., Moechtar, A., Pulungsih, S. P., Rivai, A. R., Rochhell, R. C., Woodward, T. E. \& Loedin, A. A. (1984). Reduction of mortality in chloramphenicol-treated severe typhoid fever by high dose dexamethasone. New England fournal of Medicine, 310, 8288 .
Osuntokun, B. D., Bademosi, O., Ogunremi, K. \& Wright, S. G. (1972). Neuropsychiatric manifestations of typhoid fever in 959 patients. Archives of Neurology, 27, 7-13.

Sawhney, I. M., Prabhakar, S., Dhand, U. K. \& Chopra, J. S. (1986). Acute cerebellar ataxia in enteric fever. Transactions of the Royal Society of Tropical Medicine and Hygiene, 80, 8586.

Ukadgoankar, N. G., Talib, S. H., Kharkar, R. A. \& Ekbole, S. P. (1981). Acute reversible cerebellar syndrome in enteric fever. Foumal of the Association of Physicians of India, 29, $781-782$.

Verma, P. S., Mullich, P. \& Ghosh, S. (1972). Neurological manifestations of enteric fever-clinical profile and correlation with ultimate outcome. Indian Paediatrics, 9, 681-685.

Wadia, R. S., Ichaporia, N. R., Kuvalkar, R. S., Amin, R. B. \& Sardesai, H. V. (1985). Cerebellar ataxia in enteric fever. fournal of Neurology, Neurosurgery and Psychiatry, 48, 695697.

Received 1 April 1993; accepted for publication 29 April 1993

\section{Short Report \\ Thrombocytopenia complicating sodium stibogluconate therapy for cutaneous leishmaniasis}

\section{N. C. Hepburn University Department of Dermatology,} The Royal Infirmary, Edinburgh, EH3 9YW, UK

Sodium stibogluconate is the mainstay of therapy for American cutaneous leishmaniasis. Patients frequently report anorexia, malaise and myalgia during therapy. A transient leucopenia, a rise in serum transaminase levels (BALlou et al., 1987; HEPBURN et al., 1993) and electrocardiographic abnormalities (CHULAY et al., 1985) have also been reported and these appear to be dose related. Thrombocytopenia has not been reported.

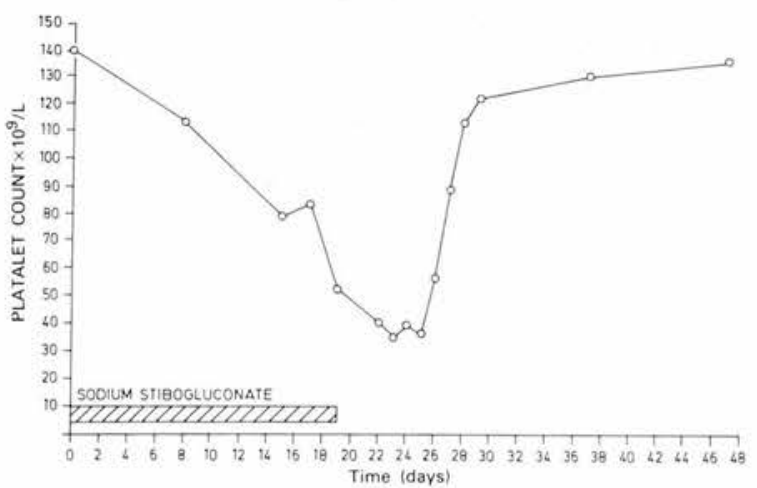

Figure. Platelet count changes with time during and after sodium stibo gluconate therapy for cutaneous leishmaniasis.

A 24 years old British soldier contracted cutaneous leishmaniasis during 6 months duty in Belize. $\mathrm{He}$ resented 4 months after his return to the UK with a palle surmounted by a crust measuring $1 \mathrm{~cm}$ in diameter on his left forearm. The crust had separated on several occasions but always reformed. The diagnosis was confirmed by finding amastigotes in a biopsy; culture was negative.

Once haematological and biochemical baseline values had been established, treatment was started with sodium stibogluconate (Pentostam ${ }^{\circledR}$, Wellcome, UK) $20 \mathrm{mg} / \mathrm{kg} / \mathrm{d}$ ( $1.7 \mathrm{~g}$ daily). His platelet count progressively fell during treatment, which was stopped after $19 \mathrm{~d}$. His platelet count reached a nadir of $36 \times 10^{9} / \mathrm{L} 4 \mathrm{~d}$ after his last dose and did not begin to recover until $8 \mathrm{~d}$ after his treatment ended (see Figure). His white blood cell count fell from $5.8 \times 10^{9}$ to $3.1 \times 10^{9} / \mathrm{L} 3 \mathrm{~d}$ after treatment had been stopped. There was no change in his haemoglobin concentration.

Sodium stibogluconate is now used at higher doses in courses of longer duration than were common a few years ago. This change has led to better cure rates at the cost of greater morbidity (HEPBURN et al., 1993). Transient leucopenia during the first week of treatment is well documented (BALLOU et al., 1987; HEPBURN et al., 1993) but, although I have noted a fall in the platelet count during therapy in other patients, the absolute count has remained within the normal range. This patient developed severe thrombocytopenia yet he was asymptomatic and haemostatic. The case illustrates the importance of weekly platelet counts so that sodium stibogluconate can be stopped before haemorrhagic complications ensue.

\section{References}

Ballou, W. R., McClain, J. B., Gordon, D. M., Shanks, G. D., Andujar, J., Berman, J. D. \& Chulay, J. D. (1987). Safety and efficacy of high dose sodium stibogluconate therapy of American cutaneous leishmaniasis. Lancet, ii, 13-16.

Chulay, J. D., Spencer, H. C. \& Mugambi, M. (1985). Electrocardiographic changes during treatment of leishmaniasis with pentavalent antimony (sodium stibogluconate). American fournal of Tropical Medicine and Hygiene, 34, 702-709.

Hepburn, N. C., Tidman, M. J. \& Hunter, J. A. A. (1993). Cutaneous leishmaniasis in British troops from Belize. British fournal of Dermatology, 128, 63-68.

Received 4 May 1993; accepted for publication 19 May 1993 


\title{
$\gamma \delta$ T cells in the peripheral blood of individuals from an area of holoendemic Plasmodium falciparum transmission
}

\author{
Martin Goodier ${ }^{1}$, Maria Krause-Jauer ${ }^{1}$, Ambaliou Sanni ${ }^{2}$, Achille Massougbodji ${ }^{3}$, Benoît-Christophe Sadeler ${ }^{3}$, \\ Graham H. Mitchell ${ }^{4}$, Manuel Modolell ${ }^{1}$, Klaus Eichmann ${ }^{1}$ and Jean Langhorne ${ }^{1^{\star}}{ }^{1}$ Max-Planck-Institut für \\ Immunbiologie, Stübeweg 51, D-7800 Freiburg, Germany; ${ }^{2}$ Départment de Biochimie et Biologie Cellulaire, Faculté des \\ Sciences et Techniques, Université Nationale du Bénin, Cotonou, Bénin; ${ }^{3}$ Départment de Parasitologie, Faculté des Sciences de \\ la Santé, Université Nationale du Bénin, Cotonou, Bénin; ${ }^{4}$ Department of Immunology, United Medical and Dental Schools, \\ Guy's Hospital Medical School, London, SE1 9RT, UK
}

\begin{abstract}
$\gamma \delta \mathrm{T}$ cells bearing $\mathrm{V} \gamma 9 \mathrm{~T}$ cell receptors from unexposed Caucasian donors make large responses to Plasmodium falciparum in vitro. This finding, together with observations of others showing high levels of $\mathrm{V} \gamma 9^{+} \mathrm{T}$ cells in the blood of infected non-immune individuals, led us to hypothesize that the response of these cells might contribute to the pathology of $P$. falciparum malaria. Acquisition of immunity to disease in people naturally exposed to infection may therefore be due in part to down-regulation or alteration of the function of $\gamma \delta \mathrm{T}$ cells. Supporting this view, and in contrast to infection in non-immune individuals, $\mathrm{V} \gamma 9^{+} \mathrm{T}$ cells are not elevated in peripheral blood of children or adults living in an endemic area despite constant exposure to $P$. falciparum. After in vitro stimulation with $P$. falciparum, however, the expansion of $\mathrm{V} \gamma 9^{+}$cells from the African donors is of similar magnitude to that observed for non-exposed Europeans. Thus, although these cells are not elevated in peripheral blood, they are still able to respond to $P$. falciparum antigens. In adult European donors the major $\gamma \delta \mathrm{T}$ cell population in peripheral blood is $\mathrm{V} \gamma 9^{+}$(approximately $70 \%$ of all $\gamma \delta$ cells), whereas in the majority of adult Africans ${\mathrm{V} \delta 1^{+} \mathrm{V} \gamma 9^{-}}^{-} \mathrm{T}$ cells predominated (approximately $70 \%$ of total $\gamma \delta$ cells).
\end{abstract}

\section{Introduction}

$\gamma \delta \mathrm{T}$ cells bearing V $\gamma 9 \mathrm{~T}$ cell receptors collected from donors not previously exposed to malaria, mount rapid responses to antigens of Plasmodium falciparum in vitro (GOERLICH et al., 1991; BEHR \& DUBOIS, 1992; GOODIER et al., 1992). In addition, in individuals acutely infected with $P$. falciparum, the proportion of these cells is increased in the peripheral blood (Ho et al., 1990; ROUSSILHON et al., 1990; CHANG et al., 1992). Upon activation, cells of the $\gamma \delta$ T cell lineage can produce a variety of lymphokines such as interleukin 2, interferon $\gamma$ (IFN- $\gamma$ ), and tumour necrosis factors $\alpha$ and $\beta$ (TNF- $\alpha$ and TNF- $\beta$ ) (CRON et al., 1989; CHRISTMAS \& MEAGER, 1990; BIASSONI et al., 1991; MORITA et al., 1991; FOL. LOWS et al., 1992), some of which are responsible for the activation of macrophages with subsequent induction of inflammatory processes. Since these cells from non-exposed individuals respond at a high frequency and have been found in increased numbers of the peripheral blood of infected non-immune individuals, we have hypothesized that the activation of these cells may contribute to the fever and pathology associated with $P$. falciparum malaria (LANGHORNE et al., 1992). In endemic areas of $P$. falciparum, individuals are exposed constantly to the parasite and therefore $\gamma \delta \mathrm{T}$ cells could be continuously stimulated. However, it is well established that in these areas severe complications of malaria decrease with age, and older individuals with parasitaemia show little evidence of pathological complications. This generally occurs before acquisition of immunity to infection (MCGREGOR \& Wilson, 1988; Playfair et al., 1990). Therefore, if $\gamma \delta \mathrm{T}$ cells play a role in pathology, they must either no longer respond to $P$. falciparum or have an altered response pattern in older immune individuals. This may then be reflected in a lack of elevation of circulating $\gamma \delta \mathrm{T}$ cells and/or an inability of $\mathrm{V} \gamma 9^{+}$cells to respond to $P$. falciparum in vitro. In order to test this hypothesis, we determined whether $\gamma \delta \mathrm{T}$ cells were elevated in the peripheral blood of children and adults living in an area of holoendemic $P$. falciparum. The proportions of the subsets of peripheral blood $\gamma \delta \mathrm{T}$ cells were monitored before and after stimulation in vitro with erythrocytes infected with $P$. falciparum.

\section{Materials and Methods}

Subjects

Blood was taken from 18 adults ( $>20$ years old), and from 17 children ( $<10$ years old) living in and around Cotonou, Benin, West Africa, during 1990. In this region malaria is holoendemic with maximal transmission during the 2 rainy seasons (HOUNTONDJI et al., 1989). Thin and thick Giemsa-stained blood films were examined for parasites at the time of sampling.

\section{Preparation, freezing and recovery of peripheral blood mono-} nuclear cells

Peripheral blood mononuclear cells (PBMC) were prepared by centrifugation over Ficoll-Hypaque ${ }^{\circledR}$ (Pharmacia, Freiburg, Germany), according to standard procedures. Supernatants were then taken from the gradients and reserved frozen as 'plasma' for subsequent antibody determinations. Mononuclear cells were removed and washed twice in phosphate-buffered saline (PBS) before freezing at $5 \times 10^{6} / \mathrm{mL}$ in RPMI 1640 medium, $10 \%$ dimethyl sulphoxide and $20 \%$ foetal calf serum at $-80^{\circ} \mathrm{C}$ overnight and thereafter in liquid nitrogen. For recovery, cells were transferred to a large volume of warm medium, centrifuged, and washed twice in fresh medium before counting. More than $50 \%$ of the frozen cells were normally recovered. Freezing and thawing of PBMC did not alter the distribution of the different subsets of cells. The expression of the surface molecules $\alpha \beta, V \gamma 9^{+}$and V81-TCR, CD4, CD8, CD45RO, CD45RA, CD14 and CD20 (see section on flow cytometric analysis) on frozen and thawed PBMC measured before or after in vitro stimulation with $P$. falciparum was not significantly different from that on freshly isolated PBMC from the same donors.

\section{Cell culture and proliferation assays}

Cells were cultured at $5 \times 10^{5} / \mathrm{mL}$ in 96-well plates in RPMI 1640 medium with $15 \%$ pooled human group A serum for $6 \mathrm{~d}$ in the presence of $1 \times 10^{4}$ parasites/well of cultured $P$. falciparum, strain $\mathrm{Kl}$ (origin in Thailand, kindly provided by $\mathrm{H}$. Matille, Hoffman-La Roche, Basel, Switzerland) or an equivalent number of control uninfected erythrocytes (GOODIER et al., 1992). For flow cytometric analysis, viable PBMC were isolated after $6 \mathrm{~d}$ of culture by Ficoll-Hypaque ${ }^{\circledR}$ centrifugation.

\section{Flow cytometric analysis}

The following monoclonal and polyclonal antibodie were used. Unlabelled antibodies: BMA031, anti- $\alpha$ TCR (mouse immunoglobulin (Ig) $\mathrm{G}_{2 \mathrm{~b}}$, kindly provid? by R. Kurrle, Behring, Marburg, Germany); OKN anti-CD14 (mouse IgG 2 b, American type tissue culy 


\title{
The management of American cutaneous leishmaniasis
}

\author{
NC Hepburn \\ University Department of Dermatology, The Royal Infirmary, Edinburgh, UK
}

\begin{abstract}
American cutaneous leishmaniasis is a major world health problem which is encountered more frequently in the Western world. The clinical features, diagnosis and treatment are described. Sodium stibogluconate is the mainstay of therapy, and should be administered in a dose of $20 \mathrm{mg} / \mathrm{kg}$ per day for 28 days. It is associated with leucopenia, elevation in the serum transaminases and ECG $\mathrm{T}$-wave changes. However, the drug appears safe in clinical practice. (J Dermatol Treat (1992) 3: 95-98)
\end{abstract}

\section{Introduction}

The leishmaniases are major protozoal diseases affecting approximately 12 million people worldwide. ${ }^{1}$ They are transmitted by the bite of an infected sandfly, and produce three principle clinical syndromes: visceral leishmaniasis (VL), cutaneous leishmaniasis (CL) and mucocutaneous leishmaniasis (MCL). The form of leishmaniasis which develops is determined principally by the species of the infecting parasite (Figure 1). This review is limited to American ('New World') CL, a disease which is rapidly increasing in incidence. ${ }^{2}$ It is caused by either the Leishmania mexicana complex, which causes a simple cutaneous ulcer, or the L. braziliensis complex which also causes a cutaneous ulcer initially but may progress to the destructive mucocutaneous form ('Espundia') by metastatic spread. MCL may develop shortly after the ulcer, but its appearance may be delayed for over 20 years. ${ }^{2}$

In 1978 the British army established a permanent garrison of approximately 1500 troops in Belize. Most soldiers are on 6-month tours of duty and undertake jungle training during which they are exposed to the bites of Lutzomyia sandflies. Between 1978 and mid 1990, 306 soldiers were treated for $\mathrm{CL}$ following a tour of duty in Belize. Prior to 1982 all reported cases of Belizian CL were due to the L. mexicana complex. However, the L. braziliensis complex, with its associated risk of late $\mathrm{MCL}$, has subsequently been identified in many of the returning soldiers. ${ }^{3}$

\section{Clinical features}

The lesion starts as a small inflammatory papule which gradually enlarges and ulcerates. An adherent crust initially covers the ulcer but this separates to expose a necrotic base which is frequently secondarily infected vith either Staphylococcus aureus or B-haemolytic strep-

spondence: Dr NC Hepburn, University Department of tology, The Royal Infirmary, Edinburgh EH3 9YW, UK tococci. The fully developed lesion consists of a painless ulcer with a necrotic base and an indurated violaceous margin (Figure 2). Most lesions occur on exposed body sites (Figure 3). In an analysis of 187 parasitologicallyproven cases from Belize the mean diameter of the ulcers was $14.4 \mathrm{~mm}$ (SD 9.3) after an average duration of 9.9 weeks (SD 6.7), and a single lesion was present in $71 \%$ of cases with only $1 \%$ having more than four lesions. Infected insect bites are the most common problem in differential diagnosis but these are usually more numerous. Other differential diagnoses include infected traumatic ulcers, mycobacterial or fungal infections, tropical ulcers and neoplastic ulcers. It is not possible to distinguish between lesions caused by the $L$. braziliensis complex and the L. mexicana complex on the basis of the clinical picture.

\section{Diagnosis}

The diagnosis of CL requires a high index of suspicion in patients presenting with an ulcer and an appropriate travel history. Demonstration of the parasite represents the diagnostic gold standard and this must always be attempted. A biopsy from the active edge of the ulcer encompassing the full thickness of the dermis should be divided into three parts. First, an impression smear should be prepared in which the fresh biopsy is pressed against the surface of a microscope slide two or three times. This is fixed with methyl alcohol for $3 \mathrm{~min}$ and then stained using the Giemsa technique. ${ }^{4}$ Leishman-Donovan (LD) bodies, which represent the leishmania amastigotes, appear as round to oval bodies of $2-6 \mu \mathrm{m}$ in length, and are most easily identified in the intracellular space. ${ }^{5}$

The second part of the biopsy should be processed in paraffin and examined after staining with Giemsa and haematoxylin and eosin. In early lesions it is usually easy to identify the characteristic LD bodies. However, after a few months their numbers decrease and a granulomatous infiltrate develops. ${ }^{6}$ The final part of the biopsy should be used to culture the organism. This requires complex media and some expertise involving the use of a modified NNN medium with antibiotics to prevent bacterial overgrowth. Once the organism has been cultured the infecting species can be identified by isoenzyme electrophoresis. All these techniques are clearly described in a handbook by Evans. ${ }^{4}$ In practice the Department of Medical Parasitology at the London School of Hygiene and Tropical Medicine operates an excellent leishmania identification service.

The Montenegro leishmanin skin test, which resembles the tuberculin test, is a useful supplementary diagnostic procedure in which $0.1 \mathrm{ml}$ of a suspension containing $10^{5}$ promastigotes in $0.5 \%$ phenol saline is injected intradermally into the forearm. An equal amount of $0.5 \%$ phenol 


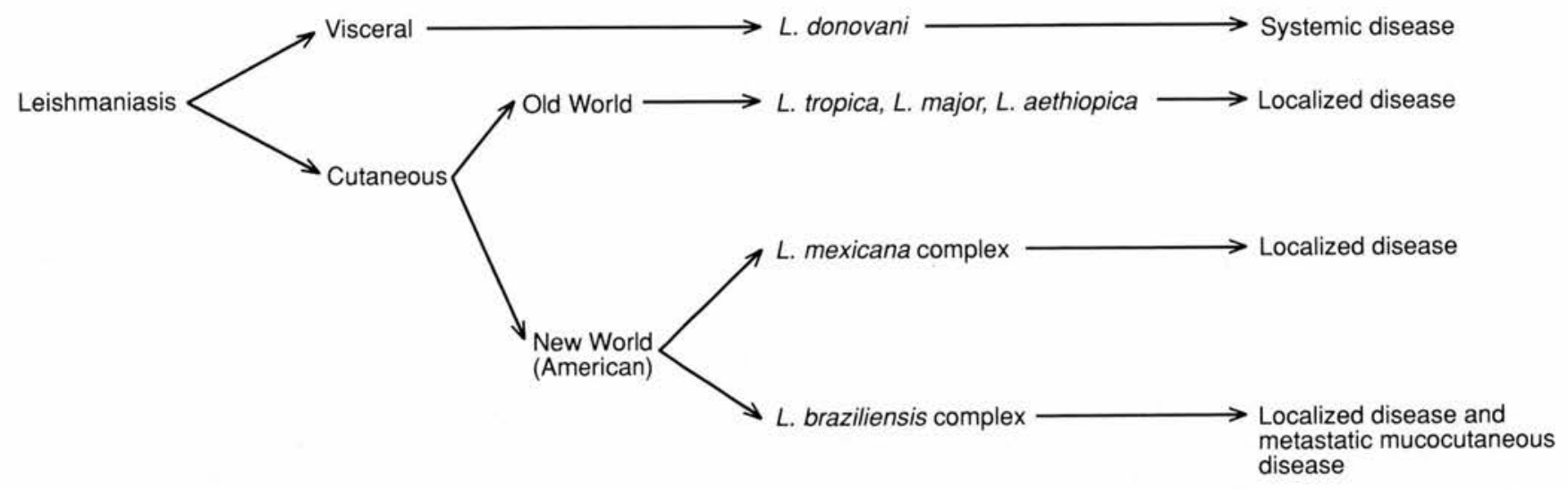

Figure 1 Principle types of leishmaniasis.

saline alone is injected into a second, adjacent, site as a control to determine sensitivity to the diluent. ${ }^{7}$ A delayed hypersensitivity reaction develops, consisting of an indurated nodule with surrounding erythema. This reaction is read at $48-72 \mathrm{~h}$. A positive result is represented by an area of induration measuring more than $5 \mathrm{~mm}$ in diameter which can be graded: grade 1,5-6 mm; grade 2, 6-8 $\mathrm{mm}$; grade $3,>8 \mathrm{~mm}$; and grade 4 , with blistering. ${ }^{8}$

Unfortunately, standardized test antigens are not available. The reagents are produced in the laboratories of tropical disease centres and vary in their sensitivity and specificity. ${ }^{9}$ More than $90 \%$ of patients with CL give a positive reaction, ${ }^{5}$ but so do up to $9 \%$ of healthy controls in endemic areas. ${ }^{9}$ We have not found serological diagnostic procedures sufficiently specific to help in diagnosis.

\section{Treatment}

The literature on the treatment of American CL is fraught with contradiction, misconceptions and anecdotal reports of alternative therapies. The first crucial step in management is to decide whether or not one of the $L$. braziliensis complex may be the infecting organism because, until further information is available, all such cases must be considered capable of causing MCL, and therefore adequate systemic treatment is mandatory. If the patient is thought to have contracted the disease in any of the areas in which the L. braziliensis complex occurs (i.e. Belize, Honduras, Costa Rica, Panama, Venezuela, Colombia, Suriname, French Guiana, Brazil, the Western slopes of the Peruvian Andes and the Argentinian Highlands),${ }^{10}$ the parasite must be isolated and identified before any alternative to optimal systemic treatment can be considered. If the disease was contracted in other areas, or if the disease is shown to be due to the $L$. mexicana complex, alternatives, including topical treatment or no treatment at all, may be considered.

Pentavalent antimony remains the mainstay of effective systemic treatment for CL. These compounds have been widely used since the 1940s, yet have an unjustifiably poor reputation which appears to stem from their toxic predecessors, the trivalent antimony compounds. In our hands, and those of others, these compounds have been remarkably safe. ${ }^{11,12}$ In the UK, and now in the US, ${ }^{13}$ the treatment of choice is sodium stibogluconate (Pentostam) given by slow intravenous injection. The optimal treat- ment regimen has not yet been determined, but the manufacturer's recommendation of $600 \mathrm{mg}$ daily for 10 days (total dose $6 \mathrm{~g}$ ), followed by a 10-day rest period after which further courses may be administered ${ }^{14}$ is suboptimal. We have found this regimen cures only $48.5 \%$ of cases after the first course, although it is associated with little morbidity.

There has therefore been a move to higher dose regimens. In the British army we changed to $600 \mathrm{mg}$ twice daily for 14 days with an option to continue, or repeat the course after a break, if necessary. The initial 14-day course cured $63.9 \%$ of cases, and all 72 patients started on this regimen eventually healed without any other antileishmanial therapy, although one case required a total of $51 \mathrm{~g}$. We have recently extended our initial course to 20 days which we expect will cure at least $90 \%$ of patients first time. The British army is unusual in using a fixed-daily dose regimen. This is acceptable because all of our patients have been well nourished adult males. However, because the majority of cases occur in the developing world, most authorities recommend a dose adjusted for body weight, and the current World Health Organization recommendation is to give $20 \mathrm{mg} / \mathrm{kg}$ per day for 28 days. ${ }^{\prime}$

These higher dose regimens are associated with more morbidity including anorexia, myalgia, arthralgia, chest wall pain and general malaise. However, these symptoms resolve spontaneously when treatment is stopped. There is usually a marked rise in the serum transaminases and a mild leucopenia during treatment, but these laboratory parameters do not correlate with subjective complaints of malaise and revert to normal when treatment stops. Non-specific T-wave changes have been documented during treatment, but no objective evidence of cardiotoxicity has been found by us, and in general this drug appears to have little cardiotoxicity. ${ }^{1,15}$ In a retrospective survey of 289 British soldiers treated with sodium stibogluconate, in only one case was it necessary to stop treatment because of toxicity - this patient developed an erythrodermic reaction. It is our practice to monitor the white blood cell count, urea, creatinine, liver function tests and to perform an ECG prior to treatment and then at weekly intervals during treatment. Tests with abnormal resul are then repeated at appropriate intervals until return to normal.

An alternative pentavalent antimony, commonly in Latin America, is meglumine antimoniate (Gl) 


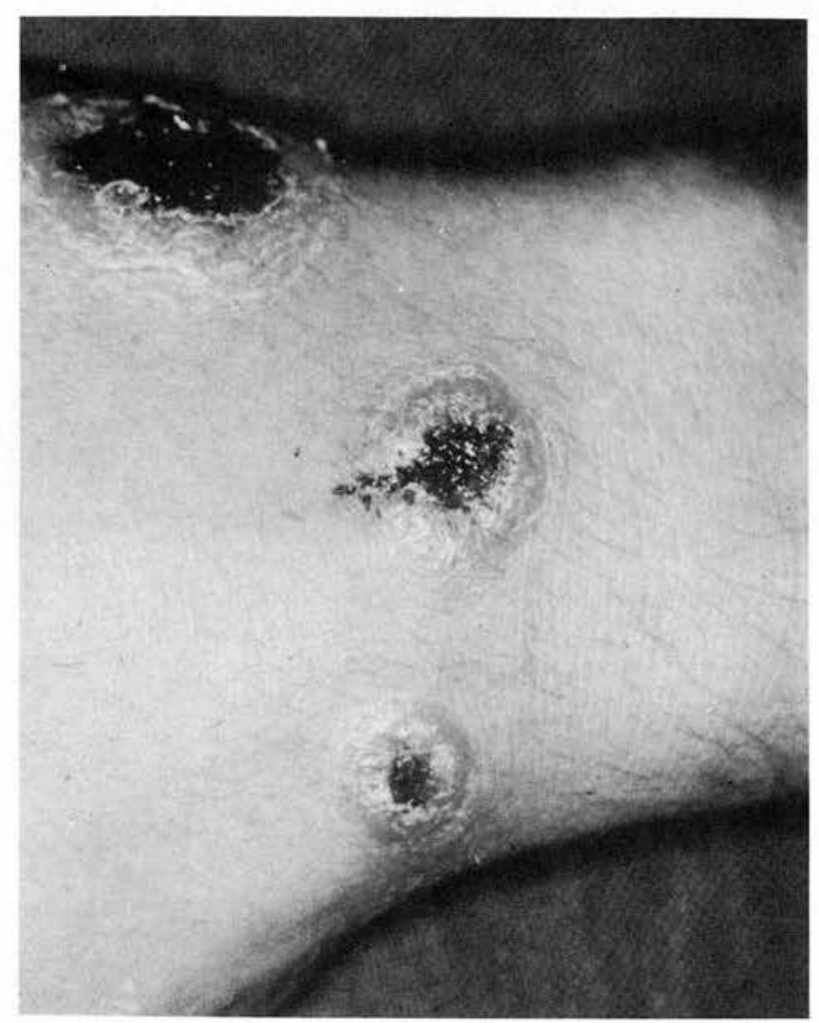

b

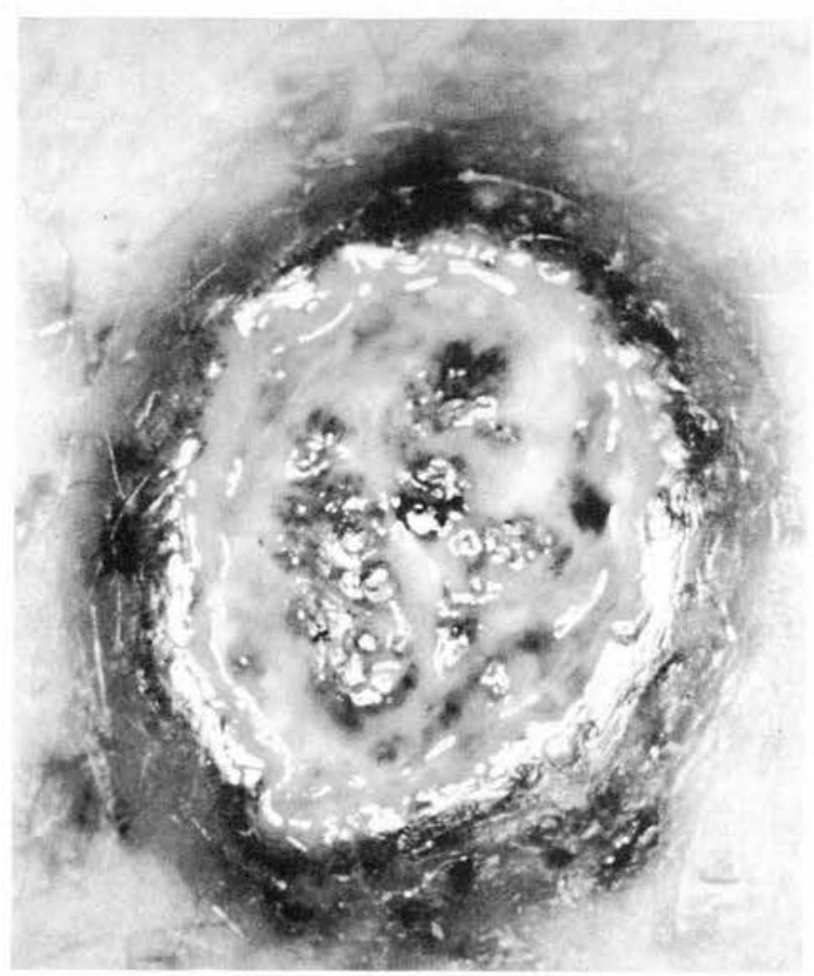

Figure 2a,b Cutaneous leishmaniasis of the hand.

fime). It is similar in efficacy and toxicity to sodium ibogluconate, but contains a lower concentration of timony $(85 \mathrm{mg} / \mathrm{ml}$ compared to $100 \mathrm{mg} / \mathrm{ml})$ so larger imes are required. Both preparations may be admini$\mathrm{d}$ as slow intravenous infusions or intramuscularly agh the volumes involved $(600 \mathrm{mg}=6 \mathrm{ml})$ make the ute uncomfortable.

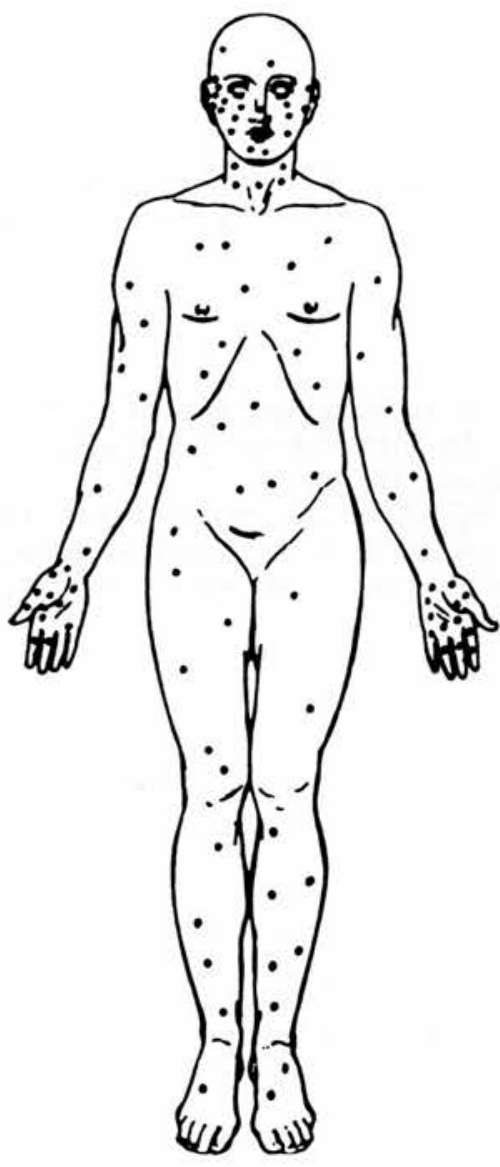

Figure 3 Site of 283 lesions in 187 British soldiers with parasitologically confirmed CL $(\mathbf{O}=1 \%$ lesions $)$.

Since adopting the higher dose regimen of sodium stibogluconate, we have found second-line treatments unnecessary. A preliminary trial of ketoconazole $400 \mathrm{mg}$ twice daily for 28 days cured all eight patients, ${ }^{16}$ but, subsequent experience was less encouraging. In patients sensitive or resistant to sodium stibogluconate, amphotericin B or pentamidine are suitable alternatives. ${ }^{17}$ Amphotericin B in a dose of $1 \mathrm{mg} / \mathrm{kg}$ body weight is given as an intravenous infusion in $5 \%$ dextrose over $4 \mathrm{~h}$ on alternate days for 2 months. Pentamidine, in a dose of $4 \mathrm{mg} / \mathrm{kg}$ body weight, is given by intramuscular injection on alternate days for 5 weeks in the first instance. 'The persistent forms of $\mathrm{CL}$, such as diffuse $\mathrm{CL}$, are not well understood and these patients should be referred to specialist centres for full assessment.

$\mathrm{CL}$ due to the L. mexicana complex is not associated with MCL and therefore may be managed in a similar manner to Old World CL. Active treatment is not absolutely necessary as most lesions will heal spontaneously, albeit with scarring, within 12 to 18 months. ${ }^{2}$ However, because of functional and cosmetic considerations, we treat all our cases. If the lesion is small it may be removed by local excision or currettage. Alternatively, early non-inflamed lesions can be infiltrated with $1-3 \mathrm{ml}$ sodium stibogluconate. Infiltration must be thorough and produce complete blanching of the lesion base, and may be repeated once or twice if necessary at intervals of $1-2$ days. ${ }^{17}$ Paromomycin ointment is an exciting recent development as it can be used on an out-patient basis. Unfortunately, many patients find the present formula- 
tion too irritant. ${ }^{18}$ Cryotherapy, Grenz rays and local heat are also used in some centres. ${ }^{1}$ It is our policy to treat all cases of CL from Belize with systemic sodium stibogluconate in the first instance as the delay in waiting for culture and typing results would cause more morbidity than a timely course of systemic sodium stibogluconate.

In a retrospective study of 187 parasitologically proven

\section{References}

1. WHO Expert Committee. Control of the leishmaniases, Technical Report Series No. 793. World Health Organisation: Geneva, 1990.

2. Walton BC, American cutaneous and mucocutaneous leishmaniasis. In: Peters W, Killick-Kendrick R, (eds). The leishmaniases in biology and medicine, vol 2. Clinical aspects and control. Academic Press: London, 1987, p. 637.

3. Evans DA, Lanham SM, Baldwin CI et al, The isolation and characterization of Leishmania braziliensis subsp from patients with cutaneous leishmaniasis acquired in Belize. Trans R Soc Trop Med Hyg (1982) 78: 35-42.

4. Evans DA (ed.), Handbook on isolation, characterization and cryopreservation of Leishmania. UNDP/Worldbank/ WHO Special programme for TDR. World Health Organisation: Geneva, 1989.

5. Berger RS, Perez-Figaredo RA, Spielvogel RL, Leishmaniasis: the touch preparation as a rapid means of diagnosis. J Am Acad Dermatol (1987) 16: 1096-105.

6. Lever WF, Schaumburg-Lever G, Histopathology of the skin (Seventh edn). Lippincott: New York and London, 1990, p. 394.

7. Schnur LF, Jacobson RL, Parasitological techniques. In: Peters W, Killick-Kendrick R, (eds). The leishmaniases in biology and medicine, vol 1. Biology and epidemiology. Academic Press: London, 1987, p. 500.

8. Manson-Bahr PEC. Diagnosis. In: Peters W, KillickKendrick R, (eds). The leishmaniases in biology and medicine, vol 2. Clinical aspects and control. Academic Press: London, 1987, p. 703.

9. Weigle KA, Valderrama L, Arias Al et al, Leishmanin skin test standardisation and evaluation of safety, dose, storage, longevity of reaction and sensitisation. Am J Trop Med Hyg (1991) 44: 260-71. cases of CL in British soldiers, seven relapsed after a clinical cure. In six cases this occurred after an interval of 4-7 months and in the seventh case after 19 months. Patients should be followed up for 2 years following clinical cure, and relapses should be investigated and treated in the same way as primary lesions in the first instance.

10. Lainson R, Shaw JJ, Evolution, classification and geographical distribution. In: Peters W, Killick-Kendrick E, (eds). The leishmaniases in biology and medicine, vol 1. Biology and epidemiology. Academic Press: London, 1987, p. 1.

11. Bryceson A, Therapy in man. In: Peters W, KillickKendrick R, (eds). The leishmaniases in biology and medicine, vol 2. Clinical aspects and control. Academic Press: London, 1987, p. 847.

12. Ballou WR, McClain JB, Gordon DM et al, Safety and efficacy of high dose sodium stibogluconate therapy in American cutaneous Leishmaniasis. Lancet (1987) ii: 13-16.

13. Kalter DC, Cutaneous and mucocutaneous Leishmaniasis. Prog Dermatol (1989) 23(4): 1-11.

14. Anonymous, Pentostam data sheet. In: ABPI data sheet compendium 1989-1990. Datapharm Publishers: London, 1989.

15. Henderson A, Jolliffe DS, Cardiac effects of sodium stibogluconate. Br J Clin Pharmacol (1985) 19: 73-7.

16. Jolliffe DS, Cutaneous leishmaniasis from Belize - treatment with ketoconazole. Clin Exp Dermatol (1986) 11: $62-8$.

17. WHO Expert Committee. The leishmaniasis. Technical Report Series No.701. World Health Organisation: Geneva, 1984.

18. El-On J, Livishin R, Even-Paz et al, Topical treatment of cutaneous Leishmaniasis. J Invest Dermatol (1986) 87: $284-8$. 
Erratum: Replacement of misprinted article in previous issue

\section{CUTANEOUS LEISHMANIASIS}

\section{Part I: Clinical Features}

\section{N. C. Hepburn, Department of Dermatology, Royal Infirmary of Edinburgh}

The leishmaniases are ancient afflictions of man which are now recognised to be much more prevalent than had been considered likely 10 years ago. The World Health Organisation estimates that 12 million people worldwide are infected and that 350 million are exposed to infection. ${ }^{1}$ In the Old World leishmaniasis extends from the Mediterranean, through the Middle East to India, the southern countries of the former Soviet Union, China, and down into Africa. In the New World it is to be found from South America to southern Texas.

The British army has a long association with this disease. In 1885 Cunningham (1843-1914), a doctor in the Indian Medical Service first described the leishmanial amastigote from a case of Delhi boil and the disease is named after Lieutenant-General Sir William Boog Leishman (1865-1926) who described the same parasites from the viscera of a fatal case of kala-azar. More recently, army dermatologists have been treating a steady stream of soldiers who have developed the cutaneous form of the disease during jungle training in Belize. The principle types of leishmaniasis are detailed in Table 1, but this paper concentrates on the actue cutaneous forms of the disease. Aspects of the epidemiology and prevention will be presented in Part II.

\section{PATHOGENESIS AND PATHOLOGY}

The leishmaniases are due to a protozoa which is transmitted by the bite of an infected female sandlfy, generally Phlebotomus in the Old World and Lutzomyia or Psychodopygus in the New World (Fig 1). The inoculated promastigotes activate complement and thereby attract monocytes/macrophages to the area. Mature promastigotes (bearing surface antigens of $116 \mathrm{kdaltons}$ ) bind $\mathrm{C}_{3}$ and then attach to $\mathrm{C}_{3} \mathrm{~b}$ receptors on the macrophage and are phagocytosed whilst immature promastigotes are destroyed by complement mediated cytotoxicity. Once inside the macrophage, the promastigotes lose their flagellae and become amastigotes which multiply repeatedly by binary fission. Infected macrophages then burst, releasing their amastigotes which in turn infect other macrophages. The subsequent fate of the amastigotes depends upon parasite and host factors which are poorly understood. Viscerotrophic species, such as $L$. donovani, tend to migrate throughout the reticulo-endothelial system, whereas dermotrophic species, such as $L$. major, usually remain close to the inoculation site. Any spread of dermotrophic species tends to be late and only to adjacent skin (producing satellite lesions) or to lymphatics and regional lymph nodes. It is possible that dermotrophic species migrate as far as viscerotrophic species but are unable to survive in the internal milieu-for example $L$. donovani amastigotes are able to tolerate higher temperatures and resist complement mediated cytotoxicity. These phenotypic distinctions are not absolute. L. braziliensis is able to migrate to the oropharyngeal mucosa where it may remain dormant for many years before 
TABLE 1

Principal types of leishmaniasis

\begin{tabular}{lll}
\hline Type & Cause & Effect \\
Leishmaniasis & $\begin{array}{l}\text { L. donovani } \\
\text { L. tropica } \\
\text { L. major } \\
\text { L. aethiopica }\end{array}$ & Localised cutaneous disease \\
\hline Lew World & L. mexicana complex & Localised cutaneous disease \\
& L. braziliensis complex & $\begin{array}{l}\text { Localised cutaneous disease } \\
\text { but late metastatic mucocutaneous } \\
\text { disease 'Espundia' }\end{array}$ \\
\hline
\end{tabular}

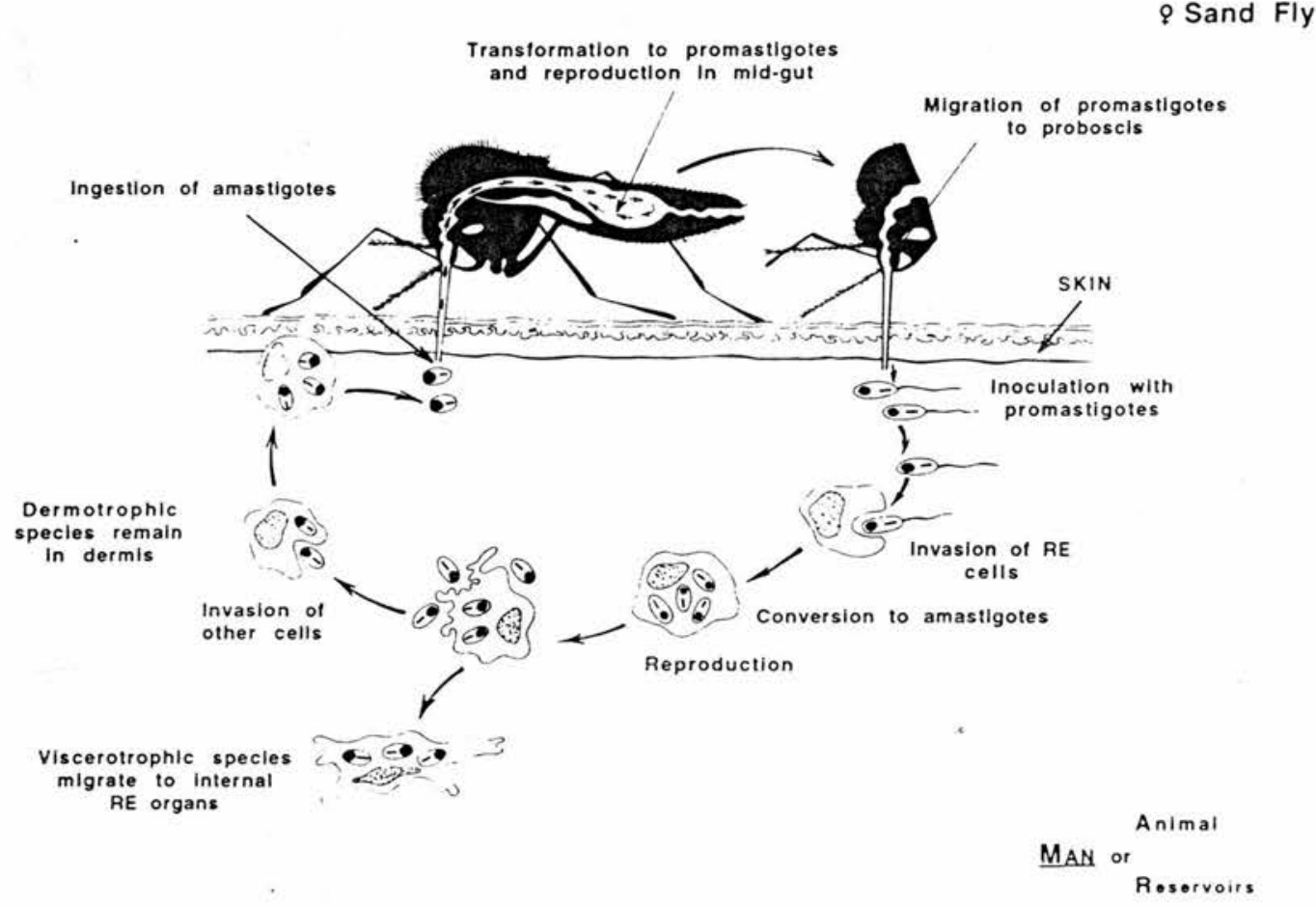

FIGURE 1

The life cycle of leishmania.

reactivating to cause the destructive mucocutaneous form of the disease 'Espundia'; there is at least one report of L. tropica causing visceral leishmaniasis ${ }^{2}$ and in post-kala-azar dermal leishmaniasis (PKDL) the viscerotrophic parasite becomes dermotrophic as a consequence of treatment.

The interaction between the patient's cell mediated immune response and the amastigotes is complex and results in a range of clinical and pathological changes. In all forms of leishmaniasis, however, the presence of amastigotes within the cells of the mononuclear phagocytic system remains the hallmark of the disease, 
although at times they may be difficult to detect. Infected macrophages measure $20-30 \mu \mathrm{m}$ in diameter whereas amastigotes, known as Leishman-Donovan (LD bodies), are round to oval structures measuring only $2-5 \mu \mathrm{m}$. They are surrounded by a plasma membrane and contain both a relatively large, deeply basophilic, nucleus and a smaller, densely staining, rod-shaped kinetoplast of extranuclear DNA (from the base of the flagellum which is lost in the conversion from promatigote to amastigote). Although haematoxylin and eosin can demonstrate amastigotes, a Giemsa stain that stains the kinetoplast bright red is preferred. ${ }^{3}$

In early cutaneous lesions, the dermal infiltrate mainly consists of macrophages filled with amastigotes. There are relatively few lymphocytes and plasma cells. As the lesions develop more lymphocytes and plasma cells appear and the superficial dermis becomes oedematous. The overlying epidermis becomes hyperkeratotic and subsequently breaks down to form an ulcer covered with a coagulum of hyperkeratotic debris, dried exudate, dead cells and a mixture of live and dead organisms. Over the following months there is a gradual decrease in the number of amastigotes and macrophages, leaving a granulomatous infiltrate consisting of lymphocytes, epitheloid cells and multinucleate-giant cells. At this stage it may be difficult or impossible to detect organisms in Giemsa sections. Parasite elimination usually follows destruction of the host macrophages, either at the centre of the circumscribed clusters in the dermis with the release of amastigotes, or in the subepidermal zone causing liquefaction of the basal layer and ulceration. Polymorphs are present in the necrotic zone and lymphocytes are numerous at the periphery. Ultimately, either the patient's immune response is able to eliminate the infection and effect a spontaneous cure or it fails and a chronic form of cutaneous leishmaniasis develops. Resolution occurs by fibrosis. ${ }^{1 \cdot 2.3}$

There are two special non-healing forms of cutaneous leishmaniasis (CL) which, although rare, are important because they represent two extremes of host cell-mediated immune (CMI) response to infection with leishmania and have given insights into the pathogenesis of this disease. Leishmaniasis recidivans (=lupoid) is characterised by healing with fibrosis in the centre of the lesion but failure to heal peripherally where there is a granulatomous infiltrate composed of epitheloid cells, multinucleate giant cells, a variable number of plasma cells but relatively few lymphocytes. Amastigotes are very scanty. The appearances are reminiscent of lupus vulgaris both histologically (although there is no caseation) and clinically (even apple jelly nodules are seen). The lesions usually occur on the face and develop from an acute lesion after an interval of 12-18 months. There is a central fibrotic scar surrounded by an active edge containing papules and nodules. In this type the parasite persists indefinitely despite an exaggerated host CMI response. It is found mainly in Iran and Iraq. The other extreme is represented by diffuse cutaneous leishmaniasis (DCL) in which there is a specific defect in the host CMI reponse to leishmania. Lesions contain a large number of macrophages full of amastigotes but lymphocytes are scarce. Clinically, the initial lesion spreads locally and then the disease disseminates to other areas of the body. There are numerous discrete papules and nodules which coalesce to form plaques scattered over the whole skin surface but particularly numerous on the face and the outer aspects of the limbs. DCL is associated with L. aethiopica in the Old World and L. mexicana complex in the New World; it is very resistant to treatment. 
TABLE 2

Ridley's classification of the histopathological response in cutaneous leishmaniasis.

\begin{abstract}
GROUP I Diffuse macrophage granuloma formation. Macrophages are full of amastigotes but do not show signs of activation. Occasional lymphocytes and plasma cells, but no giant cells. Ulceration is rare.

GROUP II Focal macrophage granuloma formation, with central necrosis, surrounded by lymphocytes and plasma cells. Initially moderate numbers of organisms are present in surviving macrophages but later these disappear. Ulceration may occur independently of the necrotic granuloma.
\end{abstract}

GROUP III The most non-specific group. A mixed inflammatory response consisting mainly of lymphocytes and plasma cells but with a few macrophages. Amastigotes scanty but are best seen in the subepidermal zone.

GROUP IV Giant cells of foreign body and Langhans types are present together with a heavy lymphocytic infiltrate. Scanty or absent amastigotes.

GROUP V Tuberculoid response. Epitheloid cell granulomas with many Langhans giant cells but small numbers of lymphocytes and plasma cells. No necrosis. No amastigotes.

Adapted from: Ridley DS, Pathology. In: Peters W, Killick-Kendrick R (Eds). The Leishmaniases in Biology and Medicine, vol. 2. London, Academic Press, 1987, p. 665-702.

These observations prompted Ridley ${ }^{4}$ to propose a histological spectrum for cutaneous leishmaniasis, rather analogous to that for leprosy, ranging from anergic forms of infection with heavily parasitised macrophages to hypersensitive forms with scanty organisms and a tuberculoid response. There are five groups arranged in order of descending parasite load and these include the whole spectum of CL from DCL to recidivans (Table 2). However, whereas in leprosy there is a straight line spectrum from anergy to hypersensitivity, in individual cases of CL there are aberrations in which the parasite load is out of step with the host cell response.

\title{
IMMUNOLOGY
}

The development of longstanding resistance to reinfection is the principal immunological feature of classical CL. Reinfection amongst residents in regions for $L$. tropica is uncommon and experimental infections in human volunteers confirm that resistance develops after recovery provided the initial infection runs its full course. Immunity is not always complete; there are reports of reinfection occurring five or more years after initial cure or as a result of steroid therapy. In one case reinfection by the same L. major type followed spontaneous healing of the original lesion although there were fewer parasites and the lesion healed more rapidly than the primary infection. ${ }^{5}$

It is the cell-mediated immune response that provides resistance to infection with leishmania. ${ }^{6}$ Although antibodies to leishmania can be detected in the majority of patients with $\mathrm{CL}$, they are not protective even when present in high titre. ${ }^{3}$ The CMI response can be demonstrated clinically by the leishmanin skin test which is a delayed-type hypersensitivity reaction to the inoculation of a suspension of phenolised promastogotes (see diagnosis). Patients usually become leishmanin positive within a few weeks of infection and once positive remain so for decades. The significance of this long-lasting skin reactivity is unclear. It may represent genuinely long-lived immunological memory or it may be explained by 
TABLE 3

Cutaneous manifestations of leishmaniasis

\begin{tabular}{llc}
\hline \multicolumn{1}{c}{ Acute disease } & \multicolumn{1}{c}{ Chronic disease } & \multicolumn{1}{c}{ Miscellaneous } \\
\hline $\begin{array}{l}\text { Old World CL } \\
\text { wet/rural sore } \\
\text { dry/urban sore }\end{array}$ & non-healing acute sore & Leishmanioma \\
mucocutaneous leishmaniasis & Post Kala-Azar \\
New World (Američan) CL & liffuse cutaneous leishmaniasis recidivans & \\
\hline
\end{tabular}

the existence of an occult infection in recovered individuals. Nonetheless, this delayed hypersensitivity reaction does not represent a measure of resistance to reinfection. The reaction generally becomes positive early in the course of the disease whereas the lesion heals many months later. Reinoculation of an individual with both an active sore and a positive skin test leads to the development of a typical ulcer that develops to the same stage as the original sore and they then heal together. ${ }^{5}$

Leishmania infection in mice has been extensively studied as a model for a disease that is solely dependent on the CMI response for resolution. It was initially established that $\mathrm{CD}^{+} \mathrm{T}$ cells played an important role in the host's resistance to infection but, more recently, functional subsets of $\mathrm{T}$ cells known as TH-1 and TH-2 have been identified on the basis of their cytokine secretion profile. TH-1 cells, which are thought to mediate CMI, secrete IFN- $\alpha$ and IL-2 and are associated with resistance to infection. TH-2 cells, which are thought to be helper cells for specific antibody production, secrete IL-4 and IL-5 and are associated with susceptibility to infection. ${ }^{7}$ These observations were followed up by blocking IFN- $\alpha$ and IL-2 with monoclonal antibodies (mAbs) in infected mice: strains of mice genetically resistant to infection with Leishmania treated with anti-IFN- $\alpha \mathrm{mAb}$ developed progressive infections whilst treatment of susceptible strains with anti-IL-4 mAb attenuated the disease in all these mice and resulted in complete resolution of lesions in 85 per cent with the establishment of protective immunity. These findings opened the door for clinical trials of immunotherapy using human recombinant cytokines. In a controlled study, intralesional treatment with recombinant IFN- $\alpha$ produced a reduction in size of 12 out of 13 lesions due to L. braziliensis guyanesis after 3 weeks and some lesions were free of parasites. Similarly 9 out of 13 lesions due to L. tropica resolved completely with 4-8 weeks of treatment. Resolution was associated with Langerhan's cell hyperplasia, expression of HLA-DR by keratinocytes and increased numbers of $\mathrm{IL}_{-} 2^{+} \mathrm{T}$ cells indicating that the therapy with $\mathrm{r}-\mathrm{IFN}-\alpha$ promotes healing of CL by enhancing the CMI response. ${ }^{8.9}$

\section{CLINICAL FEATURES}

The clinical features of CL tend to differ between different regions of the world reflecting differences in the species of the parasite, the sandfly vector and the genetic, immunological and cultural background of the patients. ${ }^{1}$ The cutaneous manifestations of leishmaniasis are classified in Table 3. The lesions develop where the infected sandfly bites and are therefore usually on the exposed parts of the body. A cluster of lesions may occur and this has been attributed to repeated probings by the sandfly which has difficulty aspirating blood through its 
promastigote-laden proboscis. The incubation period is $1-12$ weeks tending to be shorter with a larger inoculum. ${ }^{3}$ Although each parasite is classically associated with a distinctive clinical picture, the features overlap and they cannot be reliably distinguished on clinical grounds alone.

Old world acute $C L$ (oriental sore). The lesion starts as an erythematous papule, rather like an insect bite with which it is frequently confused, but it persists and enlarges to form a painless nodule with a surface crust resembling a volcano. In some cases there is a larger subcutaneous component which has given rise to the term 'iceberg nodule'. In most cases the crust separates to expose an ulcer. ${ }^{10}$ Lesions heal by fibrosis leaving a sharply demarcated, irregular, cribriform scar which in coloured races is often hyperpigmented and disfiguring. Lesions, particularly in cases due to L. major, are often multiple and occasionally over 100 lesions occur in a single patient. ${ }^{3}$

Oriental sores are often divided into wet and dry types. The wet, rural or zoonotic, sore is a more inflammatory lesion which is classically associated with L. major and is usually acquired in rural areas where the infecting organisms are also rodent parasites and are poorly adapted to man. After an incubation period of less than two months an erythematous papule develops and two weeks later a central crust appears which usually falls off revealing a bright red base consisting of slough alternating with papules of proliferating granulation tissue. The ulcer enlarges over the following two to three months reaching a diameter of $3-6 \mathrm{~cm}$. Multiple satellite papules which measure $2-5 \mathrm{~mm}$ and do not contain parasites are much more common in the wet than in the dry type. The lesion heals in three to twelve months.

The dry, urban or anthroponetic, sore is a more quiescent but indolent lesion. It is classically associated with L. tropica and is more commonly found in urban areas where the parasite is better adapted to man. The lesion starts as a brownish nodule which enlarges to form a plaque measuring $1-2 \mathrm{~cm}$ in diameter in about six months. Ulceration then appears in the centre associated with a closely adherent crust. Healing may take up to two years.

Lesions due to $L$. aethiopica are usually single and most commonly occur on the central part of the face. Satellite papules amalgamate into a large spreading nodule. Ulceration or crusting is not always a feature. There is relatively little inflammation and the lesions heal over two to five years. It may progress to DCL. L. infantum, which generally causes visceral leishmaniasis in children, usually gives rise to a self-healing sore in adults. Its appearance and evolution are slow compared to lesions due to $L$. major ${ }^{1.10 .11,12}$

New World CL. Infection due to either $L$. mexicana or $L$. braziliensis complex produces localised nodulo-ulcerative disease which is similar in many respects to that seen in Old World disease. The lesion starts as an erythematous papule which forms a crust then rapidly ulcerates. The ulcer base is necrotic and often secondarily infected with either Staphylococus aureus or $\beta$-haemolytic Streptococcus. It is surrounded by an indurated violaceous border. In an analysis of 187 British soldiers with parasitologically proven cutaneous leishmaniasis contracted in Belize, most lesions occurred on exposed body sites (Fig 2). Lesions due to L. mexicana mexicana were more common on the head and neck compared to those due to $L$. 


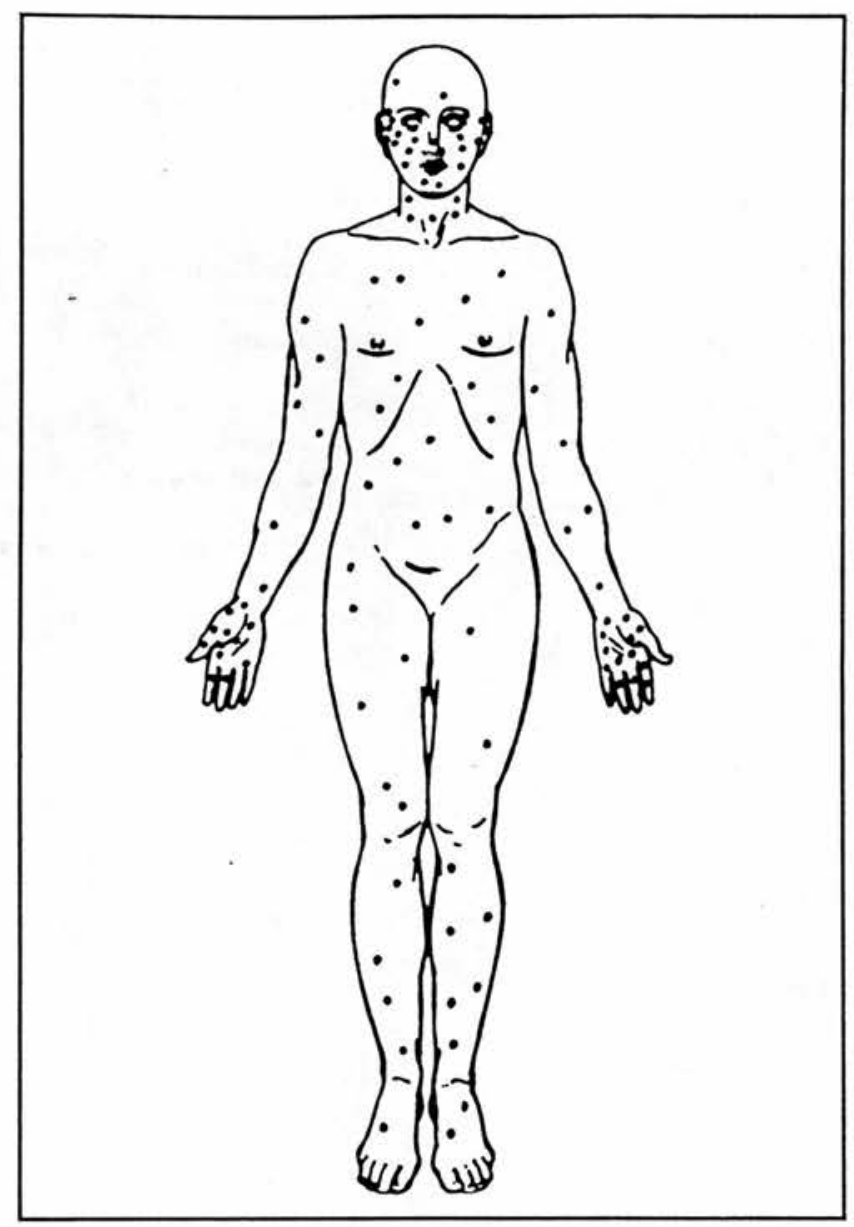

FIGURE 2

Site of lesions in 187 parasitological proven cases of cutaneous leishmaniasis from Belize (courtesy of $J$ Derm Treat).

braziliensis braziliensis. A single lesion was present in 71 per cent of cases and only one per cent had more than 4 lesions. The average diameter of the lesions at diagnosis was $14.4 \mathrm{~mm}$ (SD 9.3). It was not possible to distinguish lesions due to $L$. mexicana mexicana from those due to $L$. braziliensis braziliensis on the basis of their clinical appearance alone. ${ }^{14}$

Lesions due to $L$. mexicana complex usually heal spontaneously within six to eight months although those occurring on the pinna (chiclero's ulcer) often persist and cause extensive local destruction. ${ }^{12}$ L. m. amazonensis is associated with DCL. L. braziliensis complex causes localised disease which may eventually heal spontaneously but it is associated with the destructive mucocutaneous form of the disease known as 'Espundia'. This usually starts in the nasal mucosa but progressively destroys the nose, upper lip, palate, pharynx and larynx leading to death from secondary infection, starvation or laryngeal obstruction. It has been attributed to metastatic spread to the nasopharyngeal mucosa and may present within 3 years of the primary lesion but it is often delayed for over 20 years. ${ }^{13}$ 
TABLE 4

Differential diagnosis of cutaneous leishmaniasis.

\begin{tabular}{ll}
\hline Acute & Chronic \\
Insect bite & \\
Trauma & Keloid \\
Foreign body granuloma & \\
Pyogenic granuloma & Lupus vulgaris \\
Leprosy & \\
Tropical ulcer & Syphilitic gummata \\
Impetigo & \\
Furuncle & \\
Carbuncle & Sarcoidosis \\
Ecthyma & Granuloma faciale \\
Orf & Discoid lupus erythematosus \\
Tularaemia & Jessner's lymphocytic infiltrate \\
Milker's nodule & Lymphoma \\
Swimming pool granuloma & Wegener's granulomatosis \\
Syphilitic granuloma & \\
Yaws & \\
Sporotrichosis & \\
Blastomycosis & \\
Kerion & \\
Mollusca contagiosum & \\
Viral warts & \\
Myiasis & \\
Dracunculosis & \\
Keratoacanthoma & \\
Basal cell carcinoma & \\
Squamous cell carcinoma & Metastases \\
Lymphoma & \\
Leukaemia & \\
\hline
\end{tabular}

(Adapted from Kubba et al. 1987).

\section{DIAGNOSIS}

This is frequently made on the basis of the clinical appearance of the lesion together with a history of exposure to infection. However, the differential diagnosis is extensive (Table 4) and it is therefore important to seek pathological confirmation, preferably by demonstration of the parasite either morphologically or by culture. Organisms are most numerous in the active edge of the lesionstherefore samples should be taken from that area.

Skin biopsy. A wedge or punch biopsy, under local anaesthetic, can be used for the preparation of impression smears, parasite culture and routine histological examination. The area should be thoroughly cleaned with $6 \%$ hydrogen peroxide to reduce bacterial contamination prior to biopsy. Amastigotes (L-D bodies) can be identified in routine $\mathrm{H} \& \mathrm{E}$ sections during the early stages of the disease but they become less numerous with the passage of time and may be impossible to find after a few months. The typical granulomatous histology is a much less specific diagnostic pointer. In these circumstances it may be possible to detect amastigotes using polyclonal antibodies in conjunction with immunoperoxidase 
staining. ${ }^{15}$ Monoclonal antibody probes in conjunction with immunofluorescent assays are currently being developed ${ }^{16}$ but are not generally available.

Smear preparations. Can be prepared by pressing a skin biopsy against a glass slide prior to fixation or by using tissue juice obtained either by aspirating the edge of the lesion with a 20 gauge needle or by using a slit-skin technique akin to that used in leprosy. Amastigotes may be detected both intra- and extracellularly. ${ }^{17}$.

Culture of leishmania is often difficult and involves both art and science. Whilst some species, such as $L$. mexicana mexicana, grow readily others, such as $L$. braziliensis braziliensis, are more difficult. Essentially the tissue biopsy is placed in a biphasic blood-agar medium such as a modified NNN to which antibiotics are added to prevent bacterial overgrowth and incubated at $22^{\circ} \mathrm{C}$. Promastigotes may be identified in as little as seven days although it often takes up to 21 days. The techniques for culture (and also isoenzyme typing) are clearly described in an handbook by Evans. ${ }^{18}$ Animal inoculation is a useful alternative to in vitro culture, particularly when the parasite load is low or if sterile conditions are difficult to achieve. The golden hamster is generally the most suitable as it is sensitive to most strains of leishmania, although the $\mathrm{Balb} / \mathrm{c}$ mouse is best for $L$. major. Homogenised tissue containing amastigotes should be injected intradermally into the nose, ears or footpads and the inoculation site biopsied seven to ten days later to obtain samples for culture or at a later date for histological examination. ${ }^{19}$

Identification of Leishmania species. Isoenzyme analysis remains the method of choice for typing leishmania because it is simple to use, reproducible and well established. It represents the standard against which the newer methods, such as monoclonal antibodies and DNA hybridization probes, are assessed. An aqueous extract containing many enzymes is prepared from promastigotes cultured from the lesion. This extract is analysed by electrophoresis during which the banding pattern, identified by staining for a single enzyme such as glucose 6 phosphate dehydrogenase or malic dehydrogenase, is compared with that of a known reference strain of leishmania. Several such zymogrammes, each stained for a different enzyme, are required to identify a given species. ${ }^{18}$

DNA hybridization probes for kinetoplast DNA are being developed which ailow identification of cultured promastigotes or amastigotes from lesions. The technique is very sensitive-identification is possible with as few as 50 organisms-but there are problems with non-specific labelling. These techniques are well described in a manual by Barker. ${ }^{20}$ As these techniques improve and as more probes become available these methods will probably replace isoenzyme analysis as the routine diagnostic tool.

Serological diagnosis. Serological methods are widely used in the diagnosis of visceral leishmaniasis and antibodies are also present in cutaneous disease albeit in low titre. However, serology has been insufficiently sensitive and specific to be helpful diagnostically in Old World disease ${ }^{1}$ and also, in our hands at least, in New World disease.

Leishmania (Montenegro) skin test. This is a delayed hypersensitivity reaction, 
resembling the tuberculin test, in which $0.1 \mathrm{ml}$ of a suspension containing $10^{5}$ promastigotes in 0.5 per cent phenol saline is injected intradermally into the forearm. An equal amount of 0.5 per cent phenol saline alone is injected into a second, adjacent, site as a control to determine sensitivity to the diluent. An indurated nodule with surrounding erythema develops and the reaction is read at $48-72 \mathrm{~h}$. An area of induration measuring $5 \mathrm{~mm}$ or more in diameter is positive. Unfortunately, because standardised test antigens are not available the reagents are produced in the laboratories of tropical disease centres and vary in their sensitivity and specificity. ${ }^{21}$ Its diagnostic utility is limited because, although over 90 per cent of patients with CL give a positive reaction, so do up to 9 per cent of healthy controls in endemic areas presumably from past, often asymptomatic, infection.

\section{TREATMENT}

The optimal management of CL has not yet been determined. The literature abounds with reports of 'effective' treatments. However, given the self-healing nature of most of the lesions together with the distinct lack of controlled therapeutic trials, selection of the most appropriate therapy, if any, can be difficult. There are, however, several guiding principles. Lesions due to different species of leishmania differ in severity and in their response to treatment; in general New World forms tend to be more severe and longer lasting than Old World forms.

In the Old World the vast majority of patients have lesions which will heal spontaneously within a few months or at most two years. Active treatment is therefore not mandatory-indeed for patients living in endemic areas it may not be appropriate because spontaneous healing is associated with the development of protective immunity. However, patients with lesions on cosmetically or functionally important sites, such as the face or hands, those with associated lymphangitis and those with multiple lesions, are best given active treatment. Local treatment is appropriate for early non-inflamed lesions and systemic therapy for those with multiple or more complicated lesions. An exception to this rule are those lesions due to $L$. aethiopica which is relatively resistant to antimony and for which local treatments have not been validated. The WHO recommends that these lesions are left to heal spontaneously unless they occur in the oronasal area, in which case, systemic pentamidine should be used. ${ }^{1}$

In New World disease the first crucial step is to decide whether or not one of the $L$. braziliensis complex could be the infecting organism because, until further information is available, all such cases must be considered capable of causing mucocutaneous disease and therefore adequate systemic therapy is mandatory. Hence, if the patient contracted CL in any area in which the L. braziliensis complex occurs (i.e. Belize, Honduras, Costa Rica, Panama, Venezuela, Colombia, Suriname, French Guiana, Brazil, the western slopes of the Peruvian Andes and the Argentinian Highlands, ${ }^{22}$ the parasite must be isolated and identified before any alternative to optimal systemic treatment can be considered. If the disease was contracted in other areas, or if the disease is shown to be due to the L. mexicana complex (except L. mexicana amazonensis), alternatives, including topical or no treatment at all, may be considered. The WHO recommends that 
L. mexicana amazonensis is treated in the same way as L. braziliensis complex lesions because a high proportion develop DCL.

Pentavalent antimonials remain the mainstay of effective systemic treatment for all forms of leishmaniasis because of their undoubted efficacy and, when properly used, safety. Two products are available: sodium stibogluconate (Pentostam; Burroughs-Wellcome, UK) and meglumine antimoniate (Glucantime; Specia, France). They are similar in both efficacy and toxicity-any reported differences can be explained by their differing antimony concentrations (Pentostam: $100 \mathrm{mg} / \mathrm{ml}$; Glucantime: $85 \mathrm{mg} / \mathrm{ml}$ ). Antimonials decrease the availabilty of adenosine triphosphate (ATP) and guanosine triphosphate (GTP) through inhibition of glycolysis and the citric acid cycle. ${ }^{2}$ Both drugs must be administered parenterally, either by slow i.v. or i.m. injection. They are rapidly excreted, unchanged, by the kidney with a half-life of less than six hours. They should be administered in a dose of $10-20 \mathrm{mg} / \mathrm{kg}$ body weight daily in two equal doses for two to four weeks or until clinical and parasitological cure is achieved. In the case of lesions due to $L$. braziliensis complex, the WHO recommends $20 \mathrm{mg} / \mathrm{kg} /$ day for a minimum of 4 weeks. Serious toxicity is rare although patients often report malaise, anorexia, myalgia and arthralgia during treatment. An elevation in serum transaminases, a mild leucopenia and flattening of the $\mathrm{T}$ waves on the ECG are common during therapy and appear dose related. These abnormalities settle rapidly when treatment is stopped.

Pentamidine is a second-line drug except in cases due to $L$. aethiopica. It acts against leishmania by damaging the kinetoplast DNA-mitochondrial complex. It must be administered parenterally, either by i.m. or slow i.v. injection in a dose of $3-4 \mathrm{mg} / \mathrm{kg}$ body weight once or twice weekly until the lesion resolves. ${ }^{1,2.12}$ The main adverse effects are hypoglycaemia, myoglobinuria with nephrotoxicity and cardiotoxicity. It has a narrow therapeutic index and it is therefore generally preferable to give doses less frequently but for a longer period. ${ }^{23}$

Ketoconazole has been used in recent years to treat Old World CL although its efficacy has not been formally evaluated in controlled trials. It is administered orally in a dose of $600 \mathrm{mg}$ daily for 28 days and the lesion should be re-assessed once a month after treatment. ${ }^{24}$

Local treatments for CL include physical modalities such as surgical excision, curettage followed by cautery, cryotherapy and local heat treatment. ${ }^{17}$ Local infiltration of lesions with sodium stibogluconate in a dose of $1-3 \mathrm{ml}$, repeated once or twice at 1-2 day intervals, is a convenient treatment for early noninflamed lesions. Technique is important-infiltration must produce complete blanching at the base of the lesion. ${ }^{1}$ An exciting recent development has been the development of an ointment containing 15 per cent paromomycin and 12 per cent methyl benzethoniuim chloride in white soft paraffin which is applied twice daily for 10 days. This regimen has been reported to cure 87 per cent of patients with Old World disease in 10 to 30 days. ${ }^{25}$ Unfortunately, this formulation is very irritant and alternative formulations have been less effective. ${ }^{26}$

In conclusion, CL remains a major world health problem, but its importance is now being recognised and research efforts are being directed towards improved diagnosis and treatment. To date, progress has been evolutionary rather than revolutionary. 


\section{REFERENCES}

${ }^{1}$ WHO Expert Committee. Control of leishmaniasis. Technical Report Series 793. Geneva: WHO 1990.

${ }^{2}$ Kubba R, Al-Gindan Y. Leishmaniasis. Derm Clin 1989; 7: 331-51.

${ }^{3}$ Kalter DC. Cutaneous and mucocutaneous leishmaniasis. Prog Derm 1989; 23: 1-11.

${ }^{4}$ Ridley DS. Pathology. In: Peters W, Killick-Kendrick R. Eds. The leishmaniases in biology and medicine. London: Academic Press 1987; 665-729.

${ }^{5}$ Mauel J, Behin R. Immunity: clinical and experimental. In: Peters W, Killick-Kendrick R. Eds. The leishmaniases in biology and medicine. London: Academic Press 1987; 731-91.

${ }^{6}$ Liew FY, Immunity of protozoa. Current opinion in immunology 1989; 1: 441-47.

${ }^{7}$ Miller LH, Scott P. Immunity to protozoa. Current opinion in immunology 1990; 2: 368-74.

${ }^{8} \mathrm{Gran}$ GE, Modlin RL. Immune mechanisms in bacterial and parasitic diseases: protective immunity versus pathology. Current opinion in immunology 1991; 3: 480-5.

${ }^{9}$ Harms G, Zwingenberger K, Chéhade AK et al. Effects of intradermal gamma-interferon in cutaneous leishmaniasis. Lancet 1989; i: 1287-92.

${ }^{10} \mathrm{Kuba}$ R, Al-Gindan Y, El-Hassan AM, Omer AHS. Clinical diagnosis of cutaneous leishmaniasis (oriental sore). J Am Acad Derm 1987; 16: 1183-9.

${ }^{11}$ Griffiths WAD. Old World cutaneous leishmaniasis. In: Peters W, Killick-Kendrick R. Eds. The leishmaniases in biology and medicine. London: Academic Press 1987; 617-36.

${ }^{12}$ Bryceson ADM. Leishmaniasis. In: Champion RH, Burton JL, Ebling FJG. Textbook of dermatology 5 Ed. London: Blackwell Scientific 1992; 1251-63.

${ }^{13}$ Walton BC. American cutaneous and mucocutaneous leishmaniasis. In: Peters W, KillickKendrick R Eds. The leishmaniases in biology and medicine. Academic Press, London 1987; 637-64.

${ }^{14}$ Hepburn NC. The management of American cutaneous leishmaniasis. J Derm Treat 1992; 3: 95-8.

${ }^{15}$ Sells PG, Burton M. Identification of leishmania amastigotes and their antigens in formalin fixedtissue by immunoperoxidase staining. Trans $R$ Soc Trop Med Hyg 1981; 75: 461-8.

${ }^{16}$ Anthony RL, Grogl M, Sacci JB, Ballou RW. Rapid detection of leishmania amastigotes in fluid aspirates and biopsies of human tissue. Am J Trop Med Hyg 1987; 37: 271-6.

${ }^{17}$ Hepburn NC, Omer AHS. Old World cutaneous leishmaniasis. In: Handbook of infectious diseases. Philadelphia: Decker (in press).

${ }^{18}$ Evans D. Handbook on isolation, characterisation and cryopreservation of leishmania. Geneva: UNDP/World Bank/WHO (TDR), 1989.

${ }^{19}$ Manson-Bahr PEC. Diagnosis. In: Peters W, Killick-Kendrick R. Eds. The leishmaniases in biology and medicine. London, Academic Press; 1987: 703-29.

${ }^{20}$ Barker DC, Butcher J, Gibson LJ, Williams RH. Characterisation of leishmania Sp. by DNA hybridization probes-a laboratory manual. Geneva: UNDP/World Bank/WHO (TDR), 1986.

21 Weigle KA, Valderrama L, Arias AL et al. Leishmanin skin test standardisation and evaluation of safety, dose, storage, longevity of reaction and sensitisation, Am J Trop Med Hyg 1991; 44: $260-71$.

${ }^{22}$ Lainson R, Shaw JJ. Evolution, classification and geographical distribution. In: Peters W, KillickKendrick R Eds. The leishmaniases in biology and medicine. London: Academic Press 1987: $1-120$.

${ }^{23}$ Bryceson A. Therapy in man. In: Peters W, Killick-Kendrick R Eds. The leishmaniases in biology and medicine. London: Academic Press 1987: 847-907.

${ }^{24}$ Norton SA, Frankenburg S, Klaus SN. Cutaneous leishmaniasis acquired during military service in the Middle East. Arch Dermatol 1992; 128: 83-7.

${ }^{25} \mathrm{El}-\mathrm{On} \mathrm{J}$, Livishin R, Evan-Paz Z et al. Topical treatment of cutaneous leishmaniasis. $J$ Invest Dermatol 1986; 87: 284-8.

${ }^{26}$ El-Safi SM, Murphy AG, Bryceson ADM, Neal RA. A double-blind clinical trial of the treatment of cutaneous leishmaniasis with paromomycin ointment. Trans $R$ Soc Trop Med Hyg 1990; 84: 690-1. 
${ }^{10}$ Hung J, Whitford EG, Parsons RW, Hillman DR. Association of sleep apnoea with myocardial infarction in men. Lancet 1990; 336: 261-64.

"Koskenvuo M, Kaprio J, Telakivi T et al. Snoring as a risk factor for ischaemic heart disease and stroke in men. Brit Med J 1987; 294: 16-19.

${ }^{12}$ Partinen M, Palomaki H. Snoring and cerebral infarction. Lancet 1985; 2: 1325-6.

${ }^{13}$ Spriggs DA, French JM, Murde JM et al. Snoring increases the risk of stroke and adversely affects prognosis. Q J Med 1992; 83: 555-62.

${ }^{14}$ Partinen M, Jamieson A, Guilleminault C. Long-term outcome for obstructive sleep apnea syndrome patients: Mortality. Chest 1988; 94: 1200-4.

${ }^{15}$ Crocker BD, Olson LG, Saunders NA et al. Estimation of the probability of disturbed breathing during sleep before a sleep study. Am Rev Respir Dis 1990; 142: 14-8.

${ }^{16}$ Douglas NJ, Thomas S, Jan MA. Clinical value of polysomnography. Lancet 1992; 339: 347-50.

${ }^{17}$ Douglas NJ, Calverley PMA, Leggett RJE, Brash HM, Flenley DC, Brezinova V. Transient hypoxaemia during sleep in chronic bronchitis and emphysema. Lancet 1979; 1: 1-4.

${ }^{18}$ Broughton RJ. Narcolepsy. In: Handbook of Sleep Disorders. Thorpy M. J. ed. New York: Marcel Dekker 1990, 197-216.

${ }^{19} \mathrm{He} \mathrm{J}$, Kryger $\mathrm{MH}$, Zorick FJ et al. Mortality and apnea index in obstructive sleep apnea: Experience in 385 male patients. Chest 1988; 94: 9-14.

${ }^{20}$ Chaban R, Cole P, Hoffstein V. Site of upper airway obstruction with idiopathic obstruction sleep apnea. Laryorgoscope 1988; 98: 641-7. 


\title{
CUTANEOUS LEISHMANIASIS *
}

\section{Part II: Historical Aspects, Epidemiology and Prevention}

\author{
N. C. Hepburn, Department of Dermatology, Royal Infirmary of Edinburgh
}

\section{HISTORICAL ASPECTS}

The leishmaniases are ancient diseases of man. In the Old World, descriptions of Balkh sore (named after Balkh, a town in the north of Afghanistan near the Russian border), date back to the first century AD. The disease has been traced back through the medieval literature of the Middle East by Elgood ${ }^{1}$ who speculates that it was carried from Balkh to Baghdad by the invading Mongols in $1215 \mathrm{AD}$ and that it then spread down to the Persian Gulf and, later, back along the trade routes into the interior of Iran reaching Tehran by 1840 . The disease has many synonyms, mostly derived from place names, such as Dehli boil, Aleppo boil, Armenian sore, Sart sore and the still commonly used oriental sore. ${ }^{2}$

In the New World, early records of cutaneous leishmaniasis are more difficult to identify because other endemic conditions, such as syphilis and blastomycosis, cause similar lesions. However, mucocutaneous leishmaniasis (espundia) can be traced back to pre-Columban times through anthropomorphic vessels, huacos, which have been found amongst the ancient Peruvian Mochica pottery in Inca tombs (c. 400-900 AD). Some of these figures depict the typical facial lesions as do terracotta figures from northern Equador. Shortly after the Spanish conquest, historians such as Pizarro (1517) noted a rare but decimating disease among the Peruvian Indians which primarily affected the nasal cavities. ${ }^{2}$ Gade has reviewed these classical descriptions of the disease which occurred in the same areas as it does today:

Indians are taken from the highlands to work in the coca fields in the 'Andes of Curzo' where they get an infection in the nostrils which also makes their nose trunk-like and red, from which some die.

... and the Indians get sick with an incurable disease called 'of the Andes', which is worse than syphilis... it attacks most commonly the nostrils, lips and uvula of the throat ... others get it in the mouth, making a sight one cannot look at without great horror and fright at such ugliness. ${ }^{3}$

In addition Spaniards who explored the high Andean valleys, where sandflies were common, occasionally developed mutilitating lesions of the nares similar to those depicted on the huacos. This disease was known as 'anti-onccoy' or 'the disease of the Andes' but only comparatively recently has the name for the long suspected cause, a small biting fly or uta, being applied to the disease itself. ${ }^{2}$ Names for the disease in its various forms are also profuse in the New World and include uta, espundia, mucocutaneous leishmaniasis, ulcera des los chicleros, boton de Baura, bouba, bay sore, forest yaws, pain bois and leishmaniose tegumentar americana.

The scientific study of leishmaniasis began with the publication in 1885 in Scientific Memoirs by Medical Officers of the Army of India by Surgeon-Major D. D. Cunningham of a description of amastigotes in sections of human skin

^Part I: Clinical features, pathology and treatment is in Proc R Coll Physicans Edinb 1992; 22: 448-59. 
from a patient with Dehli boil. ${ }^{4} \mathrm{He}$ described some 'deeply staining bodies in certain cells larger than lymphocytes' and attributed these to the spores of a slime mould. These observations were confirmed six years later by Firth. ${ }^{5}$

In 1898 Borovsky, a Russian military surgeon studying Sart sore in Tashkent, described 'small spherical, oval or fungal form corpuscles' measuring 1.5-2.0 $\mu \mathrm{m}$ diameter with a nucleus and a 'process running to the periphery'. Unlike Cunningham, he realised these were protozoa and the cause of the disease. Unfortunately his observations were published in an obscure Russian journal 'Voenno-Medicinskij-Zürnal' and the significance of his discovery was not recognised for many years. ${ }^{6}$

In 1903 the pace of discovery quickened, Leishman (1865-1926) a British army pathologist, reported the similarity of degenerating trypanosomes from the spleen of a laboratory rat which had died after an experimental infection with African trypanosomes 48 hours earlier to bodies he had observed some three years previously during a post morten examination of the splenic pulp of a soldier 36 hours after death from a tropical cachexia. The soldier had contracted his illness during service at Dum-Dum seven miles from Calcutta. ${ }^{7}$ Leishman concluded that these organisms had been degenerating trypanosomes. Although incorrect, this was the first description of a possible causative agent for visceral leishmaniasis. Subsequently Donovan, a Captain in the Indian Medical Service at Madras, described similar organisms in a fresh biopsy from the spleen of a patient with visceral disease revealing it was a different organism rather than a degenerate trypanosome. $^{8}$ Later that year Wright, whilst working in Boston, USA, and using a greatly improved Romanowsky stain, first accurately described and photographed the cutaneous Old World parasite obtained from the cheek of a nine year old girl who had developed the lesion two or three months earlier whilst in Armenia. ${ }^{9}$

In 1904 Rogers successfully cultured the visceral parasite by incubating cultures at $22^{\circ} \mathrm{C}$ and in 1908 Nicolle cultured the cutaneous organism using a modification of the rabbit blood nutrient agar medium of Novy and McNeal known as NNN medium. ${ }^{2}$

The first report of the New World organism was made in 1909 by Lindenburg who had been examining ulcers on the face and forearms of railway workers in São Paulo. The clinical features of espundia, a leishmanial infection involving erosion of the palate, were first described and illustrated by Carini (1911) whilst working at the Pasteur Institute in São Paulo. In the same year Vianna described parasites present in ulcers contracted in Minas Gerias, Brazil; he was able to discern a nucleus, kinetoplast and a 'rudimentary flagellum' and proposed the name Leishmania braziliensis to distinguish it as the cause of espundia. ${ }^{2}$ In 1913 an expedition from Harvard investigated and succeeded in discovering, independently, the cause of uta in Peruvian mountain towns. They described and illustrated the parasites together with the clinical features including erosion of the palate. They were also able to culture the organism on blood agar and reported that it was believed to be transmitted by the genus Phlebotomus. ${ }^{10}$

The sandfly has been implicated as the vector of cutaneous leishmaniasis as far back as $1764 .{ }^{11}$ In 1904 Rogers observed the conversion of amastigotes to promastigotes in culture ${ }^{12}$ and subsequently promastigotes were found in sandflies by Alder and Theodor in 1925. ${ }^{13}$ In 1921 a volunteer developed a cutaneous lesion after inoculation with a suspension of caught Phlebotomus ${ }^{14}$ and then in 


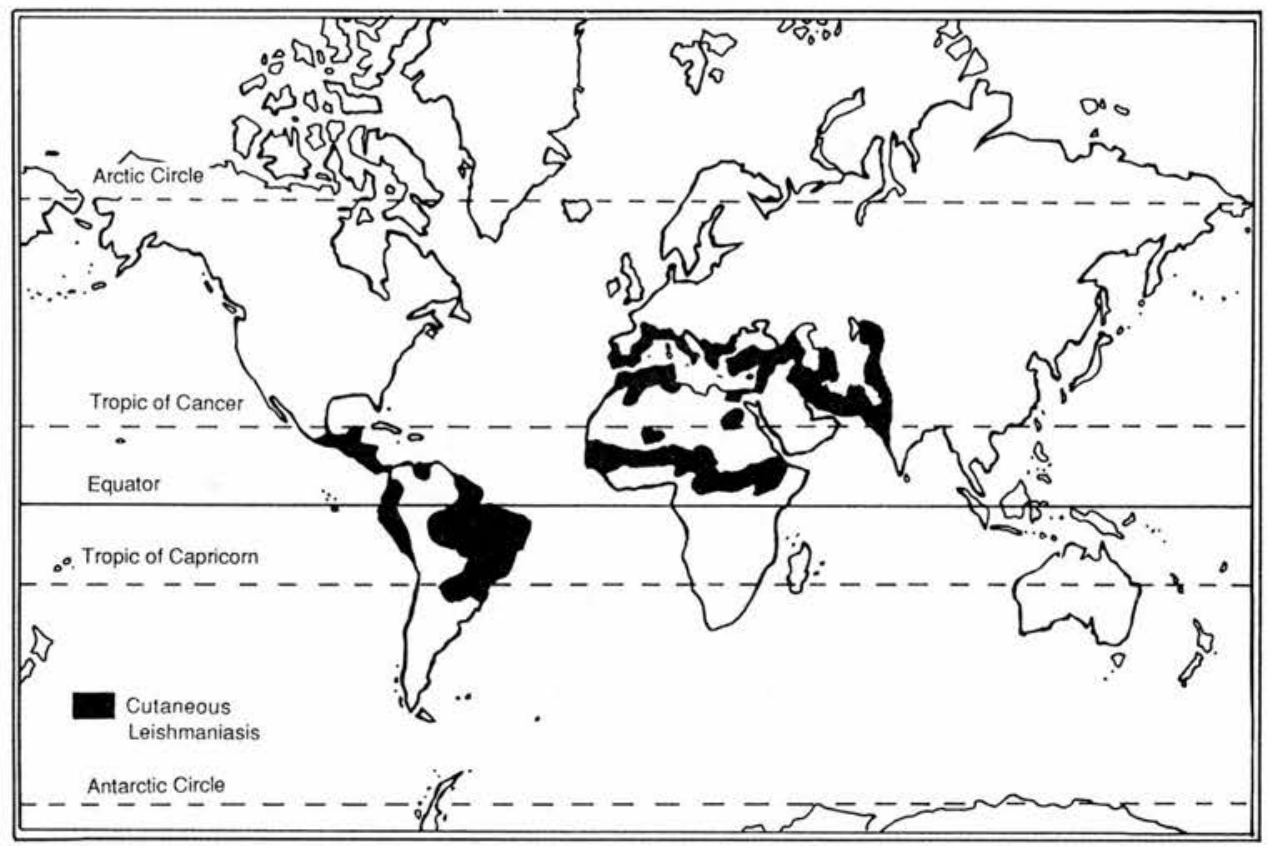

FIGURE 1

Geographical distribution of cutaneous leishmaniasis.

1931 Shortt was able to transmit L.donovani to hamsters by the bite of Phlebotomus argentypes. ${ }^{15}$ It was not until 1941, however, that L.tropica was transmitted to volunteers by the bite of experimentally infected Phlebotomus papatasi $^{14}$ thus confirming the cycle.

\section{EPIDEMIOLOGY}

Research during the past decade has revealed that the leishmaniases-both visceral and cutaneous - are much more prevalent than had been appreciated. In 1990 WHO estimated that 12 million people were affected in over 80 countries and that 350 million people were at risk of infection. ${ }^{17}$ More specifically, Ashford et al. ${ }^{18}$ estimate that approximately 300,000 cases of cutaneous leishmaniasis occur each year in 61 countries; they also estimate that 185 million people are at risk of cutaneous disease. Furthermore, there is good evidence for a dramatic increase in incidence in the New World. ${ }^{19}$ The geographical distribution of cutaneous leishmaniasis is shown in Figure 1. However, it is important to note that more than half the cases occur in just eight countries: Afghanistan, Algeria, Brazil, Iran, Iraq, Saudi Arabia, Sudan and Syria.

The cycle of infection. The leishmaniases are complex protozoal infections transmitted by the bite of an infected female sandfly (Phlebotomus in the Old World, Lutzomyia or Psychodopygus in the New World). In most cases these infections exist as zoonoses amongst wild animals and man is only infected as an incidental host (Figure 2). However, such infections are by no means uncommon in 


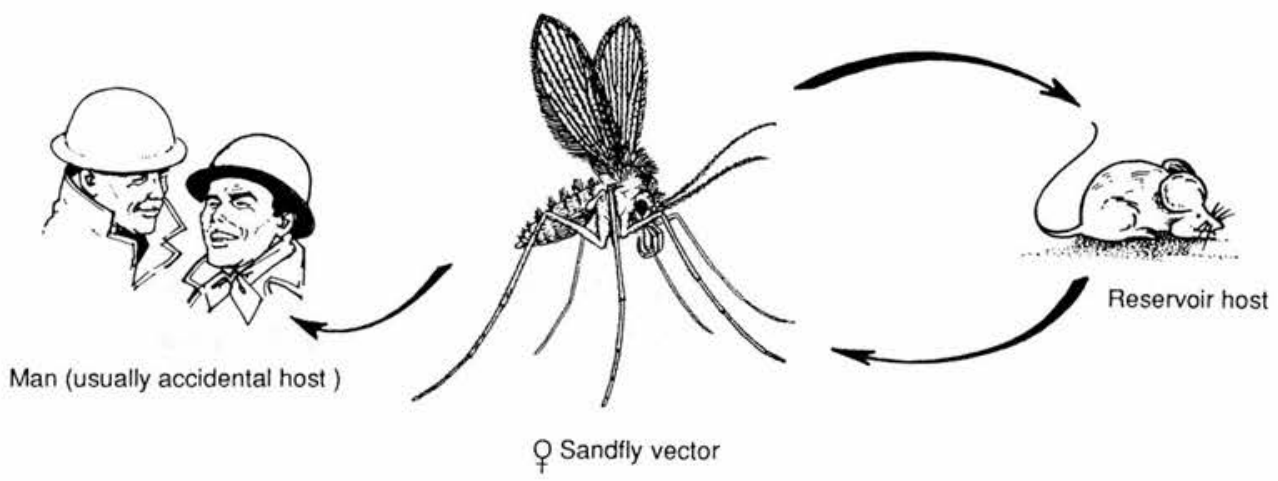

FIGURE 2

The cycle of infection.

endemic areas where up to 9 per cent of the healthy population may have a positive leishmanin skin test indicative of an earlier, often asymptomatic, infection. ${ }^{20}$ The requirements for vector species, reservoir host and environment tend to be very specific for each leishmanial species, consequently the disease is often localised to small areas where all three exist together (Table 1).

Vector biology. Sandflies are extremely small, measuring less than $3.5 \mathrm{~mm}$, they are covered with a sandy yellow or brown coat of hair and have very dark eyes. The male has prominent terminal organs for grasping the female. These tiny flies live in dark, damp places and make characteristic little hops when disturbed. They are able to fly remarkably well and, although the usual radius is only $50 \mathrm{~m}$ from the breeding site, ranges up to $1-2 \mathrm{~km}$ have been recorded. ${ }^{21.22}$ Only females are blood sucking as blood is essential for egg development. They bite mainly at dusk, their approach is silent, unlike that of most mosquitos, and the exposed parts are usually attacked. Their minute eggs are laid in cracks and crevices in dark, moist sites. They hatch in 9-12 days and go through 4 larval stages followed by a pupal stage before finally developing into a fly. Under favourable conditions the life-cycle takes about one month but it may be considerably prolonged under adverse circumstances. ${ }^{21}$ Temperature is the only factor that correlates well with sandfly distribution in the world. Although sandflies are able to survive cold temperatures in diapose (a period of suspended development accompanied by greatly decreased metabolism), larval development and adult activity are greatly reduced at temperatures below $20^{\circ} \mathrm{C}$. Their distribution is therefore largely confined to areas that have at least one month with a mean temperature of $20^{\circ} \mathrm{C} .^{23}$

As sandflies lacerate the skin to feed upon a pool of serosanguinous exudate, they ingest amastigotes which develop into promastigotes in the gut before migrating to the salivary glands after seven to ten days, after which time infective promastigotes can be regurgitated via the proboscis during the next blood meal. During feeding saliva is introduced into the host and it has recently been discovered that lysates of the salivary glands from Lutzomyia longipalpis greatly enhance the infectivity of L. major in mice. It has long been known that a sandfly 


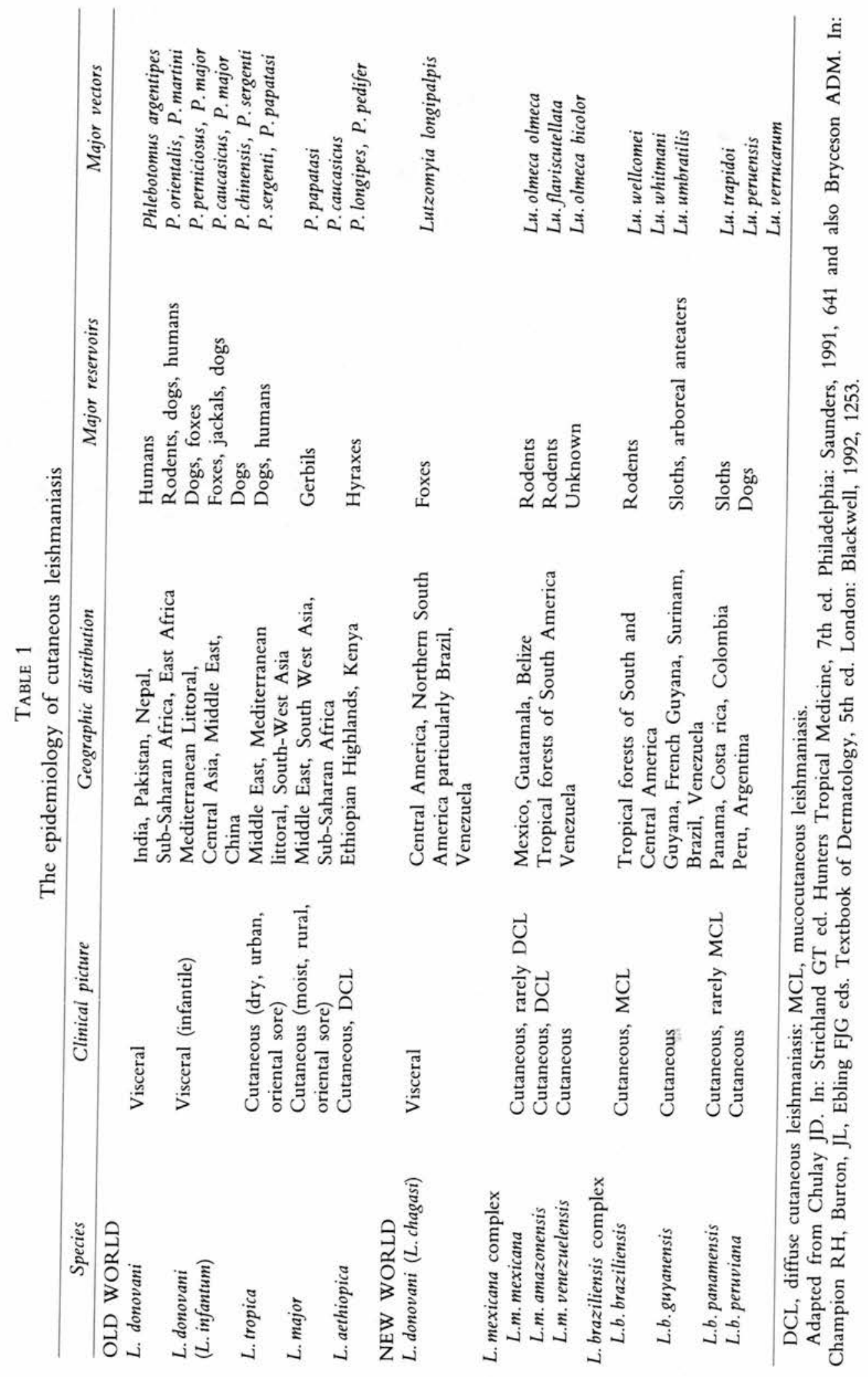


injects about $10-100$ parasites during a blood meal. However, when a mouse is inoculated with this number of parasites none survive unless sandfly saliva is also injected-approximately $10^{5}-10^{6}$ parasites are needed for laboratory infection. ${ }^{24}$ In addition, if salivary gland lysate is present lesions develop and grow more rapidly. These lysates have been analysed and a very potent vasodilator, maxadilan, which is similar to the mammalian neuropeptide calcitonin gene related peptide, has been isolated. This is able to inhibit the production of hydrogen peroxide by macrophages in response to interferon gamma-the mechanism by which hosts destroy intracellular parasites. ${ }^{25}$

The biology of each sandfly species is unique and complex, and it is beyond the scope of this article to describe each one. However, before a particular species can be implicated as the vector for a given type of leishmania, it must be established that the fly bites both man and the reservoir host, that both are infected in nature with the same type of leishmania, that the fly supports a flourishing growth of the parasite and it is able to transmit it by biting, and finally that the geographical distribution of the fly and parasite are similar. ${ }^{17}$ In many cases our knowledge is incomplete but the overall picture is summarised in Table 1.

Reservoir hosts. Leishmania spp are maintained indefinitely within an ecological system consisting of one or a small number of sandfly vectors and one or a few vertebrate reservoir hosts. There is usually one primary reservoir host for a given species within a particular focus but other animals in the same area may become infected forming secondary hosts (from which leishmania can be transmitted to other mammals) or incidental hosts (from which leishmania cannot be passed on). These hosts may not show obvious signs of infection-often there are relatively few amastigotes in the skin or viscera and the host immune response is minimal. By way of contrast, dogs, the natural reservoir for L.donovani which causes visceral disease in man, may eventually be killed by the infection. The parasites are abundant in both the viscera and the dermis from which they are readily picked up by the sandfly vectors: thus the site of leishmania in the host does not necessarily correspond to the site of disease in man. A further example is that of L. guyanensis which infects the internal organs of the reservoir sloth with minimal skin involvement but produces cutaneous disease in man.

The identification of reservoir hosts is also difficult. It is based on the accumulation of evidence to show that the mammal is sufficiently abundant and long-lived to provide a significant source of food for sandflies, that a considerable proportion of these mammals are infected, that the infection runs a long course, that parasites are present in the skin or blood in sufficient numbers to be taken up by a sandfly, that there is intense host-fly contact and, finally, that the parasites in the reservoir host are indistinguishable from those in man. ${ }^{17}$

The leishmaniases can be crudely separated into two groups according to their reservoir hosts. The zoonotic leishmaniases are those in which the reservoir hosts are wild animals (e.g. foxes or jackals), commensals (rodents) or domestic animals (e.g. dogs). Examples of this group are L.m.mexicana and L. major. The anthroponetic leishmaniases are those in which man is the reservoir, for example L.tropica. However, such distinctions are not absolute. L.tropica infection may derive from rodents or dogs and man may constitute a secondary reservoir for L. major! The principal reservoir hosts are shown in Table 1. 
Factors influencing infection frequency. The transmission of leishmaniasis to man is dependent upon ecological factors and therefore varies considerably with time and place. In order for infection to occur man must be exposed to the bite of an infected sandfly. In the New World where leishmania are essentially zoonotic and maintained by forest rodents, the disease is predominantly an occupational one occurring, for example, during construction projects, forestry and military training. In each of these activities, non-immune workers enter the forest where they may encounter infected sandflies. The chance of any individual becoming infected is proportional to the length of time spent in the forest-for example during the construction of the trans-amazonian highway the rate of positive leishmanin skin tests amongst workers increased progressively with longer exposure times. In addition, mini-epidemics may occur if workers enter a 'hot-spot' i.e. a well delimited microfocus where transmission is high as a result of favourable environmental conditions associated with high densities of both reservoirs and sandflies.

In all affected parts of the world, agricultural development projects can lead to large numbers of cases because non-immune workers arrive at the same time as man-made changes to the environment upset the pre-existing zoonotic cycle. Transmission is further increased if during the hot season workers sleep outside on terraces without using nets.

Cutaneous leishmaniasis is predominantly a disease of the poor who have primitive housing and sanitation. The organic debris associated with piles of bricks and stones constitute potential breeding and resting sites for sandflies. These people frequently live at the edge of towns close to the natural sylvatic cycle. A high number of individuals sleeping in one room further attracts peridomestic anthropophilic sandflies. ${ }^{17}$

Periodic fluctuations in the number of cases is well recorded. Although sandflies are present throughout the year in the tropics, each species tends to have an annual cycle and transmission is greatest towards the end of the cycle when the maximum number of parous sandflies are present. There are also great year to year variations, a peak every eight to ten years was noted in a prospective study from Três Bracos, Brazil. ${ }^{26}$

Cutaneous leishmaniasis is also a major problem for the military who, by the nature of their task, bring in large numbers of non-immune individuals to endemic areas. The British garrison in Belize has reported 306 cases between 1978 and 1990 and the Walter Reed army medical centre in Washington treated 70 cases from Panama between 1977 and 1984. ${ }^{27}$ In both cases the soldiers had been undertaking jungle training. During the war between the Islamic Republic of Iran and Iraq, thousands of cases occurred amongst soldiers of both sides. ${ }^{17}$

\section{PREVENTION}

Prevention of cutaneous leishmaniasis involves interrupting the cycle of infection (Figure 2) in at least one place. It is helpful, therefore, to have a comprehensive understanding of the ecology of the cycle in that particular region. In the first instance it is vital to know whether it is anthroponetic or zoonotic. Although control of the sandfly population is important in both types initially, as the intensity of transmission decreases it becomes essential to reduce the reservoir of infection. In anthroponetic leishmaniasis this requires active detection of cases, 
followed by treatment, whereas in zoonotic infections, destruction of the animal reservoir is appropriate. In addition to these measures, people can take individual physical precautions against infection and immunisation is a possibility for some.

Vector control. There are two principal approaches to sandfly controlenvironmental and chemical. Sandflies require dark, damp conditions, feed on vegetable matter and are relatively poor fliers. The population can be greatly reduced by improved sanitation, clearing refuse around buildings, repairing cracks and crevices, clearing followed by levelling of the ground by rolling for 250 metres around dwellings, covering and repairing stream banks and drains to eliminate breeding sites. Further measures such as installing fine mesh screens on doors and windows, good ventilation and sleeping on the first floor of the building can further reduce the chance of being bitten.

Insecticides are widely used and can give immediate and almost complete protection indoors even though the sandflies return if spraying is not repeated regularly. Insecticides with a residual action such as DDT, hexachlorocyclohexane $(\mathrm{HCH})$ and malathion are most useful. DDT and $\mathrm{HCH}$ remain effective for up to one year, whereas malathion lasts only one to four months. Spraying should be directed at doorways, windows, stone walls, animal houses and rubbish heaps. If feasible, spraying for 250 metres around the dwelling is also helpful. Although these insecticides do not affect the eggs, they kill the emerging sandflies.

During malarial control programmes, in which insecticides were widely used as in India and the Mediterranean, leishmaniasis was almost eliminated as a welcome additional benefit. Unfortunately once these programmes stopped, the leishmania returned. Insecticide resistance has also been reported; for example, $P$. papatasi is resistant to DDT in north-east India, and such resistance is likely to become more widespread. WHO provides a simple test to determine the sensitivity of flies to insecticides.

In any control programme, it is important to try and quantify the results of any measures taken. Simple techniques such as quantitative sticky-paper traps or counting the number of sandflies in a pre-selected room after spraying with a knock-down insecticide (e.g. pyrethroid) are sufficient. ${ }^{17}$

Alternative approaches to control which may become available in the future include the use of biological methods, such as sandfly predators or parasites, and genetic methods, such as the release into the natural vector population of sandflies with altered genetic characters (e.g. sterile males). ${ }^{28}$

Reservoir host control. In the case of anthroponetic infections, active case detection followed by treatment is imperative. Such a programme has been dramatically effective in Azerbaijan where cutaneous leishmaniasis had been endemic for many years. It was eliminated in just 10 years by active identification followed by treatment of all affected people (this involved identifying them in hospitals, dispensaries and even by house to house checking), coupled with spraying of all microfoci with a residual insecticide. There are three main problems with this type of programme: (1) good results are obtained initially but as the incidence falls, enthusiasm wanes and a resurgence can occur, (2) particular attention is needed to seek out atypical cases (e.g. recidivans in whom the disease lasts a long time and who may be resistant to the usual treatment) and (3) health 
education must be adequate so that the population understands and are keen to cooperate. ${ }^{28}$

Control of the reservoir hosts for zoonotic disease is also difficult. In the Old World, there have been successes in the USSR where the great gerbil (Rhombomys opimus) has been eliminated in some foci by destruction of its distinctive burrow system by sub-soil ploughing followed by planting. Poisoning has also been used but it is less specific and re-invasion occurs rapidly unless prevented by a natural barrier such as a river. Dogs are more difficult to control; shooting strays helps but it is often unacceptable and poisoning meat with strychnine may inadvertently destroy other animals and birds. Perhaps hygienic disposal by burning of remains from slaughter houses and chicken farms, together with the destruction of stray dogs at rubbish dumps, is a reasonable compromise. ${ }^{17}$

Personal protection. This is particularly relevant when non-immune people enter an endemic region for a relatively short period of time. Simple, but often neglected, measures include application of an insect repellant (e.g. diethyltoluamide which gives up to 8 hours protection) to exposed areas of skin, wearing long trousers and long sleeved shirts, particularly at dusk, and using a bed-net. Although sandflies are able to penetrate a mosquito net it can be rendered very effective by impregnating it with permethrin every three weeks. There is a very fine mesh sandfly net but it is very uncomfortable to use and is therefore usually discarded. ${ }^{28}$

Immunisation. Old World cutaneous leishmaniasis is usually a self-limiting disease which confers long-lasting immunity if allowed to run its natural course. This fact has been known to the indiginous populations of endemic areas for centuries and they have used this knowledge to avoid the disfiguring facial scars of natural infections in two ways. Firstly, some cover their infants but exposed their buttocks to the bites of sandflies so that the ensuing lesion occurred on that site; others inoculated material directly from a cutaneous lesion to the arm or thigh of the recipient. Clearly in these days of HIV infection, the latter practice has even less to commend it! When laboratory culture of leishmania became established, live L.major promastigotes surplanted direct inoculation from lesions and provided protection against both L. major and L.tropica. All these procedures are known as leishmanization; in each case lesions were allowed to develop and run their natural course thereby conferring immunity coupled with a scar on a cosmetically acceptable site. ${ }^{29}$ However, in 2 per cent of cases large or persistent lesions develop which require treatment and there is a small risk of developing leishmaniasis recidivans; leishmanization cannot, therefore, be recommended. Although it had been widely practised in the USSR, Israel and Jordan, it has now largely been abandoned. ${ }^{15}$

No vaccine against any form of leishmaniasis has, so far, been shown to be consistently effective. However, Mayrink's group in Brazil have done pioneering work with a killed promastigote vaccine derived from four strains of leishmania isolated from cutaneous lesions. This is now being produced commercially and preliminary results are encouraging. ${ }^{30} \mathrm{~A}$ different approach is being taken by Convit's group in Venezuela who have a particular interest in immunotherapy with BCG vaccination. They are also attempting to produce a killed promasti- 
gote vaccine but with BCG as an adjuvant. ${ }^{31}$ The identification of maxadilin in sandfly saliva and its crucial role in establishing the infection is yet another avenue which could be explored. If it could be inactivated, then few infections would ever become established.

\section{CONCLUSION}

The leishmaniases are protozoal infections the importance and complexity of which are only just becoming appreciated. In order to take appropriate steps in control, a comprehensive understanding of their ecological niche is required.

\section{REFERENCES}

${ }^{1}$ Elgood S. The early history of the Baghdad boil. $J$ R Asiatic Socy 1934, 519-33.

${ }^{2}$ Gardener PJ. Taxonomy of the genus leishmania: a review of nomenclature and classification. Trop Dis Bull 1977; 174: 1069-88.

${ }^{3}$ Gade (1977). Cited in Walton BC. American cutaneous and mucocutaneous leishmaniasis. In: Peters W, Killick-Kendrick R. (Eds). The Leishmaniases in biology and medicine, Vol 2. London: Academic Press 1987, pp. 637-64.

${ }^{4}$ Cunningham DD. On the presence of peculiar parasitic organisms in the tissue culture of a specimen of Dehli boil. Scientific Memoires of Medical Officers of the Army of India 1885; 1: 21-31.

${ }^{5}$ Firth RH. Notes on the appearance of certain sporozoöid bodies in the protoplasm of an 'oriental sore'. $\mathrm{Br}$ Med J 1891; 1, 60-2.

6 Scott HH. A history of tropical medicine. London: Edward Arnold 1939; pp. 548-69.

${ }^{7}$ Leishman WB. On the possibility of the occurrence of Trypanosomiasis in India. Br Med J 1903; 1: 1252-4.

${ }^{8}$ Donovan C. On the possibility of the occurrence of Trypanosomiasis in India. Br Med J 1903; 2: 79.

9 Wright JH. Protozoa in a case of tropical ulcer (Dehli sore). J Med Res 1903; 10: 472-82.

10 Strang et al. (1915). Cited in Gardener 1977.

${ }^{11}$ Herrer A, Christensen HA. Implications of Phlebotomus sandflies as vectors of Bartonellosis and leishmaniasis as early as 1764 . Science $1975 ; 190$ : 154-5.

12 Rogers L. Preliminary note on the development of Trypanosoma in cultures of the Cunningham-Leishman-Donovan bodies of cachexial fever and Kala-azar. Lancet 1904; 2 : $215-6$.

13 Alder S, Theodor O (1925). Cited in Chlulay JD. Leishmaniasis. In: Strickland GT (Ed). Hunters tropical medicine. 7th ed. Philadelphia: Saunders 1991; pp. 638-55.

14 Sergent E et al. (1921). Cited in Pierini LE. South American Leishmaniasis. In: Simons RDG, Marshall J (Eds). Essays on tropical dermatology. Amsterdam: Exerpta Medical Foundation 1969; pp. $175-203$.

15 Shortt HE, Smith ROA, Swaminath CS, Krishnan KV. Transmission of Indian Kala-Azar by the bite of Phlebotomus argentipes. Indian J Med Res 1931; 18: 1373-5.

16 Alder S, Ber M. The transmission of leishmania tropica by the bite of Phlebotomus papatasi. Indian J. Med Res 1941; 29: 803-9.

17 WHO Expert Committee. Control of the Leishmaniases. Technical Report Series 793. Geneva: WHO 1990.

18 Ashford RW, Desjeux P, de Raadt P. Estimation of population at risk of infection and number of cases of leishmaniasis. Parasitology Today 1992; 8: 104-5.

19 Vieira JB, Lacerda MM, Marsden PD. National reporting of leishmaniasis: the Brazilian experience. Parasitology Today 1990; 6: 339-40.

20 Weigle KA, Valderrama L, Arias AL et al. Leishmanin skin test standardization and evaluation of safety, dose, storage, longevity of reaction and sensitization. Am J Trop Med Hyg 1991; 44: 260-71.

${ }^{21}$ Burgess NRH. Anthropods of medical importance. Chilbolton: Noble 1981; pp. 67-72.

${ }^{22}$ Lewis DJ, Ward RD. Transmission and vectors. In: Peters W, Killick-Kendrick R (Eds) The leishmaniases in biology and medicine, Vol 1. London: Academic Press 1987; pp. $235-62$.

23 WHO Expert Committee. The leishmaniases. Technical Report Series 701. Geneva: WHO 1984.

${ }^{24}$ Lerner E. Vector biology in leishmaniasis transmission: new findings. Proceedings 18 th World Congress of Dermatology, New York 12-18 June 1992. 
25 Theodos CM, Ribeiro JMC, Titus TG. Analysis of enhancing effect of sandfly saliva on leishmania infection in mice. Infection and Immunity 1991; 59: 1592-8.

${ }^{26}$ Jones TC, Johnson WD, Barretto AC, Largo E et al. Epidemiology of American cutaneous leishmaniasis due to leishmania braziliensis braziliensis. J Infect Dis 1987; 156: 73-83.

${ }^{27}$ Ballou WR, McClain JB, Gordon DM, Shanks GD, Andujar J, Berman JD, Chulay JD. Safety and efficacy of high dose sodium stibogluconate therapy of American cutaneous leishmaniasis. Lancet 1987; ii: $13-16$.

28 Vioukov VN. Control of transmission. In: Peters W, Killick-Kendrick R (Eds). The leishmaniases in biology and medicine, Vol 2. London: Academic Press 1987; pp. 909-28.

29 Gunders AE. Vaccination: past and future role in control. In: Peters W, Killick-Kendrick R (Eds). The leishmaniases in biology and medicine, Vol 2. London: Academic Press 1987; pp. 929-42.

${ }^{30}$ Modabber F. Development of vaccines against leishmaniasis. Scand J Infect Dis, Suppl. 1990; 76: 72-8.

${ }^{31}$ Playfair JHL, Blackwell JM, Miller HRP. Modern vaccines-parasitic diseases. Lancet 1990; 335: 1263-6. 


\title{
Hepatotoxicity of sodium stibogluconate therapy for American cutaneous leishmaniasis
}

\author{
N. C. Hepburn ${ }^{1}$, I. Siddique ${ }^{2}$, A. F. Howie $^{3}$, G. J. Beckett ${ }^{3}$, and P. C. Hayes ${ }^{2}$ Departments of ${ }^{1}$ Dermatology,
}

${ }^{2}$ Medicine and ${ }^{3}$ Clinical Biochemistry, The Royal Infirmary, Edinburgh, EH3 9YW

\begin{abstract}
Sodium stibogluconate is the mainstay of treatment for all forms of leishmaniasis. Therapy is associated with an increase in serum aminotransferases. In this study liver damage was assessed during treatment of American cutaneous leishmaniasis with sodium stibogluconate and also in a control group given aminosidine. In addition to standard liver function tests, acute hepatocellular damage was assessed by measuring plasma glutathione S-transferase $\mathrm{B}_{1}$ (GST), and hepatic metabolic capacity was assessed by a caffeine clearance (CCL) test, before, during and after treatment. Thirteen patients were treated; 5 received sodium stibogluconate, 6 received aminosidine and a further 2 patients received aminosidine followed by sodium stibogluconate. Treatment with sodium stibogluconate was associated with an increase in both alanine aminotransferase (ALT) and GST and a fall in the CCL, indicating both hepatocellular damage and functional impairment. Six weeks after treatment had stopped ALT and GST had returned to pre-treatment levels and the CCL remained depressed in only one patient. Patients given aminosidine did not show any evidence of liver damage. Sodium stibogluconate is associated with significant hepatocellular damage and hepatic functional impairment. However, this is rapidly reversible on drug withdrawal. We suggest that liver function is monitored throughout treatment and that patients with pre-existing liver disease receive alternative treatment.
\end{abstract}

\section{Introduction}

Sodium stibogluconate, a pentavalent antimonial, remains the mainstay of treatment for both visceral and cutaneous leishmaniasis (WHO, 1990). In recent years higher doses have been administered and courses have lasted longer in order to achieve greater efficacy (BAL LOU et al., 1987; WHO, 1990; HEPBURN et al., 1993). Transient abnormalities of serum aminotransferases have been recorded during treatment for cutaneous leishmaniasis (CL) and these appear to be dose-related (HEPBURN et al., 1993); however, it is not known whether sig. nificant and/or permanent liver damage results from sodium stibogluconate therapy.

Increased serum aminotransferase levels reflect hepatocellular damage but there is little correlation between their absolute level and the degree of damage (HAYES \& BOUCHIER, 1989). Glutathione S-transferase $B_{1}$ (EC 2.5.1.18; GST) is distributed throughout the liver lobule whereas aminotransferases are confined to the periportal cells. Plasma GST levels increase when any zone of the liver is damaged whilst ALT and aspartate aminotransferase (AST) are often normal after centrilobular damage. GST is a more sensitive marker of acute liver damage in disease and following paracetamol overdose (BECKETT et al., 1985; BECKETT \& HAYES, 1993). Although hepatocellular injury indicates liver damage, it is more important to determine whether or not hepatic metabolic capacity is impaired. The liver's large metabolic capacity, particularly in fit young men, means that standard indicators of hepatic synthetic capacity, such as the prothrombin time or serum albumen, do not become abnormal until damage is extensive (PRESIG, 1986). The clearance rate of caffeine from the blood following oral administration directly reflects the amount of functioning liver tissue. Caffeine is almost exclusively metabolized in the liver and there is no overlap in clearance rates between healthy subjects and patients with cirrhosis (RENNER et al., 1984; PrEsig, 1986; HAYES \& BOUCHIER, 1989; KALOW \& TANG, 1993). We report the results in a prospective controlled study in which hepatocellular damage and liver function were assessed in patients treated with sodium stibogluconate for American CL.

\section{Methods}

An open prospective randomized study comparing the efficacy and toxicity of sodium stibogluconate with ami-

Address for correspondence: Dr N. C. Hepburn, Department of Dermatology, Level 4-Lauriston Building, The Royal Infirmary, Edinburgh, EH3 9YW nosidine for treatment of CL is in progress at the army hospital in Edinburgh, UK. All the patients are British soldiers who have contracted CL in Belize. This study had ethical approval from the Army Medical Services Research Executive and each soldier gave written informed consent. In each case a full medical history was obtained and a physical examination performed and the diagnosis was confirmed by demonstration of the parasite by histology and/or culture. Patients received $20 \mathrm{~d}$ treatment with either sodium stibogluconate (Pentostam ${ }^{8}$, Wellcome, UK), $20 \mathrm{mg} / \mathrm{kg} / \mathrm{d}$, or aminosidine (Gabbro-

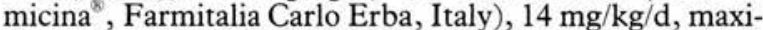
mum $1 \mathrm{~g} / \mathrm{d}$. Both drugs were given by intravenous infusion; sodium stibogluconate was given undiluted at 1 $\mathrm{mL} / \mathrm{min}$ whereas aminosidine was diluted in $400 \mathrm{~mL}$ of normal saline and given over $4 \mathrm{~h}$. Laboratory monitoring consisting of a full blood count, urea and electrolytes levels, liver function tests (LFTs), prothrombin time, cardiac enzymes and electrocardiography was performed before treatment, weekly during treatment, and at intervals for 6 weeks after treatment. Ulcers were considered to have healed when they had re-epithelialized and the scar was flat and non-indurated.

In 13 consecutive patients, in addition to the routine LFTs which were measured using an automatic analyser (BM/Hitachi $747^{\circ}$, Boehringer Mannheim, Mannheim, Germany), plasma GST was measured by radioimmunoassay (HAYES et al., 1983) and a caffeine clearance

Table. Clinical features and response to treatment of patients with American cutaneous leishmaniasis treated with either sodium stibogluconate or aminosidine

\begin{tabular}{|c|c|c|}
\hline & \multicolumn{2}{|c|}{ Treatment } \\
\hline & $\begin{array}{c}\text { Sodium } \\
\text { stibogluconate }\end{array}$ & Aminosidine \\
\hline No. of patients & 7 & 8 \\
\hline Mean age (years) & $23 \cdot 6(\mathrm{SD} 3 \cdot 3)$ & $25 \cdot 0(\mathrm{SD} 2 \cdot 7)$ \\
\hline Mean weight $(\mathrm{kg})$ & $72 \cdot 0(\mathrm{SD} 5 \cdot 9)$ & $72 \cdot 2(\mathrm{SD} 6 \cdot 5)$ \\
\hline \multicolumn{3}{|l|}{ Lesion } \\
\hline Mean no. & $1.3(\mathrm{SD} 0.8)$ & $1.3(\mathrm{SD} 0.7)$ \\
\hline Mean duration (weeks) & $16.6(\mathrm{SD} 8.5)$ & $10.6(\mathrm{SD} 4.6)$ \\
\hline Mean $\operatorname{size}^{\mathrm{a}}(\mathrm{mm})$ & $16 \cdot 0(\mathrm{SD} 2 \cdot 7)$ & $10 \cdot 6(\mathrm{SD} 4 \cdot 6)$ \\
\hline \multicolumn{3}{|l|}{ Site } \\
\hline Limbs & 5 & 6 \\
\hline Head/neck & 3 & 3 \\
\hline Trunk & 0 & 1 \\
\hline No. with amastigotes & 7 & 8 \\
\hline No. with positive culture & 3 & 8 \\
\hline No. clinically healed & 6 & 5 \\
\hline
\end{tabular}

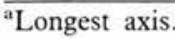




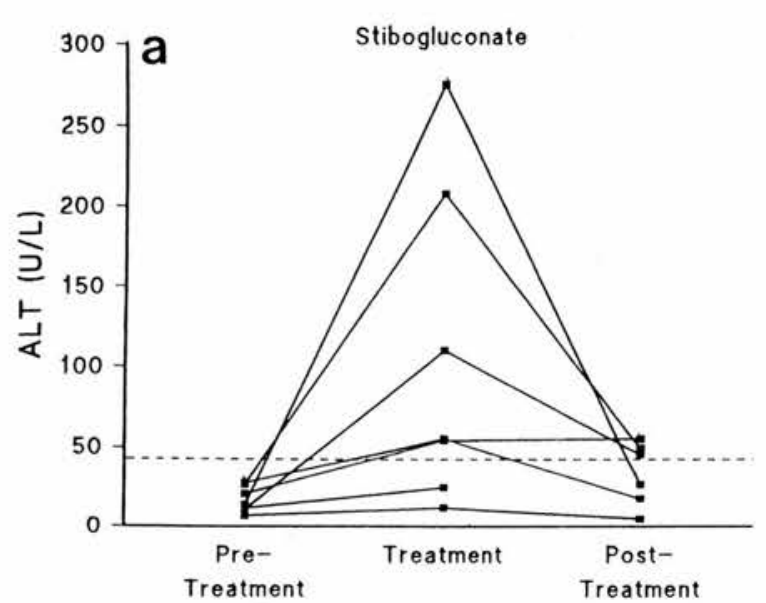

Stibogluconate

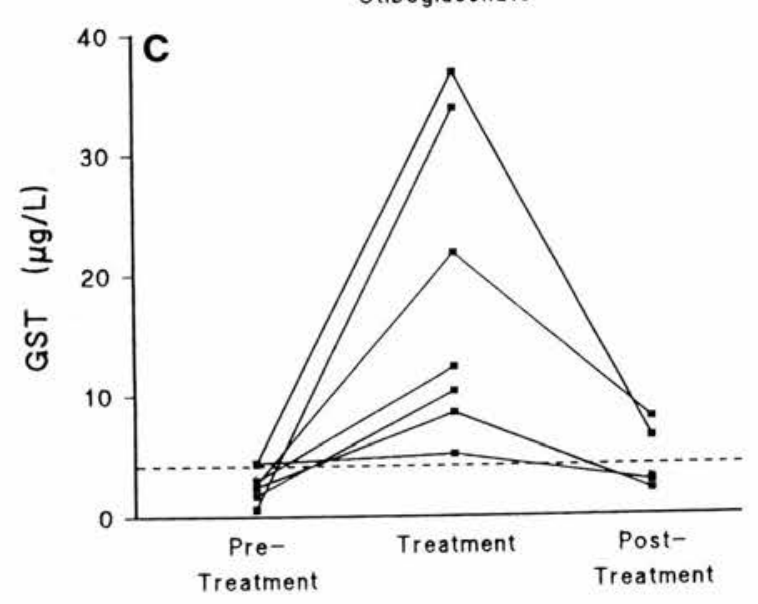

Stibogluconate

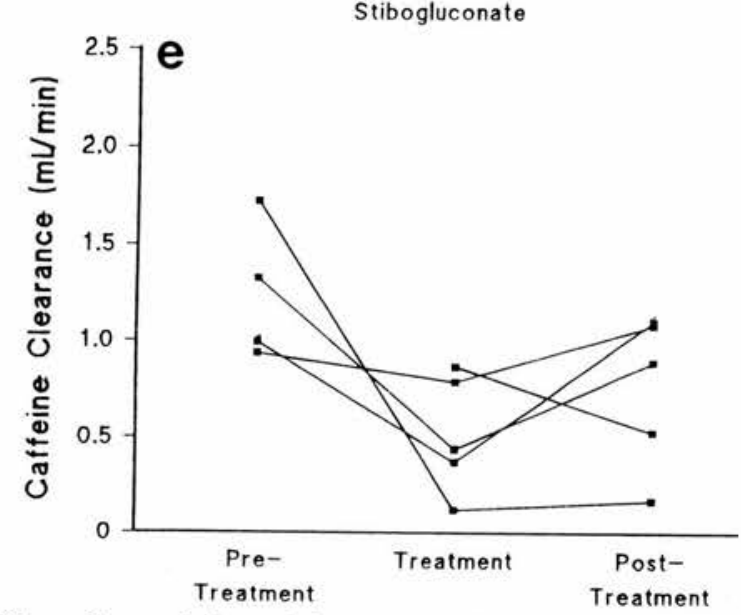

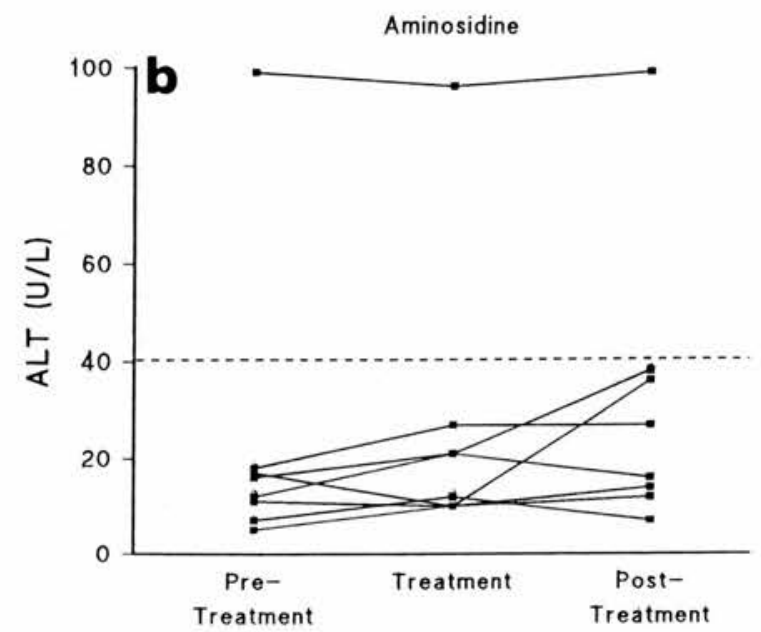
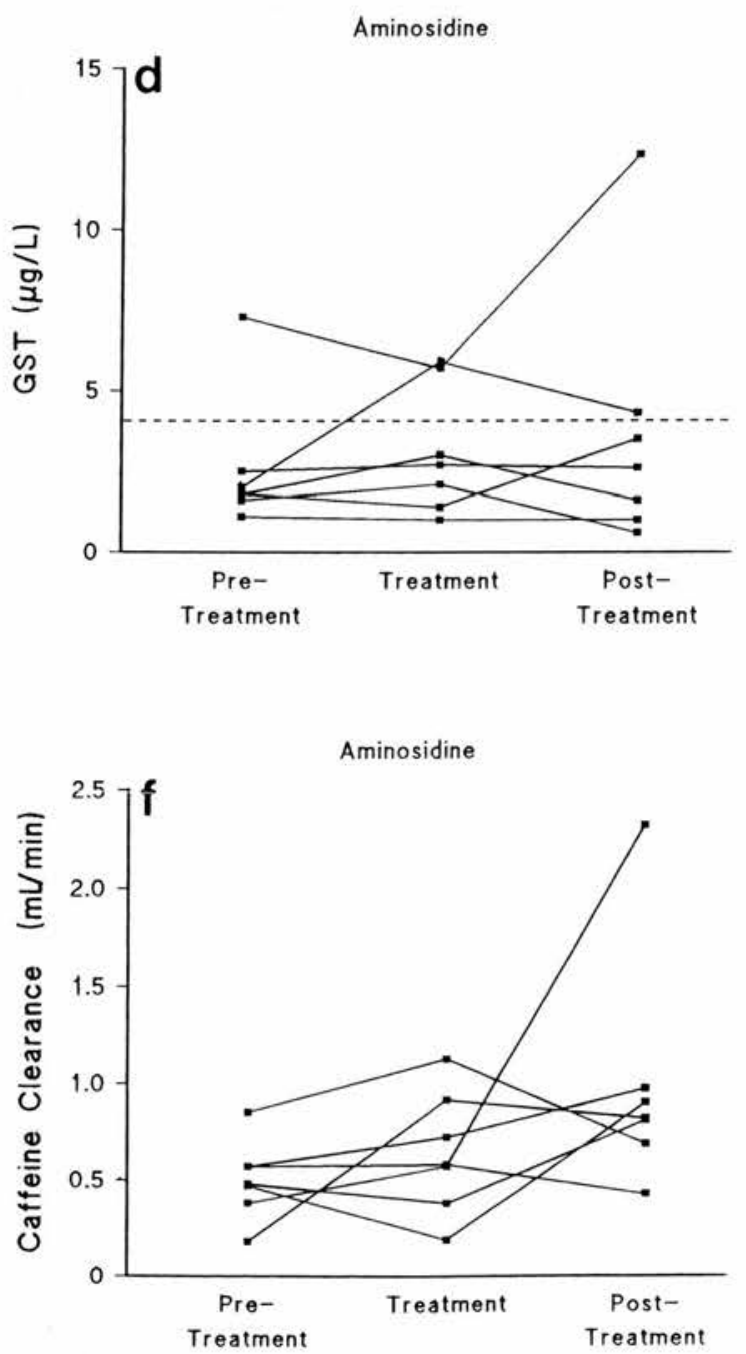

Figure. Changes during and after treatment with sodium stibogluconate (a, c,e) or aminosidine (b, d, f) of plasma alanine aminotransferase (ALT; a, b) and plasma glutathione $S$-transferase $B_{1}(G S T ; c, d)$ levels, and caffeine clearance $(e, f)$, in patients with American cutaneous leishmaniasis.

(CCL) test was performed before treatment, at the end of treatment, and 6 weeks after treatment had stopped. The CCL was performed as described by SETCHELl et al. (1987). The patients were fasted overnight and allowed only water to drink. A single dose of $225 \mathrm{mg}$ of caffeine was administered in a cup of decaffeinated coffee which was consumed over $5 \mathrm{~min}$. Blood samples were taken at $0,60,90,120$ and $180 \mathrm{~min}$ and the caffeine level was measured by high-performance liquid chromatography using an Altex $320^{\circ}$ (Anachem, Luton, UK). Five of these patients had been treated with sodium stibogluconate, 6 with aminosidine, and 2 patients had been treated 
first with aminosidine and then, because of their poor response, with sodium stibogluconate after a break of 6 weeks. The clinical features of these patients at the start of treatment, and their response to treatment, are shown in the Table. Changes in ALT, GST and CCL before and at the end of treatment and also before and 6 weeks after treatment were analysed by a Wilcoxon matched-pairs test.

\section{Results}

The activity of ALT, the concentration of GST, and the CCLs for each treatment group are shown in the Figure. Sodium stibogluconate therapy was associated with a significant rise in ALT $(P<0.05)$ and GST $(P<0.05)$ and a non-significant fall in CCL in every patient. Log ALT correlated with $\log$ GST $(r=0 \cdot 87, n=40)$. The prothrombin time and serum albumin level did not change. Six weeks after treatment stopped, ALT and GST levels had returned to near pre-treatment values in every patient, as had the CCL in all patients except one; although this patient had the greatest fall in CCL, it then increased, and his GST had increased only from $1.7 \mu \mathrm{g} / 1$ to $10 \cdot 3 \mu \mathrm{g} / 1$

Aminosidine therapy was not associated with a rise in ALT or a fall in CCL. In one patient a small rise in GST was noted and in a further patient, who was reported to consume large quantities of alcohol, the ALT and GST were elevated before treatment, fell during treatment (alcohol was forbidden during treatment, which was supervized in hospital), but rose again after discharge. Acute alcohol ingestion in heavy drinkers leads to increased GST levels and their basal GST levels are often raised (BECKETT \& HAYES, 1993).

\section{Discussion}

Although increases in serum aminotransferases, indicative of hepatocellular damage, have been reported during sodium stibogluconate treatment (BALLOU et al., 1987; HEPBURN et al., 1993), the clinical significance of this is not known. It is not possible to separate the toxic from the therapeutic effects on the liver in visceral leishmaniasis, but this should not be a problem in cutaneous disease. Nevertheless, a further control group of patients with CL who were treated with aminosidine, which is not hepatotoxic, was incorporated into this study. GST is a very sensitive and specific indicator of hepatocellular damage, and its short half-life in plasma allows early recognition of the cessation of active cellular damage (BECKETT \& HAYES, 1993). Treatment with sodium stibogluconate, but not with aminosidine, resulted in significant increases in ALT and GST, confirming that sodium stibogluconate causes hepatocellular damage. The elevation of both ALT and GST indicated that damage was occurring either in all zones of the liver or that it was confined to the periportal hepatocytes. The fall in both ALT and GST to pre-treatment levels 6 weeks after treatment stopped indicated that the damage had also stopped.

The fall in CCL during treatment with sodium stibogluconate indicated that hepatic metabolic capacity was impaired. Although the numbers in this study were insufficient to achieve statistical significance, CCL fell in every case. However, this change appeared to be reversible once treatment had been stopped. Although the fall in CCL could have been accounted for by selective inhibition of cytochrome P-450 1A2, the principle enzyme by which caffeine is metabolized (KALOW \& TANG, 1993), the concomitant changes in ALT and GST indicated that hepatocellular injury was the probable mech- anism. These changes were not observed in patients treated with aminosidine, therefore sodium stibogluconate therapy is clearly implicated as the cause.

Hepatotoxicity must therefore be added to the list of complications associated with sodium stibogluconate therapy. In most cases of CL this is of limited clinical significance, but for patients with pre-existing liver disease or visceral leishmaniasis alternative therapy should be considered, and in all cases the concurrent use of other potentially hepatotoxic agents avoided. The ALT level gives a practical indication of the severity of acute hepatocellular damage but will not detect any patient who sustains permanent damage. A less toxic treatment is needed. Systemic aminosidine has been shown to be as efficacious as sodium stibogluconate in treating visceral leishmaniasis in one study (CHUNGE et al., 1990) and it has been used successfully as an ointment in 'Old World' CL (EL-ON et al., 1992). It appears to be free of hepatoxic effects, but its efficacy in 'New World' CL has yet to be determined.

\section{References}

Ballou, W. R., McClain, J. B., Gordon, D. M., Shanks, G. D., Andujar, J. Berman, J. D. \& Chulay, J. D. (1987). Safety and efficacy of high-dose sodium stibogluconate therapy of American cutaneous leishmaniasis. Lancet, ii, 13-16.

Beckett, G. J. \& Hayes, J. D. (1993). Glutathione S-transferases: biomedical applications. Advances in Clinical Chemistry, $30,279-378$.

Beckett, G. J., Chapman, B. J., Dyson, E. H. \& Hayes, J. D. (1985). Plasma glutathione S-transferase measurement after paracetamol overdose: evidence for early hepatocellular damage. Gut, 26, 26-31.

Chunge, C. N., Owate, J., Pamba, H. O. \& Donno, L. (1990). Treatment of visceral leishmaniasis in Kenya by aminosidine alone or combined with sodium stibogluconate. Transactions of the Royal Society of Tropical Medicine and Hygiene, 84, 221225 .

El-On, J., Halevy, S., Grunwald, M. H. \& Weinrauch, L. (1992). Topical treatment of old world cutaneous leishmaniasis caused by Leishmania major: a double blind control study. Fournal of the American Academy of Dermatology, 27, 227-231.

Hayes, P. C. \& Bouchier, I. A. D. (1989). Liver function tests in clinical practice: their uses and limitations. Clinical Chemistry and Enzyme Communications, 2, 23-34.

Hayes, J. D., Gilligan, D. Chapman, B. J. \& Beckett, G. J. (1983). Purification of human hepatic glutathione S-transferase and the development of a radioimmunoassay for its measurement in plasma. Clinica Chimica Acta, 134, 107-121.

Hepburn, N. C., Tidman, M. J. \& Hunter, J. A. A. (1993). Cutaneous leishmaniasis in British troops from Belize. British 7ournal of Dermatology, 128, 63-68.

Kalow, W. \& Tang, B. K. (1993). The use of caffeine for enzyme assays: a critical appraisal. Clinical Pharmacology and Therapeutics, 53, 503-514.

Presig, R. (1986). Clinical evaluation of liver function. In: Recent Advances in Hepatology, vol. 2, Thomas, H. C. \& Jones, E. (editors). Edinburgh: Churchill Livingstone, pp. 1-12.

Renner, E., Weitholz, H., Huguenin, P., Arnaud, M. \& Presig, R. (1984). Caffeine: a model compound for measuring liver function. Hepatology, 4, 38-46.

Setchell, K. D. R., Welsh, M. B., Klooster, M. J. \& Ballistreri, W. F. (1987). Rapid high-performance liquid chromatography assay for salivary and serum caffeine following an oral load: an indicator of liver function. Fournal of Chromatography, 385, 267-274

WHO (1990). Control of the Leishmaniases. Geneva: World Health Organization, Technical Report Series, no. 793

Received 6 fuly 1993; revised 6 September 1993; accepted for publication 8 September 1993 


\title{
The effect of ivermectin treatment on the antibody response to antigens of Onchocerca volvulus
}

A. J. Gillespie ${ }^{1}$, S. Lustigman ${ }^{2}$, A. R. Rivas-Alcala ${ }^{3}$ and J. E. Bradley ${ }^{1}{ }^{1}$ Department of Biology, Imperial College of Science, Technology and Medicine, Prince Consort Road, London, SW7 2BB, UK; ${ }^{2}$ Laboratory of Virology and Parasitology, The Lindsey F. Kimball Research Institute of the New York Blood Center, New York, NY, USA; ${ }^{3}$ Deceased; formerly of CIES, Carretera Panamericana y Periferico Sur S/N, San Christobal de Las Casas, Chiapas, Mexico

\begin{abstract}
The effect of the microfilaricidal drug ivermectin on the antibody response to a detergent extract of adult Onchocerca volvulus (OvAg) and a number of specific recombinant peptides was examined. Three of the peptides were combined in a serodiagnostic 'cocktail' and the effect of ivermectin on the diagnostic performance of this assay was assessed. Immunoglobulin $(\mathrm{Ig}) \mathrm{G}_{1}$ serum levels in response to OvAg significantly decreased following ivermectin treatment. The antibody response to only one recombinant peptide (OvMBP29) was significantly affected, with IgG levels decreasing following treatment. Levels of total IgE increased following treatment. No correlation was observed between initial antibody level (or change in antibody level) and any adverse reaction to treatment. The serodiagnostic 'cocktail' was $100 \%$ sensitive before and after the use of ivermectin. A serodiagnostic assay using specific recombinant peptides can be used to evaluate infection in the absence of dermal microfilariae in areas where ivermectin is used.
\end{abstract}

\section{Introduction}

The use of ivermectin has revolutionized the treatment of onchocerciasis. Originally developed as an anti-parasitic drug for use with livestock, the microfilaricidal activity of ivermectin against Onchocerca volvulus infection in humans was first reported by AzIZ et al. (1982a, 1982 b). Following extensive human trials, the French Government first approved the use of ivermectin in 1987 and it is now the drug of choice for onchocerciasis treatment world-wide.

Diagnosis of $O$. volvulus currently involves examination of skin snips for microfilariae ( $\mathrm{mf}$ ), a technique rendered ineffective in areas where microfilaricidal ivermectin is used; furthermore, skin snip diagnosis does not detect prepatent infections and is insensitive in low level patent infections (BUCK, 1974). As an alternative approach, a serodiagnostic test was developed employing recombinant peptides specifically recognized by sera from persons infected with $O$. volvulus (see BRADLEY et al., 1991, 1993a). Two of these peptides are being considered by the World Health Organization (WHO) for use in a diagnostic assay which could replace, or provide an additional tool to, the use of skin snip diagnosis within the Onchocerciasis Control Programme (OCP) (RAMACHANDRAN, 1993). As the OCP has recently introduced ivermectin treatment as a complementary control measure to vectoricidal spraying, it is important to assess any change in the performance of the diagnostic assay that may result from the use of the drug.

Adverse reactions to ivermectin treatment range from pruritis to more serious conditions such as hypotension, although severe reactions such as the latter are rare (DE SOLE et al., 1989; WhITWORTH et al., 1992). As is true for most of the pathology associated with $O$. volvulus infection, the adverse reactions to treatment with the previous drug of choice, diethylcarbamazine, and probably those to ivermectin treatment, are mediated by inappropriate immune responses of the host (OTTESEN, 1985). It is therefore important to assess any influence ivermectin may have on host immune responses. STEEL et al. (1991) reported changes in antibody responses to an extract of adult $O$. volvulus following ivermectin treatment. A more complete picture may be provided by the examination of antibody responses to stage- and species-specific recombinant peptides of $O$. volvulus.

The primary aim of the present study was to examine the effect of ivermectin treatment on the diagnostic performance of a 'cocktail' of recombinant peptides, previously shown to exhibit high levels of sensitivity and specificity (BRADLEY et al., 1993a). The study design

Address for correspondence: Dr Janette E. Bradley, Department of Biology, Imperial College of Science, Technology and Medicine, Prince Consort Road, London, SW7 2BB, UK. also enabled the investigation of any association between changes in antibody levels and the development of adverse reaction to treatment.

\section{Materials and Methods \\ Study population}

Eighty randomly selected males from the $O$. volvulusendemic region of southern Chiapas in Mexico participated in a single 'blind' placebo-controlled trial of ivermectin. All patients were aged between 12 and 60 years and had the presence of dermal mf confirmed by skin snip examination. Doses of ivermectin (150-220ug) or placebo were given 3 times at intervals of 6 months. Patients were clinically examined each time and serum samples were collected before, and 6 months after, the initial treatment and 6 months after the second treatment.

\section{Control sera}

Non-endemic control sera were obtained from 48 individuals living in an area of Venezuela known to be free from $O$. volvulus and Wuchereria bancrofti transmission.

\section{Antigens}

$\mathrm{O}$. volvulus detergent extract. Aduit female $O$. volvulus were dissected from nodules obtained from patients resident in the study area. A detergent extract (OvAg) was prepared as described previously (TRENHOLME et al., 1994).

Recombinant proteins. Three recombinant antigens, OvMBP $/ 10$, OvMBP $/ 11$ and OvMBP/29, had been previously selected for their specificity and sensitivity as diagnostic probes (BRADLEY et al., 1991, 1993a). They were over-expressed and purified as described by BRADLEY et al., (1993a), and are referred to as the diagnostic 'cocktail' when used in combination.

A fourth recombinant peptide, Ov103, not included in the diagnostic assay, was over-expressed and purified in the pGEX-1N plasmid vector, producing a fusion polypeptide with the carboxy terminus of the Schistosoma japonicum glutathione S-transferase protein (GST) (SMITH et al., 1987; LUSTIGMAN et al., 1992b).

OvMBP/10 (corresponding to Ov7; Lustigman et al., 1991 ) is found in the L3, L4 adult stages of the worm and Ov103 is found in the adult female and $\mathrm{mf}$ stages (LUSTIGMAN et al., 1992b). Localization studies for OvMBP/11 and OvMBP/29 are incomplete, although both are known to be present in adult female worms (BRADLEY et al., 1991).

\section{Statistical evaluation}

The frequency distributions of isotype responses to some recombinant antigens did not permit the use of par- 


\title{
Cardiac effects of sodium stibogluconate: myocardial, electrophysiological and biochemical studies
}

\author{
N.C. HEPBURN ${ }^{1}$, J. NOLAN ${ }^{2}$, L. FENN ${ }^{2}$, R.M. HERD ${ }^{1}$, J.M.M. NEILSON ${ }^{3}$, \\ G.R. SUTHERLAND ${ }^{2}$ and K.A.A. FOX ${ }^{2}$
}

From the Departments of ${ }^{1}$ Dermatology, ${ }^{2}$ Cardiology, and ${ }^{3}$ Medical Physics, The Royal Infirmary, Edinburgh, UK

Received 29 March 1994; Accepted 16 May 1994

\section{Summary}

The pentavalent antimonial sodium stibogluconate is the mainstay of anti-leishmanial therapy. Sodium stibogluconate is less cardiotoxic than antimony and the trivalent derivatives, but has been associated with dose-related electrocardiographic changes. The effect of the currently-used regimen of sodium stibogluconate $(20 \mathrm{mg} / \mathrm{kg} / \mathrm{day}$ for 20 days) on cardiac function is uncertain. We studied 12 soldiers, mean age $\mathbf{2 4}$ years, with proven cutaneous leishmaniasis treated with this regimen. There were no significant changes in echocardiographic indices of left ventricular systolic or diastolic function during treatment. Indices of myocardial electrical stability (heart-rate variability and episodes of overt supraventricular and ventricular arrhythmias) were unchanged, but there was a reversible decrease in $\mathrm{T}$-wave amplitude during treatment. Systolic and diastolic blood pressure fell and the heart rate increased during treatment.

This regimen of sodium stibogluconate does not measurably impair left ventricular systolic or diastolic function. Minor T-wave changes occur during treatment, but there is no increase in arrhythmia frequency or change in heart-rate variability. In most young fit patients, this regimen has no cardiac side-effects. However, idiosyncratic reactions cannot be excluded, and patients with malnutrition, impaired renal function or pre-existing heart disease may be more sensitive to any cardiotoxic properties of sodium stibogluconate.

\section{Introduction}

Leishmaniasis is a major world health problem affecting some $12 \mathrm{~m}$ people. The pentavalent antimonials, sodium stibogluconate and meglumine antimoniate, are the mainstay of treatment. ${ }^{1}$ Antimony is cardiotoxic. In dogs it reduces myocardial contractile force ${ }^{2}$ and in man the trivalent antimonials have been associated with hypotension, bradycardia, ventricular tachycardia, syncope, electrocardiographic (ECG) changes (deformed P waves, QT prolongation and T-wave flattening) and occasionally sudden death. ${ }^{3-8}$ Sodium stibogluconate was developed as a less cardiotoxic alternative ${ }^{8,9}$ but ECG abnormalities (T-wave flattening and QTc prolongation) have been reported $^{10-13}$ and appear to be dose-related $;^{12}$ similar changes have been observed during meglumine antimoniate therapy. ${ }^{14}$ It is not known if these occur in association with dysrhythmias, other markers of myocardial electrical instability, or changes in cardiac function. Since current regimes recommend the administration of prolonged high-dose sodium stibogluconate to increase efficacy, ${ }^{1,15-17}$ it is important to determine whether these are associated with significant deleterious effects on cardiac function. The aim of this study was to examine cardiac function in detail during treatment of cutaneous leishmaniasis (CL) with the current prolonged high-dose course of sodium stibogluconate $(20 \mathrm{mg} / \mathrm{kg} / \mathrm{day}$, without an upper daily dose limit, for 20 days) using haemo-

Address correspondence to Major N.C. Hepburn RAMC, Department of Dermatology, Cambridge Military Hospital, Aldershot GU11 2AN 
dynamic, biochemical, electrocardiographic and echocardiographic techniques.

\section{Methods}

\section{Patients and drug regimen}

Twelve British soldiers, aged between 20 and 30 years, who had contracted $\mathrm{CL}$ during jungle training in Belize, were studied. They formed a subgroup of patients participating in an open, prospective trial of sodium stibogluconate vs. aminosidine fully described elsewhere. ${ }^{18}$ They all gave informed consent, and their characteristics are shown in Table 1. None had a history of cardiac or renal disease, diabetes mellitus or alcohol abuse. Those receiving concurrent medication were excluded from the study. All patients had a history of exposure to the parasite, a typical skin lesion, and histological evidence of infection (Leishman-Donovan bodies seen in all 12 patients, positive culture in 9 patients). Treatment with sodium stibogluconate $20 \mathrm{mg} / \mathrm{kg} /$ day without an upper daily dose limit (Pentostam, Wellcome, batch no. A1058A, manufactured January 1991 and stored at below $25^{\circ} \mathrm{C}$ ) was administered by intravenous infusion at $1 \mathrm{ml}(10 \mathrm{mg}) / \mathrm{min}$ for 20 consecutive days.

\section{Blood pressure and biochemical measurements}

These were made before treatment started (day 0), weekly during treatment (days 7,14 ), on the last day of treatment (day 20), 2 weeks after treatment stopped (day 34 ) and 6 weeks after treatment stopped (day 62). Blood pressure was measured at rest using a mercury sphygmomanometer (diastolic phase V). Creatine kinase and urea-stable lactate dehydrogenase were measured using commercial photo-

Table 1 Characteristics of the patients

\begin{tabular}{lc}
\hline Number of patients & 12 \\
Mean age (years) & 23.7 (SD 3.1) \\
Mean weight $(\mathrm{kg})$ & 71.7 (SD 3.1) \\
Mean number of lesions & 1.25 (SD 0.6) \\
Mean duration of lesions (weeks) & 21.3 (SD 9.9) \\
Mean size* (mm) & 16.7 (SD 5.0) \\
Site of lesions: & \\
$\quad$ limbs & 11 \\
$\quad$ head \& neck & 3 \\
$\quad$ trunk & 1 \\
LD bodies seen & $12 / 12$ \\
Positive culture & $9 / 12(6 \mathrm{Lbb}, 3 \mathrm{Lmm})$ \\
Lesion healed after treatment & $11 / 12$ \\
\hline
\end{tabular}

* Longest axis. metric assays (Monarch, Allied Instrumentation Laboratory).

\section{Electrophysiological measurements}

Standard resting ECGs were obtained using a Minigraph 12-lead recorder (Cardiac Recorders) before treatment, weekly during treatment and at 2 and 6 weeks after treatment. The electrocardiograms were inspected and the cardiac axis determined using standard criteria. ${ }^{19}$ The electrocardiographs were then projected on to a screen to produce a magnification of $\times 5$. Using the isoelectric PR and post-T-wave segments as the baseline, the height of the $R$ wave in $V 1$ and the sum of the $T$ waves in V4-V6 were measured directly using a ruler and used as quantitative measurements of changes in QRS and T-wave magnitude.

Twenty-four-hour ambulatory electrocardiograms were obtained from each patient during sedentary activity before treatment, on the last day of treatment (day 20) and 6 weeks after treatment stopped (day 62) using a miniature tape recorder (Tracker, Reynolds Medical). Times of going to bed and getting up were noted by the patients. The tapes were analysed using a Pathfinder arrhythmia analyser (Reynolds Medical) at 120 times the original recording speed. Supraventricular and ventricular ectopic beats were detected due to differences in their timing and morphology, compared with continuously updated characteristics of normal sinus beats. ${ }^{20}$ Each analysis was monitored throughout by a specialist technician to confirm the ectopic beats and scrutinize P-wave morphology to exclude any episodes of nonsinus rhythm. Where $<24 \mathrm{~h}$ of recording was suitable for analysis, the totals were normalized to the equivalent of $24 \mathrm{~h}$. Recordings shorter than $18 \mathrm{~h}$, or with $<40 \%$ of the tape suitable for analysis were rejected. The mean waking and sleeping heart rates over the $24 \mathrm{~h}$ period were calculated, and the number of ectopic beats per $24 \mathrm{~h}$ was counted.

Changes in autonomic activity occur in association with changes in left ventricular function ${ }^{21}$ and may be important in the pathogenesis of ventricular tachyarrhythmias. ${ }^{22}$ During arrhythmia analysis, each normal RR interval was measured, the mean RR interval was calculated, and the 24-h standard deviation (SD) of RR intervals was measured as a general index of sympathovagal activity. ${ }^{23,24} \mathrm{~A}$ specific measure of parasympathetic activity was obtained by the $\mathrm{NN}_{50}$ time domain index, in which each time a normal RR interval exceeded $50 \mathrm{~ms}$, a count was registered and the results were presented as a total 24-h RR count $\left(\mathrm{NN}_{50}\right.$ count). ${ }^{25}$

\section{Assessment of left ventricular function}

This was done by echocardiography in all patients before treatment, on the last day of treatment (day 
20) and 6 weeks after treatment (day 62). All echocardiographic measurements were obtained by the same operator (LF), using identical equipment on each occasion, in a temperature-controlled laboratory at $22{ }^{\circ} \mathrm{C}$. Following an overnight fast, the patients rested in the standard left lateral position until their heart rate stabilized. Heart rate was recorded from a continuous ECG (lead 2) and respiration with a respiratory probe. High-quality simultaneous recordings of the echocardiogram, phonocardiogram, electrocardiogram and respiration tracing were obtained using an Accuson 128/XP10 scanner (Allison). Phonocardiograms were obtained with a highimpedance piezo-electric microphone with a lowfrequency filter, positioned at the base of the heart. Aortic valve closure was taken as the start of the first high-frequency vibration of the aortic component of the second heart sound, checked for validity with the aortic echocardiogram and the aortic artefact on the Doppler recording. Two-dimensional, M-mode and Doppler echocardiograms were obtained with a hand-held $3.5 \mathrm{MHz}$ phased array transducer during quiet respiration. Simultaneous recordings were printed onto thermal paper at $100 \mathrm{~mm} / \mathrm{s}$. Clear continuous echoes from the septum and posterior wall, and easily visible recordings of aortic valve opening and closure were obtained in all patients. Pulsed Doppler recordings of the mitral inflow velocity profile were obtained by positioning the sample volume at the level of the mitral leaflet tips in diastole in the apical four-chamber view. Care was taken to obtain a smooth velocity curve with welldefined peaks and minimal spectral broadening. Recordings were analysed off-line using a sonic pen and a digitizing tablet (Sunnagraphics $0.1 \mathrm{~mm}$ resolution grid) interfaced to a microcomputer (Sun 3/180 workstation) using the measurement techniques described below. At least five cardiac cycles were digitized for each patient on each occasion.

Left ventricular end systolic and end diastolic cavity dimensions were measured using standard criteria from the parasternal long axis view, ${ }^{26}$ and the left ventricular ejection fraction was calculated using the cube formula. ${ }^{27,28}$ Systolic time intervals were measured from the simultaneous electrocardiograms and aortic echocardiograms ${ }^{29}$ and the ratio of the pre-ejection period to the corrected left ventricular ejection time (PEP/LVET(C)) was calculated as an additional heart-rate-independent index of left ventricular systolic function. ${ }^{30,31}$

Abnormalities of left ventricular diastolic function often precede abnormalities of systolic function ${ }^{32,33}$ and may therefore be more sensitive to small druginduced changes in myocardial performance than measures of global systolic function. Events during early diastole were characterized by measurement of isovolumic relaxation time (IVRT) and quantitative analysis of the early (E) Doppler velocity profile. Events during mid to late diastole were quantified by measurement of the diastasis period (the time interval from the termination of early inflow to the onset of late inflow) and quantitative analysis of the late (A) Doppler velocity profile. The E and A waves were quantitatively analysed by measurement of their peak velocities, acceleration and deceleration gradients, acceleration and deceleration times, the area under each peak, and the ratio of peak early to peak late inflow velocities. ${ }^{34-36}$ Measurements were made during inspiration, expiration and inspiratory apnoea.

\section{Statistical analysis}

We used the statistical package SpSS-PC. Analysis of variance was used to test for changes of the variables over time. A logarithmic transformation was used before analysis of the $\mathrm{NN}_{50}$ count, as the data was not normally distributed. Linear regression analysis was used to test the fall in the height of the T-waves on the serial ECG recordings.

\section{Results}

The 20-day course of sodium stibogluconate $(20 \mathrm{mg} / \mathrm{kg} /$ day $)$ healed the ulcers of $11 / 12$ patients. All reported muscle aches and loss of appetite during treatment, but there were no other symptoms or signs. In particular no patient developed any cardiac symptoms.

\section{Blood pressure and heart rate}

There was a fall in the systolic and diastolic blood pressure $(p<0.05)$ associated with an increase in heart rate $(p<0.05)$ at the end of the course of treatment. Blood pressure and heart rate returned to pre-treatment levels 6 weeks after treatment stopped (Tables 2 and 4).

\section{Cardiac enzymes}

There was no change in either creatine kinase or lactate dehydrogenase during treatment. A small, but statistically significant $(p<0.05)$ rise in lactate dehydrogenase was noted at both 2 and 6 weeks after the end of treatment when the soldiers had returned to full physical duties. There was no change in creatine kinase following treatment in 10 soldiers, but in two it rose to 1497 and $416 \mathrm{IU} / \mathrm{I}$, respectively, 6 weeks after treatment stopped (Table 2). The soldier who had the highest level also had an elevated waking and sleeping heart rate (95 and 90 beats $/ \mathrm{min}$ ) at that time compared to his baseline (70 and 51) 
Table 2 Blood pressure and biochemical changes

\begin{tabular}{|c|c|c|c|c|c|c|}
\hline & \multirow{2}{*}{$\begin{array}{l}\text { Pre-treatment } \\
\text { Day } 0\end{array}$} & \multicolumn{3}{|l|}{ Treatment } & \multicolumn{2}{|l|}{ Recovery } \\
\hline & & Day 7 & Day 14 & Day 20 & Day 34 & Day 62 \\
\hline \multicolumn{7}{|l|}{ Blood pressure $(\mathrm{mmHg})$} \\
\hline Systolic & $126(3.61)$ & $116(4.76)$ & $118^{*}(2.68)$ & $113^{*}(3.03)$ & $121(2.94)$ & $118(3.08)$ \\
\hline Diastolic & $84(2.02)$ & $78(2.97)$ & $81(2.68)$ & $73 *(2.45)$ & $84.7(2.92)$ & $76(2.89)$ \\
\hline Creatine kinase (IU/I) & $99(26.8)$ & $71(21.7)$ & $56(17.4)$ & $61(19.7)$ & $105(24.3)$ & $233^{*}(119.8)$ \\
\hline Lactate dehydrogenase (IU/I) & $189(10.5)$ & $174(8.8)$ & $182(10.0)$ & $188(12.8)$ & $248^{*}(21.2)$ & $208^{*}(14.9)$ \\
\hline
\end{tabular}

* Significant change $(p<0.05)$ from pre-treatment baseline.

Data are means (SEM).

and post-treatment (83 and 62) rates. His CK remained high (545 IU/I) 14 months after treatment, but at that time only $2 \%$ was CK-MB, indicating that most was derived from skeletal muscle. There was no change in serum potassium, urea or creatinine. A rise in serum aminotransferases was noted during treatment, this is well-recognized during treatment with sodium stibogluconate. ${ }^{15,16,17}$

\section{Electrocardiography}

The cardiac electrical axis and R-wave magnitude did not change during this study. There was, however, a steady and significant $(p<0.001)$ decrease in the sum of the heights of the T waves in V4-6, often progressing to T-wave inversion as treatment progressed, followed by a return to pre-treatment levels 6 weeks later (Table 3, Figure 1).

A complete series of analysable $24-h$ recordings was available for only seven patients. There was no significant increase in supraventricular or ventricular arrhythmias with treatment. Similarly, neither the 24-h standard deviation of RR intervals nor the $\mathrm{NN}_{50}$ count changed significantly during the course of this study (Table 3).

\section{Left ventricular function}

There were no significant changes in left ventricular cavity dimensions or measures of left ventricular systolic function (Table 4). Similarly, the indices of early and late left ventricular diastolic function did not change with treatment (Table 5).

\section{Discussion}

The pentavalent antimonials, sodium stibogluconate and meglumine antimoniate, first manufactured 50 years ago, remain the mainstay of treatment for

Table 3 Electrocardiographic changes

\begin{tabular}{|c|c|c|c|c|c|c|}
\hline & \multirow{2}{*}{$\begin{array}{l}\text { Pre-treatment } \\
\text { Day } 0\end{array}$} & \multicolumn{3}{|l|}{ Treatment } & \multicolumn{2}{|l|}{ Recovery } \\
\hline & & Day 7 & Day 14 & Day 20 & Day 34 & Day 62 \\
\hline ECG axis $\left({ }^{\circ}\right)$ & $59.4(7.15)$ & $61.3(5.64)$ & $61.7(5.69)$ & $61.7(5.75)$ & $60.6(6.71)$ & $58.0(6.16)$ \\
\hline $\mathrm{R}$-wave height (mm) & $21.7(1.6)$ & $22.4(1.3)$ & $22.6(0.58)$ & $21.2(1.3)$ & $20.7(2.0)$ & $20.0(1.1)$ \\
\hline $\begin{array}{c}\text { T-wave height }(\mathrm{mm}) \\
(\mathrm{V} 4+\mathrm{V} 5+\mathrm{V} 6)\end{array}$ & $18.8(2.0)$ & $14.7^{*}(2.5)$ & $10.4^{*}(1.9)$ & $7.4^{*}(2.0)$ & $15.9(0.7)$ & $18.5(2.9)$ \\
\hline \multicolumn{7}{|l|}{ Heart rate $\left(\min ^{-1}\right)$} \\
\hline (waking) & $69.3(2.2)$ & NA & NA & $77.7^{*}(2.2)$ & NA & $72.7(4.0)$ \\
\hline (sleeping) & $51.9(1.63)$ & NA & NA & $65.0 *(3.7)$ & NA & $57.6(5.5)$ \\
\hline $\begin{array}{l}\text { Total supraventricular } \\
\text { ectopic count per } 24 \mathrm{~h}\end{array}$ & $3.14(1.85)$ & NA & NA & $1.00(0.68)$ & NA & $8.14(6.36)$ \\
\hline $\begin{array}{l}\text { Total ventricular } \\
\text { ectopic count per } 24 \mathrm{~h}\end{array}$ & $5.00(4.33)$ & NA & NA & $3.67(3.08)$ & NA & $1.50(0.72)$ \\
\hline $\begin{array}{l}\text { Standard deviation } \\
\text { RR interval }(\mathrm{ms})\end{array}$ & $198(11.2)$ & NA & NA & $158(15.0)$ & NA & $196(21.1)$ \\
\hline $\begin{array}{c}\mathrm{NN}_{50} \text { counts per } \\
24 \mathrm{~h}\left(\log _{10}\right)\end{array}$ & $4.18(0.048)$ & NA & NA & $4.01(0.11)$ & NA & $4.06(0.15)$ \\
\hline
\end{tabular}

* Significant change from pre-treatment baseline.

Data are means (SEM). NA, not applicable. 


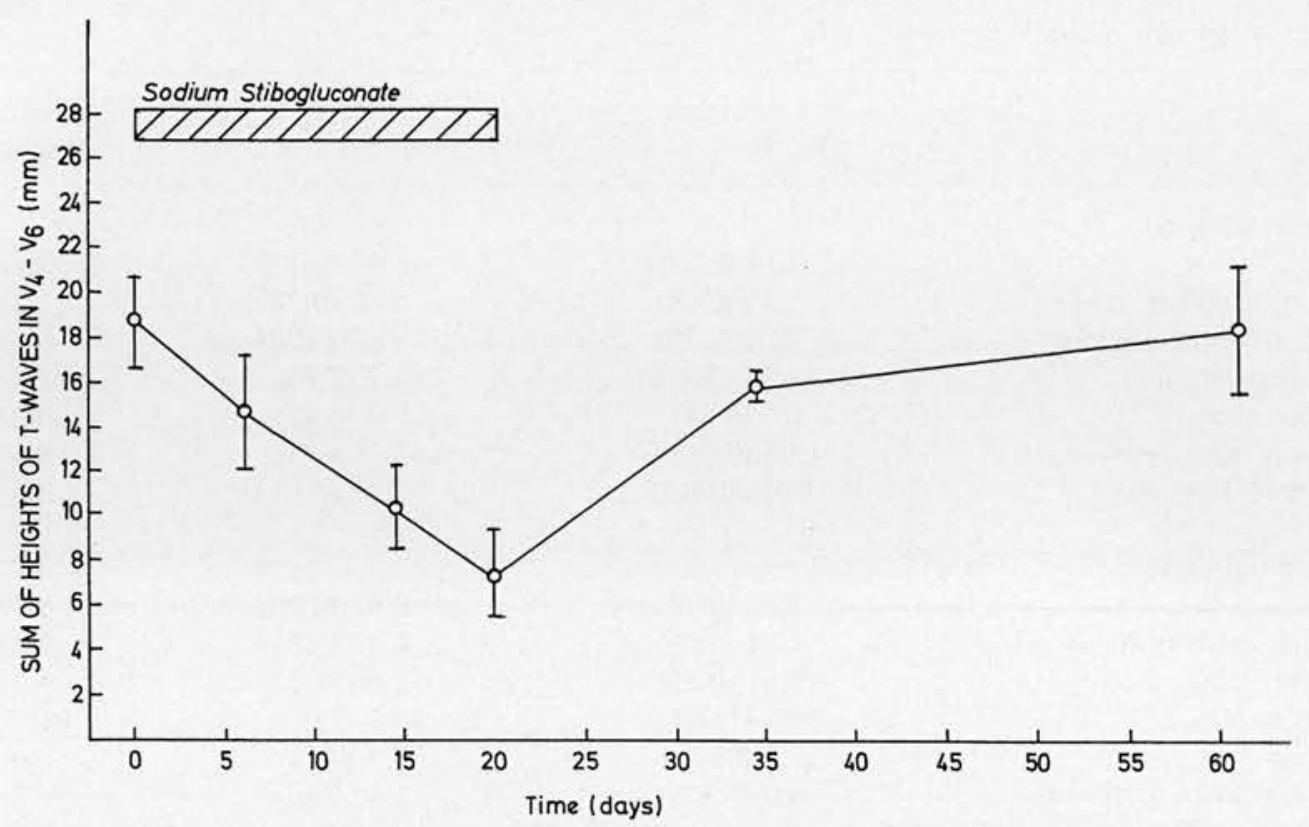

Figure 1. Sum of $T$-wave heights in leads $V 4, V 5$ and $V 6$ during treatment and recovery.

Table 4 Left ventricular systolic function

\begin{tabular}{lccc}
\hline & $\begin{array}{l}\text { Pre-treatment } \\
\text { Day 0 }\end{array}$ & $\begin{array}{c}\text { Treatment } \\
\text { Day 20 }\end{array}$ & $\begin{array}{c}\text { Recovery } \\
\text { Day } 62\end{array}$ \\
\hline LV end diastolic diameter (cm) & $5.10(0.09)$ & $5.28(0.13)$ & $5.11(0.07)$ \\
LV end systolic diameter (cm) & $3.39(0.11)$ & $3.52(0.11)$ & $3.55(0.07)$ \\
Ejection fraction (\%) & $61.5(2.5)$ & $60.2(1.6)$ & $57.6(1.8)$ \\
Duration of systole (s) & $0.37(0.006)$ & $0.37(0.011)$ & $0.39(0.0056)$ \\
LV ejection time (LVET) (s) & $0.31(0.004)$ & $0.32(0.014)$ & $0.31(0.0055)$ \\
Pre-ejection period (PEP) (s) & $0.07(0.006)$ & $0.08(0.004)$ & $0.07(0.002)$ \\
Heart rate (min $\left.{ }^{-1}\right)$ & $61.3(3.07)$ & $68.4(2.89)$ & $58.1(2.34)$ \\
(PEP/LVET) (C) $\left(\mathrm{s}^{-1}\right)$ & $0.24(0.02)$ & $0.27(0.02)$ & $0.23(0.01)$ \\
\hline
\end{tabular}

Data are means (SEM).

leishmaniasis. Their precise composition is not known and, indeed, may vary depending upon the source and batch. ${ }^{37}$ They contain moieties of very different molecular weights (range 100-4000 Da), and a variable quantity of trivalent antimony is present. $^{37,38}$ Although sodium stibogluconate is rapidly excreted, largely unchanged, by the kidney, $5 \%$ is excreted as trivalent antimony, which has a much longer half-life. ${ }^{39}$ The cardiotoxicity of trivalent antimonials is well-established. ${ }^{38}$ Our studies, ${ }^{16,18}$ and those of others, ${ }^{15}$ indicate that a 20-day course of $20 \mathrm{mg} / \mathrm{kg} /$ day, without an upper daily limit, will heal the ulcers of $90 \%$ of patients with CL. Towards the end of this course, all patients develop myalgia, and there is usually a rise in serum aminotransferases. ${ }^{16,18}$ A 20-day course has been found to represent a reasonable balance between efficacy and toxicity. In view of the likely accumulation of cardiotoxic trivalent antimony during treatment, it was important to determine whether this regimen causes cardiac damage.

The study population were all young soldiers, none were malnourished, and none had intercurrent illnesses, renal or cardiac disease. They were different from many patients who develop visceral leishmaniasis. Abnormalities detected during treatment could therefore be attributed to sodium stibogluconate, but these tests may not detect cardiotoxicity exacerbated by ischaemic or hypertensive heart disease. This study was too small to detect idiosyncratic reactions, however, cardiotoxicity associated with trivalent antimonials, and the electrocardiographic changes observed in other studies, are related to the cumulative dose of antimony administered rather than representing idiosyncratic reactions. ${ }^{12}$

The results show that this regime has no measurable effect on left ventricular systolic or diastolic function. There was no biochemical evidence of 
Table 5 Left ventricular diastolic function

\begin{tabular}{|c|c|c|c|}
\hline & $\begin{array}{l}\text { Pre-treatment } \\
\text { Day } 0\end{array}$ & $\begin{array}{l}\text { Treatment } \\
\text { Day } 20\end{array}$ & $\begin{array}{l}\text { Recovery } \\
\text { Day } 62\end{array}$ \\
\hline \multicolumn{4}{|l|}{ Mitral E-wave (apnoea) } \\
\hline Area $(\mathrm{cm})$ & $9.27(0.51)$ & $8.87(0.33)$ & $9.67(0.57)$ \\
\hline Acceleration gradient $(\mathrm{cm} / \mathrm{s})$ & $7.70(0.66)$ & $8.46(0.71)$ & $7.21(0.69)$ \\
\hline Deceleration gradient $(\mathrm{cm} / \mathrm{s})$ & $-3.76(0.29)$ & $-3.87(0.28)$ & $-3.67(0.16)$ \\
\hline Peak velocity $(\mathrm{cm} / \mathrm{s})$ & $82.5(3.57)$ & $80.1(2.70)$ & $81.5(3.59)$ \\
\hline Acceleration time $\left(\times 10^{-2} \mathrm{~s}\right)$ & $7.4(0.45)$ & $7.0(0.32)$ & $0.83(0.52)$ \\
\hline Deceleration time $\left(\times 10^{-2} \mathrm{~s}\right)$ & $15.1(0.96)$ & $15.3(0.75)$ & $15.6(0.78)$ \\
\hline Diastasis time (s) (apnoea) & $0.34(0.052)$ & $0.20(0.033)$ & $0.35(0.053)$ \\
\hline \multicolumn{4}{|l|}{ Mitral A-wave (apnoea) } \\
\hline Area $(\mathrm{cm})$ & $2.76(0.18)$ & $2.94(0.20)$ & $3.17(0.29)$ \\
\hline Acceleration gradient $(\mathrm{cm} / \mathrm{s})$ & $4.74(0.51)$ & $6.27(0.89)$ & $5.13(0.56)$ \\
\hline Deceleration gradient $(\mathrm{cm} / \mathrm{s})$ & $-3.66(0.35)$ & $-4.06(0.31)$ & $-3.33(0.22)$ \\
\hline Peak velocity $(\mathrm{cm} / \mathrm{s})$ & $39.9(1.98)$ & $44.2(3.07)$ & $41.7(2.04)$ \\
\hline Acceleration time $\left(\times 10^{-2} \mathrm{~s}\right)$ & $7.8(0.69)$ & $7.9(0.29)$ & $7.0(0.62)$ \\
\hline Deceleration time $\left(\times 10^{-2} \mathrm{~s}\right)$ & $6.0(0.42)$ & $5.4(0.38)$ & $6.2(0.41)$ \\
\hline E/A peak velocity ratio & $2.12(0.15)$ & $1.92(0.15)$ & $1.99(0.11)$ \\
\hline IVRT $\left(\times 10^{-3}\right.$ s) (apnoea) & $62(5.9)$ & $68(5.5)$ & $63(5.1)$ \\
\hline
\end{tabular}

drug-induced myocardial injury during therapy. Although a small, but statistically significant, rise in lactate dehydrogenase was noted when the soldiers returned to physical duties after treatment, this was not found when a larger sample of patients was subsequently studied. ${ }^{18}$ A significant $(p<0.05)$ increase in creatine kinase 6 weeks after the completion of therapy was also noted, but this was entirely due to a large elevation in two patients, one of whom had an elevated heart rate. Although his creatine kinase remained elevated, most was derived from skeletal muscle, which may be relevant to the myalgia reported during treatment in all patients. It was not possible to identify either of these patients when the echocardiographic recordings were examined in a blinded manner by an independent observer. The elevation in lactate dehydrogenase remains unexplained.

Treatment is associated with a significant, reversible, fall in blood pressure and an increase in heart rate. The cause of these changes is not immediately apparent from our study. Although postural hypotension was not looked for, it is likely that they represent a peripheral vasodilatory effect of the drug, since cardiac function remained normal throughout the study in all patients.

Serial electrocardiograms showed a progressive reversible fall in T-wave amplitude in the lateral chest leads during treatment, which frequently progressed to T-wave inversion. Similar changes, related to the cumulative dose of antimony administered, have been demonstrated by others, ${ }^{12}$ but our study confirms that these changes occur as treatment progresses using the currently recommended regimen. The electrocardiographic changes observed in this and other studies, ${ }^{12}$ are similar to those induced by the trivalent antimonials $s^{38}$ and therefore are probably caused by the accumulation of trivalent antimony during treatment, causing changes in ventricular repolarization. Changes in ventricular repolarization are associated with an increased risk of ventricular tachyarrhythmias, ${ }^{40}$ which may be the principle mechanism of sudden death during antimony therapy. Impaired autonomic function ${ }^{41}$ and the presence of frequent arrhythmias also increase the risk of sudden death, ${ }^{42}$ but neither of these risk factors were present during treatment with sodium stibogluconate in this study. The isolated changes in ventricular repolarization demonstrated may not significantly increase the risk of sudden death, although a larger study would be necessary to investigate this relationship in detail.

In conclusion, the standard treatment regimen for American cutaneous leishmaniasis (sodium stibogluconate $20 \mathrm{mg} / \mathrm{kg} /$ day for 20 days) is not associated with measurable impairment of cardiac function using echocardiographic parameters of systolic and diastolic function in fit young men. The electrocardiographic changes observed during treatment may be caused by the accumulation of trivalent antimony, but they were not associated with electrical instability assessed by arrhythmia analysis and heart-rate variability. However, patients with pre-existing heart disease may be more susceptible to any cardiotoxic effects of sodium stibogluconate and we cannot 
exclude more subtle abnormalities or idiosyncratic reactions.

\section{Acknowledgements}

We are grateful to Colonel D. Sim-Davis L/RAMC for referring these patients for treatment, and to $\mathrm{Dr}$ D.A. Evans for culture and isoenzyme analysis. This study was conducted whilst Major Hepburn was on a secondment from the Royal Army Medical Corps.

\section{References}

1. WHO Expert Committee. Control of the Leishmaniases. Technical Report Series 793. Geneva, World Health Organisation, 1990.

2. Bromberger-Barnea B, Stephens NL. Effects of antimony on myocardial performance in isolated and intact canine hearts. Am Ind Hyg Assoc J 1965; 26:404-8.

3. Honey $M$. The effects of sodium antimony tartrate on myocardium. Br Heart J 1961; 22:601-16.

4. Davis $A$. The effects of antimony dimercaptosuccinate on the electrocardiogram. Br Heart J 1961; 23:291-6.

5. Sommers K, Rosanelli JD. Electrocardiographic effects of antimony dimercaptosuccinate (Astiban). Br Heart J 1962; 24:187-91.

6. Surwicz B. Effects of drugs on the electrocardiogram. Prog Cardiovasc Dis 1970; 13:26-55.

7. Sapire DW, Silverman NH. Myocardial involvement in antimonial therapy; a case report of acute antimony poisoning with serial ECG changes. S Afr Med J 1979; 44:948-50.

8. Stemmer KL. Pharmacology and toxicology of heavy metals: antimony. Pharmac Ther A 1976; 1:157-60.

9. Anonymous. Antimony and the Heart. Br Med J 1961; 1:1665-6.

10. Dempsey J. Leishmaniasis in the Sudan Republic xxvi. Electrocardiographic findings in Sudanese Kala-azar. East Afr Med J 1965; 42:131-4.

11. Kaplan RJ, Wilkin JL, Hartman DL. Treatment of Leishmaniasis with Sodium antimony gluconate: transitory ECG changes. South Med J 1978; 71:469-70.

12. Chulay JD, Spencer HC, Mugambi M. Electrocardiographic changes during treatment of leishmaniasis with pentavalent antimony (sodium stibogluconate). Am J Trop Med Hyg 1985; 34:702-9.

13. Henderson A, Jolliffe DS. Cardiac effects of sodium stibogluconate. Br J Clin Pharmacol 1985; 19:73-7.

14. Antezana G, Zaballos R, Mendoza C, Lyevre P, Cardenas F, Noriega I, Ugarte H, Dedet JP. Electrocardiographic alterations during treatment of mucocutaneous leishmaniasis with meglumine antimoniate and allopurinol. Trans R Soc Trop Med Hyg 1992; 86:31-3.

15. Ballou WR, McClain JB, Gordo DM, Shanks GD, Andujar J, Berman JD, Chulay JD. Safety and efficacy of high-dose sodium stibogluconate therapy of American cutaneous leishmaniasis. Lancet 1987; ii:13-16.

16. Hepburn NC, Tidman MJ, Hunter JAA. Cutaneous leishmaniasis in British Troops from Belize. Br J Dermatol 1993; 128:63-8.
17. Herwaldt BL, Berman JD. Recommendations for treating leishmaniasis with sodium stibogluconate (Pentostam) and review of pertinent clinical studies. Am J Trop Med Hyg 1992; 46:296-306.

18. Hepburn NC, Tidman MJ, Hunter JAA. Aminosidine versus sodium stibogluconate therapy for cutaneous leishmaniasis from Belize. Trans R Soc Trop Med Hyg (in press).

19. Fisch $C$. Electrocardiography and vectorcardiography. In: Braunwald $\mathrm{E}$, ed. Heart disease. A textbook of cardiovascular medicine. Philadelphia, Saunders, 1992:116-60.

20. Neilson JMM. Computer detection of ventricular ectopic beats: On line and Off. In: Computers in cardiology. Los Alamitos, IEEE Computer Press, 1975:33-5.

21. Nolan J, Flapan AD, Capewell S, MacDonald TM, Neilson JMM, Ewing DJ. Decreased cardiac parasympathetic activity in chronic heart failure and its relation to left ventricular function. Br Heart J 1992; 67:482-5.

22. Verrier RL, Lown B. Sympathetic-parasympathetic interactions and ventricular electrical stability. In: Schwartz PJ, Brown AM, Malliani A, Zanchetti A, eds. Neural mechanisms in cardiac arrhythmias. New York, Raven Press, 1975:75-86.

23. Murray A, Ewing DJ, Campbell IW, Neilson JMM, Clarke BF. RR interval variations in young male diabetics. $\mathrm{Br}$ Heart J 1975; 37:882-5.

24. Ewing DJ. Analysis of heart rate variability. In: Bannister R, Mathias CJ, eds. Autonomic failure. A textbook of clinical disorders of the autonomic nervous system. Oxford, Oxford University Press, 1992:312-33.

25. Ewing DJ, Neilson JMM, Travis P. New method for assessing cardiac parasympathetic activity using 24 hour electrocardiograms. Br Heart J 1984; 53:396-402.

26. Sahn DJ, DeMaria A, Kisslo J, Weyman A. Recommendations regarding quantification in $\mathrm{M}$-mode echocardiography: Results of a survey of echocardiographic measurements. Circulation 1978; 58:1072-83.

27. Mason SJ, Fortuin NJ. The use of echocardiography for quantitative evaluation of left ventricular function. Prog Cardiovasc Dis 1978; 21:1072-83.

28. Freigenbaum H. Echocardiography (4th edn). Philadelphia, Lea and Frebiger, 1986.

29. Hirchfeld S, Meyer R, Schwartz DC. Measurement of right and left ventricular systolic time intervals by echocardiography. Circulation 1975; 51:304-9.

30. Benchimol A, Matsup S. Ejection time before and after aortic valve replacement. Am J Cardiol 1971; 27:244-9.

31. Gutgesell HP, Paquet M, Duff DF, McNamara DG. Evaluation of left ventricular size and function by echocardiography. Circulation 1977; 56:457-62.

32. Bonow RO, Bacharach SL, Green MU, Kent KM, Rosing DR, Lipson LC, Leon MB, Epstein SE. Impaired left ventricular diastolic filling in patients with coronary artery disease: assessment with radionuclide angiography. Circulation 1981; 64:315-23.

33. Bonow RO, Rosing DR, Bacharach SL, Green MV, Kent KM, Lipson LC, Maron BJ, Leon MB, Epstein SE. Effects of verapamil on left ventricular systolic function and diastolic filling in patients with hypertrophic cardiomyopathy. Circulation 1981; 64:787-96.

34. Nishimura RA, Abel MD, Hatle LK, Tajik AJ. Assessment of diastolic function of the heart; background and current applications of Doppler echocardiography. Part II: clinical studies. Mayo Clin Proc 1989; 64:181-204. 
35. Labovitz AJ, Pearson AC. Evaluation of left ventricular diastolic function: Clinical relevance and recent Doppler echocardiographic insights. Am Heart J 1987; 114:836-61.

36. Spirito $P$, Maron BJ. Doppler echocardiography for assessing left ventricular diastolic function. Ann Intern Med 1988; 109:122-6.

37. Marsden PD. Pentavalent antimonials: Old Drugs for New Diseases. Rev Soc Bras Med Trop 1985; 18:187-98.

38. Berman JD. Chemotherapy for Leishmaniasis: Biochemical Mechanisms, Clinical Efficacy and Future Strategies. Rev Infect Dis 1988; 10:560-86.

39. Bryceson A. Therapy in Man. In: Peters W, Killick-Kendrick
R, eds. The Leishmaniases in Biology and Medicine, vol. 2. London, Academic Press, 1987:847-907.

40. Merx W, Yoon MS, Han J. The role of local disparity in conduction and recovery time on ventricular vulnerability to fibrillation. Am Heart J 1977; 94:603-10.

41. Kleiger RE, Miller JP, Bigger JT, Moss AJ. Decreased heart rate variability and its association with increased mortality after acute myocardial infarction. Am J Cardiol 1987; 59:256-62.

42. Multicentre Post Infarction Research Group. Risk stratification and survival after myocardial infarction. N Engl J Med 1983; 309:331-6. 